\title{
Organisationskonfigurationen und interne Arbeitsmarkte : eine theoretisch-empirische Untersuchung von Arbeitsorganisationen im Zeichen interner Arbeîtsmarkte
}

Citation for published version (APA):

Schwan, R. (1993). Organisationskonfigurationen und interne Arbeitsmarkte : eine theoretisch-empirische Untersuchung von Arbeitsorganisationen im Zeichen interner Arbeitsmarkte. [Doctoral Thesis, Maastricht University]. Datawyse / Universitaire Pers Maastricht. https://doi.org/10.26481/dis.19930609rs

Document status and date:

Published: 01/01/1993

DOI:

10.26481/dis.19930609rs

Document Version:

Publisher's PDF, also known as Version of record

Please check the document version of this publication:

- A submitted manuscript is the version of the article upon submission and before peer-review. There can be important differences between the submitted version and the official published version of record. People interested in the research are advised to contact the author for the final version of the publication, or visit the DOI to the publisher's website.

- The final author version and the galley proof are versions of the publication after peer review.

- The final published version features the final layout of the paper including the volume, issue and page numbers.

Link to publication

\footnotetext{
General rights rights.

- You may freely distribute the URL identifying the publication in the public portal. please follow below link for the End User Agreement:

www.umlib.nl/taverne-license

Take down policy

If you believe that this document breaches copyright please contact us at:

repository@maastrichtuniversity.nl

providing details and we will investigate your claim.
}

Copyright and moral rights for the publications made accessible in the public portal are retained by the authors and/or other copyright owners and it is a condition of accessing publications that users recognise and abide by the legal requirements associated with these

- Users may download and print one copy of any publication from the public portal for the purpose of private study or research.

- You may not further distribute the material or use it for any profit-making activity or commercial gain

If the publication is distributed under the terms of Article $25 \mathrm{fa}$ of the Dutch Copyright Act, indicated by the "Taverne" license above, 
Organisationskonfigurationen und interne Arbeitsmärkte 
()1993 Rolf Schwan, Maastricht

CIP-GEGEVENS KONINKLUKE BIBLIOTHEEK, DEN HAAG

Schwan, Rolf

Organisationskonfigurationen und interne arbeitsmärkte : eine theoretisch-empirische untersuchung von arbeitsorganisationen im Zeichen interner Arbeitsmärkte / Rolf Schwan. - Maastricht : Universitaire Pers Maastricht. - III.

Thesis Maastricht. - With ref. - With summary in Dutch and English.

ISBN 90-5278-079-x

NUGI $684 / 685$

Subject headings: internal labour market / organization theory.

Boekverzorging: Datawyse I Universitaire Pers Maastricht 


\title{
Organisationskonfigurationen und interne Arbeitsmärkte
}

\author{
Eine theoretisch-empirische Untersuchung \\ von Arbeitsorganisationen \\ im Zeichen interner Arbeitsmärkte
}

\section{PROEFSCHRIFT}

ter verkrijging van de graad van doctor

aan de Rijksuniversiteit Limburg te Maastricht, op gezag van de Rector Magnificus, Prof. mr. M. J. Cohen,

volgens het besluit van het College van Dekanen,

in het openbaar te verdedigen

op woensdag, 9 juni 1993 om 16.00 uur

\section{DISSERTATION}

zur Erlangung des Doktorgrades

an der Rijksuniversiteit Limburg zu Maastricht, auf Anordnung des Rector Magnificus, Prof. mr. M. J. Cohen, gemäß dem Beschluß des College van Dekanen,

öffentlich zu verteidigen am

Mittwoch, den 9. Juni 1993 um 16.00 Uhr

door/durch

Rolf Schwan

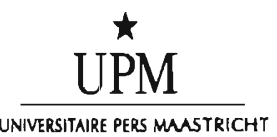


PROMOTOR:

Prof. dr. H. Schreuder

CO-PROMOTOR:

Dr. J. Soeters

BEOORDELINGSCOMMISSIE:

BEURTEILUNGSKOMMISSION:

Prof. dr. A. Sorge (voorzitter/Vorsitzender)

Rijksuniversiteit Limburg

Humboldt-Universität zu Berlin

Dr. P. de Gijsel

Rijksuniversiteit Limburg

Dr. A. de Grip

Rijksuniversiteit Limburg

Prof. dr. R. Ziegler

Ludwig-Maximilians-Universität München 
In our present situation, the task is not, as is sometimes assumed, the relatively easy one of filling 'emply boxes' of theory with a content of empirical knowledge about reality. For our theoretical

boxes are empty primarily because they are not built in such a way that they can hold reality.

Gunnar Myrdal

gewidmet meiner Familie 
<smiles>[CH]</smiles> 


\section{Vorwort}

Diese Arbeit erforderte eine Organisationsform, die bislang in der einschlägigen Literarur nur am Rande erwähnt wird, jedoch von der Bedeutung zunimmt. Möglicherweise könnte dies die 'siebte' Organisationform sein neben den von Mintzberg behandelten sechs Organisationskonfigurationen. Es handelt sich um die Netzwerkorganisation. Die Netzwerkorganisation zeichnet sich durch eine Vielzahl von partizipierenden Personen aus, die am Zustandekommen und Ausführung des Projektes ihren Anteil haben. An das Projekt waren vom Beginn an hohe Erwartungen gekoppelt. Der Zielvorstellung der integrativen Sichtweise von Betriebs- und Volkswirtschaft am Beispiel des internen Arbeitsmarktes waren Grenzen gesetzt.

Das dynamische Netzwerk in seiner lnnovationsorientierung bezog sich auf die folgenden Personen. Mein besonderer Dank gilt Hein Schreuder, dessen Ruf ins Land der Regenten, Rebellen und Reformatoren ich gefolgt bin. Er eröffnete mir die Chance einer zweiten Primärsozialisation mit der entsprechenden mentalen Programmierung. Mein besonderer Dank gilt Sjo Soeters für die vielfältige Unterstützung sowie Begleitung in jeder Beziehung, ferner den Mitgliedem der Beurteilungskommission.

Teil der Basisnetzwerkstruktur bildete die Abteilung mit allen Mitarbeitem, denen ich Dank schulde, insbesondere Bas van Diepen, Ellen Dittrich, Geert Hofstede, Ad van Iterson, Bas Koene, Jos Lasker-Vrijens, Maria Mulder, Jan Nijhuis, Rene Olie, Sjoerd Romme, Arndt Sorge, Arjen van Witteloostuijn und Mieke van Zutphen sowie als Studentassistenten Nicole Classen, Anne-Wil Harzing und Woody van Olffen.

Der volkswirtschaftliche 'Flügel' des Netzwerkes wird angeführt durch Guido Wolfs, dem ich zahlreiche Anregungen und 'Auseinandersetzungen' in der Integrationsphase verdanke. Besonderer Dank gilt Peter de Gijsel als einem der Projekt 'founding fathers' sowie Joan Muysken und Jürgen Backhaus, Hans Heijke und Andries de Grip.

Hinsichtlich der Datenverarbeitung bin ich dankbar für den Rat von Ellen Dittrich, Wim Gijselaers, Carin Hendrikx, Paul Hick, Hub van Kan, Bas Koene, Jos Lemmink, Frans Nijhuis, Sybrand Schim van der Loeff und Harry Wetzelaer.

Bei der Bewältigung des täglichen Netzwerkmanagements konnte ich auf die Kompetenz von Marcel Lever zurückgreifen. Ron Dekker sorgte als Krisenberater dafür, daß der Arbeitsmarkt- und Organisationsaspekt im ausgewogenen Verhältnis standen. Begleitung erfuhr ich ebenfalls durch die RL-Personalabteilung besonders durch May Berns.

Für eine optimale Informationslogistik danke ich den Mitarbeitern der Universitätsbibliothek. Von den Entzugserscheinungen des deutschen Sprachgebrauchs befreiten mich Ruth 
und Robert Stöcker. Charles Pahud de Mortanges gab mir das Gefühl eines 'native speakers'.

Den extemen Netzwerkteil bildeten die vier untersuchten Organisationen, ohne deren Mitwirken die Untersuchung nicht zustande gekommen wäre. Der jeweiligen Organisationsleitung sowie den beteiligten Mitarbeitem gilt mein Dank.

Feed back durch Beurteilung von Manuskriptteilen erhielt ich von Sten Jönsson (stellvertretend für drei anonyme Reviewer), Ray Loveridge, Michael Poole sowie Christian Scholz.

Netzwerkrelevant war die Begegnung mit Peter Doeringer und Werner Sengenberger, Henry Mintzberg sowie John T. Addison.

Ich habe die Netzwerkstruktur gestalten können. Thr wird ein hoher Zukunftswert nachgesagt. Einen Teil davon konnte ich in der Gegenwart erfahren.

Maastricht 1993

Rolf Schwan 


\section{Inhaltsverzeichnis}

1 Einleitung: Der Arbeitsmarkt zwischen Problemlagen und Anpassung 1

1.1 Der Beitrag der Arbeitsmarktforschung zur Lösung der Beschäftigungsprobleme 2

1.2 Der 'Markt' für Arbeit als Anpassungsprozeß 4

1.3 Begriff und Funktionsweise von Arbeitsmärkten 6

1.4 Zentrale Fragestellung 7

1.5 Weiterer Aufbau der Studie 9

2 Arbeitsmarktstrukturdimensionen in Theorien interner Arbeitsmärkte 12

2.1 Interne Arbeitsmärkte aus institutionalistischer Arbeitsmarkttheorieperspektive 13

2.2 Die Funktionsweise von intemen Árbeitsmärkten 16

$\begin{array}{ll}2.3 \text { Schlußfolgerungen } & 29\end{array}$

3 Organisationstheoretische Erklärung interner Arbeitsmärkte 30

3.1 Ausgangspunkte einer organisationstheoretischen Erklärung interner
Arbeitsmärkte

3.2 Kennzeichen der Organisation des internen Arbeitsmarktes 33

3.3 Interne Arbeitsmärkte im Lichte der neuen institutionellen Ökonomie 37

3.4 Inteme Arbeitsmärkte im Lichte der Kontingenztheorie 45

3.5 Integration der Arbeitsorganisationsperspektive 47

$\begin{array}{ll}3.6 & \text { Die Allokation auf internen Arbeitsmärkten } \\ 3.7 & 63\end{array}$

3.7 Konzeptionalisierung der Mobilität auf dem intemen Arbeitsmarkt 63

$\begin{array}{ll}3.8 \text { Konzeptionalisierung der Besetzung offener Stellen } & 68\end{array}$

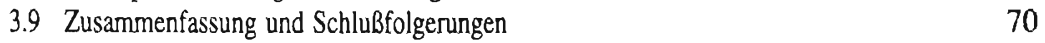

4 Zentrale Fragestellung und erkenntnisleitende Hypothesen der Untersuchung

4.1 Erkenntnisleitende Fragestellung $\quad 71$

$\begin{array}{ll}4.2 \text { Hypothesenformulierung } & 72\end{array}$

5 Methoden der empirischen Untersuchung $\quad 77$

$\begin{array}{ll}5.1 \text { Kriterien der Auswahl der untersuchten Organisationen } & 77\end{array}$

5.2 Die qualitative und quantitative Untersuchung 80

$\begin{array}{ll}5.3 \text { Methodische Verantwortung der Untersuchung } & 86\end{array}$

6 Die empirisch untersuchten Organisationen 88

$\begin{array}{ll}6.1 \text { Das Krankenhaus } & 88\end{array}$

6.2 Die Sozialversicherung 99

$\begin{array}{ll}\text { 6.3 Die Wirtschaftsprüfungsgesellschaft } & 107\end{array}$

6.4 Das Produktionsuntemehmen 112 
6.5 Organisationsvergleich von Arbeitsmarktcharakteristiken

6.6 Organisationskennzeichen zur Bestimmung von Personalpolitik im Rahmen der sozialen Untemehmensführung

6.7 Zusammenfassende Bewertung

7 Empirische Befunde der Infrastrukturanalyse interner Arbeitsmärkte

7.1 Beschreibung der Variablen der Mobilitätsanalyse

7.2 Werteübersicht der Variablen der Mobilitätsanalyse

7.3 Erweiterte statistische Analysen

7.4 Analyse der Beschäftigungsdauer von Arbeimehmern in den verschiedenen Organisationen

7.5 Schlußfolgerungen

8 Die offene Stellenanalyse

8.1 Interaktion zwischen ex- und intemer Stellenbesetzung 160

$\begin{array}{ll}\text { 8.2 Die inteme Besetzung freiwerdender Stellen } & 172\end{array}$

8.3 In- bzw. externe Stellenbesetzung nach Parts 177

$\begin{array}{lll}8.4 & \text { Schlußfolgerungen } & 178\end{array}$

9 Zusammenfassung und Schlußfolgerungen aus der Untersuchung 181

9.1 Zusammenfassung der wichtigsten Untersuchungsergebnisse 181

9.2 Segmentierung des betrieblichen Arbeitsmarktes 189

$\begin{array}{ll}9.3 \text { Rïckschlüsse auf die Beschäftigungsstrategie } & 189\end{array}$

$\begin{array}{lll}9.4 & 191\end{array}$

9.5 Weiterführende Untersuchungsperspektiven 193

9.6 Inteme Arbeitsmärkte im gesellschaftlichen Kontext 194

Samenvatting / Zusammenfassung in niederländischer Sprache 197

Summary / Zusammenfassung in englischer Sprache 199

$\begin{array}{ll}\text { Anlage } & 201\end{array}$

$\begin{array}{ll}\text { Literatur } & 208\end{array}$

$\begin{array}{ll}\text { Curriculum Vitac } & 223\end{array}$ 


\section{Tabellenverzeichnis}

Tab. 1: Allgemeine Kennzeichen der Organisationskonfigurationen

Tab. 2: Assoziation von Organisationskonfiguration mit Rekrutierungsstrategie (ideaitypisch)

Tab. 3: Infrastrukturkonzept intemer Arbeitsmärkte für Maschinen- und professioneller Bürokratie

Tab. 4: Stichprobe und Stellenbesetzung zum Erhebungsjahr 1989

Tab. 5: Verteilung der offenen Stellen auf die Organisationen zum Erhebungsjahr 1989

Tab. 6: Lebensaltersverteilung im Zeitraum 1984, 1986, 1988 in vier Organisationen in Prozenten

Tab. 7: Verteilung Männer/Frauen im Jahre $84,86,88$ in Prozenten

Tab. 8: Durchschnittliche Tätigkeitsdauer von Arbeitnehmern (für die Jahre 84, 86, 88) in Prozenten

Tab. 9: Organisationsaustritt nach Gründen in den Jahren $84,86,88$ in Prozenten

Tab. 10: Kemaktivitäten im Personalbereich in Prozenten
Tab. 11: Vereilung der Zahl der Befórderungen auf die untersuchten Organisationen

Tab. 12: Durchschnittliche Gehaltszuwachsentwicklung nach Organisation

Tab. 13: Verteilung der Funktionen zum Erhebungszeitpunkt (1989) über Organisationen und Funktionsniveaus im Rahmen der Stichprobe

Tab. 14: Verteilung der Formen der Weiterbildung

Tab. 15: Schulausbildungsniveau der im Rahmen der Stichprobe erfaßten Arbeitnehmer

Tab. 16: Verteilung der Fälle von Part-Mobilität

Tab. 17: Variablenbeschreibung: Infrastrukturanalyse 137

Tab. 18: Ergebnisse der Varianzanalyse Infrastruktur 138

Tab. 19: Signifikante Pearsonkorrelationen der Infrastrukturanalyse (Anlage) 201

Tab. 20: Signifikante Pearsonkorrelationen der Mobilitätsanalyse (professionelle Bürokratien) (Anlage)

Tab. 21: Signifikante Pearsonkorrelationen der Mobilitätsanalyse (Maschinenbürokratien) (Anlage)

Tab. 22: Beförderungsanalyse: total

Tab. 23: Beförderungsanalyse: total; Diskriminantenanalyse (Anlage)

Tab. 25: Beförderungsanalyse: Maschinenbürokratien Diskriminantenanalyse (Anlage)

Tab. 26: Gehaltszuwachsanalyse: total

Tab. 27: Gehaltszuwachsanalyse: prof. Bürokratie 
Tab. 28: Gehaltszuwachsanalyse: Maschinenbürokratie

Tab. 29: Breakdown der Variablen: 'Jahre in Dienst' (JID) und

'Beschäftigungsdauer'. (TIJD)

Tab. 30: Funktionswechsel in der Langzeitbetrachtung

Tab. 32: Ergebnisse der Varianzanalyse der Betriebszugehörigkeit und Funktionswechsel

Tab. 33: Breakdown der Variablen: 'Jahre in Dienst' (JD) und in Bezug auf 'parts' (Anlage)

Tab. 34: Beschältigungsdaueranalyse: Totalanalyse

Tab. 35: Beschäftigungsdaueranalyse: Professionelle Bürokratien

Tab. 36: Beschäftigungsdaueranalyse: Maschinenbürokratien

Tab. 37: Offene Stellenbesetzung in 4 Organisationen im Dreijahreszeitraum 1986-1988

Tab. 38: Inteme Stellenbesetzung: Bedeutung der abhängigen Variable (DIP)

Tab. 39: Funktionsverteilung der zu besetzenden offenen Stellen im Dreijahreszeitraum

Tab. 40: Verteilung der Entstehungsgründe offener Stellen und deren Besetzung im Dreijahreszeitraum

Tab. 41: Werteübersicht der offenen Stellenanalyse

Tab. 42: Werteübersicht der internen Stellenbesetzung 167

Tab. 43: Varianzanalyse offene Stellen

Tab. 44: Varianzanalyse der Stellenbesetzung

Tab. 45: Signifikante Pearsonkorrelationen der Vakanzentotalanalyse (Anlage) 204

Tab. 46: Signifikante Pearsonkorrelationen der Vakanzenteilanalyse (Professionelle Bürokratien) (Anlage)

Tab. 47: Signifikante Pearsonkorrelationen der Vakanzenteilanalyse (Maschinenbürokratien) (Anlage)

Tab. 48: Besetzung offener Stellen: total; Pearsonkorrelation; Regressionsanalyse 169

Tab. 49: Besetzung offener Stellen: total; Diskriminantenanalyse (Anlage)

Tab. 50: Besetzung offener Stellen: prof. Bürokratien; Pearsonkorrelation; Regressionsanalyse

Tab. 51: Besetzung offener Stellen: professionelle Bürokratien; Diskriminantenanalyse (Anlage)

Tab. 52: Besetzung offener Stellen: Maschinenbürokratien; Pearsonkorrelation; Regressionsanalyse

Tab. 53: Besetzung offener Stellen: Maschinenbürokratien; Diskriminantenanalyse (Anlage)

Tab. 54: Zusammenfassende Ergebnisübersicht der offenen Stellenanalyse

Tab. 55: Signifikante Pearsonkorrelationen der intemen Besetzungsanalyse offener Stellen

Tab. 56: Signifikante Pearsonkorrelationen der intemen Besetzungsanalyse offener Stellen in professionellen Bürokratien

Tab. 57: Signifikante Pearsonkorrelationen der internen Besetzungsanalyse offener Stellen in Maschinenbürokratien 
Tab. 58: Interne Besetzung offener Stellen: total; Pearsonkorrelation; Regressionsanalyse

Tab. 59: Besetzung offener Stellen: prof. Bürokratien; Pearsonkorrelation; Regressionsanalyse

Tab. 60: Besetzung offener Stellen: Maschinenbürokratien; Pearsonkorrelation; Regressionsanalyse

Tab. 61: Zusammenfassende Ergebnisübersicht der offenen Stellenanalyse bei interner Stellenbesetzung

Tab. 62: Offene Stellenbesetzung auf dem organisationsintemen Arbeitsmarkt im Dreijahreszeitraum nach Parts 


\section{Abbildungsverzeichnis}

Abb. 1: Hypothetische Position der Organisationen im Spektrum Hierarchie/Markt 9

Abb. 2: Analytische Strukturen intemer Arbeitsmärkte nach Doeringer/Piore 19

Abb. 3: Analytische Arbeitsmarktstrukturen im Vergleich 28

Abb. 4: Intrinsische Kennzeichen und extrinsische Determinanten der Organisation interner Arbeitsmärkte 34

Abb. 5: Das 'organizational failure framework' 39

Abb. 6: Unternehmens-'governance structures' 41

Abb. 7: Arbeitsorganisationsperspektive von Williamson und Mintzberg 48

Abb. 8: Synopse vergleichbarer Arbeitsorganisationstypen nach Autoren $\quad 50$

Abb. 9: Rekrutierungsstrategietypen 56

Abb. 10: Formen von Allokationsgrenzen nach Mintzberg, DiPrete und anderen 62

Abb. 11: Die in die empirische Untersuchung eingegangenen Organisationen 78

Abb. 12: Idealtypische Organisation mit Andeutung der Organisationsteile (parts) nach Mintzberg

Abb. 13: Organigramm des Krankenhauses

Abb. 14: Besetzung der Organisationsteile (parts) des Krankenhauses mit Beschäftigten

Abb. 15: Funktionsketten im Krankenhaus

Abb. 16: Organigramm der Sozialversicherung 101

Abb. 17: Besetzung der Organisationsteile (parts) der Sozialversicherung mit Beschäftigten

Abb. 18: Funktionsketten in der Sozialversicherung 106

Abb. 19: Organigramm der Wirtschaftsprüfungsgesellschaft 108

Abb. 20: Besetzung der Organisationsteile (parts) der Wirtschaftsprüfungsgesellschaft mit Beschäftigten $\quad 109$

Abb. 21: Funktionskette in der Wirtschaftsprüfungsgesellschaft 112

Abb. 22: Organigramm des Produktionsuniemehmen 114

Abb. 23: Besetzung der Organisationsteile (parts) des Produktionsunternehmens mit Beschäftigten

$\begin{array}{ll}\text { Abb. 24: Funktionsketten im Produktionsuntemehmen } & 120 \\ \text { Abb. 25: Mobilität zwischen Parts } & 134\end{array}$

Abb. 26: Struktur des Datensatzes der Variable (FUWI) im Rahmen der Ereignisanalyse 
"Der interne Arbeitsmarkt bietet ... ein klassisches Beispiel für die Entgegensetzung von 'offenem' Marktaustausch und 'geschlossener' interner Regulierung."

(Windolf/Hohn, 1984, S. 12/13)

\section{Einleitung: der Arbeitsmarkt zwischen Problemlagen und Anpassung}

Dieses Einführungskapitel zeigt in groben Zügen einige der Beschäftigungsprobleme auf, mit denen sich Wirtschaft, Staat und Gesellschaft konfrontiert sehen. Ausgehend von einer kurzen Einschätzung der Beschäftigungsentwicklung und den Antworten, die die Wissenschaft bietet, wird das Forschungsfeld herausgearbeitet, auf das sich diese Untersuchung bezieht.

Schließlich erfolgt in diesem Kapitel eine begriffliche Orientierung in Bezug auf den Arbeitsmarkt allgemein sowie in- und extemem Arbeitsmarkt im besonderen. Vorgestellt wird die zentrale Fragestellung der Untersuchung sowie deren theoretische Fundamente. Eine Übersicht zu den weiteren Inhalten der Studie sowie zur Bedeutung der empirischen Untersuchung schließt sich an.

Die Arbeitslosenquoten bieten nur einen Anhaltspunkt für die qualitative Veränderung in der Beschäftigungsentwicklung. Die Zahl der Personen, die nicht wieder einer Beschäftigung zugeführt werden können, nimmt zu. Dabei handelt es sich nicht allein um "benachteiligte Gruppen von Arbeitnehmem" (Offe/Hinrichs, 1977), sondem es kommen weitere Gruppen (z. B. Akademiker) hinzu. Windolf/Hohn (1984) greifen in diesem Zusammenhang den von Max Weber geprägten Begriff der "sozialen SchlieBung" auf, der die Tendenz zur institutionellen Verfestigung in kapitalistisch organisierten Gesellschaften kennzeichnet. Damit wir die Erscheinung angesprochen, daß soziale Gruppen mit bestimmten Kennzeichen von Erwerbschancen ausgeschlossen werden (Edwards/Reich/ Gordon, 1975). Die Vorstellung von der 'Offenheit des Marktes' erfährt auf diese Weise eine Verkehrung ins Gegenteil. Auch interne Arbeitsmärkte tragen zu dieser Entwicklung bei. 


\subsection{Der Beitrag der Arbeitsmarktforschung zur Lösung der Beschäftigungsprobleme}

Mit den Beschäftigungsproblemen gingen Bemühungen einher, mehr wissenschaftlichen Aufschluß über ein komplexes Gebiet zu gewinnen. In den letzten Jahren erfahren arbeitsmarktbezogene Forschungsaktivitäten sowohl quantitativ als auch qualitativ besondere Aufmerksamkeit (Sadowski, 1990). Sie bilden einen Schwerpunkt innerhalb der Wirtschafts- und Sozialwissenschaften. Die Arbeitsmarkt- und Berufsforschung als Teilgebiet hat in den letzten Jahren eine weitere Institutionalisierung und Etablierung erfahren. Daneben befruchten benachbarte Disziplinen das Forschungsfeld. Die Forschungslandschaft ist in den verschiedenen Länder unterschiedlich ausgeprägt. Trendsetter seit dem Ende der sechziger Jahre sind Untersuchungen aus den Vereinigten Staaten (Averitt, 1968; Edwards/Reich/Gordon, 1975), die durch ihre Arbeiten das Entstehen einer eigenen Forschungskultur in Europa beeinflußt haben. Gegenstand war die wirtschaftliche Strukturveränderung (duale Ökonomie) und die Problematisierung der Folgen für den Arbeitsmarkt. Ein weiterer Forschungsschwerpunkt lag in der Antidiskriminienungsforschung, die nicht nur auf die verschiedenen Gruppen von Minderheiten am Arbeitsmarkt zielte, sondem auch auf geschlechtsspezifische Diskriminierung (Arrow, 1973).

In Europa sind in dieser Zeit die ersten Aktivitäten zur Institutionalisierung einer Arbeitsmarkt- und Berufsforschung erfolgt. Bis heute beeinflussen die Impulse der amerikanischen Arbeitsmarktforschung die europäische Arbeitsmarktforschungskultur (Brüderl, 1991). Diese ist institutionalisiert und hat einen Qualitätsstandard erreicht, der der amerikanischen in nichts nachsteht (Sengenberger, 1978; Sadowski, 1990).

Als Maßstab für ein interdisziplinäres Zusammenwirken mehrerer Fachdiszipline in dieser Arbeit soll hier für die Arbeitsmarktsicht der Begniff "labor economics" benutzt werden, da dieser einen Begriff von Arbeit beinhaltet, der über das traditionelle Denkmuster von Arbeit als Mittel der Produktion (Bolle, 1976) hinausgeht. McConnel/Brue (1988, S. 1) definieren ihn wie folgt:

"Labor economics is the field of economics which examines the organization, functioning, and outcomes of labor markets; the decisions of prospective and present labor market participants and the public policies which relate to the employment and payment of labor resources."

Mit dieser Definition werden auch die wichtigsten Inhalte von "labor economics" ausgedrückt. Diese beziehen sich auf die Organisation von Arbeitsmärkten, die Funktionsweise und die Ergebnisse des Arbeitsmarktes. Sie umfassen aber auch die Frage der Teilnahme von Personen am Arbeitsmarktgeschehen sowie die unterschiedlichen öffentlichen Politikformen in Bezug auf Beschäftigung und Bezahlung von Arbeitskräften. Die Einbeziehung von organisationstheoretischen Aspekten war in der Vergangenheit weitgehend ausgeblendet. In den letzten Jahren wird das hohe $\mathrm{MaB}$ an Kompatibilität zwischen 'labor economics' und Organisationstheorie deutlich. Weitere Forschungsfelder der "labor economics", die die erwähnten Inhalte ergänzen, basieren auf der Analyse von Arbeitsangebot und Arbeitsnachfrage sowie der Interaktion zwischen beiden (Allokation) und der Entlohnung. 
Die Elemente der Analyse spielen eine Rolle sowohl bei in- als auch externen Arbeitsmärkten.

Im folgenden soll kurz auf den Inhalt bzw. die damit verbundene Fragestellung eingegangen werden. Zunächst deshalb zum Arbeitsangebot: dieses wird durch vier Faktoren bestimmt, die in der Mainstreamliteratur immer wieder angetroffen werden (hier exemplarisch: Hamermesh/Rees, 1988):

a) die Erwerbsbeteiligung, sie umfaßt den Teil der Bevölkerung, der am Erwerbsleben teilnimmt,

b) das Angebot an Arbeitszeil,

c) das $\mathrm{MaB}$ an Leistungsbereitschaft und

d) das Ausbildungs- und Fähigkeitsniveau.

In der neoklassischen Theorietradition liegt dem Arbeitsangebot die Grenznutzentheorie zugrunde, die besagt, daß Arbeitsangebot nach der Einkommens-Freizeit-Präferenz des Arbeitanbieters erfolgt.

Bei der Nachfrage nach Arbeit wird davon ausgegangen, daß Arbeit durch die Unternehmen nachgefragt wird so wie andere Resourcen, die in die Produktion eingehen. Hamermesh/Rees (1988) weisen darauf hin, daß die zugrunde liegende Grenzproduktivitätstheorie trotz massiver Kritik von Seiten der institutionalisten Theorietradition überlebt hat, da bisher noch keine Alternative zur Verfügung steht.

Die Allokation von Arbeit gestattet eine Koordination der 'eigenständig' entworfenen Pläne von Anbieter und Nachfrager. Eine Rolle kann dabei der Markt spielen, von dem in der neoklassischen Theorie unterstellt wird, daß er funktioniert, also keine Einschränkungen vorhanden sind, die das Funktionieren beeinträchtigen.

Arbeitslosigkeit steht als Beispiel (Muysken/Neubourg, 1989; Joehr, 1986; Rothschild, 1980), bei dem die Allokation von Arbeit (vorübergehend) nicht funktioniert. Daneben zählen alle Bemühungen im politischen Umfeld, um durch entsprechende Maßnahmen die Allokation von Arbeit doch noch zustande zu bringen. Der Arbeitsmarkt stellt sich als Arena dar (v. Hoof, 1987).

Schließlich zählt die Entlohnung (Sadowski/Stengelhofen, 1989) zu den wichtigsten Bedingungen für das Zustandekommen der Allokation von Arbeit. Die Mainstreamliteratur spricht von den angemessenen Formen der Bezahlung entsprechend der Leistung und der Qualifikation des Gehaltsempfängers.

Zu diesen Forschungsgebieten besteht ein Reichtum an Literatur und Ergebnissen theoretischer und empirischer Untersuchungen. Dennoch ist dies kein Indikator dafür, daß bestimmte Teilgebiete zureichend erforscht sind.

Viele volkswirtschaftlich orientierte Forschungsaktivitäten konzentrieren sich auf den externen Arbeitsmarkt als Teil der Makroökonomie. Dabei wird in der Theorie vielfach abstrahiert von den Prozessen und der Dynamik, die sich arbeitsmarktpolitisch in Unter- 
nehmensorganisationen vollziehen. Inteme Arbeitsmärkte wurden in der Vergangenheit vielfach als 'black box' angesehen. Dies führt dazu, daß die Wirkungsweise interner Arbeitsmärkte vielfach unterschätzt wird bzw, sich auf Annahmen im Rahmen neoklassischer Modellbildung beschränkt. Verschiedene Disziplinen teilen sich die Erforschung dieses Fachgebietes.

Die betriebswirtschaftliche Fachrichtung läßt sich weiter aufteilen in Personalwirtschaft, Management und Organisationsforschung (Wöhe, 1973; Fombrun, 1984; Staehle, 1985). Wesentliche Impulse kommen von der Soziologie (van lterson, 1989, 1992), insbesondere Arbeits- und Industriesoziologie. Darüberhinaus spielen die Fachgebiete Psychologie und Arbeitsrecht eine Rolle.

Das Fachgebiet Arbeitsmarkt- und Berufsforschung erfuhr seine Entwicklungsimpulse ursprünglich aus einer volkswirtschaftlichen Orientierung. Inzwischen sind deutliche Tendenzen der Konvergenz von volks- und betriebswirtschaftichen Orientierungen testzustellen (Brandes/Liepmann/Weise, 1982). Als Beispiel läßt sich Humankapitaltheorie im Hinblick auf allgemeines Kapital und betriebsspezifisches Kapital anführen.

Diese Untersuchung verfolgt den Anspruch, das Fachgebiet der Arbeitsmarkt- und Beschäftigungsforschung sowie der 'organizational economics' (Douma/Schreuder, 1991) als Teil der Betriebswirtschaftslehre einander näher zu bringen. Beide Forschungsgebiete, wie noch im einzelnen zu zeigen ist, ergänzen sich und führen dazu, daß das Konzept betriebsinterner Arbeitsmärkte zu einer Schlüsselkategorie in der aktuellen Arbeitsmarktforschung (Preisendörfer, 1988) wird. Sie bilden den theoretischen Ausgangspunkt für die Entschlüsselung des Phänomens der internen Arbeitsmärkte.

\subsection{Der 'Markt' für Arbeit als Anpassungsprozeß}

Anpassung und Verteilung repräsentieren die zwei Seilen von Allokation, die nach der neoklassischen Arbeitmarkttheorie in einem Akt erfolgt. Sengenberger (1987) weist auf die Erscheinung hin, daß Anpassungs- und Verteilungsfunktion häufig auseinander fallen. Sie sind vielfach nicht koordiniert und Gegenstand getrennter Handlungen. Die Ursache für diese Erscheinung wird in der Tatsache vermutet, daß der Lohn als Steuerungsregulativ nur begrenzte Wirkung zeigt. Auch Berger/Offe (1984) konstatieren ein 'versagendes Allokationsprinzip' und fordem eine Ergänzungsbedürfigkeit des versagenden Allokationsprinzips. Die Autoren plädieren für die Beibehaltung des klassischen Allokationsprinzips 'Arbeitsmarkt' und sehen die Ergänzung in anderen Prinzipien der Nutzung und Alimentierung von Arbeitskraft.

Der Arbeitsmarkt weist Besonderheiten auf. Der wesentliche Unterschied bei der Durchsetzung von Konkurrenzstrategien auf dem Arbeitmarkt im Gegensatz zum Gütermarkt besteht darin, daß asymmetrische Durchsetzungsbedingungen auf Arbeitsmärkten entstehen, die meistens zugunsten der Nachfrager und zu ungunsten der Anbieter von Arbeit ausfallen.

Ein weiteres Argument (Schmid, 1987), was die Besonderheit des Arbeitsmarktes kennzeichnet, ist, daß das Auftreten von Ware auf dem Markt sich nach Kriterien der Ver- 
käuflichkeit richtet. Bei Arbeitskräften wird das 'Arbeitsangebot' nach demographischen Prozessen, institutionellen Regeln und sozialökonomischen Prozessen geprägt.

Ein weitere Besonderheit des Arbeitsmarktes besteht in der Tatsache, daB die Arbeitskraft für ihren Besitzer ohne den 'Verkauf' weitgehend wertlos bleibt. Dieser Aspekt lenkt die Aufmerksamkeit auf die Kriterien, die die Menge des Arbeitsangebotes bestimmen. Zu nennen sind hier die Zahl der Personen, die in der Gesellschaft anzutreffen sind und die Zahl derjenigen, die ihr Leben durch den Verkauf von Arbeit bestreiten, die also nicht Produktionsmittelbesitzer sind.

"Die marktstrategische Unterlegenheit der Angebotsseite auf dem Arbeitsmarkt hat ihren Grund darin, daß jeder Versuch, die Angebotsmenge der Arbeitskraft kollektiv zu reduzieren, zu einer 'unzumutbaren', organisatorisch nicht durchsetzbaren Vermindenung der Subsistenzmöglichkeiten führen müßte." (Offe/Hinrichs, 1977, S. 12)

Aus der marktstrategischen Unterlegenheit der Angebotsseite am Arbeitsmarkt werden Maßnahmen von staatlicher Seite begründet, die das Ziel verfolgen, durch Schutzgesetze und Mindesteinkommen die 'freie Entfaltung' der Marktkräfte zu beschränken. Eine im Zeitablauf auftretende weitere Asymmetrie besteht in der Tatsache, daß durch produktivitätssteigande Innovationen die Nachfrage nach Arbeitskräften abnimmt.

Aus der Reihe der hier aufgeführten Argumente läßt sich ableitcn, daß der Arbeitsmarkt nur beschränkt mit Warenmärkten vergleichbar ist.

Sowohl über den externen als auch den internen Arbeitsmarkt werden Anpassungsleistungen im Sinne der Reallokation von Arbeitskraft vollzogen. Besonders aus makroökonomischer Sicht wird die Anpassungsfähigkeit auf Grund von Lohn- und Beschäftigungsstarrheiten bezweifelt. In diesem Abschnitt sollen kurz Parameter der Anpassung und Anpassungspolitik benannt werden. Sengenberger (1987) zeigt drei Parameter der Anpassung auf, die sowohl eine Mikro- als auch Makrodimension (Kalleberg, 1989) umfassen. Die Parameter bestehen aus Arbeitsangebot, Arbeitsnachfrage und der Zuordnung von Angebot und Nachfrage. Ihre Gestaltung sowie schließlich die Zuordnung von Angebot und Nachfrage (Allokation) machen Anpassung auf dem Arbeitsmarkt möglich. Den jeweiligen Parametern ordnet Sengenberger betriebsorientierte als auch allgemeinere Politikfelder zu, über die sich die Gestaltung der Anpassung vollziehen kann.

Von Anpassungsproblemen handelt auch die sogenannte Deregulierungsdebatte. Zum Verständnis dieser Debatte ist zu sagen, daß die Impulse von makroökonomischer Seite ausgehen. Behauptet wird, daß Vollbeschäftigung und Preisstabilität durch Lohn- und Beschäftigungsrigiditäten verhindert werden (Doeringer/Piore, 1985). Unter anderem wird dabei an interne Arbeitsmärkte gedacht, die von ihrer Anlage so konstitutiert sind, daß ein negativer Effekt auf die Vollbeschäftigung entsteht. Die Forderung nach 'Deregulierung' zielt auf die bessere Anpassungsfähigkeit der Arbeitsmärkte an wirtschaftliche Veränderungen. Befürworter dieser 'Bewegung' beabsichtigen, mehr 'Markt am Arbeitsmarkt' zu erreichen bzw. eine Rücknahme staatlicher Aktivitäten. Die zugrundeliegenden Ideen basieren auf der neoklassischen Modellwelt der vollkommenen Konkurrenz und des 
Gleichgewichts. Keller (1990, S. 370) setzt sich kritisch mit dem 'Marktparadigma' auseinander.

Die Wirkungen von Deregulierungsstrategien werden vielfach in Zweifel gezogen, solange noch keine empirischen Belege für die angeblichen Effekte vorliegen. Sengenberger (1987) sieht keinen Handlungsbedarf im Sinne des 'Marktparadigmas', da er von einem großen Anpassungs- und Austauschpotential intemer Arbeitsmärkte ausgeht.

Die aus makroökonomischer Perspektive behaupteten Rigidiäten am Arbeitsmarkt abstrahieren von der in der Realität anzutreffenden und teilweise auf Konsens basierenden (historisch gewachsenen) Institutionen des Arbeitsmarktes, die, wie noch im einzeinen zu zeigen ist, über die erforderlichen Anpassungspotentiale verfügen.

\subsection{Begriff und Funktionsweise von Arbeitsmärkten}

Die oben angeführten Überlegungen stecken einen Rahmen ab für eine eingehendere Betrachtung der Dynamik des Arbeitsmarktsgeschehens auf mikroökonomischer Ebene. Hier sollen zunächst die definitorischen Grundlagen vorgestellt werden.

In- und externe Arbeitsmärkte sind Teile eines Arbeitsmarktes. Sengenberger (1987, S. 31) definiert den Begriff Arbeitsmarkt als gesellschaftliche Einrichtung, in der zwei Funktionen und Prozesse miteinander verbunden sind:

(1) "Die Vermittlung von Angebot und Nachfrage von Arbeitskraft; diese sei als Anpassung bezeichnet; sie umschlieBt nicht allein die (veränderte) Zuordnung von Arbeitskräften zu Arbeitsplätzen, sondem auch die Veränderung von Zahl und Art der Arbeitsplätze und Arbeitskräfte. Der Begriff Anpassung impliziert nicht, daß Vermittlung von Angebot und Nachfrage notwendig zu einer Gleichgewichtslage im Sinne der Räumung des Arbeitsmarktes führt. Anpassung schließt den Fall des Ungleichgewichts mit ein.

(2) Die Verteilung gesellschaftlicher Chancen und Risiken auf die Arbeitskräfte, verstanden nicht nur als Einkommen im materiellen Sinne (wie Löhne und andere geldwerte Gratifikationen), sondem auch im immateriellen oder nicht unmittelbar materiellen Sinne von Beschäftigungssicherheit, Autonomie der Arbeitsgestaltung, gesellschaftlichem Status und sozialem Ansehen, berufliche Entwicklungsmöglichkeiten etc."

Dieser umfangreichen Definition von Arbeitsmarkt soll die des in- und extemen Arbeitsmarktes als spezifischere Ausprägung der Globalperspektive 'Arbeitsmarkt' von Doeringer/Piore (1971) vorgestellt werden. Der interne Arbeitsmarkt ist definiert als:

"... an administrative unit, such as a manufacturing plant, within which the pricing and allocation of labor is governed by a set of administrative rules and procedures" (Doeringer/Piore, 1971; S. 1/2).

Der Begriff des internen Arbeitsmarktes ist recht global definiert. Im Begriff kommt nur unzureichend die Vielzahl der Merkmale zum Ausdruck, die elementar sind für einen Sammelbegriff. Diese Definition dient zunächst vorläufig zur ersten Orientienung. 
Der externe Arbeitsmarkt kommt zum Ausdruck in der "... conventional economic theory where pricing, allocation and training decisions are controlled directly by economic variables" (Doeringer/Piore, 1971; S. 2). Zwischen dem in- und externen Arbeitsmarkt gibt es Wirkungszusammenhänge, auf die im weiteren Verlauf der Arbeit eingegangen wird.

Das Konzept vom in- bzw, externen Arbeitsmarkt ist eingebettet in der sogenannten 'dual labor market'-Hypothese. Diese Hypothese basiert auf einer Aufteilung der Ökonomie in zwei Teile (Averitt, 1968), einem primären und sekundären Sektor. Der primäre Sektor wird mit guten Beschäftigungsmöglichkeiten assoziiert, der sekundäre Sektor mit schlechteren.

Die Definitionen von Sengenberger und Doeringer/Piore sind weniger geeignet für eine empirische Umsetzung der zentralen Fragestellung im Rahmen dieser Untersuchung. Die Definitionen erfordern eine konzeptionelle Ergänzung, die über die Definitionen hinausgeht. Einen Ansatzpunkt dazu bielet Lutz (1987). Mit Lutz Konzept wird die Perspektive auf den untemehmensintemen Arbeitsmarkt gelenkt. Damit richtet sich die Aufmerksamkeit auf die Erschließung der 'Infrastruktur' interner Arbeitsmärkte.

Lutz kombiniert die Konzepte 'interner Arbeitsmarkt' und 'betriebszentrierte Arbeitsmarktsegmentation'. Der interne Arbeitsmarkt basiert als umfassenderer Begriff auf drei von insgesamt fünf definierten Merkmalen betriebszentrierter Arbeitsmarktsegmentation. Diese beruhen auf 'ports of entry' für neue Arbeitnehmer, einer arbeitsplatzbezogenen Qualifikations-, Verantwortungs- und Verdienstabstufung sowie auf hierarchiebezogene Mobilitätsmuster. Betriebszentrierte Arbeitsmarktsegmentation ist dann gegeben, wenn statt der beim internen Arbeitsmarkt gegebenen Betriebsbindung eine wechselseitige Bindung zwischen Betrieb und Beschäftigten entsteht. Außerdem muß das Allokationmuster gegenüber den Beschäftigten ein hohes $\mathrm{Maß}$ an Verbindlichkeit aufweisen.

Lutz verbindet die Definition des internen Arbeitsmarktes mit konzeptionellen Perspektiven, die die Charakteristiken intemer Arbeitmärkte ansprechen. Dieser Ansatz bedarf der konzeptionellen Konkretisierung im Hinblick auf die komplexen Beschäftigungsstrukturen intemer Arbeitsmärkte. Dies ist Gegenstand der theoretischen Teils dieser Untersuchung.

\section{$1.4 \quad$ Zentrale Fragestellung}

Aus der groben fachgebietsbezogenen Aufteilung des Forschungsgebietes wurden erste Ansätze zur Konkretisierung des Forschungsgegenstandes deutlich. Es geht um einen Beitrag bestehend aus einer integrativen Sichtweise mikrostruktureller Allokation. Dabei werden Organisationstheorie und die Theorie intemer Arbeitsmärkte einander nähergeführt. Als zentrale Fragestellung und erkenntnisleitendes Interesse der Untersuchung steht im Mittelpunkt:

In welchem Maß unterscheiden sich Maschinenbürokratien und professionelle Bürokratien in der Prägnanz der Anwesenheit der angegebenen Beschäftigungskennzeichen von internen Arbeitsmärkten? 
Die zentrale Fragestellung entspricht dem Erkenntnisinteresse, die vielfältigen Formen interner Arbeitsmärkte aus organisationstheoretischer Sicht der Analyse zugänglich zu machen. Zwei organisationstheoretische Strömungen beherrschen die zentrale Fragestellung in ergänzender Weise. Dies sind einerseits 'governance structures', die auf Williamsons Transaktionskostenansatz (1981) zurückgehen und die interne Beherrschung von Organisationshierarchien kennzeichnen, andererseits die Kontingenztheorie mit den Organisationskonfigurationen von Mintzberg (1979, 1983, 1989), der die Heterogenität von Organisationen auf scchs Organisationskonfigurationen zu reduzieren versucht, von denen zwei im Rahmen dieser Studie untersucht werden. Es handelt sich um die Organisationskonfiguration der 'Maschinenbürokratie' und der 'professionellen Bürokratie'.

Die sich ergänzenden Theorieströmungen erlauben eine unterschiedliche Sichtweise interner Arbeitsmärkte. Außerdem tragen sie dazu bei, die in diesem Zusammenhang relevanten strukturbezogenen Beschäftigungsmerkmale empirisch und konzeptionell zu erschließen.

Die Maschinenbürokratie ist im Sinne Webers (1972) die klassische bürokratische Organisationskonfiguration (Crozier, 1964). Das Image dieser Organisationsform entspricht dem einer maschinellen Funktionsweise (Morgan, 1986). Diese besteht in einer rigiden Struktur, bei der die Koordination der Aufgaben durch die Standardisienung der Arbeitsprozesse erreicht wird. Diese Organisationskonfiguration ist horizontal und vertikal spezialisiert.

Die professionelle Bürokratie ist eine Organisationsform, deren Koordinationsmechanismus aus der Standardisierung der Aufgaben besteht. Dies bedeutet, daß die Hauptfunktionen auf Wissen und Professionalität der Mitglieder dieser Organisation beruhen. Die jeweilige Qualifikation der Organisationsmitglieder wurde extern erworben in formalisierten und anerkannten Ausbildungseinrichtungen. Diese Organisationskonfiguration ist horizontal spezialisiert und eröffnet den Mitgliedem die Chance zur Aus- und Weiterbildung. Formalisierung ist auf einen geringen Grad reduziert. Beschäftigte in dieser Organisation verfügen über einen hohen Grad an Autonomie. In Kapitel 3 wird der Bezug zwischen 'govemance structures' und Organisationskonfigurationen hergestellt.

Bei den strukturbezogenen Beschäftigungsmerkmalen von Arbeitsorganisationen sollen jene angesprochen werden, die eine allokationsrelevante Bedeutung haben in Bezug auf Strukturen (Infrastruktur) intraorganisationaler Mobilität von Arbeitnehmem und die Besetzung offener Stellen mit Orientierung auf den intemen Arbeitsmarkt.

Nach Williamson (1975) lassen sich Wirtschaftsaktivitäten grob gesehen entweder über den Markt oder über die Hierarchie eines Untemehmens (Organisation) abwickeln. Beim internen Arbeitsmarkt wird bei aller Widersprüchlichkeit, die die Definition des Begriffs 'interner Arbeitsmarkt' ausmacht (Windolf/Hohn, 1984), die 'Hierarchieseite' angesprochen.

'Governance structures' sind nach Williamson (1979, S. 239) "... the institutional matrix within which transactions are negotiated and executed." Die Transaktionen beziehen sich auf menschliche Arbeit innerhalb von Organisationen. 
Die Organisationskonfiguration der 'Maschinenbürokratie' und der 'professionellen Bürokratie' repräsentieren unter anderen zwei Hierarchiealternativen. Beide Organisationskonfigurationen zeigen eine unterschiedliche Nähe zum Markt. Die folgende Skizze soll dies verdeutlichen.

Abb. 1: Hypothetische Position der Organisationen im Spektrum Hierarchie/Markt

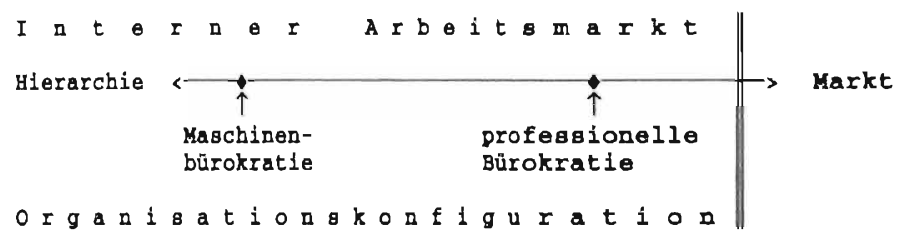

Ohne der ausführlichen Darstellung der Organisationskonfigurationen vorweg zu greifen (Kapitel 3) kann der inteme Arbeitsmarkt - vereinfachend gesehen - zwei Formen annehmen. Dabei handelt es sich um die Organisation der Maschinenbürokratie und der professionellen Bürokratie. In der hypothetischen Positionsbestimmung ist die Maschinenbürokratie mehr hierarchieorientiert, während die professionelle Bürokratie mehr 'marktnah' im Rahmen des obigen Spektrums zu lokalisieren ist. Eine differenziertere Perspektive zur Geschlossenheit bzw. Offenheit von Organisationen wird in Soeters/Schwan (1993) entwickelt.

Althauser/Kalleberg (1981, S. 129 ff.) vertreten einen Arbeitsmarktforschungsansatz. Ausgangspunkt ist die Idee, das mehrere unterschiedliche Arbeitsmarktformen innerhalb einer Organisation existieren können. Grundlage der Differenzierung der Arbeitsmarktformen innerhalb einer Organisation sind drei Wesensmerkmale interner Arbeitsmärkte. Diese bestehen aus der Jobleiter, dem Eintritt im unteren Hierarchiebereich sowie der Mobilitätsentwicklung über die Jobleitern.

Im Falle des sogenannten furmenintemen (firm internal labor market (FILM)) Arheitsmarktes treffen alle Wesensmerkmale zu. Daneben kann ein berufsfachlicher interner Arbeitsmarkt (occupational internal labor market (OILM)) unterschieden werden, der dem firmeninternen in verschiedenen Kriterien ähnelt. In Kapitel 3 soll näher eingegangen werden auf das Konzept von Althauser/Kalleberg. Das Konzept Althauser/Kallebergs konzentriert sich hier auf die oben genannten zwei Formen der Arbeitsorganisation.

\subsection{Weiterer Aufbau der Studie}

Der weitere Aufbau der Studie stellt sich wie folgt kurz dar: das folgende Kapitel (2) inventarisiert die zentralen Aussagen in Bezug auf interne Arbeitsmärkte basierend auf der Studie von Doeringer/Piore (1971). Analytisch erschlossen werden nach der Theorie des intemen Arbeitsmarktes Stukturdimensionen der Beschäftigung. Ziel dabei ist es, Aufschluß über die Konstitution intemer Arbeitsmärkte zu erhalten.

Kapitel 3 geht ein auf Bedingungen der Existenz interner Arbeitsmärkte. Dann werden die in diesem Kapitel angesprochenen zwei organisationstheoretischen Ausgangspositionen 
dargestellt und konzeptionell spezifiziert. Im Vordergrund steht dabei die Frage, inwieweit 'governance structures' bzw. der Organisationskonfigurationen von Mintzberg vergleichbzw. integrierbar sind hinsichtlich der Beschäftigungsstrukturkennzeichen intemer Arbeitsmärkte. Dabei handelt es sich - wie angedeutet - um die Transaktionskostentheorie und Kontingenztheorie. Dieses Kapitel endet mit der Erschließung von strukturbezogenen Beschäftigungsmerkmalen spezifiziert auf die jeweilige Organisationskonfiguration sowie auf die beschäftigungsrelevante Infrastruktur der Organisationskonfiguration.

Im Mittelpunkt von Kapitel 4 steht die zentrale Fragestellung der Untersuchung sowie einige erkenntnisleitende Hypothesen als Ausarbeitung der zentralen Fragestellung.

Kapitel 5 gibt Auskunft über die benutzten Methoden der empirischen Untersuchung, insbesondere der Datenerhebung. Außerdem werden die benutzten Methoden verantwortet.

Im sechsten Kapitel werden die untersuchten Organisationen qualitativ beschrieben aufgrund des gewonnenen empirischen Materials. Daneben werden in organisationsvergleichender Weise Beschäftigungskennzeichen gegenübergestellt.

Die Resultate der empirischen Untersuchung werden im Kapitel 7 vorgestellt. Gegenstand dieser Untersuchung ist die Erschließung organisationsspezifischer Beschäftigungsmerkmale aus der Perspektive intemer Arbeitsmärkte in Form von Teilanalysen. Diese bestehen aus einer Beförderungs-, einer Gehaltsentwicklungs- sowie einer Beschäftigungsdaueranalyse.

Das folgende Kapitel 8 ist ebenfalls den Resultaten der empirischen Analyse vorbehalten. Dabei handelt es sich um die Resultate einer offenen Stellenanalyse, die die Erkenntnisse der organisationsinternen Allokation von Arbeit ergänzen soll. Sowohl für die Analysen in Kapitel sieben als auch die Analyse der Besetzung offener Stellen gilt eine organisationsvergleichende Betrachtung der Organisationsform der Maschinenbürokratie und der professionellen Bürokratie in Anlehnung an Mintzberg.

Schließlich endet die Untersuchung mit Schlußfolgerungen aus den zentralen Erkenntnissen, einer Theorierückkoppelung und gesellschaftlichen Aspekten zur Entwicklung interner Arbeitsmärkte in Form von Kapitel 9.

Die Bedeutung der empirischen Untersuchung ist in der integrierten Sichtweise von Arbeitsmarkt und Organisation zu sehen. Die Organisationsperspektive ermöglicht die Erschließung einer organisationsspezifischen Untemehmens- (Organisations-) infrastruktur hinsichtlich der Beschäftigung von Arbeitnehmem. Abgeleitet werden organisationsbezogene Beschäftigungsmuster, die Aussagen erlauben über die untemehmensinteme Allokation.

Die empirische Untersuchung soll Aufschluß bieten über die Beschäftigungsmerkmale von zwei Organisationskonfigurationen. Erschlossen werden Unterschiede und Gemeinsamkeiten der Ausprägung von Beschäftigungskennzeichen interner Arbeitsmärkte. 
Der 'rote Faden' der Untersuchung stellt sich im Kurzüberblick wie folgt dar. Kapitel 1 enthält mit der Einleitung eine Übersicht in die Thematik, legt die begrifflichen Voraussetzungen und spricht die zentrale Fragestellung an. Kapitel 2 geht ein auf institutionelle Theorien interner Arbeitsmärkte, spricht die Funktionsweise an und erschließt die Infrastruktur intemer Arbeitsmärkte mit Hilfe von Strukturdimensionen. In Kapitel 3 wird der organisationstheoretische Bezug gelegt. Hier wird an Hand der für die Organisation relevanten Beschäftigungskennzeichen untersucht, inwieweit diese mit den Stukturdimensionen interner Arbeitsmärkte korrespondieren. Gekoppelt an Kapitel 4 sind Hypothesen, die auf der zentralen Fragestellung basieren. Kapitel 5 geht ein auf die Untersuchungsmethoden. In Kapitel 6 werden die untersuchten Organisationen vorgestellt. Kapitel 7 und 8 repräsentieren die Resultate der empirischen Untersuchung. Kapitel 9 faßt die wichtigsten Ergebnisse zusammen. 
"You must realize that the labor market is like the marriage market." (Boulding, 1951, S. 254)

\section{2}

\section{Arbeitsmarktstrukturdimensionen in Theorien interner Arbeitsmärkte}

In diesem Kapitel geht es um die Strukturdimensionen in Theorien intemer Arbeitsmärkte und deren Einordnung in die jeweiligen Theorieströmungen. Zwei Theorieströmungen werden dabei berücksichtigt: es handelt sich zum einen um die neoklassische Arbeitsmarkttheorie, die hier nur kurz angesprochen werden soll. Sie steht für eine Anzahl von Theoriezweigen, die den intemen Arbeitsmarkt zum Gegenstand haben. Zum anderen geht es um die institutionalistische Theorieströmung und deren Erklärungspotential in Bezug auf den intemen Arbeitsmarkt.

Beide Theorieströmungen haben voneinander Impulse erfahren und sich gegenseitig befruchtet, die zu einer weiteren Erschließung des Forschungsfeldes führten. Beide Theorien haben sich ebenfalls behauptet und ihr Profil erweitern können.

Beispiele für die Fortentwicklung der neoklassischen Arbeitsmarkttheorie zeigen einige Theorien wie die Insider-Outsider-Theorie (Lindbeck/Snower, 1988), die Efficiency-WageTheorie (Carmichael, 1990; Fehr, 1989; Gerlach/Hübler, 1989; Bellmann, 1986; Akerlof/ Yellen, 1986) sowie die implizite Vertragstheorie (Wolfs, 1992). Sie stehen exemplarisch für die Weiterentwicklung neoklassisch geprägter Theorien. Es handelt sich um Theorien, die in Bezug auf den internen Arbeitsmarkt einen Erklärungswert haben (Schwan, 1992a).

In diesem Kapitel wird ausführlicher eingegangen auf die andere Theorieströmung, nämlich die institutionalistisch geprägte mit der Zielsetzung, den Beitrag für die Erklärung von Strukturdimensionen des internen Arbeitsmarktes zu prüfen. Dieser Schritt erscheint deshalb erforderlich, um in einem späteren Stadium dieser Untersuchung den Bezug zu den Organisationskonfigurationen aus der Perspektive des intemen Arbeitsmarktes herzustellen. Die Rekonstruktion von Arbeitsmarktstrukturdimensionen aus der Theorie des internen Arbeitsmarktes nach Doeringer/Piore dient als Ausgangspunkt für die vergleichende Betrachtung organisationsbezogener Beschäftigungskennzeichen, die im nächsten Kapitel aus Organisationstheorien abgeleitet werden. Die Erschließung von Arbeitsmarktstrukturdimensionen entspricht $z$ wei Bedürfnissen. Zum einen geht es um die Reduzierung der Komplexität intemer Arbeitsmärkte sowie die Funktionsweise, zum anderen um die ErschlieBung von organisationsstrukturbezogenen Beschäftigungsmerkmalen als Ausgangsvoraussetzung für die empirische Untersuchung. 


\subsection{Interne Arbeitsmärkte aus institutionalistischer Arbeitsmarkttheorieperspektive}

Die institutionalistische Arbeitsmarktanalyse läßt sich exemplarisch mit den Namen Clark Kerr, John Dunlop und John Commons assoziieren. Eines der wesentlichen Kennzeichen der institutionalistischen Denkrichtung ist die Überwindung des traditionellen Ökonomismus basierend auf dem Rationalitätsaxiom und des nutzenmaximierenden Wirtschaftssubjekts (Pfriem, 1979). Später setzte sich der Sammelbegriff 'Segmentationstheoretiker' durch. Cain (1976, S. 1215) zeigt auf, welche Theoretiker mit dem Begriff angesprochen werden.

"Among the many names given to these dissident theories of the labor market are the following: radical, dual (primary, secondary), tripartite (core, peripheral, irregular), stratified, hierarchical, and job competition. Also, the concept of a dichotomy between 'internal' and 'external' labor market is related to the dual and tripartite theories. It often will be expedient to lump these different (but overlapping) theories together in focusing on their criticsms of orthodox theory."

Die 'zentralen Glaubenssätze' der 'institutional economics' (Soeters/Keizer, 1987) bestehen aus drei Elementen (Ramstad, 1981, S. 341): "...behaviorism, institutional balance, and inductive reasoning." Das erste Element berücksichtigt die Erklärung individuellen Verhaltens als Resultat und Einfluß sozialer Institutionen. Das zweite Element basiert auf der Tatsache, daß nicht Marktkräfte sondern Institutionen die Zusammenarbeit innerhalb von Organisationen beeinflussen (Schmid, 1988). Schließlich bleibt als weiteres Element eine ständige Reflexion des Verhaltens ökonomischer Einheiten im Rahmen induktiver Ableitung. Gedacht wird dabei beispielsweise an Arbeitsmarktsegmente und die Erschließung der dazu gehörenden Subeinheiten. Becker (1985) kritisiert, daß Ramstad keine eindeutigen Kriterien zur Bestimmung der institutionalistischen Arbeitsmarktanalyse benennen kann. Aufgezeigt werden soll damit das Dilemma des Identifikations- und Abgrenzungsproblems einer institutionalistischen Arbeitsmarktanalyse (Schudlich, 1987).

Ohne weiter im Detail darauf einzugehen soll anhand der von Freiburghaus (1979) gegenüber gestellten Wissenschaftsauffassung Poppers (1972) ein Blick auf die fundamentalen Unterschiede geworfen werden.

Popper (1972) kritisiert ein Wissenschaftsverständnis als "Kübeltheorie des menschlichen Geistes", womit gemeint ist, daß der Wissenschaftler die konkrete ganze Realität möglichst zum Gegenstand seines Erkenntnisinteresses machen möchte. Freiburghaus (1979, S. 165 ff.) stellt im Rahmen eines Theorievergleichs auf der Basis der Aussage von Popper den Bezug her zur institutionalistischen Theorie. Das Resultat sind dann holistische Erklärungsansätze. Der neoklassische Theorieansatz wird mit einer "Scheinwerfermethode" verglichen. Damit wird zum Ausdruck gebracht, daß die wissenschaftliche Aktivität sich auf endogene Variablen konzentriert unter Vemachlässigung von exogenen Variablen der Modellbetrachtung. Die Kritik der Institutionalisten gipfelt in der Aussage, daß die neoklassische Theorie "l'art pour l'art" sei, die keinen Bezug zu den 'echten Problemen' vermittelt. 
Aktuelle Untersuchungen (Ross, 1981; Becker, 1985; Singer, 1986) zeigen, daß sowohl das neoklassische als auch das institutionelle Paradigma nicht isolier ist durch Ignorierung des jeweils anderen Paradigmas. Vielmehr haben sich integrative Verknüpfungen ergeben (beispielsweise Okun, 1981 und Hodgson, 1988), die einen positjven Effekt auf die Arbeitsmarktforschungskultur hatten und haben.

\subsubsection{Der Ansatz von Kerr}

So wird im folgenden auf ein institutionelles Konzept zurückgegriffen, das die Entstehung und Entwicklung der Segmentationstheorien und die damit verbundenen Erkenntnisse wesentlich mit beeinflußt hat.

Es handelt sich um die 'Balkanisierung' von Arbeitsmärkten. Dem liegt die Vorstellung zugrunde, daß zahlreiche verschiedenartige Barrieren die Gesamtheit der Beschäftigungsverhältnisse aufteilen. Kerr (1954, S. 96) nennt fünf Gründe für die Aufteilung in Teilmärkte: "(1) the preferences of individual workers, (2) the preferences of individual employers, (3) the actions of the community of workers, (4) the actions of the community of employers, and (5) the actions of govemment.

Von allen fünf Aufteilungsgründen gehen Arbeitsmarktstrukturen und institutionelle Regeln aus, die Kerr dazu veranlaßten, institutionalisierte von nicht institutionalisierten Arbeitsmärkten zu unterscheiden. Instititutionalisiente Arbeitsmärkte lassen sich wie folgt charakterisieren (Kerr, 1954, S. 93):

"Their dimensions are set not by the whims of workers and employers but by rules, both formal and informal. These rules state which workers are preferred in the market or even which ones may operate in it at all, and which employers may or must buy in this market if they are to buy at all. Institutional rules take the place of individual preferences in setting the boundaries."

Die Grenzzichung bzw. -setzung durch institutionelle Regeln bzw. Regulierungen bewirkt eine Zahl von Märkten bzw. Teilmärkten. Institutionelle Regeln bringen Struktur in Arbeitsmärkte. Diese Perspektive fließt aus der Differenzierung Kerrs zwischen - grob gesagt - zwei Arbeitsmarkttypen, dem strukturlosen und dem strukturierten Arbeitsmarkt. Als weitere Differenzierung des strukturierten Arbeitsmarktes erfolgt dann die Aufteilung in 'interne und externe Arbeitsmärkte'. Zwischen in- und externen Arbeitmärkten sorgen 'institutionalisierte Regeln' für eine stärkere Grenzziehung. Definitorisch legt Kerr (1954, S. 101, Fußnote 16) sich folgendernaßen fest:

"The internal market may be the plant or the craft group, and preferment within it may be based on prejudice or merit or equality of opportunity or seniority or some combination of these. The external market consists of clusters of workers actively or passively available for new jobs lying within some meaningful geographical and occupational boundaries, and of the port or ports of entry which are open or are potentially open to them."

Bei der Betrachtung der Definition fältt auf, daß sie Pate gestanden hat für die im ersten Kapitel vorstellte Definition des internen Arbeitsmarktes nach Doeringer/Piore. Nach der 
Definition Kerrs befinden sich auf dem extemen Arbeitsmarkt sämtliche Arbeitskräfte, die potentiell bereit sind, den Zugang zum intemen Arbeitsmarkt zu suchen.

Das besondere dieses Ansatzes liegt in seinem analytischen Wert. Kerr unterbreitet ein Konzept, daß ein Spektrum strukturorientierter Durchdringung umfaBt vom extemen Arbeitsmarkt bis zum einzelnen Arbeitsplatz als Analyseeinheit. Kerr nimmt Abstand von der Betrachtung des Arbeitsmarktes als 'Spotmarket', einer Vorstellung, die in seiner Zeit vorherrschend war. Becker (1985) bezweifelt die analytische Fruchtbarkeit von Kerrs Konzept. Er begründet dies mit Kerrs Präferenz für die Aufteilung in gröbere Klassifikationskategorien, beispielsweise der Einteilung in institutionalisierte und nicht institutionalisierte Arbeitsmärkte. Tatsächlich leuchtet Kerr die Möglichkeiten seines Ansatzes aus, die das Potential der analytischen Verknüpfung bieten von der Makroperspektive Arbeitsmarkt bis hin zur Mikroperspektive des einzelnen Arbeitsplatzes. Eine Schwäche glaubt Becker hinter dem Faktum zu entdecken, daß institutionelle Faktoren bzw. Institutionen unzureichend definiert bzw. spezifiziert werden. Dies kann bei der Vorstellung eines analytischen Konzepts zunächst nicht geleistet werden. Dieses Problem wird dann akut, wenn die empirische Ausfüllung erfolgen soll.

Das Konzept der Balkanisierung (Kerr, 1954) von Arbeitsmärkten ist in hohem Maße mit organisationstheoretischen Ansätzen kompatibel wie noch im einzelnen zu zeigen ist. Interne Arbeitsmärkte haben ein theoretisches Fundament in den Segmentationsansätzen. Die Balkanisierung von Arbeitsmärkten umschreibt die Entstehung von Segmenten und Segmentierung. Eine kurze Einschätzung des Wertes der Segmentationsansätze aus einer mehr zeitnahen Perspektive soll diesen Abschnitt abrunden.

\subsubsection{Sengenbergers Segmentationsansatz}

Mit Sengenberger (1987, S. 52) soll kurz auf die Leitvorstellung der Segmentation eingegangen werden:

"Leitvorstellung der Segmentation ist, daß sich der Gesamtarbeitsmarkt in eine Reihe von Teilarbeitsmärkten aufgliedert, die eine innere Struktur aufweisen, mehr oder weniger gegeneinander abgeschirmt sind, möglicherweise auch mit unterschiedlichen Anpassungsformen und -instrumenten verknüpft sind und unterschiedliche Einkommens- und Beschäftigungschancen aufweisen."

Auf der Basis dieser Leitvorstellung wird die Heterogenität deutlich, die mit den Segmentationsansätzen verbunden ist. Schon hier wird die Unterschiedlichkeit des Bedeutungsgehaltes der Segmentation deutlich. Sengenberger (1987, S. 54) betont, daB in Bezug auf die Grundidee der Differenzierung des Arbeitsmarktes in Teilmärkte bzw. Segmente noch Konsens zu erzielen ist. Bei der Betrachtung des Segmentierungsprozesses fallen nach Sengenberger (S. 63) folgende Entwicklungen auf, die die weitere 'Subsegmentation' befördem und damit zu Inhomogenitäten bei Beschäftigungsgruppen führen. Dazu bei tragen die wechselseitige Verstärkung von Angebot und Nachfrage, die selektive Zuteilung von Arbeitnehmem auf Arbeitsplatzgefüge und schlieBlich die Interaktion von Mikro- und Makroebene des Arbeitsmarktes. Als Resultat der letzten Komponente entstehen stabile 
Arbeitsmarktstrukturmuster. Sie erfahren eine Verstärkung durch die verschiedensten Formen der Institutionalisierung.

Sengenberger spricht von einer Multisegmentation und unterstreicht damit die Aufteilung in verschiedene Dimensionen. Eine kritische Bewertung der Segmentation am Arbeitsmarkı läßı folgende Schlußfolgerungen (Sengenberger, 1987, S. 70) zu:

"Dies bedeutet, daß der Arbeitsmarkt mehrfach geteilt ist, daß Teilungen innerhalb des Geteilten erfolgen. Jede Teilung selbst besteht in einem Akt, der eine Dichotomie hervorruft, aber aus wiederholter Teilung (in Raum und Zeit) entstehen immer mehr abgestuft, kontinuierlicher erscheinende Verteilungsstrukturen. Je mehr Teilungen, desto komplexer wird das Ungleichheitsprofil. Das Bild einer Kaskade mil mehr oder weniger großen Gefällstrukturen, die symbolisieren, wie schwer oder leicht die Zugangsbarriere überwindbar ist, liefert deshalb eine adäquate Beschreibung der Segmentation des Arbeitsmarktes als das duale Modell."

Diese Aspekte sollen um eine andere Perspektive bereichert werden. Biller (1989, S. 179) kommt zu der SchluBfolgerung, daß

"... der von den Segmentationstheorien bisher erarbeitete Differenzierungsgrad nicht genutzt (wurde, R. S.)..." .

Diese Perspektive wird bei Biller (1989, S. 180) durch die folgende Aussage verstärkt:

"Doch selbst wenn die je variantenspezifisch angelegten theoretischen Möglichkeiten voll genutzt werden (wie am ehesten noch von Piore), kann das modelltheoretisch eingebaute Defizit einer vorgängig festgelegten Segmentetypologie und der daraus folgenden $\mathrm{zu}$ geringen Differenzierungskraft (mehr als höchstens drei Segmente stehen nicht zur Verfügung) nicht eleminiert werden."

Die aufgezeigten Perspektiven machen deutlich, warum sich auf der Basis der Segmentationsansätze kein schlüssiges Arbeitsmarktstrukturkonzept ableiten läßt. Zwei Gründe tragen dazu bei. Einerseits bewegt sich die Betrachtung auf der Ebene von Segmenten, ohne daß eine durchgängige Strukturierung der Untereinheiten betrieblicher Arbeitsmärkte erreicht werden kann, andererseits sorgen instititutionelle Momente für eine Komplexität, die den empirischen Zugang erschweren. Diese Einsicht lenkt die Aufmerksamkeit auf die Analyse der Funktion und strukturellen Erscheinung intemer Arbeitsmärkte, die Gegenstand des folgenden Abschnitts sein wird.

\subsection{Die Funktionsweise von internen Arbeitsmärkten}

Die im vorhergehenden Abschnitt gewonnene Erkenntnis, daß über die Segmentationsansätze nur unzureichend die Arbeitsmarktstrukturdimensionen zu erschließen sind, richtet die Aufmerksamkeit auf den Erklärungswert der Theorie des intemen Arbeitsmarktes. In diesem Abschnitt werden einige jener Arbeitsmarktheorien vorgestellt, die die Funktionsweise von internen Arbeitsmärkten erklären. Kurz dargestellt werden die wichtigsten Theorieelemente und -inhalte. Das zentrale Erkenntnisinteresse ist konzentriert auf die 
betriebliche Arbeitsmarktstruktur und die jeweilige konzeptionelle Ausfüllung im jeweiligen Theoriekontext. Eine kurze Bewertung der jeweiligen Theorie schließt sich an.

\subsubsection{Theorieansätze zur Erklärung interner Arbeitsmärkte und deren Beitrag im} Rahmen von Arbeitsmarktstrukturdimensionen

Aus der großen Fülle der Theorien zur Erklärung interner Arbeitsmärkte sollen einige hier angesprochen werden. In den Vordergrund rückt die Theorie intemer Arbeitsmärkte nach Doeringer/Piore als den auf die Gesamtheit der konstitutierenden Bedingungen interner Arbeitsmärkte weitestgehenden Ansatz. Begründet wird dies durch einen weitgehenden Konkretisierungsgrad. Daneben werden die Ansätze von Lutz und Sengenberger betrachtet als Repräsentanten, deren Zeitperspektive mehr zur Aktualität hin orientient ist.

Markiert werden soll dabei der Erklärungsansatz bzw. die Theorietradition, dem die jeweilige Theorie entstammt. Oft stehen die Theorienvarianten von ihrem Erklärungsansatz in Konkurenz zu der 'Grundtheorie' intemer Arbeitsmärkte von Doeringer/Piore. Doch zunächst sollen zur Orientierung und besseren analytischen Erfassung (und Beurteilung) in Anlehnung an die Theore intemer Arbeitsnärkte (Doeringer/Piore, 1971) Arbeitsmarktstrukturdimensionen (-ebenen) abgeleitet werden mit dem Ziel, auf der Basis von Arbeitsmarktstrukturen interne Arbeitsmärkte zu erschließen. Damit wird eine Brücke geschaffen, um im nächsten Kapitel auf organisationsstrukturelle bzw. -theoretische Aspekte einzugehen.

\subsubsection{Inteme Arheitsmärkte aus der Arbeitsmarktstrukturperspektive}

Wenn hier emeut eingegangen wird auf die Segmentationsansätze, so hängt dies mit der Perspektive zusammen, daB der interne Arbeitsmarkt in einer bestimmten Form segmentier ist. Segmentation wird in diesem Kontext mit einem auf die Strukturdimension bezogenen Bedeutungsinhalt belegt und unterscheidet sich damit gegenüber den von Cain aufgezählten Konzepten. Zur Charakterisierung der Strukturdimension interner Arbeitsmärkte kann also nicht auf die Segmentationsperspektive verzichtet werden.

Die Segmentationstheorien bauen auf Kerrs Ansatz auf, der zu Beginn des Kapitels kurz vorgestellt wurde. Die Segmentationstheorien reflektieren die beiden Haupttheorierichtungen, die der Neoklassik und die der Institutionalisten. Die neoklassische Denktradition geht von der Perpektive aus, daß der Gesamtarbeitsmarkt in Teilmärkte zerfällt. Diese Teilarbeimärkte funktionieren nach neoklassischer Vorstellung auf gleiche Weise. Angebot und Nachfrage werden allein gesteuert durch Lohndifferenzen. Demgegenüber steht die institutionalistisch geprägte Forschungsrichtung, die Abstand nimmt von der Prämisse, daß Teilarbeitsmärkte auf identische Weise funktionieren. Der Lohn wird hier als ein Steuerungsmechanismus anerkannt, daneben wird eine Vielfalt von anderen Organisationsmechanismen zur Angebots- und Nachfragesteuerung am Arbeitsmarkt gesehen. Mit Biehler/Brandes (1981, S. 10) lassen sich die Segmentationsansätze von der orthodoxen Arbeitsmarkttheorie abgrenzen und wie folgt zusammenfassen: 
"Erstens, Teilmärkte werden nicht mehr als stets gleichförmig funktionierend, mithin durch Lohndifferenzen gesteuert, angesehen; zweitens, die Analyse von Mobilitätsbeschränkungen zwischen den Teilmärkten wird zu ihrem zentralen Forschungsgegenstand erhoben."

Die Segmentationstheorien des Arbeitsmarktes bilden den 'Überbau' für die 'Theorie des intemen Arbeitsmarktes'. Nun soll näher eingegangen werden auf die Segmentationsansätze bzw. ihr Verhältnis zu anderen Strukturdimensionen. Bevor dieser Schritt erfolgt, sollen Arbeitsmarktstrukturebenen abgeleitet werden an Hand der von Doeringer/Piore (1971) entwickelten Theorie interner Arbeitsmärkte. Die Aufmerksamkeit gilt dabei der Arbeitsmarktstruktur, die durch wahrnehmbare Grenzen oder Hürden die Mobilität von Arbeitnehmem beschränkt. Segmentation basiert auf dem Begriff der Arbeitsmarktstrukturienung, die als "... dauerhafte, gegen kurzfristig wirksame Marktkrafte resistente, regelhafte Gestaltung des Arbeitsprozesses" (Sengenberger, 1987, S. 50) aufzufassen ist. Das Resultat dieses Prozesses ist die Arbeitsmarktstruktur.

Aus der Theorie interner Arbeitsmärkte von Doeringer/Piore (1971) lassen sich analytische Arbeitsmarktstrukturen ableiten. Es handelt sich um eine allgemeine Bestimmung der Arbeitsmarktstrukturebenen, bei der die Theorie intemer Arbeitsmärkte Pate gestanden hat.

Ziel ist es, ein möglichst konsistentes und theoriekonformes Strukturbild zu entwickeln. Bei der deduktiven Erschließung wird versucht, die einzelnen Dimensionen so zu ordnen, $\mathrm{da} B$ in der Größenordnung ein Übergang von der Gesamtheit 'Arbeitsmarkt' zu jeweils kleineren, untergeordneten Dimensionen stattindet. Dabei steht zunächst ein ganzheitliche Sichtweise des Arbeitsmarktes im Vordergrund. Mit dem extemen Arbeitsmarkt wird die Ganzheit zum Ausdruck gebracht. Als kleinste Dimension im Spektrum dieses Strukturbildes ist der einzelne Arbeitsplatz zu sehen. Zwischen diesen Dimensionen vom Arbeitsmarkt als Ganzheit bis hin zum einzelnen Arbeitsplatz als kleinste Einheit existien ein Spektrum von Strukturdimensionen (Glebbeek, 1987).

\subsubsection{Die Konkretisierung der Arbeitsmarktstruktur interner Arbeitsmärkte nach} Doeringer und Piore

Die Erschließung der Arbeitsmarktstruktur erfolgt in Form eines top-down-approach in der Art der kaskadenförmigen Stufenstruktur wie sie oben grob vorgestellt wurde. Ausgegangen wird von der Makroperspektive in Form des extemen Arbeitsmarktes (als Ganzheit) hin zu der Mikroperspektive des einzelnen Arbeitsplatzes (Element). Es geht hier um die konkrete Ausfüllung der Strukturebenen basierend auf der Theorie intemer Arbeitsmärkte von Doeringer/Piore. In der Gesamtperspektive ergibt sich aus der Rekonstruktion der Strukturdimensionen nach der Theorie des intemen Arbeitsmarktes die folgende kaskadenförmige Abbildung: 
Abb. 2: Analytische Strukturen interner Arbeitsmärkte nach Doeringer/Piore (1971)

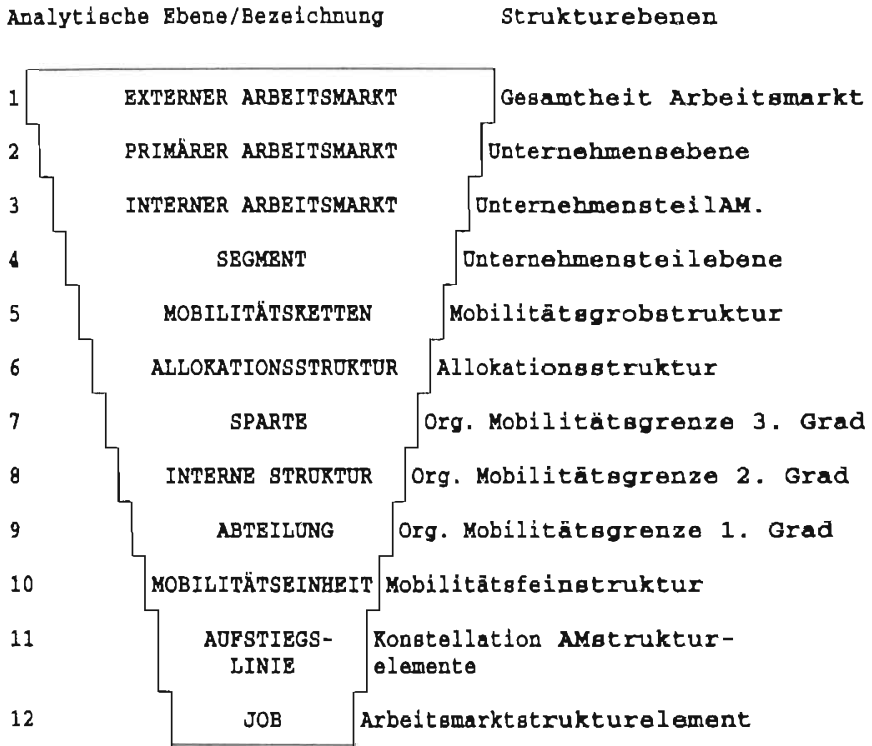

Abkürzung: Org.= Organisatorische; $\mathrm{AM}=$ Arbeitsmarkt

Diese kaskadenförmige Stuktur des Arbeitsmarktes nach Doeringer/Piore wird im folgenden besprochen. Die obige Abbildung repräsentiert die analytische Differenzierung in Strukturdimensionen.

Der exteme Arbeitsmarkt repräsentiert die Gesamtheit 'Arbeitsmarkt' als Ausgangspunkt der Betrachtung, dessen Steuerung durch ökonomische Variablen erfolgt. Vom externen Arbeitsmarkt gehen Informationsimpulse aus, die das Geschehen am intemen Arbeitsmarkt der Gesamtheit beeinflussen. Über den extemen Arbeitsmarkt kommen durch die 'ports of entry' Arbeitnehmer auf den internen Arbeitsmarkt. Der externe Arbeitsmarkt zeichnet sich als Rekrutierungsquelle und als volkswirtschaftliche Rahmenbedingung aus.

Doeringer (1967, S. 207 f.) greift zurück auf das theoretische Konstrukt 'intemer Arbeitsmarkt' von Ker, so wie es hier kurz vorgestellt wurde.

"It is governed by a set of institutional rules which delineate the boundaries of the internal market and determine its internal structure."

Die Theorie des intemen Arbeitsmarktes spricht den intemen Arbeitsmarkt als meiur ocier weniger geschlossene Einheit innerhalb des Unternehmens an. Der interne Arbeitsmarkt steht mit dem externen Arbeitsmarkt in permanentem Austausch. Doeringer/Piore haben neben dem intemen Arbeitsmarktkonzept ein weiteres Konzept, nämlich das der dualen 
Arbeitsmarkttheorie, ausgebaut und weiterentwickelt, das in einem Interdependenzverhältnis zu dem erstgenannten Konzept steht.

Aus Gründen der analytischen Transparenz soll das Konzept des dualen Arbeitsmarktes hier kurz angesprochen werden. Es geht um die Differenzierung zwischen primärem und sekundärem Arbeitsmarkt. Fragt man nach dem Verhälonis der beiden Konzepte zueinander, so bezeichnen Doeringer/Piore (1985, S. XI) den Segmentationsansatz als "...byproduct of the operation of internal labour markets." Außerdem charakterisieren Doeringer/ Piore (1971, S. 167) das Verhälmnis der Konzepte in Bezug auf den primären Arbeitsmarkt: "The primary sector consists of a series of internal markets ..." Zusammenfassend läßt sich der folgende Zusammenhang ableiten (Doeringer/Piore, 1971, S. 169):

"This view which associates primary employment with intemal labor markets, and secondary labor markets with a mixture of internal markets and jobs not belonging to intemal labor markets need not imply the strict separation of the two embodied in the concept of a dual labor market."

Primäre Arbeitsmärkte können demnach verschiedene interne Arbeitsmarktformen beinhalten. Primäre Arbeitsmärkte repräsentieren die Gesamtheit verschiedener interner Arbeitsmärkte. In der Reihenfolge des top-down-approach von Arbeitsmarktstrukturdimensionen lassen sich die folgenden Analyseebenen unterscheiden:

Analytische Arbeitsmarktstrukturebene

\section{EXTERNER ARBEITSMARKT \\ PRIMÄRER ARBEITSMARKT INTERNER ARBEITSMARKT}

Doeringer/Piore unterscheiden betriebliche (enterprise) von berufsfachlichen (craft) intermen Arbeitsmärkten. Weitere analytische Schritte sind notwendig, um von der Gesamtheit Arbeitsmarkt zu den Mikrostrukturen der betrieblichen Beschäftigung zu gelangen.

Die von Doeringer/Piore aufgestellten Arbeitsmarktkonzeple lassen sich als das Ringen um eine bessere analytische Durchdringung der Arbeitsmarktstruktur und des Arbeitsmarktgeschehen charakterisieren. Piore erweitert in einem 1975 erschienenen Artikel das duale Arbeitsmarktkonzept hinsichtlich folgender Perspektive: der primäre Arbeitsmarkt wird differenziert in ein oberes und unteres

\section{SEGMENT}

neben dem sekundären Arbeitsmarkt. Das obere Segment umfaßt Fühnungspositionen. Die Arbeitsbeziehungen bestehen aus weitgehend intemalisiertem Verhalten. Freiräume existieren hinsichtlich der Entfaltung von Initiativen und Kreativität auf der Führungsebene. Im Gegensatz dazu dominieren Vorschriften und Regeln beim unteren Segment des primären Arbeitsmarktes. 
Ein Grund für die Aufteilung in Segmente ist die theoretische Fundierung der Typologie von

\section{MOBLITÄTSKETTEN.}

Das Mobilitätskettenkonzept beinhaltet, daß Mobilität nicht zufäjlig ist, sondem in dafür festgelegten Bahnen verläuft. Arbeitsmarktstrukturen in Form von Mobilitätsketten tragen durch die Häufigkeit des Gebrauchs dazu bei, die Kosten zu minimieren.

Piore (1975) assoziiert mit dem oberen Segment des primären Arbeitsmarktes Mobilität in horizontaler und vertikaler Richtung. Die Mobilitätsketten sind zu vergleichen mit Karniereleitem (Gutteridge, 1986). Jede Verändenung der Position bedeutet gleichzeitig ein höheres Einkommen und Status (Grüner, 1989). Das untere Segment des primären Arbeitsmarktes ist nach Auffassung Piores gekennzeichnet durch Routine, Stabilität und betriebsspezifische Fähigkeiten, die vielfach durch 'automatisches Lemen' erworben wurden. Beim sekundären Segment ist die Mobilität beschränkt auf horizontale Mobilität.

Biller (1989, S.35) sieht im Mobilitätskettenkonzept den Vorteil der Erfassung von Arbeitsplatz- bzw. Tärigkeitsdifferenzienungen innerhalb der jeweiligen Segmente. Nach seiner Auffassung stellt diese analytische Dimension der Arbeitsmarktstruktur eine Modifikation des bis dahin bekannten Ansatzes dar.

Bis zum Mobiliätskettenkonzept ist noch kein Analyseniveau erreicht, das den analytischen Übergang von der Makro- zur Mikroebene des internen Arbeitsmarktes ermöglicht. Dennoch stellen Doeringer/Piore ein Instrumentarium zur Durchdringung der Mikrntbene zur Verfügung. Dies läßt sich wie folgt charakterisieren.

Die Zuweisung von Personal auf Arbeitsplätze ist die Hauptfunktion interner Arbeitsmärkte. Für beide Typen intemer Arbeitsmärkte ist die Konzeption der

\section{ALLOKATIONSSTRUKTUR}

ein entscheidender Schritt in Richtung einer analytischen Mikrofundierung der Struktur intemer Arbeitsmärkte. Die Allokationsstruknur umfaßt drei Elemente (Doeringer/Piore, 1971, S. 42 f.).

Beim ersten Element handelt es sich um den Grad der Offenheit des intemen gegenüber dem externen Arbeitsmarkt. MeBbar wird dies an Hand der Anteile von Ein- und Austrittsstellen, mit Arbeitsplatzanfordenungen und schließlich der Restriktivität der Eintrittskriterien.

Ein weiteres Element der Allokationsstruktur ist die Reichweite des internen Arbeitsmarktes. Differenziert wird zwischen Größe, geographischer Ausdehnung sowie Berufe bzw. Tätigkeiten. Als drittes Element werden die Regeln angesprochen, die die Zuordnung bzw. Verteilung von Arbeitnehmern nach Arbeitsplätzen bewirken. 
'Allokationsstruktur' ist Schlüsselbegriff für eine weitere Differenzierung der Arbeitsmarktstruktur intemer Arbeitsmärkte. Dies gilt besonders für das Element 'Reichweite des internen Arbeitsmarktes'. Inteme Arbeitsmärkte sind definiert durch ihre Grenzen. Innerhalb der Grenzen sind

\section{SPARTEN}

anzutreffen, die wiederum eine Substruktur aufweisen. Eine weitere definitorische oder konzeptionelle Bestimmung des Begriffs sucht man bei Doeringer und Piore vergebens. Die 'subdivisions' (Sparten) bilden den Rahmen für die

\section{INTERNE STRUKTUR}

eines jeden Marktes. Gedacht ist an die parallele Existenz von unterschiedlichen Teilmärkten, beispielsweise den Facharbeiter- und den betriebsspezifischen Teilmarkt. Die 'interne Struktur' bringt die Strukturperspektive innerhalb der Sparte hinsichtlich des Teilmarkts zum Ausdruck. In der Reihenfolge der Bedeutung folgt dann als analytisch kleinere 'Einheit' die

\section{ABTELUNG}

Doeringer/Piore (1971, S. 51) assoziieren mit der Existenz von Abteilungen enge 'mobility cluster', während breite 'mobility cluster' mit Arbeitsplätzen in Verbindung gebracht werden, die geringe Variation des Arbeitsinhaltes bieten. Als Beispiel werden Supermärkte und die Herstellung von elektronischen Massenprodukten angeführt. Innerhalb der Abteilung sind dann sogenannte

\section{MOBILITÄTSEINHEITEN,}

anzutreffen, die dazu beitragen sollen, den Begriff 'interne Struktur' mit Inhalt zu versehen. 'Mobility clusters' lassen sich in folgender Weise definieren. Sie sind (Doeringer/ Piore, 1971, S. 50) "... the groupings of jobs within which an employee is customarily upgraded, downgraded, transferred and laid off." Während die 'mobility clusters' die generelle Form der Kanalisierung von Mobilität charakterisieren, geht es bei (lines of progression)

\section{AUFSTIEGSLINIEN}

um die Institutionalisierung eines Lemprozesses. Dieser kann minimal aus zwei, maximal aus mehreren Arbeitsplatzkategorien bestehen. Diese Art 'Spezialcluster' vertikaler Mobilitätsrichtung trägt in hohem Maße der Spezialisierung Rechnung.

In der aktuelleren Literatur von Doeringer/Piore wird die soziale Gruppe (mit vorwiegend horizontaler Mobilitätsrichtung) genannt. Soziale Gruppen bilden in der Logik der hier entwickelten Arbeitsmarktstrukturdimension keine eigenständige Strukturdimension. Dennoch soll diese Perspektive hier kurz aufgezeigt werden. Die Erkenntnis der Einbeziehung sozialer Gruppen kommt bei Doeringer/Piore enst 1985 zum Ausdruck in einem 
'second look'. Die Autoren verbinden damit mehr Einsichten in soziale Strukturen. Sie setzen sich damit ab von neoklassischen Annahmen, die das Individuum in den Vordergrund rücken.

"... anthropological or sociological frameworks argue that purposive human activity can only take place within a social context. Human activity requires a framework for thought, goal setting and communication, and such frameworks are preeminently social." (Doeringer(Piore, 1985, S. XXIV)

Mit dieser Perspektive bauen die Autoren eine Brücke zur Kultur bzw. Subkultur, die sich in Gruppen herauskristallisiert und die damit eine entscheidende Größe für die Allokation auf dem intemen Arbeitsmarkt wird.

Die kleinste Einheit innerhalb des Spektrums der Arbeitsmarktstrukturebenen ist schließlich der einzelne Arbeitsplatz,

JOB.

Auch idiosynkratische Arbeitsplätze sind in Strukturen, so wie sie hier beschrieben wurden, eingebunden (Miner, 1987). Es handelt sich um Arbeitsplätze, bei denen die Persönlichkeit des Inhabers die Aufgabeninhalte selbständig ausfüllt, ohne daß eine Aufgabenbeschreibung und Weisungsgebundenheit des Stelleninhabers vorliegt.

Die deutliche Strukturierung des internen Arbeitsmarktes ist Mittel zur Durchsetzung allokativer Effizienz. Die beiden Autoren machen deutlich, daB inteme Strukturierung der Förderung von Effizienz dient, indem Ausbildungs- und Fluktuationskosten reduziert werden. Hier artikuliert sich ein Interesse des Managements. Gleichzeitig betonen die Autoren, daß eine effiziente Arbeitsmarktstruktur vielfach Ausdruck eines Kompromisses mit den Interessen der Arbeitskräfte ist, die Beschäftigungssicherheit und Fortkommen als Zielvorstellung artikulieren.

Die analytische Erschließung der Determinanten der Arbeitsmarktstruktur des internen Arbeitsmarktes zeigt das AusmaB der Komplexität, das in Form der Theorie des intemen Arbeitsmarktes zu integrieren versucht wurde. Die Ableitung der einzelnen Strukturdeterminanten bedeutet nicht, daß damit ein eindeutiges Konzept zur empirischen Überprüfung intermer Arbeitsmärkte gegeben ist. Defizite in Form logischer Brüche bestehen. Sie kommen insbesondere zum Ausdruck bei der Ausfüllung der Segmente durch jeweils kleinere 'Einheiten' bzw. Strukturdeterminanten. Die jeweiligen Strukturen, die im topdown-approach aufgezeigt wurden, ergänzen sich mehr oder weniger. Mit der Arbeit von Doeringer/Piore liegl jedoch der weitestgehende Ansatz zur analytischen Durchdringung internẹr Arbeitsmärkte vor.

Die Arbeitsmarktstrukturdimensionen sind das Resultat einer stets veränderten Betrachtungsweise. Daher erklärt sich auch, daß die hier aufgezeigte Ausfüllung der Strukturen keinem 'nahtlosen' Konzept entspricht. In der dualen Arbeitsmarkttheorie wird der Arbeitsmarkt als Ganzheit betrachtet, die in zwei (oder mehrere) Teile zerfällt. Die weitestgehende Konkretisierung der Struktur bezog sich auf Mobilitätsketten innerhalb von 
Unternehmen. Hier müßre sich auf der Allokationsstrukturebene logischerweise die nächst kleiner dimensionierte Struktur in Form der 'mobility cluster' fortsetzen. Stattdessen bemühen Doeringer/Piore eine 'eigenwillige' Strukrureinteilung, die aus der Allokationsstruktur, den 'subdivisions', der internen Struktur und schließlich der Abteilung besteht. Diese Perspektive entspricht dem Interesse, die Mikrofundierung intemer Arbeitsmarktstrukturen zu erschließen.

Die logischen Brüche, die sich in der Uneinheitlichkeit der Strukhurebenen artikulieren, werden deutlich, wenn man nach der Herkunft der einzelnen Dimensionen fragt. 'Subdivisions' und 'departments' sind Einteilungkriterien aus der Organisationstheorie. Mobilitätsketten und mobility cluster sind Kriterien, die der Arbeitsmarktstrukturtheorie entstammen. Bei sozialen Gruppen stehen Menschen statt Organisationsstrukturen im Vordergrund.

Als Schlußfolgerung läßt sich konstatieren, daß die Strukturierung interner Arbeitsmärkte durch Doeringer/Piore nach uneinheitlichen Kriterien erfolgt. Die Ableitung eines in sich konsistenten Strukturbildes kann nur annähemd erreicht werden. Von einem solchen Strukturbild wäre zu erwarten, daß Aufschluß erfolgt über die jeweiligen Grenzen (boundaries) und deren weitere Differenzierung nach einem einheitlichen logisch konsistenten Maßstab. Dennoch bietet die Konzeption Doeninger/Piores trotz der benannten Schwächen ein Ausgangskonzept. Um diese Perspektive zu erhärten, soll vergleichend auf die Konzeption von Lutz und Sengenberger eingangen werden.

2.2.4 Die Arbeitsmarktstruktur intemer Arbeitsmärkte in der Konzeption von Lutz und Sengenberger

Neben Doeringer/Piore haben sich Lutr und Sengenberger insbesondere mit betriebsinternen Arbeitsmärkten auseinandergesetzt. Zwei Gründe sind für deren Auswahl maßgebend. Zum einen die spezifische europäische Sichtweise, zum anderen der deutlichere zeitliche Bezug zur Gegenwart. Aufgrund dessen, daß Lutz und Sengenberger in der Vergangenheit in hohem Maße kooperiert haben, ergeben sich bei den eigenständigen Konzepten der Autoren deutliche Übereinstimmungen bzw. Abweichungen in Nuancen. In diesem Abschnitt wird deshalb die Konzeption des jeweiligen Autors kontextbezogen vorgestellt.

Die bei Doeringer/Piore aufgezeigten Konzeptionen des internen Arbeitsmarktes und der dualen Arbeitsmarkttheorie sind auch bei Lutz (1987) zentraler Gegenstand. Lutz versucht in seinem Ansatz die beiden Konzepte zu integrieren. Ausgangspunkt ist die betriebliche Arbeitskräftestrategie, die die Entstehung von internen Arbeitsmärkten begünstigt. Sie ist so breit definiert, daß sie sich unter dem Begriff, "...betriebliche Strukturen, Politiken und Maßnahmen..." (Lutz, 1987, S. 20) zusammenfassen lassen.

Die Motive der Entstehung intemer Arbeitsmärkte liegen im Interesse an der langfristigen Nutzung der Arbeitskraft und der Stabilität der Arbeitsstrukturen und der Vorteilhaftigkeit. Dazu gehört auch "... eine besondere Form des Zugriffs zum externen Arbeitsmarkt und der dort bestehenden Konkurrenz zwischen Anbietem und Arbeitskraft,..." (Lutz, 1987, S. 14). 
Im folgenden sind die Arbeitsmarktstrukturdimensionen von Lutz und Sengenberger zusammengefaBt. Im einzelnen sollen sie auch hier kurz vorgestellt werden, bevor sie im Rahmen eines Vergleich mit denen von Doeringer/Piore einander gegenüber gestellt werden. Auch in diesem Abschnitt soll die Herausarbeitung der von Lutz hantierten Arbeitsmarktstrukturdimensionen in der Reihenfolge von 'global nach spezifisch' erfolgen. Der

\section{EXTERNE ARBEITSMARKT}

ist nach Lutz ebenfalls Gegenstand der Arbeitskräftestrategie wie oben kurz angedeutet wurde, d.h. vom internen Arbeitsmarkt gehen Impulse zur Beeinflussung des externen Arbeitsmarktes aus. Daneben ist die Form des Austauschs mit dem intemen Arbeitsmarkt langfristig angelegt. Ebenfalls geht vom extemen Arbeitsmarkt der Druck von Beschäftigungsrisiko und Arbeitsmarktkonkurrenz aus. Hierdurch werden auf dem internen Arbeitsmarkt spezifische Verhaltensweisen entwickelt.

Sengenberger geht von einem

\section{GESAMTARBEITSMARKT}

aus, der sich aufteilt in

\section{TELLARBEITSMÄRKTE.}

Der Gesamtarbeitsmarkt läßt sich in 'berufsfachliche Märkte' und 'betriebsinterne Märkte' sowie in zwei Formen 'externer Märkte' aufteilen. Die externen Märkte erfahren die Differenzierung in 'unabhängige externe Märkte' (Jedermannsmärkte) und 'abhängige externe Märkte' (Puffermärkte).

Die folgende Arbeitsmarktstrukturdimension ist nach Lutz die des

\section{BETRIEBLICHEN ARBEITSSYSTEMS.}

Hier wird die Strukturebene angesprochen, bei der sich "...betriebliche Arbeitskräftestrategien artikulieren und manifestieren ... in Strukturen relativ hoher Stabilität und innerer Geschlossenheit..." (Lutz, 1987, S. 20).

Als sogenannte 'Grunddimensionen' von Arbeitskräftesystemen unterscheidet Lutz Binnenund Außenverhältnis sowie den Zeithorizont der Vermittlung von Binnen- und Außenanforderungen. Eine weitere Vertiefung der betrieblichen Arbeitssysteme ist die idealtypische Klassifizierung in 'interner Arbeitsmarkt' und 'Facharbeiterbetrieb' (professionelle Arbeitsorganisation) sowie 'tayloristische Arbeitsorganisation', Beim Arbeitssystem 'Facharbeiterbetrieb' steht die 'benfffachliche' Qualifikation der Arbeitnehmer im Vordergrund, die abhängig ist von betriebsextern vorgegebenen Bildungsnormen. Kennzeichen dieses Arbeitssystems sind die geringe Arbeitsteilung, eine tlache Arbeitsplatzhierarchie und betriebsübergreifende Mobilitätsmuster. Alle drei Formen können Ausprägungen eines unternehmensinternen Arbeitsmarktes sein. 
Das Arbeitssystem vom Typ 'taylorischer Arbeitsorganisation' ist auf das 'Binnenverhältnis' gerichtet und stark arbeitsteilig. Permanente Kontrolle ist ein entscheidendes Kennzeichen dieses Arbeitssystems. Anwendung findet dieses Arbeitssystem in der Massenproduktion.

Lutz weist darauf hin, daß in der Realität selten die Idealformen, meistens die Mischformen vorkommen. Dem internen Arbeitsmarkt kommt dabei eine besondere Bedeutung zu.

Auf dieser Ebene vollzieht Lutz die Integration der Konzepte 'intemer Arbeitsmarkt' und 'betriebszentrierte Arbeitsmarktsegmentation'. Der

\section{INTERNE ARBEITSMARKT}

basiert als umfassenderer Begriff auf drei von insgesamt fünf definierten Merkmalen betriebszentrierter Arbeitsmarktsegmentation. Diese beruhen auf 'ports of entry' für neue Arbeitnehmer, einer arbeitsplatzbezogenen Qualifikations-, Verantwortungs- und Verdienstabstufung sowie auf hierarchicbezogene Mobilitätsmuster. Betriebszentrierte Arbeitsmarktsegmentation ist dann gegeben, wenn statt der beim intemen Arbeitsmarkt gegebenen Betriebsbindung eine wechselseitige Bindung zwischen Betrieb und Beschäftigten entsteht. Außerdem muß das Allokationmuster gegenüber den Beschäftigten ein hohes $\mathrm{MaB}$ an Verbindlichkeit aufweisen.

Filtert man die Essenz aus dem Versuch, zwei Konzepte zu integrieren, so wird über die betriebliche Arbeitsmarktsegmentation ein spezifischerer interner Arbeitsmarkt angesprochen, auf dem ein höheres $\mathrm{Maß}$ an Internalisierung und Organisationskultur die Beziehung zwischen Arbeitnehmern und Arbeitgebem bestimmt. Vergebens sucht man in diesem Kontext nach der strukturellen Konsequenz in Form von Segmentationslinien, die durch wie auch immer gestaltete Segmentgrenzen, die Arbeitnehmermobilität einschränken. Eine deutlich gegebene Arbeitsmarktstruktur, festzustellen an Hand von Segmenten, läßt sich in Bezug auf das Lutz-Konzept der betrieblichen Arbeitsmarktsegmentation nicht ableiten.

Vom

\section{INTERNEN ARBEITSMARKT}

kann man nach Sengenberger sprechen, in dem (Sengenberger, 1987, S.150)

"a) auf Arheitskraft bezogene Anpassungsvorgänge untemehmens- bzw. betriebsintem, $d$. h. ohne dirckten Rückgriff aul exteme Arbeitsmärkte, vollzogen werden,

b) den im Untemehmen oder Betrieb beschäftigten Arbeitskräften eine Vorzugsbehandlung vor Außenstehenden zuteil wird. Diese Präferierung der 'ins' gegenüber den 'outs' spiegelt sich in der personalpolitischen Praxis der Betriebe durch Maxime wie 'Umstellung vor Einstellung' und 'Aufstieg vor Einstieg' wider."

Die hier vorgestellte Definition ist geprägt von der Interaktion zwischen in- und extemem Arbeitsmarkt. Sic unterscheidet sich damit nicht von der Auffassung Lutz. Ähnlich wie 
Lutz kennzeichnet Sengenberger mit 'betriebszentrierter Arbeitsmarktsegmentation' den höheren Grad an Geschlossenheit interner Arbeitsmärkte.

Eine weitere Arbeitsmarktstrukturdimension bei Lutz knüpft an die oben vorgenommene Differenzierung der sogenannten 'Grunddimensionen' an. Im Rahmen des 'Binnenverhältnisses' werden drei 'zentrale Kategorien': die betriebliche Leistungspolitik, der Arbeitseinsatz und die

\section{ARBEITSORGANISATION}

unterschieden. Hierbei werden die aus der Produktion gestellten Anforderungen zu 'Leistungspaketen' zusammengestellt und in Form von Arbeitsplätzen gebündelt. Als 'Strukturelement bzw. -moment' (Lutz, 1987, S. 53) oder an anderer Stelle (S. 54) als 'zentrale Dimension' betrieblicher Arbeitsmärkte (hier für den Typ 'interner Arbeitsmarkt') erfährt die Arbeitsorganisation eine Akzentuierung. Die Arbeitsorganisation wird aufgefaßt als globale Struktur zur Erzeugung von 'Qualifikation'.

Mit der 'Dimension intemer Arbeitsmärkte' zeigt Sengenberger den Reichtum an Formen und Funktionsweisen intemer Arbeitsmärkte auf. Die weitere Differenzienung der 'Dimension' führt zu zwei 'Grunddimensionen', wovon die eine die vertikale (hierarchische) Dimension darstellt, die andere die 'horizontale (nicht-hierarchische) Dimension'.

Die nächstfolgende von Lutz benutzte 'kleinere' Arbeitsmarktstrukturdimension ist die der

\section{MOBLITÄTSKETTE,}

die aus mehreren untereinander verbundenen Arbeitsplätzen besteht und einher geht mit einem Qualifikationsprozeß der Arbeitnehmer. Die Länge der Mobilitätskette ist abhängig von der Zielsetzung der beabsichtigten Qualifizierung.

Ein weitere Konkretisierung (und Eingrenzung) der vertikalen Dimension nimmt Sengenberger in der Form der betrieblichen

\section{HIERARCHIE VON ARBEITSPLÄTZEN.}

vor. Die nächst kleinere Dimension nach Lutz besteht aus der

\section{ARBEITSPLATZSTRUKTUR.}

Damit soll dem Grad der vertikalen Arbeitsteilung Rechnung getragen werden. Äquivalent bei Sengenberger sind

\section{ARBEITSPLATZKETTEN.}

Schließlich stimmen Lutz und Sengenberger bei der kleinsten Dimension des

\section{ARBEITSPLATZES}


überein. Auf diesem Niveau wirken Mobilitätsimpulse ein, die Lutz zurückführ auf den dernographischen Prozeß und die Differenzierung von materiellen und immateriellen Gratifikationen.

Betrachtet man den Ansatz von Lutz und vergleicht ihn mit den Arbeitsmarktstrukturdimensionen von Doeringer/Piore, so sind Parallelen, aber auch deutliche Unterschiede aufzuzeigen. Bei Doeringer/Piore dominiert die Arbeitsmarktstrukturperspektive, bei Lutz die Arbeitskräftestrategie, die ihren Ausdruck findet im Arbeitssystem. Das Arbeitssystem läßt sich weiter differenzieren bis hin zu den Arbeitsmarktstrukturelementen. Jedoch wird diese Erwartung bei Lutz und Sengenberger nicht erfüllt. Dies gilt ebenso für die Konkretisierung der betrieblichen Arbeitsmarktsegmentation in Form deutlich abgrenzbarer Segmentstrukturen. Vergleicht man die analytischen Arbeitsmarktsmarktstrukturen in Form der folgenden Übersicht, dann fallen die Unterschiede der jeweiligen Konzepte deutlich ins Auge.

\section{Abb. 3: Analytische Arbeitsmarktstrukturen im Vergleich}

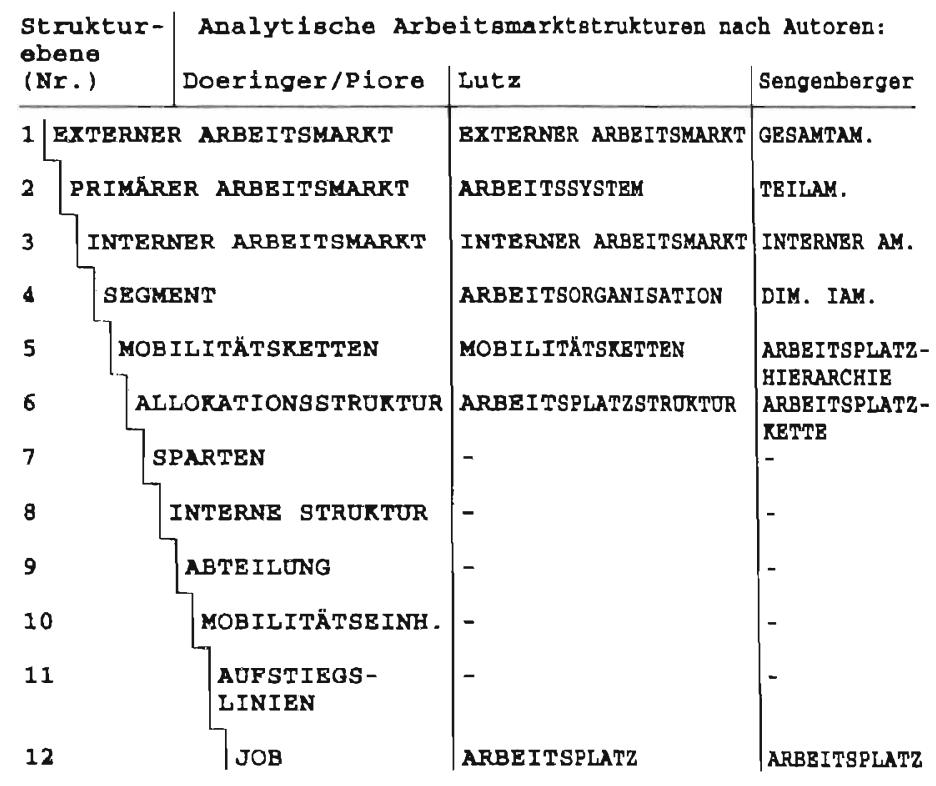

AM=Arbeitsmarkt; LAM=Interner Arbeitsmarkt; Dim.=Dimension

Beim Vergleich der drei Konzepte fällt auf, daß die Konzeption von Doeringer/Piore deutlich differenzierter ist als die der beiden anderen Autoren. Nach Doeringer/Piore lassen sich insgesamt 12 Arbeitsmarktstrukturebenen ableiten im Gegensatz zu den jeweils 7 Ebenen bei Lutz und Sengenberger. Die beiden letztgenannten Autoren berücksichtigen nur einen Teil der von Doeringer/Piore erschlossenen mikroorganisatorischen Mobilitätsgrenzen bzw. -strukturen. Die analytische Erschließung der Arbeitsmarktstrukturebenen 
bildet bei der Konzeptionalisienung von innerorganisatorischen Mobilitätsgrenzen eine bedeutende Rolle. In Kapitel 3 wird darauf eingegangen.

\subsection{Schlußfolgerungen}

Die Auseinandersetzung mit neoklassischer und institutioneller Arbeitsmarkttheorie deutet eine Präferenz zu Gunsten der institutionellen Arbeitsmarkttheorie an. Dies ergibt sich aus dem Gegenstand und der Zielsetzung der Untersuchung sowie der analytischen ErschlicBung der Komplexität der Zusammenhänge, die den internen Arbeitsmarkt ausmachen. Die institutionelle Arbeitsmarkttheorie bietet die Perspektive der 'Kompatibilität' zu den organisationstheoretischen Ansätzen, auf die im nächsten Kapitel näher eingegangen werden soll.

Die analytische Durchdringung der Arbeitsmarktstruktur hat unter den verschiedenen Autoren Übereinstimmungen aber auch Abweichungen aufgezeigt. Weitestgehend von der analytischen Durchdringung ist der Ansatz von Doeringer/Piore. Deutlich zeigt sich, daß die Identifizierung der Strukturebenen auf den verschiedensten Niveaus umfangreicher ist als in der einschlägigen Literatur bislang behandelt. Die Arbeitsmarktstrukturdimensionen wie sie hier analytisch aufgezeigt wurden, haben Einfluß auf die Allokation von Arbeit. Definitorischer Ausgangspunkt für die weitere Betrachtung ist zunächst die Perspektive von Doeringer und Piore (1971). Welche Bedeutung im einzelnen ausgeht, läßt sich nach Einbeziehung der organisationspezifischen Betrachtung beurteilen. Festzuhalten bleibt, daß bei der Erschließung und Einteilung der allokationswirksamen Grenzen interner Arbeitsmärkte zunächst die Außengrenze interner Arbeitsmärkte eine Rolle spielt.

Darüberhinaus konzentriert sich die Aufmerksumkeit auf eine Einteilung des intemen Arbeitsmarktes in Segmenten, Mobilitätsketten, Abteilungen sowie Arbeitsplatzanordnungen. In diesen Aspekten zeichnet sich eine weitgehende Übereinstimmung der unterschiedlichen Ausgangsperspektive ab.

In diesem Kapitel wurde von Arbeitsmarkttheorien hinsichtlich betrieblicher Beschäftigung ausgegangen. Erschlossen wurden Arbeitsmarktstrukturdimensionen, die ein erweitertes Verständnis ermöglichen sollen über bestimmte betriebliche Arbeitsmarktformen. Unter Berücksichtigung der Organisationsperspektive in Kapitel 3 werden die erwähnten Arbeitsmarktstrukturdimensionen konzeptionell verarbeitet. Sie erscheinen in Kapitel 7 und 8 bei der Einschätzung des Einflusses von Organisationsgrenzen auf die Allokation wieder.

An dieser Stelle ergibt sich die Frage, wie von einem organisationstheoretischen Standpunkt Ansätze zur konzeptionellen Erschließung untemehmensintemer Arbeitsmärkte aussehen (Kalleberg/Berg, 1987). Wo liegen dann die Nuancen im Vergleich zu den arbeitsmarkttheoretischen Ansäızen? Dieser Fragestellung gilt es im fulgenden Kapitel nachzugehen. 
"... there is reason to be optimistic that a 'new science of organization' will take shape during the decade of the 1990s..."

Williamson, 1991, S. 294

\section{Organisationstheoretische Erklärung interner Arbeitsmärkte}

Während im vorhergehenden Kapitel die Arbeitsmarktheorieperspektive behandelt wurde, gilt es in diesem Kapitel die Aufmerksamkeit auf die organisationstheoretische Betrachtung interner Arbeitsmärkte zu richten. Ziel ist es, die Arbeitmarkt- und Organisationsperspektive zusammen zu führen. Dieses Kapitel thematisiert diese Problematik über mehrere Abschnitte. Zunächst wird es um das Verhälmis von Arbeitsmarkt- und Organisationstheorie (Pfeffer, 1982) gehen und der Frage, warum bis heute beide Theorieströmungen sich nur wenig gegenseitig befruchtet haben, obwohl ein großer Vorrat an Gemeinsamkeilen (Albeda, 1985) besteht. Ein weiterer Abschnitt befaßt sich mit dem Organisationsrahmenkonzept zur Erschließung der für den intemen Arbeitsmarkt relevanten Organisationsstrukturen und Funktionselemente. Ein weiterer Abschnitt ist den konstituierenden Determinanten interner Arbeitsmärkte gewidmet.

Danach stehen zwei Organisationstheorien im Mittelpunkt, die Transaktionskostentheorie und die Kontingenztheorie, Über sie soll das organisationstheoretische Erklärungspotential intemer Arbeitsmärkte erschlossen bzw. auf Kompatibilität geprüft werden.

Ziel ist es, über die Organisationstheorie eine Konzeption organisationsbezogener Beschäftigungskennzeichen zu erarbeiten.

\subsection{Ausgangspunkte einer organisationstheoretischen Erklärung interner Arbeitsmärkte}

\subsubsection{Arbeitsmarkt- und Organisationstheorie, zwei kompatible Theorieströme?}

Beide Theorien haben sich in der Vergangenheit unverbunden gegenübergestanden. Die Konvergenz des Anliegens und der Theorieinhalte haben noch nicht zu einer weiteren Durchdringung bzw. der Findung integrativer Elemente beider Theorien geführt. Erste Ansätze zur Einleitung einer integrierten Forschungsperspektive sind zu konstatieren (Braverman, 1977). Ostermann (1982. 1987) betont als Vertreter mit einer arbeitsmarkttheoretischen Argumentationsrichtung die Notwendigkeit, die 'Struktur der Firma' über die Organisationsstruktur und deren Determinanten zu erschließen. Noch stärker vom 
Integrationsgedanken beseelt, setzen Spilerman (1977) und Stewmann/Konda (1983) in einer Untersuchung über 'organisatorische Arbeitsmärkte' eine interdisziplinäre Vorgehensweise um.

Sucht man nach Gründen, die bislang einer integrativen Forschungsweise entgegenstehen, dann liegt es vielfach an den Grenzen, die die jeweiligen Fachgebiete umgeben. Dies hat dazu geführt, daß die Arbeitsmarkttheoric (überwiegend neoklassisch geprägt) lange Zeit die Mikroorganisation als Forschungsgegenstand ausgeklammert hat. Umgekehrt scheinen unter organisationstheoretischer Perspektive Arbeitsmarktprobleme nicht zum zentralen Erkenntnisinteresse zu gehören. Erst in der jüngeren Vergangenheit zeigen Organisationstheorien (Kieser/Kubicek, 1978) (Transaktionskostentheorie und Kontingenztheorie) mehr die Anlage zur einer fachübergreifenden Sichtweise (Michaelis, 1985). Auch im Rahmen nationaler Forschungskultur sind deutliche Unterschiede bei der gegenseitigen Befruchtung der beiden Theorieansätze festzustellen. So sind die Vereinigten Staaten Trendsetter in Bezug auf integrative Forschungsvorhaben (Kieser, 1981). Erst mit zeitlicher Verzögerung sind Anzeichen einer entstehenden Forschungskultur in Europa wahrzunehmen.

Die Untersuchung von Ostermann (1988) bietet ein Integrationsbeispiel nicht nur hinsichtlich der obigen Perspektive, sondem darüberhinaus kontinentübergreifend. Festzuhalten bleibt, daß Arbeitsmarkt- bzw. Organisationstheorie kompatible Theorieströmungen in Bezug auf das Erkenntnisobjekt dieser Studie bilden.

\subsubsection{Das Organisationsrahmenkonzept zur Erschließung interner Arbeitsmärkte}

Ähnlich dem Konzept Kerrs für die Arbeitsmarkttheorien soll an dieser Stelle das Fundament gelegt werden für eine organisationstheoretische Erschließung interner Arbeitsmärkte (Zucker, 1987). Als 'Überbau', der die Bezeichnung 'Organisationsrahmenkonzept' trägt, wird hier ein Konzept vorgestellt, das den 'Rahmen' bildet für die organisationstheoretische Fundierung. Ziel ist es, das Erkenntnisinteresse zu strukturieren, bevor die organisationstheoretische Ausfüllung erfolgt (Cappelli/Cascio, 1991).

Das Organisationsrahmenkonzept läßt sich definieren als Allokationsnetzwerk betrieblicher Beschäftigung. Dieses besteht aus zwei Elementen. Zum einen geht es um die Grenzen von Organisationseinheiten, z. B. Abteilung, die die Allokation beeinflussen. Zum anderen geht es um die Struktur innerhalb dieser Organisationseinheiten, z. B. die Funktionskette.

Das Organisationsrahmenkonzept zielt auf die Konkretisierung der betrieblichen Arbeitsmärkte. Neben den innerorganisatorischen Mobilitätsgrenzen, damit wird der Bezug zu den Arbeitsmarktstrukturdimensionen (Kapitel 2) hergestellt, gilt es, innerhalb der Einheiten die jeweilige Position des Beschäftigten zu lokalisieren bzw. die organisatorischen Grenzen, die den Beschäftigten umgeben. Es liegt in der Natur eines 'Organisationsrahmenkonzeptes', das dies ergänzungsbedürftig ist. Der 'Rahmen' des Konzeptes wird ausgefüllt durch spezifische Sichtweise der Transaktionskostentheorie und der Kontingenztheorie im Rahmen dieses Kapitels.

Um nicht die Organisationsidentität aufzugeben, bilden Organisationen gegenüber der Umwelt Grenzen. Diese können unterschiedliche Formen annehmen. Bei der Vielfalt der 
Möglichkeiten der Gestaltung und Steuerung der Organisationsgrenzen entstehen unterschiedliche Durchlässigkeitsgrade. In Verbindung mit intemen Arbeitsmärkten interessieren vor allem die sozialen bzw, strukturellen Grenzen (social boundaries) von Organisationen (Vietorisz/Harrison, 1978). Die im folgenden entwickelten konzeptionellen Vorstellungen gehen auf Scott zurück. Scotts Ideen (1987, S. 171) basieren auf einem Konzept unter Einbeziehung der oben genannten Elemente in Bezug auf Organisationsstruktur (Warmerdam/van den Berg, 1986) bzw. -grenze.

"A collectivity is a specific instance of social organization - an indentifiable 'chunk' of the social order. ...the criteria for the existence of a collectivity are (1) a delimited social structure - that is, a bounded network of social relations - and (2) a normative order applicable to the participants linked by the network. All collectivities - including informal groups, communities, organizations, and entire societies - possess, by definition, boundaries that distinguish them from other systems."

Mit dem Entstehen interner Arbeitsmärkte stellt sich das Problem des Steuems sozialer Grenzen der Organisation sowohl nach außen als auch nach innen. Scott betont, daß mit der Zunahme der Zahl intemer Arbeitsmärkte der Mobilitätsprozeß innerhalb der Gesellschaft durch organisatorische Arbeitsplatzstrukturen einschließlich der Kontrolle (Watts, 1981) beherrscht wird.

"Jobs are locations within organizational structures, and so if we wish to understand how individuals are matched with jobs, we need to know what positions exist, how they are connected, and what rules are employed for sorting workers among them. This kind of research can be conducted only at the organizational level." (Scott, 1987, S. 179)

Konkreter ausgedrückt bedeutet (Scott, 1987, S. 180) dies: "...we know far too little about the intraorganizational processes by which employees, once hired, are routed to different organizational locations that, independent of individuals' abilities, have a major impact on potential earnings and career opportunities."

Scott weist hier auf das dynamische Moment innerhalb der jeweiligen Organisationseinheiten hin, angesprochen werden hier Mobilitätsprozesse. Die sozialen Organisationsgrenzen (Endruweit, 1988) determinieren nicht nur den Zutritt zum internen Arbeitsmarkt sondem auch die sozialen Grenzen innerhalb der Organisation. Diese besitzen einen unterschiedlichen Grad an Permeabilität (Zucker, 1988). So wie an anderer Stelle angedeutet, soll hier die intraorganisationale Mobilität (White/Althauser, 1984) von Arbeitnehmem näher betrachtet sowie die jeweilige Konzeption innerorganisatorischer Grenzen als potentielle Beschränkungen der Mobilität.

Das Organisationsrahmenkonzept ergänzt die im vorigen Kapitel entwickelte Arbeitsmarktstrukturperspektive. Das Organisationsrahmenkonzept bildet eine 'Dachkonstruktion', die der Ergänzung und Ausfüllung durch die jeweilige Organisationstheorie bedarf. Bevor darauf im einzelnen in diesem Kapitel eingegangen wird, gilt es zu fragen, worin bestehen die Kennzeichen und Determinanten der Organisation des intemen Arbeitsmarktes? 


\subsection{Kennzeichen der Organisation des internen Arbeitsmarktes}

In diesem Abschnitt sollen die Kennzeichen der Organisation des internen Arbeitsmarktes angesprochen werden. Daneben wird eingangen auf Determinanten der Entstehung interner Arbeitsmärkte. Dabei werden sowohl theoretische Überlegungen als auch empirische Befunde in die Analyse einbezogen.

Den Ausgangspunkt bilden Fragen, die auf das Zustandekommen des internen Arbeitsmarktes zielen. Warum haben einige Organisationen im Gegensatz zu anderen die Neigung, interne Arbeitsmärkte zu entwickeln? Unter welchen Bedingungen entscheiden Manager, ihre Organisation mit einem intemen Arbeitsmarkt auszustatten, anstatt auf Marktkräfte zu vertrauen? Welchen Stellenwert nimmt der interne Arbeitsmarkt innerhalb der Organisation ein? Bleibt er auf ein Subsystem innerhalb des Unternehmens beschränikt oder umfaßt er alle möglichen Formen der Beschäftigung? Wie verändert sich die Beschäftigungsentwicklung bei intemen Arbeitsmärkten im Zeitablauf? Dies sind einige Fragen, auf die im weiteren Verlauf der Untersuchung versucht wird, Teilantworten zu geben.

\subsubsection{Interne Arbeitmärkte als ökonomische Antwort}

Die Auffassung, daß interne Arbeitsmärkte anpassungsfähige organisatorische und ökonomische Lösungen darstellen in Bezug auf eine sich permanent ändernde Organisationsumgebung, (Pfeffer/Cohen, 1984; Bills, 1987) kennzeichnet die Literatur. Interne Arbeitsmärkte entstehen als Folge der Anpassung des Beschäftigungssystems an Veränderungen in der Umgebung (Sengenberger, 1987). Auf dieser Ebene besteht unter den Arbeitsmarktund Organisationsforschem unterschiedlicher Theoriestömungen ein breiter Konsens. Die unterschiedlichen Theorieströmungen kommen bei den Details des Funktionierens interner Arbeitsmärkte zum Vorschein. Diese werden im Laufe dieses Abschnittes problematisiert.

Die Kennzeichen interner Arbeitsmärkte, die in der Literatur diskutiert werden, sind Annahrnen und damit theoretischer Natur. Die Kennzeichen sollen hier von MeBindikatoren des internen Arbeitsmarktes unterschieden werden, die Gegenstand eines weiteren Abschnitts sind. Ein Teil der Kennzeichen konnte im Rahmen empirischer Untersuchungen falsifiziert oder verifiziert werden. Van Bergeijk/de Grip (1986) unterscheiden zwei Gesichtspunkte, unter denen die Kennzeichen interner Arbeitsmärkte gesehen werden können. Dabei handelt es sich um intrinsische und extrinsische Kennzeichen. Erstere problematisieren das Organisationsgeschehen auf intermen Arbeitsmärkten, die andere Perspektive rückt die Austauschbeziehung mit dem externen Arbeitsmarkt bzw. die Organisationsumgebung in den Mittelpunkt. Als solche werden hier angesprochen: die externen ökonomischen Bedingungen, die Rolle der Gewerkschaften, als intrinsische die organisatorischen Kennzeichen interner Arbeitsmärkte sowie die theoriebezogenen Gründe der Entstehung intemer Arbeitsmärkte. Die folgende Übersicht in- und extrinsischer Faktoren ist nicht systematisch sondem exemplarisch. 
Abb. 4: Intrinsische Kennzeichen und extrinsische Determinanten der Organisation interner Arbeitsmärkte

INTRINSISCHE KBNNZEICHEN INTERNBR ARBEITSMÂRKTB

\begin{tabular}{|c|c|c|}
\hline $\begin{array}{l}\text { Geschlossenhelt } \\
\text { durch ports of entry }\end{array}$ & Mobilitätoketten & $\begin{array}{l}\text { Institutionalisie- } \\
\text { rung von Gewohnheit }\end{array}$ \\
\hline Perbonalabtetlung & $\begin{array}{l}\text { Betriebsopezifische } \\
\text { Qualifikation }\end{array}$ & Learning on the job \\
\hline Beförderung & $\begin{array}{l}\text { Lange Beschäfti- } \\
\text { gungedauer }\end{array}$ & $\begin{array}{l}\text { Demographie } \\
\text { Personal }\end{array}$ \\
\hline $\begin{array}{l}\text { Koppelung } \\
\text { Job-Gehalt }\end{array}$ & $\begin{array}{l}\text { Organisations- } \\
\text { kultur }\end{array}$ & \begin{tabular}{|l} 
Pluktuation \\
(Mobilität)
\end{tabular} \\
\hline
\end{tabular}

\section{EXTRINSISCHE DETERMINANTEN INTERNER ARBEITSKÄRRTE}

\begin{tabular}{l|l|l}
$\begin{array}{l}\text { Ookonomische Rahmen- } \\
\text { Bedingungen }\end{array}$ & Gewerkschaften & $\begin{array}{l}\text { Wirtschaftliches } \\
\text { Wachstum }\end{array}$ \\
\hline Sektor & Fluktuation & Konjunktur
\end{tabular}

Im folgenden soll kurz eingegangen werden auf die verschiedenen Kennzeichen interner Arbeitsmärkte.

\subsubsection{Exteme ökonomische Bedingungen als Determinante intemer Arbeitsmärkte}

Zunächst sind interne Arbeitsmärkte Ausdruck externer ökonomischer Bedingungen. Allgemein läßt sich sagen, daß ein angespannter Arbeitsmarkt in Phasen der Hochkonjunktur oder allgemeinen Wachstums (Paauwe, 1989; Gunz, 1988) aber auch durch die ökonomischen Rahmenbedingungen die Entwicklung von intemen Arbeitsmärkten erleichtert. Ein Mangel an qualifizierten extemen Kandidaten führt unter diesen Bedingungen zu Schwierigkeiten in der Rekrutierung geeigneter Personen. Dies kann die Einrichtung intemer Arbeitsmärktc fördem (Elbaum, 1983; Bills, 1987). Ziel dabei ist es, die Arbeitnehmer durch finanzielle Anreize und 'Stansgewinn' an das Unternehmen zu binden.

Fluktuation repräsentiert eine ambivalente Erscheinung in Bezug auf den intemen Arbeitsmarkt. Fluknuation kann sowohl bei den intrinsischen als auch bei der extrinsischen Faktoren eine Rolle spielen. Die Fluktuationsrate wird als ad hoc-Kriterium bzw. als Maß für den Personalaustausch (Alexander, 1974) mit dem extemen Arbeitsmarkt betrachtet. Eine hohe Fluktuation spricht für kurze Verbleibzeiten auf dem intemen Arbeitsmarkt. Hohe Fluktuation widerspricht den qualitativen Eigenschaften intemer Arbeitsmärkte, die durch lange Beschäftigungszeiten (Wolfs, 1990; Schasse, 1990) gekennzeichnet sind.

Interne Arbeitsmärkte sind ökonomischer Ausdruck der Angebots-Nachfrage-Beziehung. Sie tragen dazu bei, durch Arbeitnehmerbindung Angebotsdefizite abzufedern. Ein aus- 
reichendes Arbeitsangebot ermöglicht die Anhebung der 'organizational hiring standards' (Windolf/Hohn, 1984; Cohen/Pfeffer, 1986) an den Eintrittsstellen für neue Organisationsmitglieder. Dies wird in der Absicht vorgenommen, um Kosten der Fluktuation niedrig zu halten. Die Kosten der Fluktuation entstehen dann, wenn die 'organizational hiring standards' einen zu großen Zugang potentieller Arbeitnehmer ermöglichen.

Darüberhinaus ist es denkbar, daß bei einer Verschlechtung der Wirtschaftsentwicklung der interne Arbeitsmarkt ein auf Stabilität basierendes Beschäftigungsrefugium darstellt, das bei veränderten Außenbedingungen nicht sofort zur Reaktion verleitet wird. In diesem Fall verspricht der inteme Arbeitsmarkt sowohl Stabilität als auch Flexibilităt der Beschäftigung (Gabriel, 1985, Atkinson, 1984). Denkbar sind Situationen, die eine vorsichtige interne Steuenung der Beschäftigung erfordem, etwa in der vorübergehenden Entlassung von Personal (Kurzarbeit), in der Kürzung der Ausbildungsaktivităten (Schultz-Wild, 1978; Bills, 1987) oder in Form längerer Verbleibzeiten in Mobilitätsketten.

\subsubsection{Andere Determinanten interner Arbeitsmärkte}

In diesem Abschnitt werden kurz weitere Determinanten intemer Arbeitmärkte angesprochen. Diese haben einen prägenden Einfluß auf die Entstehung bzw. Zustandekommen intemer Arbeitsmärkte.

\subsubsection{Gewerkschaftlicher Einfluß als Determinante}

Ein Grund, warum interne Arbeitmärkte entworfen werden, ist die Chance, gewerkschaftlichen Einfluß zu kanalisieren. Aus den historischen Studien Elbaums (1983) geht hervor, daß inteme Arbeitsmärkte das Resultat gewerkschaftlichen Einflusses sind. Gewerkschaften nehmen in einigen Industriezweigen der Vereinigten Staaten über kollektive Vereinbarungen Einfluß auf die Möglichkeiten intemer Beförderungen (Doeringer/Piore, 1971). Einige aktuellere Untersuchungen (Pfeffer/Cohen, 1984; Bills, 1987; Baron, Davis-Blake und Bielby, 1986) weisen auf die Tendenz hin, interne Arbeitsmärkte einzuführen, um gewerkschaftlichen Einfluß auszuschalten. Diese Untersuchungsergebnisse scheinen eine Bestätigung dafür zu sein, daß Arbeitgeber die Personalpolitik als Teil von Bemühungen benutzen, um den gewerkschaftlichen Einfluß zurückzudrängen (Edwards, 1979). Demgegenüber steht die Perspektive, Gewerkschaften in das Funktionieren interner Arbeitsmärkte einzubeziehen als Ausdruck von Partnerschaft und zur Erzielung eines befriedeten Arbeitsklimas. Ein solche Perspektive geht von einem hohen Grad an Institutionalisienung aus, bei der 'einvernehmliche Lösungen' erreicht werden (Kunst/Soeters, 1991).

Während die eine Perspektive, vorwiegend in Amerika, in der Ausgrenzung gewerkschaftlichen Einflusses besteht, also eine Prioritätenverlagung von Gewerkschaften zum Management (Barbash, 1990) anzutreffen ist, haben Gewerkschaften in Europa vielfach eine institutionalisierte Rolle bei der Gestaltung und Steuerung interner Arbeitsmärkte. Aber auch für die Niederlande sowie allgemein konstatiert Albeda (in: Gijsbers/Sprenger, 1990) Tendenzen in Richtung eines 'unorganisierten Arbeitsmarktes', d. h., daß der Einfluß der Gewerkschaften auf das Personal- und Organisationsgeschehen rückläufig ist. Der gewerkschaftliche Einfluß auf das Zustandekommen interne Arbeitsmärkte könnte damit langfristig ebenfalls abnehmen. 


\subsubsection{Sektor als Determinante}

Ein weitere extrinsische Determinante für die Existenz eines internen Arbeitsmarktes ist der Sektor, in dem sich der interne Arbeitsmarkt befindet. Interne Arbeitsmärkte werden häufiger in ökonomischen Zentren und weniger in der Peripherie angetroffen. Dieser Aspekt läßt sich aus der dualen Arbeitsmarkttheorie ableiten (Althauser/Kalleberg, 1981). Baron u. a. $(1980 ; 1984)$ grenzen diesen Aspekt weiter ein. Diese Determinante interner Arbeitsmärkte ist eher dort anzutreffen, wo es sich um Produktionsuntemehmen und Einrichtungen des 'institutional sector' handelt. Damit sind staatliche Einrichtungen (v. Ours/ Zoethoud, 1990) gemeint bzw. Untemehmen, die durch 'social-overhead-capital' geprägt sind.

\subsubsection{Intrinsische Kennzeichen intemer Arbeitsmärkte}

Interne Arbeitsniärkte variieren mit unterschiedlichen organisatorischen Kennzeichen. Dabei geht es um die Organisationsform interner Arbeitsmärkte und die organisationsstrukturellen Aspekte wie sie beispielsweise in Form organisatorischer Karrieremuster zum Ausdruck kommen (Gunz, 1989). Sofern Organisationen groß und expandierend sind, wird die Wahrscheinlichkeit größer, daß diese Organisationen interne Arbeitsmärkte entwickeln (Rosenbaum, 1979b, 1984; Pfeffer/Cohen, 1984; Wholey, 1985; Baron, Davis-Blake und Bielby, 1986). Skalenerträge, die bei Einrichtung eines intemen Arbeitsmarktes zu erzielen sind, wirken als Anreiz für die Etablierung. Soweit eine Organisation einen Zweigbetrieb repräsentiert innerhalb einer umfangreichen rekursiven Organisationsstruktur, so ist die Wahrscheinlichkeit groß, daß dabei eine inteme Arbeitsmarkt-Praxis vorherrscht (Pfeffer/ Cohen, 1984; Baron et. al., 1986; Gunz, 1988).

Außerdem sind Beschäftigungssysteme wie die des internen Arbeitsmarktes eher dort anzutreffen, wo eine professionelle Personalabteilung ursächlich das Personalgeschehen beeinflußt (Pfeffer/Cohen, 1984; Cohen/Pfeffer, 1986; Baron et al., 1986). Von einer Personalabteilung wird eine Steuerung intemer Arbeitsmärkte, insbesondere das Allokationsmanagement von Mobilitätsketten erwartet sowie die Institutionalisierung von akzeptierten Beschäftigungspraktiken (DiPrete, 1989; 1987b). Darüberhinaus bilden interne Arbeitsmärkte ein ökonomisches Gestaltungsprinzip (Bills, 1987) und ein personalwirtschaftliches Instrument betrieblicher Beschäftigung (Pfeffer, 1985; Kanter, 1984).

Eine weitere Kategorie der Determinanten basiert auf Williamsons (1975) Transaktionskostentheorie. Er argumentiert, daß betriebsspezifische Fähigkeiten, die in einer Art 'leaming on the job' zustande kommen und vielfach nicht $z u$ anderen Arbeitgebem transferierbar sind, eines 'schützenden Rahmens' (protective governance structure) bedürfen (Williamson, 1981). Untemehmen generieren bei Arbeitnehmem betriebsspezifische Qualifikation (Laske, 1987). Sie haben ein Interesse, daß der Arbeitnehmer in der Organisation verbleibt. Fluktuation ist zu teuer, weil neu rekrutiertes Personal diese Qualifikation über einen langen Zeitraum hinweg erst erwerben muß (Wolfs, 1988).

Williamson greift mit der betriebsspezifischen Qualifikation, hier im Kontext intemer Arbeitsmärkte, eine Problematik auf, die in der Bundesrepublik zu Beginn der 70er Jahre durch die Arbeiten des Instituts für Sozialwissenschaftliche Forschung zur Debatte stand. 
Insbesondere die Arbeit von Böhle/Altmann (1972) geht ein auf die sozialen Risiken, die aus ciner einseitigen betriebspezifischen Qualifikation entstehen. Hingewiesen wird auf die Entqualifizierung der Arbeitskräfte, die Beeinträchtigung der Leistungsfähigkeit sowie die Abhängigkeit vom Betrieb. In der Sache wurden durch die Arbeit von Böhle/Altmann die Folgen sowohl für die betroffenen Arbeimehmer als auch für die Gesellschaft untersucht. Ohne die Beschäftigungsform des internen Arbeitsmarktes explizit zu nennen, werden die Beschäftigungswirkungen intemer Arbeitsmärkte antizipiert.

Inteme Arbeitsmarktkonstellationen sind darn wahrscheinlicher, wenn die Qualifikation firmenspezifisch ist (Pfeffer/Cohen, 1984; Baron et al., 1986; Brandes/Buttler, 1989). Auch Organisationskultur (Hofstede, 1980, 1991; Soeters, 1986; Peters/Waterman (1982); (Ouchi/Jaeger, 1978) bzw. spezifischer Organisationsatmosphäre (Spangenberg, 1989) kann zur Bildung bzw. zum Funktionieren intemer Arbeitsmärkte beitragen.

Der interne Arbeitsmarkt bietet Möglichkeiten der Beförderung von Arbeitnehmern. Arbeitgeber geben den Beschäftigten einen Anreiz, im Unternehmen zu verbleiben und über Mobilitätsketten einen höheren Rang zu bekleiden (London/Mone, 1987). Bei ganzheitlicher Betrachtung der verschiedenen Mobilitätsschritte werden Karrieren bzw. Beschäftigungsmuster (Miyazaki, 1977) deutlich. Gunz (1988) spricht in diesem Zusammenhang von den 'organizational career logics' und meint damit die Arbeitserfahrung, die beispielsweise ein Manager im Verlauf seiner organisationsbezogenen 'Karriere' erwirbt. Umfassender, im interorganisationalen Sinne ist das 'work role transition'-Konzept, das mehrere Organisationen (Nicholson, 1984) einbezieht. Ausgefeilte Beförderungssysteme in intemen Arbeitsmärkten tragen dazu bei, diese Beschäftigungsform zu wählen, auch wenn zuweilen ein Bürokratieverdacht entsteht (Halaby, 1978, Becker, 1985; de Grip, 1985; DiPrete, 1989), ohne daß dadurch die Effizienz leidet (Perrow, 1986; Crozier, 1964; Weber, 1972).

Karriereleitem, Mobilitätsketten und andere Formen von untereinander verbundenen Arbeitsplatzfunktionen haben zum Ziel, organisatorisches Lernen (Agyris/Schön, 1978; Fricke, 1975) zu erleichtem. Mit der Mobilitätsstruktur geht vielfach eine Gehaltsstruktur (Konrad/Pfeffer, 1990; DiTomaso, 1988) einher, die die oben erwähnten Funktionen noch verstärken (Wolfs, 1992). Damit sind einige der Determinanten aufgezeigt, die für die Beschäftigungsform des internen Arbeitsmarktes sprechen.

\subsection{Interne Arbeitsmärkte im Lichte der Neuen Institutionellen Ökonomie}

Die Arbeit von Williamson wird in erster Linie mit dem Konzept der Neuen Institutionellen Ökonomie assoziient. Hinter diesem Konzept steht weniger eine neue ökonomische Theorie als vielmehr eine Synthese von neoklassischen und institutionalistischen Theorieelementen (Drugger, 1981/1983). Ausgangspunkt ist die Allokation von Arbeit auf Firmenebene. "... it is the economic institution of the firm which is under scrutiny, and industrial sociology and organisation theory which are the questioned approaches. ... It provides an explanation in broadly neoclassical terms for the size, shape and texture of the firm and its associated labour 'market'." (Francis/ Turk/Willman, 1983, S. 1) 
Zentraler Gegenstand des Konzepts ist die Tauschaktivität auf dem Arbeitsmarkt. Diese kommt zum Ausdruck in den Transaktionskosten, deren begriffliche Grundlage von Coase (1937) und Commons (1931) gelegt wurde. Nach Picot (1982) und Bössmann (1983) sind Transaktionskosten die Kosien, die zur Durchsetzung, Kontrolle und Austausch von Eigentumsrechten anfallen. "... auf den Arbeitsmarkt bezogen sind es jene Kosten, die bei Tauschaktivitäten hinsichtlich der Information, der Aushandlung von Verträgen und der Kontrolle der Leistungserfüllung entstehen. Transaktionskosten dienen als Kriterium der Beurteilung der Effizienz verschiedener Organisationsformen der Arbeitskräfteallokation." (Singer, 1986, S. 301, Fußnote 2)

Williamson (1975) unterscheidet zunächst zwei Formen der Organisienung der Transaktionen von Arbeit. Dies kann zum einen durch den Markt zum anderen durch das Prinzip der intemen Organisation erfolgen. Bei der Marktorganisation ist der Preis ein wesentliches Steuerungselement. Bei Übertragung des Marktprinzips auf den Arbeitsmarkt ist in erster Linie an den extemen Arbeitsmarkt zu denken. Die Marktsteuerung entspricht weitgehend dem Modell der neoklassischen Theorie. Das Prinzip der internen Organisation basiert auf administrativen Regelungen, die zum Ausdruck kommen in der Hierachie, in der Kontrolle und der Entscheidung über die jeweilige Art des Arbeitseinsatzes (Dow, 1987; Baaijens/Have, ten/Josepa, 1989).

Welches der beiden Prinzipien (Markt oder Hierarchie) wirklich zum Zuge kommt, hängt ab von den Transaktionskosten (Groenewegen, 1989). Sind die Transaktionskosten im Fall des Marktversagens (Schreuder, 1983) geringer als im Falle der Marktlösung, so spricht alles für eine Arbeitskraftallokation nach dem Koordinationsprinzip der intemen Organisation oder umgekehrt. Williamson (1975) geht noch weiter und zeigt die Vorteile auf, die die Untemehmung gegenüber dem Markt aufweist. Diese lassen sich kompakt so ausdrücken (Williamson, 1975, S.40):

"1. In circumstances where complex, contingent claims contracts are infeasible and sequential spot markets are hazardous, intemal organization facilitates adaptive, sequential decision making, thereby to economize on bounded rationality.

2. Faced with present or prospective small-numbers exchange relations, internal organization serves to attenuate opportunism.

3. Convergent expectations are promoted, which reduces uncertainty.

4. Conditions of information impactedness are more easily overcome and, when they appear, are less likely to give rise to strategic behavior.

5. A more satisfying trading atmosphere sometimes obtains."

Bei einer differenzierteren Betrachtung fältt auf, daß die Gründe für ein 'Marktversagen' in einer Kombination von subjektiven und objektiven Kriterien des Tausches liegen. Diese lassen sich wie folgt (Williamson/Ouchi, 1983, S. 15) darstellen: 
Abb. 5: Das 'organizational failure framework'
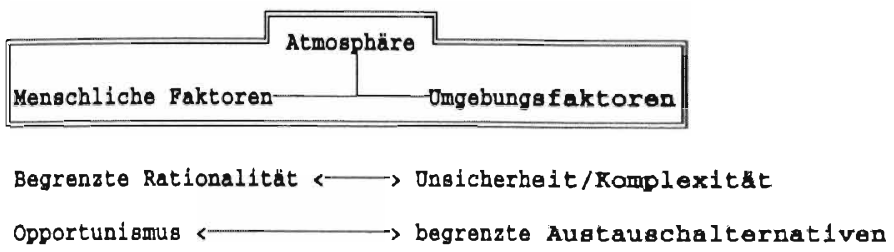

Die 'menschlichen Faktoren' sind Verhaltensannahmen, die bei Williamson/Ouchi zwei Formen annehmen können. Einerseits handelt es sich um die 'begrenzte Rationalität', die darauf basiert, daß eine Situation vollständiger Information nur sehr selten erreichbar ist (Steeck, 1988). Andererseits geht es um opportunistisches Verhalten der 'Tauschpartner', d. h. der Entfaltung egoistischer Neigungen im Sinne von Nutzenmaximalisierung neben einer begrenzten Zahl von Austauschalternativen. Von diesen beiden 'menschlichen Faktoren' leitet Williamson (1981) 'drei Prinzipien des organisatorischen Designs' ab. Sie dienen dazu, die Transaktionskosten der Koordination ökonomischer Aktivitäten zu minimieren. Dabei handelt es sich um das Prinzip des spezifischen Vermögenswertes (asset-specifity-principle), das Externalitätsprinzip (externality principle) und schlieBlich das 'hierarchische Zerlegungsprinzip' (hierarchical decomposition principle). Während die beiden erstgenanten Prinzipen auf das Verhältnis inteme Organisation/Markt gerichtet sind, ist das letzte auf die interne Struktur der Unternehmung zugeschnitten.

Das Zusammenwirken von menschlichen Faktoren und Umgebungsfaktoren kann zu Vertragssituationen führen, in denen über Märkte koordinierte Transaktionen zu höheren Transaktionskosten führen als die im Rahmen einer Organisation koordinierten Transaktionen so wie oben angedeutet.

Die vier Faktoren des 'organizational failure framework' begründen nach Auffassung von Williamson/Ouchi das Versagen des Marktprinzips und den Rückgriff auf das Prinzip der internen Organisation (Willman, 1983). Grob gesehen, läßt sich die interne Organisation mit dem intemen Arbeitsmarkt in Verbindung bringen. Im folgenden soll darauf näher eingegangen werden.

\subsection{1 'Governance structures' der Arbeitsorganisationen}

Bei der Abwicklung von Transaktionen stehen - grob gesehen - zwei Möglichkeiten zur Verfügung. Die eine konzentriert sich auf den 'Markt', die andere auf der Unternehmensebene. Mit den 'governance structures' will Williamson einen weiteren Differenzierungsgrad emeichen. Neben der 'Markt-govemance structure' und der intemen Organisation hat Williamson eine 'Hybrid-governance structure', die zwischen Markt und Hierarchie angesiedelt ist, entwickelt (Williamson, 1991). Williamson weicht damit ab von der in der Literatur anzutreffenden Dichotomie von Markt und Hierarchie. Schreuder (1990) weist auf die Vielzahl von Koordinationsmechanismen in der Realität hin, die nicht unbedingt hierarchischer Natur sein müssen, wohl jedoch dem Prinzip der intemen Organisation entsprechen. 
Im Kontext dieser Arbeit interessiert vor allem die 'govenance structure' auf Unternehmensebene, 'Govemance structures' (Williamson, 1979, S. 239) bilden den institutionellen Rahmen für die Ausführung von 'Transaktionen'. Die Definition der 'governance structure' wird an anderer Stelle (Williamson, 1984) in Bezug auf Arbeitsleistungen spezifiziert.

"The transactions costs approach rests on the proposition that governance structures for labour need to be matched with the attributes of labour transactions in a discriminiating way if transactions costs economizing is to be accomplished."

Als Resultat dieser Differenzierungsbemühung werden von Williamson (1981, S. 549) drei Analyseebenen unterschieden:

Als 'basic unit of analysis' gilt die "...overall structure of the enterprise. This takes the scope of the enterprise as given and asks how the operating parts should be related one to another."

Die 'overall structure' der Untemehmung ist kennzeichnend für die organisatorische Form der Unternehmung. Direkt daran gekoppelt ist die Frage der Untemehmensgröße.

Als zweite analytische Ebene spricht Williamson den 'middle level' an. Der "...middle level focuses on the operating parts and asks which activities should be performed within the firm, which outside it, and why. This can be thought of as developing the criteria for and defining the 'efficient boundaries' of an operating unit."

Diese analytische Ebene richtet den Blick auf das interne Funktionieren der Arbeitsorganisation. Eine in sich weiter unterteilte Struktur ausgehend von der Analyseebene des 'middle level' der 'efficient boundaries' sucht man bei Williamson vergebens. Von der Intention verfolgt Williamson eine strukturelle Abgrenzung innerhalb der Unternehmung im Hinhlick auf funktionale und disfunktionale Aktivitäten. Allein die Zusammenfassung funktionaler Aktivitäten innerhalb der 'effizienten Grenzen' ermöglicht Transaktionskostenvorteile. Die Erwartung, bei Williamson auf der Basis von 'efficient boundaries' (Scott, 1987) ein organisationsintemes Strukturkonzept zu finden, erfültt sich auf diesem Analyseniveau bzw. in diesem Kontext nicht.

Schließlich die dritte Analyseebene "... is concemed with the manner in which human assets are organized. The object here is to match internal governance structure with the attributes of work groups in a discriminating way."

Bei dieser Analyseebene steht nach Williamson die personalwirschaftliche Konkretisierung der Struktur bzw. die Benennung der strukturellen Einheiten im Vordergrund, auf deren Basis die transaktionskostenbezogene Effizienz im Vergleich ermittelt werden kann. Hier kündigt sich ein zentraler Aspekt an, dessen Ausfüllung im Laufe der Arbeit geschehen wird. Richtungsweisend ist hier die organisationsstrukturelle Perspektive und weniger die von Williamson betonte Vertragsperspektive. Der Vertragsperspektive wird insofern Rechnung getragen, als daß von einem impliziten Vertrag ausgegangen wird, der im Zusammenwirken von Arbeitnehmer und Arbeitgeber inhaltlich bestimmt wird. 
Diese drei analytischen Dimensionen erlauben eine erste, wenn auch noch unzureichende Struktureinteilung. Eine weitere differenzierende Ausfüllung ist noch zu leisten. Williamson nimmt diese zum Teil selbst vor. Auch hier richtet sich der Blick ähnlich wie bei den Arbeitsmarkttheorien von der jeweils größeren zu identifizierenden Struktureinheit zur jeweils kleineren Struktureinheit.

Auf der Ebene der Unternehmung bzw. der internen Organisation lassen sich mehrere Arten von

\section{'governance structures'}

unterscheiden. Diese sollen hier kurz vorgestellt werden. Innerhalb der Unternehmung können mehrere 'governance structures' parallel auftreten, weil es sich um 'governance structures' von Transaktionen handelt. Gleichzeitig geht es um die Frage der Konzeptionalisierung der Beschäftigungsbeziehung. "...How are human asset differences best described, what are the employment relation altematives, and what is the appropriate correspondence between them?" (Williamson, 1981, S. 562).

Transaktionen (von Arbeit) umfassen nach Williamson drei Merkmale. Dies sind die Häufigkeit, die Ungewißheit und die Vermögensspezifität. Das Merkmal 'Häufigkeit' ist aufzufassen als ständig wiederkehrende Transaktion.

In der folgenden Abbildung finden sich die identifizierten vier 'govemance structures'.

Abb. 6: Untemehmens-'govemance structures'

\begin{tabular}{|c|c|c|c|c|c|}
\hline & & Hum & co & apital & \\
\hline & & nicht spezifisch & & opezifigch & \\
\hline Arbeits- & leicht & Spotmarkt & 1 & $\begin{array}{l}\text { Wechselseitig ver- } \\
\text { pflichtender Markt }\end{array}$ & 2 \\
\hline uberwachung & $\begin{array}{l}\text { schwie- } \\
\text { rig }\end{array}$ & Binfaches Team & 3 & Beziehungsteam & 4 \\
\hline
\end{tabular}

Quelle: Williamson, 1981, S. 566

Wie entsprechen die vier Formen nun dem intemen Arbeitsmarkt? Obige Abbildung zeigt die Koordinationsformen von Arbeit auf. Williamson unterstreicht, daß die Wahl der 'govemance structure' auch eine Entscheidung über die Effizienz beinhaltet. Schlüssel bei der Identifizienng der 'govemance structure' ist die Firmenspezifizität des Humankapitals und die Messung der Produktivität. Es handelt sich dabei um Kriterien, die eine Grobcharakterisierung ermöglichen und damit einen ersten Zugang ermöglichen. Betriebsspezifische Qualifikationen sind nur schwer auf andere Arbeitgeber zu transferieren. Williamson spricht daher von der geschützten (protective) 'governance: structure', in der die betriebsspezifische Qualifikation eingebunden ist. 
Die in der obigen Abbildung aufgezeigten Koordinationsformen sollen hier kurz erläutert werden. Eine weitergehende Betrachtung unter stärkerer Akzentuierung der Kriterien interner Arbeitsmärkte geschieht weiter unten.

Feld 1 symbolisiert den internen Spotmarkt. Dabei handelt es sich um einen Markt, der durch unspezifisches Humankapital und leichter Messung der Produktivität gekennzeichnet ist. Bei dieser Koordinationsstruktur kann der Arbeitnehmer leicht durch einen anderen ersetzt werden. Anpassungskosten fallen in der Regel nicht an. Eine Arbeitnehmerbindung durch das Management existiert bei dieser Form der 'govemance structure' nicht. Sowohl auf Veranlassung des Managements als auch des Arbeitnehmers kann ein solches Beschäftigungsverhältnis aufgelöst werden.

Feld 2 hat den 'wechselseitig verpflichtenden Markt' zum Gegenstand. Charakterisieren läßt sich dieser durch leichte Ermittlung der Produktivitäl und eine starke Ausprägung furmenspezifischen Humankapitals. Im Gegensatz zum Spotmarkt steht bei dieser Form die langfristige Betriebsbindung im Vordergrund. Arbeitnehmer erwerben ein firmenspezifisches Humankapital. Der Arbeitnehmermobilität (Tigchelaar, 1987) in Bezug auf Untermehmenswechsel sind Grenzen gesetzt. Dies ergibt sich einerseits aus den einseitig erworbenen Fähigkeiten, die nicht ohne weiteres auf ein anderes Unternehmen übertragbar sind, andererseits will der Arbeitgeber durch die Betriebsbindung die firmenspezifischen Kenntnisse durch Abwanderung des Arbeitnehmers nicht gefährdet sehen.

Hier wird deutlich, daß sowohl Arbeitgeber als auch -nehmer ein Interesse an der Kontinuität des Beschäftigungsverhältnisses haben. Die Einlösung der 'wechselseitigen Verpflichtung' besteht von Seiten des Managements in den besonderen Anforderungen an die organisatorische Gestaltung der Arbeitsstrukturen und - soweit entwickelt - einer Organisationskultur. Arbeitsstrukturen beinhalten für Arbeitnehmer eine Chance zur Qualifikationsentwicklung durch intraorganisationale Mobilitätspfade. Dies setzt eine ausgeprägle Hierarchie voraus. Insider genießen gegenüber Outsidem bei der Stellenbesetzung Präferenzen. Der 'wechselseitig verpflichtende Markt' zeigt aufgrund der hier vorgestellten Indikatoren eine Verwandtschaft zum intemen Arbeitsmarkt.

Feld 3 steht für das 'Einfache Team'. Bei dieser Koordinationsstruktur von Arbeit läßt sich die Arbeitsproduktivität nur schwer ermitteln. Das bei dieser Form anzutreffende Humankapital ist meistens nicht firmenspezifisch.

Es handelt sich um Beschäftigte, deren Fähigkeiten also nicht sehr an die Organisation gebunden sind. Die Qualifikationen entsprechen extemen Standards und sind vielfach auch organisationsextem erworben. Zwei Konkretisierungen sind Gegenstand des 'Einfachen Teams'. Zum einen ist es die Zusammenarbeit mehrerer Personen in einem Team im Sinne von Alchian/Demsetz (1972). Für Alchian/Demsetz sind die Beziehungen innerhalb des Untemehmens keine Autoritätsbeziehungen sondem freiwillig eingegangene vertragliche Vereinbarungen. Zum anderen trifft die Charakterisienung der Merkmale auf Personen zu, die dem berufsfachlichen Markt (Sengenberger, 1978) zuzurechnen sind. Bezeichnet werden damit die Angehörigen professioneller Benfe (Williamson, 1984) wie Krankenschwestern, Ärzte, Rechtsanwälte, Techniker und Rechnungsprüfer, um nur einige Beispiele zu nennen. 
Die Effizienzvorteile liegen bei dieser Organisationsform darin, daß mit kleinen Teams gearbeitet wird, in denen wenig Fluktuation herrscht und auf diese Weise 'Shirking' vermieden wird (Balzer, 1987, S. 218 f.). Gruppenspezifische Normen tragen zur Schaffung von "... quasi-moralischen Elementen wie Vertauenswürdigkeit, Loyalität und kooperative Einstellung... .", (Balzer, 1987, S. 219). Die Gruppenarbeitssituation führt bei der Form des 'Einfachen Teams' zu Effizienzvorteilen. Williamson beschränkt sich allein auf diese Form der Konkretisierung des Einfachen Teams (Williamson, 1981). Erst später (1984) werden professionelle Arbeitnehmer nach Auffassung Williamsons dem Spotmarkt zugeschrieben. Diese Zuordnung auf die linke Seite der Abbildung ist auf den ersten Blick gesehen sicherlich richtig. Jedoch aus der Logik der Übersicht ergibt sich als zweiter Blick, daß professionelle Arbeitnehmer unter die Form des 'Einfachen Teams' fallen aufgrund einer theoretisch begründeten schwierigen Leistungsüberwachung sowie aufgrund eines nicht spezifischen Humankapitals.

Die professionellen Arbeitnehmer treten keinesfalls auf unterstem Hierarchieniveau in die Organisation ein. Die Hierarchie bei dieser Organisationsform ist allerdings weniger stark entwickelt als dies bei der in Feld 2 erläuterten Form der Fall ist. Mobilität nimmt daher einen anderen Stellenwert ein. Althauser/Kalleberg (1981) betonen, daß bei den sogenannten 'occupational intemal labour markets' interorganisationale Mobilität intraorganisationale dominiert.

Schließlich bleibt als vierte 'governance structure' die des 'Beziehungsteams' oder in der deutschsprachigen Literatur des 'Qualifizierten Teams' (Balzer, 1987) als Übersetzung für 'relational team'. Diese ist gekennzeichnet durch die Schwierigkeit der Emittlung der Produktivität und einen hohen Grad von Humankapitalinvestitionen. Allein der Zusammenhalt der Gruppenmitglieder dürtte beim 'Qualifizierten Team' größer sein als im Vergleich zum 'Einfachen Team'. Bei dieser Form der 'governance structure'ist auszugehen von einer beträchtlichen 'social conditioning' (sozialer Konditionierung) (Williamson, 1981, S. 565), bei der die Arbeitnehmer in einer besonderen Weise sich dem 'Firmenzweck' widmen.

Als 'Gegenleistung' wird dann auch Arbeitsplatzsicherheit in Aussicht gestellt. Ouchi (1980) spricht von der Clan-Form und ergänzt damit Williamsons Perspektive. Lernprozesse bestimmen die Herausbildung spezifischer Qualifikationen der Arbeitnehmer. Vor diesem Hintergrund ist diese Organisationsform langfristig angelegt. Es liegt im Interesse der beiden Seiten, eine Veränderung der Beschäftigungssitutation möglichst zu vermeiden. Williamson weist auf die Schwierigkeit hin, 'relational teams' zu entwickeln. Sie werden in japanischen Unternehmen vermutet.

Die vier 'govemance structures' können in unterschiedlicher Gewichtung parallel in einer Organisation bestehen. Dieser Tatsache wird insofern Rechnung getragen, daß zwei 'govemance structures' im Rahmen der empirischen Untersuchung berücksichtigt werden, da diese trotz jeweils unterschiedlicher Ausprägung von Beschäftigungsmerkmalen auch gemeinsame aufweisen. Eine weitere Konsequenz aus der parallelen Existenz von 'governance structures' ist die spezifische Beobachtung der Merkmalsausprägungen im Rahmen der empirischen Erhebung. Außerdem wird im Rahmen der Untersuchung geprüft, inwieweit mögliche Anhaltspunkte für die Existenz der hier nicht gewählten 'governance 
structures' vorhanden sind. Dies geschieht in Kapitel 6 und an Hand von Merkmalsausprägungen in Kapitel 7 und 8. In diesen Fällen geht es um die Erschließung des Umfangs. Ansonsten wird für den weiteren Verlauf dieser Studie davon ausgegangen, daß in der Realität eine bestimmte 'governance structure' dominiert.

Eine weitere Konsequenz besteht in der Einbeziehung einer weiteren Organisationstheorie, von der eine analytische Spezifikation von Beschäftigungsmerkmalen in Bezug auf Organisationstypen erwartet wird. Ziel ist es, die Beschäftigungsmerkmale des Organisationtyps in Verbindung zu bringen mit den 'governance structures'. Wir werden an anderer Stelle darauf zurück kommen.

\subsubsection{Die Organisationsstruktur bei der Transaktion von Arbeit}

Frkenntnisleitend ist bei Williamson die Konkretisierung der Organisationsstruktur (Transaktionsstruktur). Dies läßt sich nicht nur aus dem Begriff 'govemance structure' ableiten sondem auch aus den damit verbundenen Ideen. Die typischen Organisationsmerkmale der Beschäftigungsbeziehung 'interner Arbeitsmarkt' bestehen aus den Regeln, die den Grad der Offenheit bzw. Geschlossenheit der 'ports of entry' ausmachen und neben anderen die 'job ladders'.

Auf der Ebene der Beschäftigungssituation (employment relation) sind in Bezug auf den intemen Arbeitsmarkt dann Effizienzvorteile zu realisieren, wenn die folgenden drei strukturellen Eigenschaften im Rahmen eines 'Systems' interner Arbeitsmarkt umgesetzt werden (Williamson/Wachter/Harris, 1975).

- Eine Eigenschaft besteht in der Bindung der Gehaltsstufen an Arbeitsplätzen und nicht an die Person des Stelleninhabers. Dies senkt die Kosten des Aushandelns von Verträgen. Auch die Form des Kollektivvereinbarung trägt dazu bei, eine komplexe Austauschbeziehung in eine 'institutionalisierte' Form. (Putterman, 1987) zu bringen.

- Eine weitere Eigenschaft richtet sich auf die Erzielung eines kooperativen Verhaltens der Beschäftigten, die konkretisiert wird durch eine Anreiz-Beitrags-Bezahlung auf transaktionsspezifischer Grundlage (Ham/Paauwe/Williams, 1988).

- Außerdem gehört dazu eine interne Befördenungsleiter, die Anreiz bietet, daß Personen befördert werden. Beförderungen sollen mit Einschränkung erfolgen. Einschränkung ist auch das Prinzip des Zugangs zur jeweiligen Organisation über die 'ports of entry'. Organisationsintern will man sich vor Personen schützen, die mit geringerer Produktivität und upportunistischem Verhalten die Untemehmung versuchen 'auszubeuten' (exploitation).

Hiermit erschöpft sich das Niveau der Konkretisierung der Organisationsstruktur. Dies geben Williamson, Wachter und Harris (1975, S. 275) in Form eines 'Einspruchs' zu. Die Diskussion "... does not pretend to be exhaustive in describing the structured aspects of intemal labor markets." 
Festzuhalten bleibt, daß die weitere Konkretisierung der 'governance structure' in Form der Arbeitsmarktstrukturdeterminanten (ports of entry, promotion ladders und ein institutionalisiertes Rahmenwerk in Form des Kollektivvertrages) erfolgt, die letztlich auf Kerr bzw. Doeringer/Piore zurückgehen. Die aus der Transaktionskostentheorie ableitbaren Organisationsstrukturen lassen sich nur beschränkt konkretisieren. Daraus wird für den weiteren Verlauf der Arbeit die Konsequenz gezogen, bei der Erschließung der organisationspezifischen Beschäftigungskennzeichen mehr auf die Kontingenztheorie zu setzen.

Aufgegriffen wird die im 'Organisationsrahmenkonzept' zu Beginn dieses Kapitels entwickelte Idee unterschiedlicher Allokationsgrenzen innerhalb der Organisation. Dieser Ansatz soll zu einer empirischen Überprüfung führen. Er konkretisiert sich in der Frage, inwieweit beeinflussen innerorganisatorische Grenzen die interne Wiederbesetzung offener Stellen auf internen Arbeitsmärkten?

Zu fragen ist, inwieweit die Kontingenztheorie mehr Ansatzpunkte für eine Erschließung organisationsstrukturtypischer Beschäftigungsmerkmale bietet bzw. Gemeinsamkeiten mit dem internen Arbeitsmarkt aufweist?

\subsection{Interne Arbeitsmärkte im Lichte der Kontingenztheorie}

Als weitere Organisationstheorie, die in dieser Arbeit Anwendung findet, soll im folgenden auf die Kontingenztheorie eingegangen werden. Sie soll die Transaktionskostentheorie ergänzen.

\subsubsection{Zum Inhalt und Entwicklung der Kontingenztheorie}

Ein Synonym für die Kontingenztheorie ist die Bezeichnung 'Situativer Ansatz' (Kubicek/ Welter, 1985). Im Mittelpunkt stehen Organisationsstrukturen, die von anderen Größen als die der 'governance structures' abhängig und zu erklären sind. Die Kontingenztheorie problematisiert Organisationsstrukturen aus einer bestimmten Situation heraus. Diese Organisationstheorie ist vielfach ahistorisch. Aufgrund dessen bleiben die Analysen an der Oberfläche, ein besonderer Tiefgang im Sinne der Erfassung und Problematisierung von Wirkungszusammenhängen wird meistens nicht erzielt. Allerdings hat diese Theorie eine Entwicklung durchlaufen, bei der verschiedene Theorieversionen entstanden sind, die einen Teil der geäußerten Kritik verblassen lassen. Kubicek/Welter betonen den Gebrauchswert der Theorie für empirische Untersuchungen mit Bezug auf Organisationsstrukturen. Dies gilt auch für die Erfassung des Verhaltens von Organisationsmitgliedem sowie der Erfassung von Organisationseffizienz. Organisatorische Gestaltung soll über die Beeinflussung der 'Struktur' zu bestimmten beabsichtigten Wirkungen führen. Ziel ist die Entsprechung von Modell und Wirklichkeit.

Die Kontingenztheorie ist in der Forschungstradition der vergleichenden Organisationsforschung (Lammers, 1987) zuzuordnen. Sie hat ihre Wurzeln in der 'europäischen' Organisationssoziologie sowie im Human-Relation-Ansatz. In den 50er Jahren beschäftigten sich Organisationssoziologen mit dem Bürokratiemodell Max Webers. Der Vergleich von Webers theoretischem Modell mit der Realität gab der Theoriekonstituierung deutliche Impulse. Ein entscheidender Anstoß erfuhr die Theorie durch die Arbeiten von Bums und 
Stalker (1961), die das BürokratiemodelJ unter Hinzuziehung der Perspektiven des HumanRelations-Ansatzes relativierten. Damit wurde eine Entwicklung eingeleitet, die weitere Impulse erfuhr aus drei Theorierichtungen. Dabei handelt es sich um die Industriesoziologie (Udy, 1962), die Organisationssoziologie Hall (1963); Hage, Aiken (1969) sowie die 'Aston-Gruppe' um Pugh und Hickson (1976) und die anglo-amerikanische Managementlehre mit Woodward (1965), Lawrence/Lorsch (1967/1969), um nur einige zu nennen. Die Kontingenztheorie hat auch Eingang in die Betriebswirtschaftslehre (Grochla, 1982) gefunden. Einen arbeitsmarkttheoretischen Theoriezweig sucht man vergebens.

Kubicek/Welter verweisen auf die Tatsache, daß in der Kontingenztheorie keine einheitliche Definition von Orgarisationsstrukturen besteht, vielmehr wird mit der Zielrichtung der Untersuchung von einem Vorverständnis ausgegangen, das ein breites Spektrum umfassen kann. Dies geht von der Erfassung von Regelungen bzw. Regeln bis hin zu der Perspektive der Erfassung von Organisationstrukturen als Systeme von VerhaltensregelmäBigkeiten. Diese lassen sich quantitativ und qualitativ erfassen. Um dennoch eine Eingrenzung vorzunehmen, greifen wir auf das Konzept der Organisationskonfigurationen von Mintzberg zurück.

\subsubsection{Das Organisationskonfigurationskonzept von Mintzberg}

Das Werk von Mintzberg $(1979,1983,1989)$ läßt sich dem Kontingenzdenken zurechnen. Mintzberg hat die theoretischen und empirischen Zweige der Literatur zusammengeführt hinsichtlich Koordination, Design und Strukturierung von Organisationen. Das Ergebnis umfaßt eine Typologie von sechs Organisationskonfigurationen. Im einzelnen handelt es sich um die 'Einfache Struktur' (simple structure), die Maschinenbürokratie, die professionelle Bürokratic sowie die divisionale Form neben der Adhocratie und der 'missionary form'.

Die Organisationskonfigurationen repräsentieren eine theoretische Ausgangsbasis durch Operationalisierung der Teile der Organisation sowie der Wirkungszusammenhänge für eine empirische Untersuchung.

Die Anwendung des von Mintzberg zur Verfügung gestellten Instrumentariums für empirische Zwecke nimmt zu. Dazu rechnen die Untersuchung von Soeters/Schwan (1990), Langley (1989), Paauwe, (1989), Schreuder u. a. (1988), Schmid (1988), und Maynt7. (1985), um nur einige zu nennen. Erste Ansätze, Mintzbergs theoretische Ausführungen auf den Arbeitsmarkt zu konzentrieren, sind bei ten Have/Vissers (1987) und van Ham/Paauwe/Williams (1987) zu finden. Die Anwendung des Mintzberg-Instrumentariums im Kontext der Betrachtung des internen Arbeitsmarktes stellt ein innovatives Moment dar, da bislang organisationsanalytische Instrumente keine Anwendung fanden in Bezug zum intemen Arbeitsmarkt. 


\subsection{Integration der Arbeitsorganisationsperspektive}

\subsubsection{Die Organisationskonfigurationen}

In Kapitel 1 wurden die Organisationskonfigurationen definiert. An dieser Stelle soll eine kurze Beschreibung der Organisationskonfigurationen erfolgen, die im Kontext der Arbeit relevant sind. Als solche werden die Maschinenbürokratie und die professionelle Bürokratie als Organisationskonfiguration näher betrachtet, da diese Organisationskonfigurationen neben anderen (Soeters/Schwan, 1990), so die Annahme, eine deutlichere Affinität zur Organisationsform des intemen Arbeitsmarktes aufweisen als andere Organisationskonfigurationen (Tirole, 1986).

Seit einiger Zeit belegt Mintzberg (1989) die Organisationskonfiguration der Maschinenbürokratie mit dem Begriff 'Maschinenorganisation'. Inwieweit dadurch das Element 'Bürokratie' verdrängt wird, bleibt einer gesonderten Überprüfung vorbehalten. Idealtypische Beispiele sind Produktionsuntemehmen und Sozialverwaltungen. Diese Organisationsform hat einen hohen Grad an Spezialisierung sowohl in horizontaler als auch in vertikaler Richtung. Die Organisationsstruktur ist hierarchisch und pyramidenförmig aufgebaut. Kennzeichnend sind die formalisierten Regelungen, Programme und Verfahrensweisen. Im Grunde besteht bei dieser Organisationsform in der theoretischen Ausgangsposition Mintzbergs kaum Bedarf an professionellen Ausbildungsprogrammen bzw. Unterweisung. Allerdings ist training-on-the-job deutlich vorherrschend. Die Aktivitäten des Managements sind reduziert auf die Feinabstimmung, die Koordination von Funktionen und die Konfliktlösung.

Typisch für diese Organisationsform ist auch der Entscheidungsfluß von oben nach unten etwa in der Weise wie sie dem Autoritätsfluß entspricht. Die Organisation befindet sich in der Regel von einer stabilen Umgebung und hat einen hohen 'Reifegrad'. Unternehmungen dieser Art entsprechen in der Regel Großunternehmen.

Die Organisationskonfiguration der professionellen Bürokratie, in der neueren Diktion 'Professionelle Organisation' (Mintzberg, 1989) genannt (Beneviste, 1987), ist beispielsweise typisch für Universitäten, Krankenhäuser und Architektenbüros. Wie der Name unterstellt, sind bei dieser Organisationform ebenfalls bürokratische Züge anzutreffen. Nach außen kennt diese Organisationsform eine stabile Umgebung. Planung und Kontrolle sind auf ein relativ geringes Niveau beschränkt.

\subsubsection{Integration der Arbeitsorganisationsperspektive von Williamson und Mintzberg}

Die kurze Charakterisierung der Organisationskonfigurationen von Mintzberg fordert den Vergleich mit den 'govemance structures' von Williamson heraus. Der Anspruch der weitgehend fehlenden Spezifizierung der Organisationsstruktur bei den 'governance structures' soll damit eingelöst werden. Bevor dieser Schritt untemommen wird, sollen zunächst die Äquivalente in den Konzepten des jeweiligen Autors herausgefunden werden. Dazu bedienen wir uns der von Williamson entwickelten Rahmenbedingungen der 'governance structure'. 
Vergleicht man nun die Organisationskonfigurationen Mintzbergs im Rahmen der Maßstäbe Williamsons, dann ergibt sich eine weitestgehende Ähnlichkeit oder in Webers (1976) Terminologie: Wahlverwandtschaft. Geht man dann einen Schrilt weiter, so lassen sich beide Ansätze in das Rahmenkonzept Williamsons nahezu integrieren. Die folgende Abbildung zeigt die Integration des Ansatzes von Williamson und Mintzberg auf:

Abh. 7: Arbeitsorganisationsperspektive von Williamson und Mintzberg

\begin{tabular}{|c|c|c|c|}
\hline & \multicolumn{2}{|c|}{ Bumankapital } \\
\hline & & nicht opezifisch & spezifigch \\
\hline Arbeita- & lelcht & $\begin{array}{l}\text { Spotmarkt } \\
\text { Binfache Struktur }\end{array}$ & 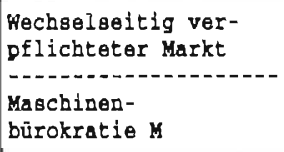 \\
\hline überwachung & $\begin{array}{l}\text { schwie- } \\
\text { rig }\end{array}$ & \begin{tabular}{|l} 
Einfaches Team \\
Profesoionelle \\
Bürokratio M
\end{tabular} & $\begin{array}{l}\text { Beziehungsteam } \\
\text { Missionarische } \\
\text { Form/Adhocratie M }\end{array}$ \\
\hline
\end{tabular}

Quelle: Williamson, 1981; Mintzberg, 1979; Soeters/Schwan, 1990

Den oberen Teil jeder der vier Einheiten beschreibt Williamsons Konzept. Der untere Teil charakterisiert die Organisationskonfiguration von Mintzberg (markiert mit einem M). Die beiden Einteilungskriterien vereinfachen grob. In der Realität sind oft Situationen anzutreffen, aus deren Komplexität eine andere Einschätzung resultieren kann.

Bezogen auf die Beschäftigungsstrukturen bzw. auf Kriterien des internen Arbeitsmarktes lassen sich in Bezug auf die obige Abbildung folgende SchluBfolgerungen ableiten.

Beim Spotmarkt herrscht eine lose Arbeitsteilung vor, ohne daß spezifische Regein und Verfahren eine 'Institutionalisierung' erkennen lassen. Mintzbergs 'Einfache Struktur' entspricht am meisten dem Spotmarkt. Der Einfluß von Kriterien des internen Arbeitsmarktes ist nicht sehr wahrscheinlich. Das Personal kann schnell ersetzt werden durch neue Rekrutierungen auf dem externen Arbeitsmarkt. Ausgenommen davon sind diejenigen, die Aufsicht ausüben. Kriterien des internen Arbeitsmarktes treffen schon deshalb bei dieser Organisationsform nicht $\mathrm{zu}$, da dicse Organisationen recht klein sind (Elbaum, 1983; Pfeffer/Cohen, 1984).

So wie an anderer Stelle festgestellt, ist die Überwachung der Produktivität in dem 'Einfachen Team' theoretisch gesehen schwierig. Die Qualifikation der Personen, die dieser Organisationskonfiguration zugerechnet werden, hat einen hohen Grad, der allerdings nicht organisationsgebunden ist. Bei dieser Organisationsform sind die Kriterien, die auf inteme Arbeitsmärkte schließen lassen, nicht sehr stark ausgeprägt. Nach der Definition von Lutz (1987) sind 'ports of entry' für neue Mitarbeiter zu erwarten. Diese 
sind bei dieser Organisationskonfiguration kaum zu finden. Dies läßt sich aus dem Fehlen zentraler 'ports of entry' im unteren Teil der Hierarchie ableiten.

Ein weiteres Kriterium des internen Arbeitsmarktes ist eine arbeitsplatzbezogene Qualifikations-, Verantwortungs- und Verdienstabstufung sowie hierarchiebezogene Mobilitätsmuster. Die Hierarchie reduziert sich auf Aufsichtsniveaus. Die genannten Kriterien sind schwach ausgeprägt. Die wechselseitige Bindung zwischen Betrieb und Beschäftigten kann für diese Organisationsform unterstellt werden. Das Allokationsmuster hat einen hohen Grad an Verbindlichkeit. Mintzbergs 'professionelle Bürokratie' entspricht dieser 'governance structure'. Althauser/Kalleberg (1981) sprechen dann auch in der analytischen Differenzierung firmenintemer Arbeitsmärkte vom 'occupational internal labor market', bei dem interorganisationale Mobilität deutlich überwiegt (Loveridge, 1983).

Der 'wechselseitig verpflichtende Markt' zeigt Übereinstimmungen mit der Maschinenbürokratie. Hier dominiert eine strukturelle Differenzienung der Arbeitsorganisation mit einem hohen Grad an Institutionalisierung. Die Qualifikation der Beschäftigten ist relativ stark fumenspezifisch und deshalb schwierig übertragbar auf andere Unternehmen. Hier trifft man auf gut entwickelte Jobleitern, über die die Beförderung des Arbeitnehmers erfolgt. Diese beginnen bei den 'ports of entry' und finden ihre Fortsetzung auf den verschiedensten Hierarchieniveaus. Außerdem herrschen Senioritätsprinzip und Aus- bzw. Weiterbildungsaktivitäten, gekoppelt an der Jobstruktur, vor (Pfeffer, 1982). Die Kriterien des intemen Arbeitsmarktes sind hier idealtypisch entwickelt. Diese Organisationsform entspricht idealtypisch dem 'firm internal labor market' (Althauser/Kalleberg, 1981, S. 130), der "...denotes a subcategory of internal labor market - one that is internal to a firm."

Die Organisationsform des Beziehungsteams (Clan) (Williamson/ Ouchi, 1983) bzw. der 'missionary form' bzw. 'adhocracy' bei Mintzberg zeigt eine Dominanz firmenspezifischer Qualifikationen aufgrund von Lemprozessen. Durch die interne Dynamik und die unsichere Umgebung sind die Arbeitsleistungen nur in beschränktem Grad meßbar. Beide Seiten, Arbeitgeber und Arbeitnehmer, haben ein Interesse an ein langfristiges Beschäftigungsverhältnis, um Verluste zu vermeiden, die als Folge interorganisationaler Mobilität auftreten können.

\subsubsection{Arbeitsorganisationstypen im Vergleich}

Die hier vorgestellten Arbeitsorganisationstypen werden vor dem Hintergrund einer erweiterten Literaturperspektive teils bestätigt teils ergänzt oder erweitert. In einer synoptischen Übersicht soll dies aufgezeigt werden. $\mathrm{Zu}$ beachten ist, daß jeder Ansatz seine eigenen 'Nuancen' hat. Dennoch sind die Konturen so gezeichnet, daB ein Vergleich möglich ist. Äquivalente als Ausdruck unterschiedlicher Arbeitsorganisationstypen abgeleitet aus Organisations- und Arbeitsmarkttheorie werden aufzeigt. 
Abb. 8: Synopse vergleichbarer Arbeitsorganisationstypen nach Autoren

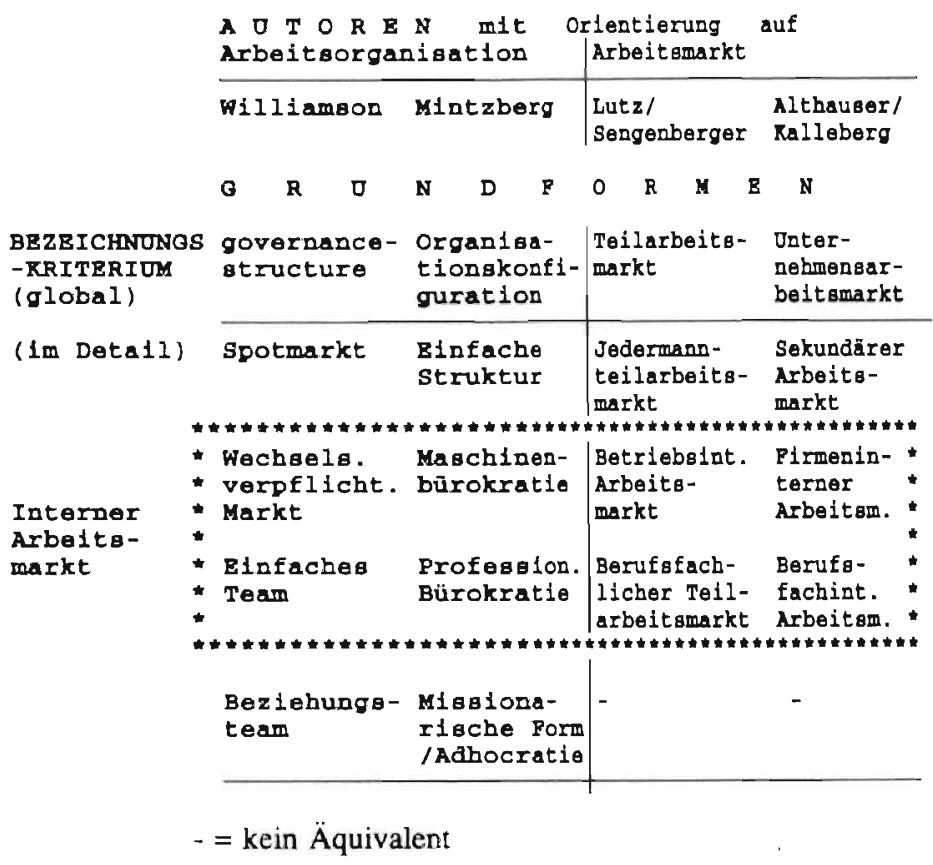

Auf die Erläuterung der 'Typologie' von Williamson und Mintzberg soll hier verzichtet werden, da diese an anderer Stelle ausführlich behandelt wurden. Williamson und Mintzberg repräsentieren auch terminologisch einen Organisationsansatz, während bei den beiden anderen Autorenpaaren mehr eine Arbeitsmarktorientienung in der Bezeichnung zum Ausdruck kommt.

Lutz und Sengenberger (1974); Sengenberger, (1975) haben ein Konzept von Teilarbeitsmärkten entwickelt. Innerhalb des Arbeitsmarktes werden drei Teilarbeitsmärkte identifiziert. Kriterium der Differenzierung ist die Qualifikation.

Der Jedermann-Teilarbeitsmarkt umfaßt Personen, die über eine allgemeine Qualifikation verfügen. Eine spezifische Qualifikation in Form der benfsfachlichen Qualifikation weisen die Personen auf, die dem berufsfachlichen Teilarbeitsmarkt zugerechnet werden. Schließlich verbleibt der betriebsinterne Teilarbeitsmarkt als Ausdruck der Vermittlung betriebsspezifischer Qualifikation.

Althauser/Kalleberg (1981, S. 129 ff.) vertreten einen differenzierteren Ansatz als der, der in der obigen Übersicht zum Ausdruck kommt. Aus Gründen der Vergleichbarkeit soll an der Übersicht festgehalten werden. Dennoch soll der umfassendere Ansatz von Althauser/ Kalleberg hier kurz vorgestellt werden. Grundlage der Differenzierung der Arbeitsmarktformen - wie in Kapitel 1 erwähnt - sind die drei Wesensmerkmale intemer Arbeitsmärkte. 
Diese bestehen aus der Jobleiter, dem Eintritt im unteren Hierarchiebereich sowie der Mobilitätsentwicklung über die Jobleitern.

Beim sogenannten furmenintemen (firm intemal labor market) Árbeitsmarkt treffen alle Wesensmerkmale zu. Beim berufsfachlichen internen Arbeitsmarkt (occupational internal labor market) gibt es Überschneidungen zum fïmeninternen. Die Berufsinhalte sind prinzipiell nicht auf einen Arbeitgeber fixiert. Darüberhinaus werden zwei Formen unterschieden, deren Differenzierungskriterium darin besteht, daß die oben erwähnten drei Wesensmerkmale nur zum Teil zutreffen. Mit Bezug auf die obigen Arbeitsmarktformen lassen sich 'Firmenarbeitsmärkte' (firm labor markets) von 'beruflichen Arbeitsmärkten' (occupational labor markets) unterscheiden. Als Restkategorie, soweit die Arbeitsplätze sich nicht den genannten vier Arbeitsmarktformen zurechnen lassen, gilt der sekundäre Arbeitsmarkt (secondary labor market).

Die Koexistenz verschiedener Arbeitsmarkttypen innerhalb eines Unternehmens unterstreicht auch Ostermann (1982). Er unterscheidet 'industrial, craft und secondary subsystems', die im wesentlichen zu vergleichen sind mit den oben genannten Formen. 1987 fügt Osterman seinen 'employment subsystems' noch ein weiteres viertes 'subsystem' zu, das er 'salaried subsystem' nennt. Das 'salaried model' ist zu vergleichen mit dem der professionellen Bürokratie. Seine Hauptkennzeichen bestehen in Beschäftigungssicherheit, Jobleitern sowie Beförderungsmöglichkeiten. Das 'employment subsystem' schließt eine Lücke, die in Ostermans 1982 Perspektive zweifellos vorhanden war.

Zusammenfassend läßt sich festhalten: die Organisationskonfigurationen Mintzbergs können mit den 'govemance structures' Williamsons in Verbindung gebracht werden. Der Vergleich von Arbeitsorganisationstypen hat aufgezeigt, daß neben den organisationstheoretisch abgeleiteten Typen Äquivalente aus arbeitsmarkttheoretischer Sicht bestehen. Die ebenfalls aufgezeigten Überschneidungen rechtfertigen die Einbeziehung von unterschiedlichen Organisationskonfigurationen. Die obige Abbildung deutet in recht globaler Weise darauf hin, daß Maschinenbürokratien mehr den Beschäftigungskennzeichen intemer Arbeitsmärkte entsprechen als professionelle Bürokratien. Der Nachweis, inwieweit dies so ist, soll in Form der empirischen Untersuchung erbracht werden.

Theoretisch ist die eindeutige Abgrenzung einer dominanten Arbeitsorganisationform nicht vorzunehmen. In der Empirie dürten Überschneidungen an der Tagesordnung sein. Althauser/Kalleberg betonen aus arbeitsmarkttheoretischer Sicht die Arbeitsmarktformen des furmenintemen Arbeitsmarktes und des berufsinternen Arbeitsmarktes. Demgegenüber stehen aus organisationstheoretischer Sicht die Äquivalente von Williamson und Mintzberg. Die Konzeptionen von Althauser/Kalleberg und Williamson konkretisieren jedoch nur unzureichend die 'Infrastruktur' intemer Arbeitsmärkte. Dies setzt eine Erschließung von Beschäftigungskennzeichen voraus. Auch Mintzberg trägt den Beschäftigungskennzeichen nicht implizit Rechnung. Mintzberg bietet jedoch das know how für die ErschlieBung von Organisationskonfigurationen. Damit ist ein Ansatz gegeben, um beschäftigungsrelevante Kennzeichen abzuleiten. Aufgrunddessen konzentriert sich das Erkenntnisinteresse im folgenden auf die beiden Organisationskonfigurationen von Mintzberg. 
Das Erkenntnisinteresse richtet sich auf die unterschiedliche Ausprägung von Beschäftigungskennzeichen intemer Arbeitsmärkte im Rahmen der Organisationskonfigurationen. Die empirische Untersuchung kann darüber definitiv Aufschluß bieten. Dazu sind zwei Schritte erforderlich. Zum einen gilt es zunächst, aus der Literatur Beschäftigungskennzeichen in Verband $\mathrm{zu}$ bringen mit der jeweiligen Organisationskonfiguration. Zum anderen soll zur weiteren Konkretisierung auf das Instrumentarium Mintzbergs zurückgegriffen werden, um über Organisationskriterien Beschäftigungskennzeichen intemer Arbeitsmärkte zu erschließen.

\subsubsection{Erschließung von Beschäftigungskennzeichen}

Im folgenden werden die allgemeine Kennzeichen der jeweiligen Organisationskonfiguration auf der Basis der Literatur synoptisch gegenübergestellt. Der damit verbundene Zweck beruht auf einer ersten Sichtung allgemeiner und beschäftigungsrelevanter Kennzeichen. Außerdem dient diese Übersicht als Hintergnundinformation für die qualitative Darstellung der empirischen Ergebnisse in Kapitel 6.

Mintzberg (1989) schreibt den Organisationskonfigurationen bestimmte Strategien zu. Bei der Maschinenbürokratie handelt es sich um die Status quo-Strategie, die auf Fortschreibung des gegenwärtigen Status basiert, während in der professionellen Bürokratie die autonome Strategie anzutreffen ist, die vom Wissen und know how der Organisationsmitglieder beeinflußt wird. Bei Maschinenbürokratien findet sich in der Regel eine lebenslange Beschäftigung, während die professionelle Bürokratie eher von Beschäftigungswechseln gekennzeichnet ist. In beiden Organisationskonfigurationen finden sich erschlossene Jobstrukturen, die meistens in Form der Jobleitern zum Ausdruck kommen. In der Länge der Jobleitern unterscheiden sich beide Organisationskonfiguration deutlich. Die Länge richtet sich nach der Zahl der Funktionen. Die straffe Kontrollstruktur bei Maschinenbürokratien zielt auf eine Effizienzkostenorientienung. Bei der professionellen Bürokratie dominiert vielfach die Wissens- bzw. Technologieorientierung. Hinsichlich der Automation sind in der jeweiligen Organisationskonfiguration unterschiedliche Konsequenzen verbunden. Bei Maschinenbürokratien wird in der Regel Personal ersetzt, dagegen steht bei professionellen Bürokratien der Arbeitsmitteleinsatz im Vordergrund.

Arbeitnehmermobilität ist gegensätzlich ausgeprägt bei den Konfigurationen. Während bei der Maschinenbürokratie die intraorganisationale Form dominiert, gilt für die professionelle Bürokratie die interorganisationale. Damit im Zusammenhang steht logisch konsistent das jeweilige Rekrutierungsmuster mit mehr intemen Rekrutierungen bei den Maschinenbürokratien und relativ mehr externen Rekrutierungen bei der professionellen Bürokratie. Die Qualifikationsinhalte sind ebenfalls gegensätzlich. Maschinenbürokratien lassen sich kennzeichnen als hierarchisch kontrollierte Organisationen im Gegensatz zur professionellen Bürokratie, in der Vertrauen die gegenseitige Verpflichtung zwischen Arbeitnehmer und -geber ausmacht. Aus dieser Unterschiedlichkeit resultieren dann auch verschiedene Führungsstile, die geprägt werden durch die organisationsstrukturelle Anordnung. 
Tab. 1: Allgemeine Kennzeichen der Organisationskonfigurationen

MASCHINENBÜRORRATIE

Status quo-strategie (Mintzberg, 1989)

'lebenslange' Beschaftigung (Sengenberger, 1987)

erschlosene Jobstruktur, lange Jobleitern

(Baron et al., 1986)

Bffizienzkostenorientierung (Williamson, 1981)

Automation=Brsetzung von Perbonal

intraorganisationale Arbeitnehmermobilität (Pfeffer/Cohen, 1984, Althauser/Ralleberg, 1981)

mehr interne Rekrutlerung (Sengenberger, 1987)

organisationsopezifische Qualfikationsinhalte (Doeringer/Piore, 1971)

bierarchieche Rontrolle (Bdwards, 1979)

autokratischer Führungsstil (Wunderer, 1985)

stark vertikal zentralisiert, beschränkt horizontal dezentralisiert (Mintzberg, 1979)

pflichtgemaßes Handeln (Derfunctory performance)

(Williamson, 1981)
PROFESSIONELLE BUROKRATIE

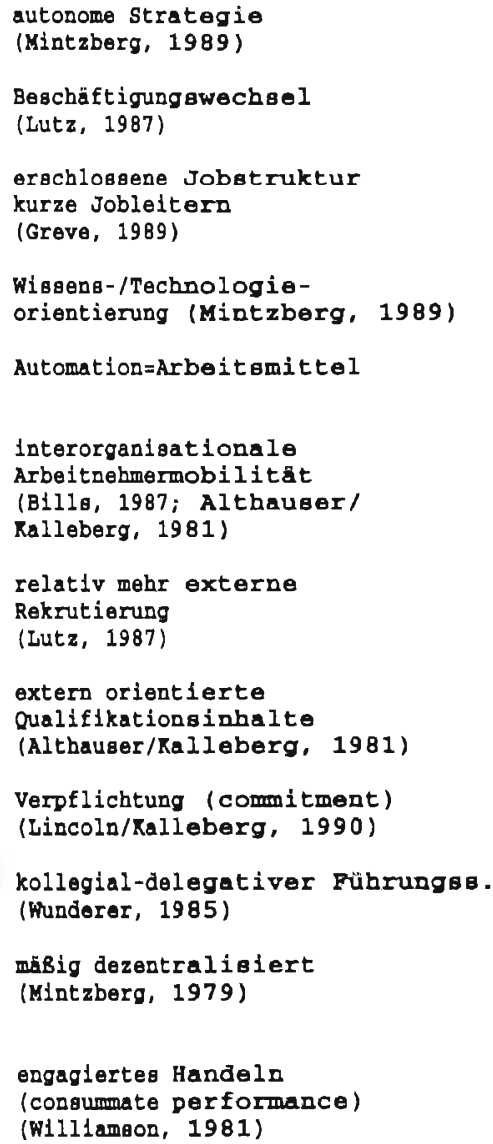

Beim Vergleich der beiden Organisationskonfigurationen wird das eigenständige Profil einer jeden organisatorischen Form der Beschäftigung deutlich.

Charakteristiken des internen Arbeitsmarktes lassen sich auf diese Organisationskonfigurationen zunächst vorläufig - wenn auch in unterschiedlicher Ausprägung - beziehen.

Schlußfolgerungen werden erst nach einer empirischen Unterbauung gezogen.

\subsection{Die Allokation auf internen Arbeitsmärkten}

Bislang stand die Strukturperspektive im Vordergrund der Betrachtung der vorhergehenden Abschnitte. Mit der Allokation, also der Zuordnung von Personen zu Arbeitsplätzen auf 
internen Arbeitsmärkten soll das Geschehen, d. h. die Dynamik am internen Arbeitsmarkt aufgegriffen werden. Zwei Allokationsaspekte stehen im Vordergrund. Zum einen geht es um die Möglichkeit, als potentieller Arbeitnehmer Zugang zu einem intemen Arbeitsmarkt zu finden. Zum anderen wird die interne Reallokation angesprochen. Diese trifft auf Personen zu, die sich innerhalb der Organisation befinden und durch Befördenung, Versetzung oder aus anderen Gründen einen anderen Arbeitsplatz einnehmen.

Dieser Abschnitt gliedert sich wie folgt: zunächst wird der Organisationszutritt als Gegenstand strategischer Beherrschung betrachtet. Diese etwas globale Perspektive wird spezifischer im Hinblick auf Rekrutierungsstrategien (Windolf/Wood, 1988). Damit wird die Beziehung interner - externer Arbeitsmarkt angesprochen. In Bezug auf die Reallokation, d. h. die organisationsinterne Allokation von Arbeitskräften werden die zuvor entwickelten Arbeitsmarktstrukturdimensionen erneut aufgegriffen und zu einem Konzept von Allokationsgrenzen verarbeitet, das bei der empirischen Untersuchung der offenen Stellen und der intraorganisationalen Mobilität von Bedeutung sein wird.

\subsubsection{Organisationseintritt als Gegenstand strategischer Beherrschung}

Strategie als Gegenstand der Unternehmensführung ist von verschiedenen Seiten problematisiert worden (Johnson, 1987; Scholz, 1987; Chandler, 1962). Nach Mintzberg/Waters (1985, S. 257) wird Strategie als "a pattern in the stream of decisions and actions" betrachtet. Ein Schwerpunkt der Strategieforschung befaßt sich mit der Einschätzung des Prozesses und Inhalts der Strategie in verschiedenen Organisationsformen (Romme et al., 1990; Mintzberg, 1990 Schreuder et al., 1988).

Strategien betreffen vielfach allein einen bestimmten Teilbereich innerhalb der Unternehmung. Dies gilt beispielsweise für das Beschäftigungssystem. So werden interne Arbeitsmärkte vielfach als Teil der Unternehmensstrategie gesehen. Die Bedeutung der Einbeziehung der Beschäftigung in die Strategiebetrachtung betonen Bechtle (1980), Lutz (1987) und Staber/Aldrich (1988) sowie Gütermann (1989). Eine andere Forschungsrichtung, die 'labour process theory' (Edwards 1979; Loveridge/Mok 1979) hebt mehr die 'labour control' Perspektive hervor.

Das Erkenntnisinteresse konzentriert sich auf die strategische Handhabung in Bezug auf die Durchlässigkeit der äußeren Organisationsgrenzen. Die Eingangstore zum intemen Arbeitsmarkt (ports of entry) können in unterschiedlicher Form geöffnet oder geschlossen sein. Hier wird eine Überlegung, die im Organisationsrahmenkonzept vorgestellt wurde, wieder aufgenommen. Sie wird im Kapitel 8 ihren Niederschlag finden durch die gezielte Untersuchung der Präferenz interner bzw. externer Stellenbesetzung in den verschiedenen Organisationskonfigurationen.

Windolf/Hohn (1984) greifen das Konzept Webers (1972, S. 201 ff.) von der 'sozialen Schließung' auf. Gemeint damil ist der Ausschluß sozialer Gruppen von den Erwerbsmöglichkeiten. Weber spricht von Institutionen, beispielsweise Großbürokratien, Berechtigungswesen und Professionalisierung, die eine spezifische Form sozialer SchlieBung bewirken. Diese tragen dazu bei, den Austausch am Markt zu unterbinden. Soziale 
Organisierung am Arbeitsmarkt kann Markteintrittschranken (Scott, 1987; Demsetz 1989) entstehen lassen.

Windolf/Hohn (1984, S. 230 f.) führen drei Formen der Marktschließung an, die zur Organisation der Schließung am Arbeitsmarkt beitragen können.

"Die erste Form beruht auf normativer Kontrolle und hat gemeinsame Werthaltungen und Überzeugungen einer sozialen Gruppe zur Voraussetzung. ...

Die zweite Form der 'Strukturierung' beruht auf Organisationsbildung. Eine soziale Gruppe, deren Mitglieder sich in ähnlicher Interessenlage befinden, schließt sich zusammen, um Nicht-Mitglieder von Erwerbschancen auszuschließen. ... Eine der wichtigsten Formen der Strukturierung durch Organisationsbildung ist der interne Arbeitsmarkt.

Eine dritte Form der Marktschließung ist auf staatliche Organisationsleistungen angewiesen. Von praktischer Bedeutung ist sie erst, seitdem ein ausgebautes öffentliches Bildungswesen existiert, das Arbeitskräfte mit allgemein akzeptierten Zertifikaten versieht."

Die Strategien der Marktschließung (Behrens, 1984) (mit der Folge der Entstehung von Organisationen) verdichten sich in sozialen Institutionen und verstärken dadurch die Schutzfunktion der Mitglieder des intemen Arbeitsmarktes. Es gehört zu den strategischen Optionen (Rieckmann, 1989), den Austausch von Personal mit dem externen Arbeitsmarkt zu bestimmen. Schon die Wahl einer bestimmten Organisationsform kann für cinc hestimmte Beschäftigungsform einschließlich deren strategischer Beherrschung stehen. Mehr spezifisch können Rekrutierungsstrategien dazu beitragen, strategische Entscheidungen zu implementieren. Diesem Aspekt gilt im folgenden Abschnitt die Aufmerksamkeit.

\subsubsection{Rekrutierungsstrategien als Steuerung von Öffnung und SchlieBung der} Organisationsgrenze

Im folgenden gilt es, die Strategieperspektive auf die Rekrutierungsstrategie, also des Eintritts von Personen des externen Arbeitsmarktes in die Organisation zu konzentrieren (Schwan, 1992b). Diese Perspektive soll abgegrenzt werden von jener der intemen Rekrutierung, die sich ausschließlich auf Rekrutierung der Mitarbeiter richtet, die in der Organisation anwesend sind. Diese Problematik ist einem weiteren Abschnitt vorbehalten. Zunächst werden Rekrutierungsstrategien kurz vorgestellt, danach wird gefragt, welche dieser Strategien die für den intemen Arbeitsmarkt typische Rekrutierungsstrategie( $\mathrm{n}$ ) aufweisen. Geprüft wird in Kapitel 6, inwieweit die in der Realität angetroffenen Rekrutierungsstrategien typisch sind für die jeweilige Organisationskonfiguration und Übereinstimmungen feststellbar sind.

Windolf/Hohn (1984, S. 197) definieren Rekrutierungsstrategie " ... als eine Kombination verschiedener Instrumente der Personalpolitik, die den betrieblichen Verhältnissen und den lokalen Arbeitsmarktbedingungen angepaßt sind." Sie sind eingebettet in ein Netzwerk von Interessenkoalitionen, bürokratischen Entscheidungsverfahren und organisatorischen 
Maßstäben bzw. Verhandlungssystemen. Dabei spielt der Grad der Institutionalisierung von Personalpolitik und betrieblicher Interessenvertretung eine entscheidende Rolle.

Für die konkrete Rekrutierung von Personal stellt sich die Frage nach den Rekrutienungskanälen. Diese können aus sozialen Netzwerken (Betriebsangehörigen), dem Arbeitsamt, aber auch in Form von Zeitungsanzeigen bestehen. Zu den formalisienten Instrumenten der Personalselektion zählt der Gebrauch von Filtem (Bewerbungsbogen, Tests und Interviews). SchlieBlich geht es um die Festlegung der organisationsinternen Rekrutierungsmaßstäbe, die vielfach das Resultat von intemen Verhandlungen sind.

Die beiden Autoren haben in Form einer empirischen Untersuchung idealtypische Rekrutierungsstrategien entwickelt, die einen hohen Grad der Übereinstimmung mit den in der Realität angetroffenen Typen aufweisen. Die Rekrutierungsstrategien sind gekennzeichnet durch zwei Dimensionen, die das Rekrutienungsverhalten charakterisieren. Zum einen handelt es sich um 'Organisationsintelligenz' zum anderen um 'Marktmacht'. Organisationsintelligenz kennzeichnet die Art der Informationsverarbeitung und des Anpassungsverhaltens von Organisation an verändente Umweltbedingungen. 'Marktmarkt' kennzeichnet die Fähigkeit der Beherrschung und Durchdringung des Betriebes am lokalen Arbeitsmarkt. Die idealtypischen Rekrutierungsstrategien lassen sich wie folgt charakterisieren:

Abb. 9: Rekrutierungsstrategietypen

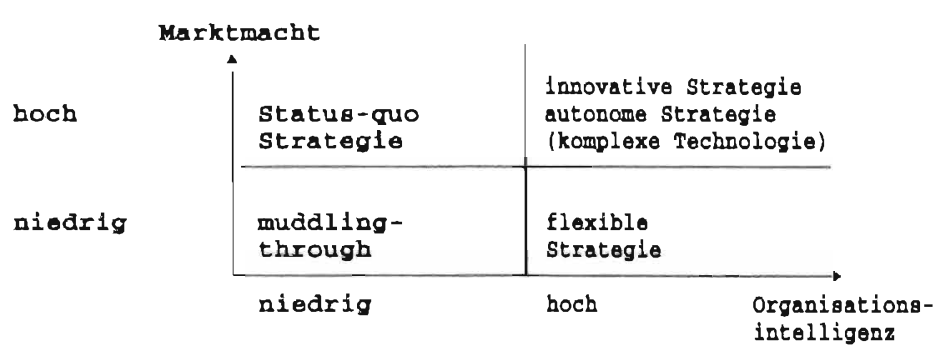

Quelle: Windolf/Hohn 1984, S. 201

Diese Übersicht zeigt im Grunde vier (fünf) unterschiedliche idealtypische Rekrutierungsstrategien. In der Realität sind Mischformen oder kombinierte Anwendungen dieser Strategien denkbar. Die Bedeutung dieser Strategien soll an Hand einer kurzen Stichwortumschreibung erläutert werden, basierend auf der tabellarischen Übersicht von Windolf/ Hohn (1984. S. 217 f.) Hierbei werden die obengenannten Strategien vorgestellt. Innovative und autonome Strategie werden getrennt voneinander erläutert, sie gehören jedoch nach der Funktionslogik obiger Abbildung in ein Feld. Unterschiedliche Felder werden der flexiblen Strategie sowie der 'muddling through'-Strategie zugeordnet, allerdings werden diese zusammen im folgenden besprochen. Dabei dienen die folgenden Kriterien als Leitfaden: Beschaffungskanäle, Auswahlverfahren, Marktposition, Idealkandidat und die Organisationeinstellung zum Risiko. 
1 Bei der 'innovativen Strategie' macht man Gebrauch von allen Beschaffungskanälen, z. B. Zeitungen, Institutionen und informelle Kanäle. Die Suchaktivitäten sind intensiv und werden damit auch kostspielig. Das Auswahlverfahren ist aufwendig. Fast alle denkbaren Filterformen kommen zur Anwendung. Unternehmen, die diese Rekrutierungsstrategie nutzen, gelten meist als Marktführer. Abgesehen von einigen vorgegebenen Auswahlkriterien existient keine genaue Beschreibung des Idealkandidaten. Das Management bestimm ausschließlich Kriterien und Kandidaten. Die innovative Rekrutienungsstrategie gilt als 'risikofreudig', d. h. man wirbt Kandidaten an, deren Verbleib im Unternehmen möglicherweise ungewiß ist.

2 Bei der 'autonomen Strategie' werden gezielt verschiedene Beschałf ungskanäle benutzt. Im Vordergrund geht es um Fachpersonal, von dem eine genaue Vorstellung des Idealkandidaten vorliegt. Die Auswahl ist in hohem Maße formalisiert. Geprüft wird der Grad der Übereinstimmung von Profil des Kandidaten und den Arbeitsplatzanforderungen. Diese Strategie setzt eine starke Marktposition voraus. Die Kriterien des Idealkandidaten liegen sehr deutlich fest. Bei der Einstellung zum Risiko läßt sich kein einheitlicher Maßstab angeben, doch versucht man, durch Insitutionalisienung des Verfahrensablaufs das Risiko weitgehend auszuschließen.

3 Bei der Status-quo-Rekrutierungsstrategie basieren die Beschaffungskanäle auf interne Arbeitsmärkte bzw. auf erweiterten intemen Arbeitsmärkten. Soziale Netzwerke können dabei auch eine Rolle spielen. Die Personalauswahl ist nicht aufwendig, vielfach reduziert auf grobe Selektion. Auch bei dieser Rekrutierungsstrategie wird eine starke Marktposition vorausgesetzt. Der Idealkandidat entstammt einer sozialen Gruppe, aus der vorwiegend rekrutiert wird. Allgemein ist man 'risiko-scheu', d. h. man vermeidet andere Rekrutierungswege einzuschlagen.

$4 / 5$ Schließlich bleibt die flexible und die muddling-through Strategie, von der letzten geben die Autoren $z u$, daß sie im Grunde genommen keine Strategie ist. Alle möglichen Beschaffungskanäle werden benutzt, sie müssen nur billig sein. Selektion ist auf ein Minimum reduziert. Betriebe, die diese Rekrutierungsstrategie praktizieren, haben in der Regel eine schwache Marktposition. Eine Beschreibung des Idealkandidaten gibt es nicht, man nimmt, was kommt. Das Unternehmen mit dieser Strategie nimmt vielfach ein hohes Risiko in Kauf. Die flexible Strategie ist dagegen von der professionellen Suche nach Personal gekennzeichnet und besteht im Rückgriff auf andere Rekrutierungstrategien.

Die zur Charakterisierung der Rekrutierungsstrategien herangezogenen Kriterien zeigen, daß eine ideallypische Zuordnung zum internen Arbeitsmarkt auf die Status-quo-Strategie zutrifft. Insbesondere bei einer größeren Beschäftigungszahl auf intemen Arbeitsmärkten bedeutet ein stärkeres Engagement der Personalabteilung (inklusiv der Professionalisierung der Personalarbeit, (Steinmann u. a., 1989; DiPrete 1987b; Pfeffer/Cohen, 1984) auch eine stärkere Inanspruchnahme der autonomen Strategie.

Die autonome Strategie läßt sich weitgehend in Verbindung bringen mit der Organisationskonfiguration der professionellen Bürokratie. 
Im übrigen zeigt sich ein relativ hohes $M a ß$ an Übereinstimmung, wenn man die den vier Organisationskonfigurationen von Mintzberg zugeschriebenen Anwerbungsmuster mit denen von Windolf/Hohn vergleicht. Der Vergleich läßt die folgende Assoziation von Organisationskonfiguration und Rekrutienungsstrategie zu. Diese Art der Zuordnung ließe sich ebenfalls auf die unterschiedlichen Formen der 'governance structures' anwenden.

Bei Maschinenbürokratien und professionellen Bürokratien sind vielfach auch Mischformen zwischen Status-quo-Strategie und autonomer Strategie festzustellen.

Tab. 2: Assoziation von Organisationskonfiguration mit Rekrutierungsstrategie (idealtypisch)

\begin{tabular}{|c|c|}
\hline $\begin{array}{l}\text { Organisationskonfiguration } \\
\text { nach Mintzberg }\end{array}$ & $\begin{array}{l}\text { Rekrutierungsstrategie } \\
\text { nach Windolf/Hohn }\end{array}$ \\
\hline Maschinenbürokratie & status-quo-Strategie \\
\hline Profeselonelle Bürokratie & Autonome Strategie \\
\hline Einfache struktur & $\begin{array}{l}\text { Flexible strategle } \\
\text { muddling through }\end{array}$ \\
\hline Adhocratie/Missionarische Form & Innovative strategie \\
\hline
\end{tabular}

Rekrutierungsstrategien zielen auf die Beherrschung und Steuerung des Zugangs zu der jeweiligen Form des Arbeitsmarktes. Über sie läßt sich der Zustrom von potentiellen Arbeitnehmem dosieren.

Jede Strategie ist charakterisiert durch eine typische Form der Offenheit bzw. Geschlossenheit was den Zugang betrifft. Hier kann allein eine erste Einsicht über die Art der Strategie und Organisationskonfiguration gewonnen werden. Quantitativ werden in der Studie von Windolf/Hohn keine Aussagen gemacht über das Verhälmis von in- und extemen Rekrutierungen. Dies erscheint insofem erforderlich, um Einsicht in die Rekrutierungsdynamik zu gewinnen, die ihren Schwerpunkt an den 'ports of entry' aber auch organisationsintern haben kann. Wie schon an anderer Stelle angedeutet, begründet diese Einsicht eine empirische Überprüfung, die in Kapitel 8 vorgesehen ist. Diese Einsicht legt darüberhinaus die Betrachtung des organisationsinternen Allokationsgeschehens nahe, die Gegenstand des folgenden Abschnitts sein wird.

\subsubsection{Konzeptionalisierung von organisationsintemen Allokationssystemen}

Interne Arbeitsmärkte stellen strukturierte Formen der Allokation von Personen auf Arbeitsplätzen dar. Auf Grund der Kenntnis der Formen intemer Rekrutierung (und deren Dominanz) auf internen Arbeitsmärkten wird es möglich, die Aufmerksamkeit zu richten auf das organisationsinterne Arbeitsmarktgeschehen. Bamey/Ouchi (1986) betonen, da B die Transaktionen von Arbeit nicht auf die Organisationsgrenzen beschänkt sind, sonderm auch innerhalb der Organisation vollzogen werden. Angeknüpft wird damit an den Arbeitsmarktstrukturdimensionen, die in Kapitel 2 vorgestellt wurden. 
In diesem Abschnitt sollen idealtypische Formen bzw. Konzepte der Allokation betrachtet werden. Dabei geht es schwerpunktmäßig um organisationsinteme 'Bewegungen' in Form von Arbeitnehmermobilität oder in Form offener Stellen. Mit Brüderl (1991) lassen sich zur Erschließung mikrostruktureller Organisationstrukturen neben den Vakanzmodellen auch Turniermodelle ansprechen. Hierauf soll kurz eingegangen werden.

Die Vakanzmodelle gehen von der Interdependenz von Karrierechancen (Preisendörfer, 1988) aus, d. h. man trägt damit sowohl den Bedingungen der Organisation Rechnung in Form freier Stellen als auch der Vergangenheit des Beschäftigten. Die Vakanzmodelle rücken von Vorstellungen ab, die allein die subjektiven Merkmale (Qualifikation etc.) berücksichtigen wie es human-capital- und status-attainment-Ansatz vorsehen.

In Whites (1970) Untersuchung kommt nicht die Mobilität, sondern die Folge offener Stellen zum Ausdruck. Untersucht wird die Entstehung und Fortsetzung von offenen Stellen über die Hierarchiestufen. Das Interesse gilt der Länge der Vakanzketten. Verglichen werden prognostizierte mit empirisch festgestellten Verteilungen. Methodisch basiert Whites Ansatz auf das Markov-Ketten-Modell (Scholz, 1989).

Als Fortentwicklung des Vakanzkettenmodells Whites sind die organisationsdemographischen Karrieremodelle zu verstehen. Ausgangspunkt ist die organisationsinteme Entstehung von freien Stellen. Damit kommt der Einfluß der Organisationsstruktur auf den individuellen Karriereverlauf zum Ausdruck. Die Arbeiten von Stewman/Konda (1983) und Stewman (1986) stehen für diesen Ansatz exemplarisch. Sie problematisieren die Vakanzstruktur in Form organisatorischer Mikrostrukturen (Brüderl, 1991). Methodische Vorgehensweise bei den organisationsdemographischen Modellen ist die Isolierung von bedeutenden Parametern der Organisationsstruktur. Die Verändung dieser Parameter impliziert Veränderungen in Bezug auf die Karrierechancen (Preisendörfer, 1987 und 1988).

Einige der Parameter sollen hier kurz aufgezeigt werden. Stewman/Konda (1983) weisen nach, daß die allgemeine Annahme einer pyramidenförmigen Anordnung von Organisationen in Bezug auf Beförderungschancen so nicht zutrifft. Vielmehr kommen die Autoren zu der Schlußfolgerung, daß die Karrierechancen sich nach der jeweiligen Besetzung in oberen Hierarchieebenen richten. Die Karrierechancen, so die Feststellung der Autoren, sind nach oben gerichtet nicht unbedingt abnehmend. Vielmehr variieren sie in Form teils offener bzw. geschlossener 'gateways' (Hondeghem, 1990).

Während die organisationsdemographischen Modelle 'Stellenumbesetzungen' auslösen beim Freiwerden einer Stelle im oberen Hierarchiebereich, gehen die Tumier- bzw. Tournamentmodelle von Rosenbaum (1979a und 1984) yom Wettbewerb um Karrierechancen innerhalb der Organisation aus. Rosenbaums Grundüberlegung basiert auf zwei Funktionen (Preisendörfer, 1987). Dabei handelt es sich um 'organisatorische Effizienz' (die richtige Person auf der richtigen Stelle) und die Gelegenheits- und Anreizstruktur, die im Beförderungssystem zum Ausdruck kommt (ein jeder soll glauben, eine Aufstiegschance zu haben). 
Das 'Rosenbaum-Modell' geht davon aus, daB die Kohorte eintretender Arbeitnehmer sich regelmäßig (ungefähr alle drei Jahre) einem Wettbewerb (Rosenbaum, 1984, 1990) unterziehen, der dann ausschlaggebend ist für die weitere Entwicklung von Mobilität in Form der Beförderung. Das Modell sieht während der Beschäftigung eine permanente Selektion vor. Damit soll die Motivation des Arbeitnehmers gefördert werden.

Rosenbaum leitet aus seinem Modell zwei Aspekte ab, die empirisch überprüft wurden (Preisendörfer, 1987). Zum einen geht es um die 'Pfadabhängigkeit', die besagt, daß die Personen bessere Karrierechancen haben, die eine bestimmte Hierarchiestufe über Hauptturniere erreicht haben im Gegensatz zu den Personen, die über Nebenpfade dieselbe Hierarchicstufe erklommen haben.

Zum anderen haben Personen, bezogen auf dieselbe Eintrittkohorte, die in einem frühen Stadium schnell befördert werden, bessere Karrieremöglichkeiten als Personen, die als erstmalige Verlierer der ersten Runde dann als Gewinner der zweiten Runde auftreten. Eine 'schnelle' Befördenung signalisiert somit 'Fähigkeiten'.

Die Wettbewerbssitutation beim Zustandekommen von Karrieremustern bietet eine neue Perspektive, die für ein dynamisches Allokationsgeschehen spricht (Rosenbaum, 1990). Gleichzeitig stellen sich aber auch Zweifel ein, wie Rosenbaum (1979b) selbst andeutet. Innerhalb der Organisation entsteht eine Art 'Sozialdarwinismus', wobei zu fragen ist, wie die Verlierer der Tumiere aufgefangen werden. Hier entsteht die Gefahr von Fluktuation und der Verlust von betriebsspezifischen Qualifikationen. Gerade auf mittlerem und gehobenem Managementniveau können Ansätze von Organisationskultur durch die 'Turnierwirkung' im Keim erstickt werden.

Schließlich soll hier ein weiterer Ansatz zur Sprache kommen. Dabei geht es um die Konzeption von DiPrete $(1987$ a, 1989). Dieser Ansatz hat intraorganisationale Mobilität zum Gegenstand, basierend auf Jobleitem als organisationsstruktureller Determinante. Ausgangspunkt ist die Abweichung von formalen Jobleitem und den tatsächlichen Mobilitätsbewegungen innerhalb der Organisation. Diese Abweichung läßı sich erklären aufgrund von Personalpolitik, fehlender Information und der Entstehung von Vakanzen innerhalb der Organisation und der Art, diese zu besetzen. Konkret wird ausgegangen von den innerorganisatorischen Grenzen bzw. deren Einfluß auf die Mobilităt. Die Berücksichtigung der organisatorischen Grenzen führt zu einer organisatorisch determinierten intemen Rekrutierungspräferenz, die bei der Besetzung offener Stellen entsteht. Die empirischen Resultate sind in Kapitel 8 zu finden.

Die Betrachtung der verschiedenartigen Modelle intemer Allokation (Soerensen, 1983) legt die folgende Schlußfolgerung nahe. Die hier aufgezeigten Ansätze mit Ausnahme des letzten werden den Strukturdimensionen des intemen Arbeitsmarktes nur unzureichend gerecht. Im nächsten Schritt wird deshalb die Konzeption DiPretes mit den Arbeitsmarktstrukturperspektiven konzeptionell verbunden. Auf diese Weise werden Defizite der unterschiedlichen Konzeptionen ausgeschaltet. Außerdem ermöglicht die Integration der Konzepte eine flăchendeckende Sichtweise von ganzen Organisationen bzw. Organisationskonfigurationen. Kemstück der Allokation sind 'Einheiten' (units). Grenzen innerhalb der Organisation beschränken die Allokation in Form von Arbeitnehmermobilität. Der Entwurf 
einer solchen Konzeption ist Gegenstand des folgenden Abschnitts. Er beinhaltet eine Brückenfunktion zwischen den vorher entwickelten Arbeitsmarktstrukturdimensionen und den mehr organisationstheoretisch geprägten Aspekten.

\subsubsection{Konzeptionalisisierung organisatorischer Allokationsgrenzen}

Die in der analytischen Erschließung von Arbeitsmarktstrukturebenen von Doeringer/Piore (Kapitel 2) eröffneten Perspektiven bilden die Grundlage für die Konzeptionalisierung organisatorischer Grenzen, die die Arbeitnehmermobilität organisationsintern steuem bzw. einschränken. Die Arbeitsmarktstrukturebenen bilden ein Steuerungs- und Allokationsinstrument, das organisationsintern zur Allokationsflexibilität durch abgegrenzte Jobterritorien beitragen kann. Bei internen Arbeitsmärkten steht 'social boundary management" (Feijen, 1990) im Vordergnund. Dies bedeutet, den Grad an Offenheit bzw. Geschlossenheit intemer organisatorischer Grenzen zu bestimmen.

Hier werden Überlegungen wieder aufgenommen, die mit dem Organisationsrahmenkonzept am Anfang dieses Kapitels entwickelt wurden. Dabei steht die Frage im Vordergrund, wer kann als Person welche organisatorischen Grenzen überwinden und entwickelt dabei welches Mobilitätsprofil. In Bezug auf inteme Arbeitsmärkte geht es um:

"... mechanisms for bringing employees more fully and firmly within the boundaries of an organization ..." (Scott, 1987, p. 178).

Wir gehen nun im folgenden auf die 'boundaries' ein.

Die erste 'Hürde' ist die äußere Organisationsgrenze. Zugang zur Organisation finden nur solche Personen, die den Rekrutierungsnormen entsprechen. Organisationsintem sind weitere mobilitätsbeschränkende Grenzen anzutreffen, die die Mobilitätschancen strukturieren. Die Durchlässigkeit der organisatorischen Grenzen hängt ebenfalls ab von den Personalstrategien in den verschiedenen Facetten, die von 'labor control' (Edwards, 1979) bis hin zu bürokratischen (institutionalisierten) Regeln (Halaby, 1978; Grandjean, 1981; Stark, 1986) reichen.

Die Konzeptionalisierung der organisatorischen Grenzen setzt bei Mintzberg $(1979,1989)$ ein. Im folgenden sollen die Grenzen konzeptionalisiert werden, ausgehend von der äußeren Organisationsgrenze bis hin zu den mikroorganisatorischen Teilgrenzen. Hierbei wird die Arbeitsmarktperspektive integriert.

Die äußere Grenze trennt die Umgebung von der Organisation. Es handelt sich ebenfalls um die Grenze zwischen in- und externem Arbeitsmarkt. Im Vordergrund steht die Organisationskonfiguration der Maschinenbürokratien und der professionellen Bürokratien. Beide Organisationskonfigurationen unterscheiden sich deutlich durch Aufbau und Anlage. Diese Besonderheit der jeweiligen Konfiguration bestinumt die Art des Überschreitens (boundary crossing) der äußeren Organisationsgrenzen für Personen, die von außen Zugang zur Organisation erhalten. Konkretisieren läßt sich der Zugang durch die 'ports of entry' und deren Verteilung über die Hierarchieniveaus. Die folgende Abbildung vermittelt eine Übersicht unterschiedlicher Formen organisatorischer Mobilitätsgrenzen. 
Abb. 10: Formen von Mobilitätsgrenzen nach Mintzberg, DiPrete und anderen

\begin{tabular}{|c|c|c|}
\hline Grenzen & |Organisationskriterium & Autor \\
\hline \multicolumn{3}{|c|}{ Dominiert von der theoretischen Perspektive Mintzbergs } \\
\hline $\begin{array}{l}\text { externe Grenze } \\
\text { interorg. Niveau }\end{array}$ & $\begin{array}{l}\text { Organisationskonfiguration } \\
\text { in-/externe Arbeitsmarkt }\end{array}$ & $\begin{array}{l}\text { Mintzberg } \\
\text { Doeringer/Ptore }\end{array}$ \\
\hline intraorg. Niv. A & $\begin{array}{l}\text { parta } \\
\text { Segmente } \\
\text { Arbeitgmäkte Innerhalb der } \\
\text { Organisation } \\
\text { governance structure }\end{array}$ & $\begin{array}{l}\text { Mintzberg } \\
\text { Sengenberger } \\
\text { Osterman, Alt- } \\
\text { hauser/Ralleb. } \\
\text { Williamson }\end{array}$ \\
\hline \multicolumn{3}{|c|}{ Dominlert von der theoretischen Perspektive DiPretes } \\
\hline Intraorg. N1v. B & Abteilung & Diprete \\
\hline Intraorg. Niv. C & Jobleiter & $\begin{array}{l}\text { Baron et al., } \\
\text { Diprete }\end{array}$ \\
\hline intraorg. Niv. D & Ber & Diprete \\
\hline
\end{tabular}

Erklärung: Niv.=Niveau; intraorg.=intraorganisational; interorg.=interorganisational;

Neben den Organisationskonfigurationen mit der jeweiligen äußeren Grenze erweist sich die Aufteilung in 'parts' und die daraus resultierende Grenzziehung als eine grobe Form der Differenzierung der 'Organisationsteile', die die Mobilität deutlich strukturiert. In obiger Abbildung wird diese Art der intraorganisationalen Grenzziehung als Niveau A bezeichnet. Äquivalente mit mehr 'Arbeitsmarktorientierung' sind Segmente sowie die verschiedenen Arten von Arbeitsmärkten innerhalb einer Organisation bzw. 'govemance structure'.

Bei den folgenden 'Grenzniveaus' dominiert die theoretische Perspektive von DiPrete aufgrund einer ansprechenden mikroorganisationalen konzeptionellen Unterbauung. Sie bestehen aus der 'Abteilung' oder dem intraorganisatorischen Niveau B. Jobleiter repräsentieren die nächst kleinere Organisationsgrenze (intraorganisatorisches Niveau C), gefolgt von einem weiteren Grenztyp, nämlich der der Berufsgruppe (intraorganisatorisches Niveau D).

Die Konzeption allokationsrelevanter Organisationsgrenzen wird im Rahmen der empirischen Untersuchung berücksichtigt. Dieser Ansatz ist kompatibel vor allem hinsichtlich der analytischen Durchdringung mit dem Organisationsrahmenkonzept (Scott, 1987). Die Organisationsstrukturen gewinnen jedoch nur dann Bedeutung für die Allokation, wenn sie an intraorganisationaler Mobilitäl gekoppelt sind. 


\subsection{Konzeptionalisierung der Mobilität auf dem internen Arbeitsmarkt}

An dieser Stelle wird die im oberen Teil dieses Kapitels, Abschnitt 3.4.2 angesprochene Forderung nach einem auf die integrative Sicht der Infrastruktur intemer Arbeitsmärkte abzielenden Konzeptes eingelöst. Die konzeptionelle Erarbeitung steht im Mittelpunkt dieses Abschnitts.

Das Konzept berücksichtigt die typischen Merkmale interner Arbeitsmärkte wie Funktionsketten, 'ports of entry' sowie Arbeitnehmermobilität und andere. Zunächst gilt die Aufmerksamkeit der Mobilität der Beschäftigten.

\subsubsection{Allgemeine Vorstellungen zur Konzeptionalisierung von Mobilität}

Mobilitätsprozesse sind immer wieder Gegenstand der wissenschaftichen Auseinandersetzung gewesen. Inspiriert von den Ideen der 'status-attainment-Theorie' und der Humankapitaltheorie sind in der Vergangenheit zahlreiche Untersuchungen (Blau/ Ducan, 1967; Rosenbaum, 1984; Spenner/Otto/Call, 1982; Hachen, 1990) vor diesem Hintergrund auf nationalem Niveau erfolgt. Preisendörfer (1988) kritisiert, daß in diesen Ansätzen vorwiegend individuelle Determinanten den sozialen Status bestimmen und weniger strukturelle Kontextfaktoren, die aus den "... historischen, gesamtgesellschaftlichen und organisationalen Rahmenbedingungen für individuelle berufliche Mobilität..." abzuleiten sind (Preisendörfer, 1988, S. 690). Undeutlich bleiben die Mechanismen und Prozesse, die dazu führen, individuelle soziale Merkmale in benflichem Erfolg umzusetzen.

Ein anderer Zweig der Mobilitätsforschung ist die aus dem dualen Arbeitsmarktkonzept resultierende Segmentationsforschung. Ziel war es, Diskriminierungen am Arbeitsmarkt zu erschließen. Bei der Neuorientierung der Mobilitätsforschung rückt als Verarbeitung der oben geäußerten Kritik die 'Strukturebene' in den Vordergrund. Gemeint ist eine Orientierung auf Betriebe und Organisationen. Mit Preisendörfer (1988, S.692) lassen sich folgende Argumente für diese Orientierung ableiten:

"(l) Arbeitsverhältnisse mit langfristiger Bindung an einen Betrieb sind in den Berufsverläufen von Individuen faktisch dominierend (empirisches Argument).

(2) Betriebe und Organisationen geben den unmittelbaren Kontext ab, in dem sich Mobilitätsprozesse vollziehen; gleichzeitig lassen sich Betriebe und Organisationen als eigenständig handelnde Akteure mit bestimmten Zielen und Interessen konzipieren (konzeptionelles Argument).

(3) Im Erfahrungs- und Erlebnishorizont der Individuen vollzieht sich berufliche Mobilität zumeist in der Form von Aufstiegs- und Abstiegsprozessen auf betrieblichen Hierarchieskalen (alltagspraktisches Argument)."

Ausdruck dieser Neuorientierung in der Mobilitätsforschung sind die Arbeiten von Rosenbaum (1984) und Köhler/Preisendörfer (1989) sowie Brüderl (1991). Eine weitere Quelle 
der Mobilitäıfforschung ist die 'Karriereforschung', deren Wurzeln in den Vereinigten Staaten zu finden sind. Auch hier finden sich zahlreiche Beiträge zur 'intraorganisationalen Mobilität'. Dazu rechnen mit einer Akzentuienung von Technologien die Arbeit von Vardi/Hammer (1977). Außerdem die Arbeiten, bei denen Karrieremuster Gegenstand der Untersuchung sind (Scholl, 1983; Anderson/Milkovich/Tsui, 1981; Gerpott, 1988).

Mit dem internen Arbeitsmarkt verbindet sich das Mobilitätskettenkonzept, auf das im folgenden näher eingegangen wird. Nachteil bei der Mobilitätsforschung ist, daß vielfach nur bestimmte Mobilitätsmuster in den Vordergnund rücken, bei denen die Form und Spezifität der Organisation als Ganzes aus den Blickwinkeln gerät.

\subsubsection{Das Mobilitätskettenkonzept}

Das Mobilitätskettenkonzept ist ein Bestandteil des internen Arbeitsmarktes. Mobilitätsketten (synonym auch Jobleiter genannt) bilden die organisatorische Infrastruktur intemer Arbeitsmärkte. Die inhaltliche Auseinandersetzung mit dem Mobilitätkettenkonzept soll dazu beitragen, jenseits von Definitionen die konzeptionellen Grundlagen des Konzepts einzuschätzen. Gleichzeitig soll der Zugang zur Empirie erschlossen werden.

Die Allokationsstruktur interner Arbeitsmärkte besteht nach Doeringer/Piore (1971, S. 50) aus 'internal mobility clusters', die bestehen aus: "...groupings of jobs within which an employee is customarily upgraded, downgraded, transferred and laid off." Die Arbeitsplätze innerhalb dieser 'mobility cluster' basieren auf folgenden Elementen:

I aufeinander bezzogene Fähigkeiten und Arbeitserfahrung,

2 ähnliche Niveaus bezüglich der Arbeitsinhalte und

3 eine gemeinsame funktionale oder abteilungsorientierte Organisation, in der mehrere Funktionen gruppiert sind, sowie

4 einen Arbeitsschwerpunkt.

Mobilitätscluster variieren in Abhängigkeit von der Art der Mobilität. Diese umfaßt alle denkbaren Möglichkeiten horizontaler und vertikaler Mobilität, wie zum Beispiel: Beförderung, Entlassung und Seitenwechsel. Entsprechend dem pyramidalen Charakter der Organisation, nehmen die Möglichkeiten intemer Mobilität nach oben hin ab und nach unten $\mathrm{zu}$.

Je nach Schwerpunkt der Untersuchung werden in der Literatur mehrere Synonyme benutzt. So werden folgende Begriffe nebeneinander gebraucht: Mobilitätskette, Jobleiter, Funktionskette und Betörderungsleiter sowie Karriereleiter.

Während bei Doeringer/Piore der Begriff 'mobility cluster' in ihrem Hauptwerk (1971) dominiert, erfährt die Mobilitätskette in einem Artikel Piores (1978) eine weitere konzeptionelle Unterbauung. Dies erfolgte vor dem Hintergnund der Suche nach einer Erklärung von Mobilität innerhalb und zwischen den Arbeitsmarktsegmenten. Die Mobilitäts- 
kette besteht nach Piore (1978) aus einer Abfolge von Positionen (Stationen), die nicht nur bestimmte Arbeitsinhalte umfassen sondern auch ein 'Stiuck' Sozialisation des Arbeitnehmers. Bei den Mobilitätsketten handelt es sich um Aufstiegslinien, die vielfach mit Gewerkschaften ausgehandelt werden und Institutionalisierung erfahren in Form von Kollektivverträgen. Mobilitătsketten haben eine unterschiedliche Länge. Darin liegt eine Beschränkung, die häufig gekoppelt ist an organisatorische Mobilitätsgrenzen.

Ein weiterer Aspekt der Funktion der Mobilitätskette besteht in Form eines automatischen beiläufigen Lemens. Damit sollen produktive Eigenschaften bei Arbeitnehmern auf kostengünstige Art befördert werden. Mobilitätsketten führen zur Strukturierung von Arbeitskräften und Arbeitsplätzen (Singer, 1986). Sie sind damit Gegenstand betrieblicher Strategien hinsichtlich der Verwertung betrieblicher Qualifikationen (Drumm, 1989a; Becker, 1985).

Eine empirische Analyse von Mobilitätsketten in den Vereinigten Staaten ist Gegenstand der Arbeit von Baron u. a. (1986). Neben der Betrachtung der Mobilitätsketten steht der Bezug zum intemen Arbeitsmarkt in der Untersuchung im Vordergrund. Ein Ergebnis der Untersuchung ist die Unterschiedlichkeit von Arbeitsmarktstrukturen, die sich nicht einer 'mechanischen' Auffassung von der Dualität oder Segmentation des Arbeitsmarktes (Baron u. a. , 1986) zuordnen lassen sondern den konkreten organisatorischen und 'suborganizational' Bestimmungsfaktoren (Scott, 1987) der Beförderung zurechnen lassen.

Im folgenden soll kurz auf einige Ergebnisse der Untersuchung eingegangen werden. $\mathrm{Zu}$ den Erkenntnissen gehört, daß die Unternehmensgröße einen positiven Einfluß auf die Anwesenheit von Jobleitem hat. Die Autoren (Baron u. a., 1986) zeigen auf, daß Personen, die einen bestimmten Rang auf Jobleitem einnehmen, auf der Basis betriebspezifischer Qualifikation auch zukünftig Beförderungschancen haben. Interessant ist auch die Aussage über berufliche Differenzen in Jobleitern. Professionelle und technische Arbeitsplätze sind seltener in Jobleitern anzutreffen als Arbeitsplätze in der Produktion. In den Fällen, in denen professionelle und technische Mitarbeiter in Jobleiterm angetroffen werden, befinden sie sich auf 'unterem Niveau'. Die Autoren sehen darin eine Bestätigung, daß Angestelltenarbeitsplätze leichter von außen zugänglich sind auf Grund der Übertragbarkeit der Qualifikation.

Die Existenz von Schlüsselarbeitsplätzen auf Jobleitem wird dann mehr angetroffen, wenn sich viele Jobs auf der Leiter befinden. Unter den Arbeitsplätzen, die auf einer Jobleiter anzutreffen sind, gibt es deutliche geschlechtsspezifische Unterschiede (DiPrete/Soule, 1988).

Aufschlußreich sind die Erkenntnisse der Untersuchung hinsichtlich der Arbeitsplätze, die umfangreiche Fähigkeiten zur Informationsverarbeitung beinhalten. Danach sind jene Jobs weniger in Jobleitem anzutreffen, die diesen Kriterien entsprechen. Soweit diese Arbeitsplätze dennoch auf Jobleitern zu finden sind, so handelt es sich um Stelleninhaber, die an gehobener Position auf der Jobleiter stehen. Technischen Spezialisten wird der Zugang zur Organisation auf dem Wege der externen Rekrutienung ermöglicht. Die Autoren sehen darin eine Bestätigung für die wechselnde Inanspruchnahme von in- und externen Arbeitsmärkten als Möglichkeit der Rekrutierung. 
Baron u. a. (1986) weisen nach, daB Jobleitem verschiedene berufliche Kategorien umfassen, d. h. es ist für Männer (nicht für Frauen) möglich. die beruflichen Grenzen verschiedener Berufskategorien durch Mobilität zu überwinden mit dem Ziel, einen höheren Status zu erreichen. Selbst auf Jobleitem, auf denen sich sowohl Frauen als auch Männer befinden, genießen Männer deutlich den Vorrang bei der Beförderung (DiPrete/ Soule, 1988; Kanter, 1977; v. d. Burg, 1992).

Die Untersuchungsresultate heben die Rolle der Jobleitem hervor und bestätigen die enge Koppelung zu intemen Arbeitsmärkten. Die Jobleitem erzeugen eine bisher nicht erkannte Komplexität innerhaJb der Arbeitsorganisationsanalyse. Dies führt zu der Konsequenz, in Kapitel 6 allein exemplarisch auf bestimmte Funktionsketten einzugehen.

\subsubsection{Theoriebezogenenes Infrastrukturkonzept interner Arbeitsmärkte}

Eine weitere Operationalisienung eines Infrastrukturkonzeptes intemer Arbeitsmärkte nach der Transaktionskostentheorie hinsichtlich der 'governance structures' ist aus der Literatur nicht abzuleiten. Das Interesse konzentriert sich deshalb auf die für inteme Arbeitsmärkte modifizierte Übersicht Mintzbergs.

Mintzberg hat die entwickelten Organisationskonfigurationen mit Kriterien versehen, die die eine von der anderen Organisationsform unterscheidbar machen. Die von Mintzberg entwickelten Organisationsdimensionen sind von allgemeiner Art. Im Rahmen dieser Untersuchung wurden diese Dimensionen auf die Infrastruktur intemer Arbeitsmärkte abgewandelt. Diese Kriterien (Mintzberg, 1979, S. 466 f.; 1983, S. 280 f.) erleichtern den empirischen Zugang. Im Rahmen dieser Arbeit bilden diese Kriterien, zugeschnitten auf den internen Arbeitsmarkt, die Grundlage für die Einschätzung organisationsspezifischer Beschäftigungskennzeichen. Sämtliche Kriterien werden im folgenden auf die Bedeutungsinhalte der Infrastruktur interner Arbeitsmärkte hin 'übersetzt' bzw. abgeleitet. Die Übersicht sieht wie folgt aus: 
Tab. 3: Infrastrukturkonzept intemer Arbeitsmärkte für Maschinen- und professioneller Bürokratie

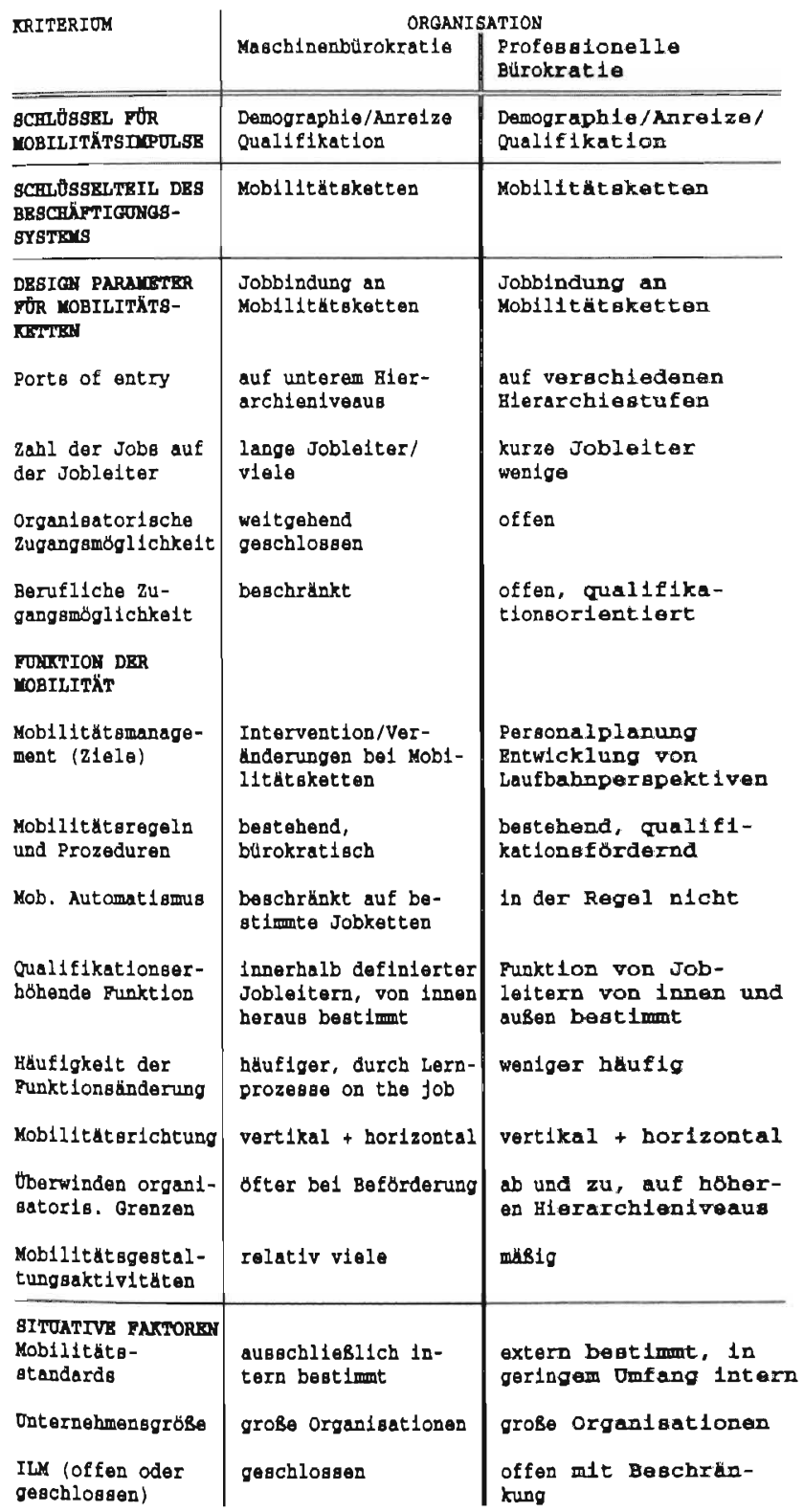

Die fettgedruckten Kriterien repräsentieren Überbegriffe. 
Die Ausprägung von Beschäftigungskennzeichen bei den beiden Organisationskonfigurationen zeigt ähnliche aber vielfach auch gegensätzliche Kriterien. Mit dieser Übersicht liegt ein ins Detail gehendes Konzept vor, das zur Formulierung von Hypothesen den Hintergrund bildet und damit eine Brückenfunktion bildet für die empirische Prüfung. Ergänzt und vervollständigt werden damit die allgemeinen und beschäftigungsrelevanten Aussagen, die aus der Literatur für die jeweilige Organisationskonfiguration abgeleitet wurden.

\subsection{Konzeptionalisierung der Besetzung offener Stellen}

Rekrutierungsstrategien zielen auf Personalverändenung in der Arbeitsorganisation von innen als auch von außen. Mobilität ist dabei eine Ausdrucksform der Umsetzung von Beschättigungsstrategie. Eine weitere Ausdrucksform besteht in der Besetzung offener Stellen. Auch hier stellt sich eine Wahlmöglichkeit in Bezug auf inteme Arbeitsmärkte bei der Besetzung von außen und/oder innen ein. Unter welchen Bedingungen wird die eine oder andere Lösung angestrebt und was sind die Einflußgrößen für eine bestimmte Lösung? Lassen sich für den internen Arbeitsmarkt typische Besetzungsmuster ableiten? Die Fragestellung legt eine Aufteilung in 'Rekrutierung von außen' und 'Rekrutierung von innen' nahe.

\subsubsection{Die Besetzung offener Stellen von außen}

Auf internen Arbeitmärkten ist in der Regel eine inteme Mobilitätsdynamik vorhanden. Es ist daher naheliegend, in erster Linie inteme Kandidaten für die Besetzung offener Stellen zu suchen. Erst die Erschöpfung des internen Kandidatenreservoirs legt eine Rekrutierung von außen nahe. Ein weiterer Grund ist darin zu sehen, daß aus demographischen Gründen kontinuierlich Personen als 'Nachwuchs' eingestellt werden. Damit wird eine Beschäftigungsstabilität erreicht, die Kontinuität ermöglicht.

\subsubsection{Die interne Besetzung freiwerdender Stellen}

In diesem Abschnitt steht die Stellenbesetzung über den internen Arbeitsmarkt zentral. Die Methodologie basien im Wesen auf der von DiPrete $(1987$ a, 1989) vorgenommenen empirischen Untersuchung. Die DiPrete-Analyse geht von der Dominanz interner Mobilität als Grundlage für die Besetzung offener Stellen aus. Als Grundidee liegt die Annahme zugrunde, daß Beschäftigungs- und Organisationseigenheiten dahingehend wirken, daß die Unterschiedlichkeit zwischen formalen Jobleitern und verhaltensorientiertem Karrieremuster abnimmt bzw. sich angleicht. Demgegenüber steht, da $B$ auch Eigenschaften existieren, die auf das Gegenteil hinwirken, d. h. daß die Unterschiedlichkeil weiter akzentuiert wird. In der von DiPrete $(1987 a, 1989)$ durchgeführten Mobilitätsanalyse nimmt die Existenz von Jobleitem eine zentrale Bedeutung ein, da diese die Mobilitätschancen von Beschäftigten strukturieren.

Mobilitätschancen werden dadurch eingeschränkt, daß organisatorische Grenzen für die Mobilität bestehen (Scott, 1987). Diese können abhängen von den Grenzen der Organisation selbst, aber auch durch die Beschränkung der Mobilität auf einer Jobleiter und durch Abteilungsgrenzen innerhalb der Organisation. Auch wenn diese Grenzen weniger eine 
formale Rolle innerhalb der Personalpolitik spielen, so haben sie doch deutlichen EinfluB auf die Strukturierung organisationsinterner Mobilität. Hinzu kommt, daß bei der potentiellen Besetzung einer freien Stelle innerhalb der Organisation beim Arbeitnehmer nicht die Anforderungen der Stelle bekannt sind und vielfach auch die Tatsache, daß eine Stelle intern zu besetzen ist. Die Abwesenheit vollständiger Information bedeutet eine Kanalisierung der Mobilität über organisatorisch und beschäftigungspolitisch festgelegte Jobleitern.

Die Durchlässigkeit organisatorischer und beschäftigungspolitischer Grenzen kann auf vielerlei Weise erreicht werden. Die Überwindung der Grenzen kann erfolgen aufgrund von qualifikatorischen oder informationsbezogenen Möglichkeiten sowie aus der besonderen Anordnung der Arbeitsplätze und aus dem Maß der Institutionalisierung der formalen Struktur. In diesen Möglichkeiten der Überwindung der Grenzen liegen sowohl die Chancen als auch die Risiken, zu einer Übereinstimmung von formaler und verhaltensorientierter Struktur zu kommen. Die formale Arbeitsplatzstruktur ist meistens in Arbeitsplatz-bzw. in Arbeitsinhaltsbeschreibung schriftlich fixiert. Daneben kann man aufgrund betrieblicher und persönlicher Notwendigkeiten davon abweichen, d. h. das aktuelle Verhalten entspricht nicht mehr der formell fixierten Stelle. DiPrete nennt Aufgabenunbestimmtheit (task idiosyncracy) (Miner, 1987) sowie die Wirkungen der unvollständigen Information, die die Übereinstimmung zwischen formaler und verhaltensorientierter Struktur erleichtern.

Fehlende Übereinstimmung kann dadurch entstehen, daß die Jobleiter zu breit besetzt ist. Damit wird die potentielle Auswahl von Personal erschwert. AuBerdem kann die Jobleiter als Rekrutierungsquelle ihre Wirkung verlieren, wenn zu oft aus dieser Quelle rekrutiert wird und die Chance des 'Nachwachsens' von Personal nicht enmöglicht wird. Daneben tritt eine fehlende Übereinstimmung auf als Folge von folgenden organisatorischen Effekten. Dazu rechnet (DiPrete, 1987a):

- die pyramidale Gestalt der Organisation,

- das Erreichen der höchsten Funktionsstufe innerhalb der Jobleiter.

- die abteilungsübergreifende Jobplazierung zum Zwecke des Erwerbs von Erfahrung bei Managern.

Untersucht wurden vier Büros der amerikanischen Regierungsverwaltung im Hinblick auf die potentielle Besetzung von Vakanzen (DiPrete, 1989). Im Wesen basiert die in dieser Untersuchung benutzte Methodologie auf der DiPretes.

Einige Unterschiede zwischen der DiPrete-Untersuchung und der hier vorliegenden Untersuchung liegen in der Tatsache, daß diese auf vier verschiedenen autonomen Organisationen basiert, während die DiPretes vier Büros zum Gegenstand hat, die alle Teil einer größeren Organisation sind. Die organisatorische und beschäftigungspolitische Heterogenität unserer Untersuchung ist im Vergleich mit der DiPrete-Untersuchung größer. Während DiPrete allein ausschnittsweise höhere Funktionsniveaus in die Untersuchung einbezieht, wird in der vorliegenden Untersuchung dem vollen Funktionsspektrum von Organisationen Rechnung getragen. Mit Bezug auf den intemen Arbeitsmarkt wird damit ein vollständigeres Bild entwickelt, was eine bessere Einschätzung des Organisations- (Allokations-) geschehens ermöglicht. 
Dies gilt vor allem für die Allokationsform 'Jobleiter' und setzt sich for in der Beurteilung des Segmentationsphänomens intemer Arbeitsmärkte.

Verschiedene Prioritäten einer Stellenbesetzung aus Funktionskette, Abteilung und Berufsgruppe, die ebenfalls die konzeptionierten Organisationsgrenzen berücksichtigen, wurden zuvor festgelegt. Im Vordergrund steht die Idee, daß eine Stellenbesetzung in erster Linie aus der "nächsten Umgebung", d. h. aus derselben Abteilung und derselben Funktionskette besetzt wird. Soweit in dieser Kategorie ein Mangel an geeigneten Kandidaten besteht, wird bei der 'Erschließung' potentieller Kandidaten die nächste Kategorie angegangen. Dies kann beim Abarbeiten verschiedener Prioritäten schließlich zu einer organisationsweiten Suche nach internen Kandidaten führen. Das von DiPrete entwickelte Modell soll hinsichtlich der Besetzung offener Stellen im Rahmen der empirischen Untersuchung Anwendung finden, da es Aufschluß bietet über die Allokation auf intemen Arbeitsmärkten.

\subsection{Zusammenfassung und Schlußfolgerungen}

Dieses Kapitel hat gezeigt, daß Organisations- bzw. Arbeitsmarkttheorie in einem hohen Grad kompatibel sind. Das Fundament der Organisationsrahmentheorie wurde ausgefültt durch die beiden Organisationstheorien, der Transaktionskostentheorie und der Kontingenztheorie. Bei der Transaktionskostentheorie konzentrierte sich die 'Ausfüllung' auf die Abgrenzung und ldentifikation der 'govemance structure', die im Kontext intemer Arbeitsmärkte und den damit verbundenen Beschäftigungsmerkmalen Relevanz besitzt. Bei der Kontingenztheorie wurden aus Mintzbergs Organisationskonfigurationen jene selektiert, die Ähnlichkeit mit den Beschäftigungsmerkmalen intemer Arbeitsmärkte aufweisen. Ein Vergleich der jeweils aus der Theorie abgeleiteten 'Organisationsformen' des intemen Arbeitsmarktes zeigt vergleichbare Züge auf. In einem weiteren Schritt wurde auf der Basis der Theorie für die jeweiligen Organisationskonfigurationen allokationsrelevante Organisationsgrenzen und ein auf die Infrastruktur intemer Arbeitsmärkte abzielendes Konzept entwickelt.

Inteme Arbeitsmärkte zeugen qua Komplexität bzw. Unterschiedlichkeit der Determinanten von der Rolle intemer Arbeitsmärkte als Sammelbegriff für unterschiedliche Theorieströmungen. Mit Althauser/Kalleberg (1990, p. 322) läBt sich eine Erfahrung wiedergeben, die aus einer Bilanz dieser Autoren abzuleiten ist:

"The concept (of internal labor market, R. S.) has numerous ideas and key images; its conceptualization depends not only on the purpose of research or the level of analysis (e. g., institutional vs. individual) but on the academic discipline of the writer."

Unsere Akzentsetzung erfolgt durch die spezifische Ableitung der Rolle intemer Arbeitsmärkte im jeweiligen Theoriekontext.

Die Organisationstheorien geben den Hintergrund ab für die Entwicklung spezifischer Mobilitätskonzeptionen (Siedenbiedel, 1984) sowie einer Konzeption zur Besetzung offener Stellen auf dem internen Arbeitsmarkt. Hierin liegt der Ausgangspunkt für eine empirische Umsetzung der zentralen Fragestellung. 


\section{$4 \quad$ Zentrale Fragestellung und erkenntnisleitende Hypothesen der Untersuchung}

Kapite] 2-3 bilden das theoretische Fundament dieser Arbeit. Kapitel 4-5 sind als Überleitungskapitel zu verstehen, um den Weg von der Theorie zur Empirie zu ebnen. In diesem Abschnitt wird kurz noch einmal auf die zentrale Fragestellung der Untersuchung eingegangen. Daneben werden Untersuchungshypothesen aufgestellt, die die zentrale Fragestellung hinsichtlich einer empirischen Überprüfung operationalisieren. Außerdem werden jene Informationen erschlossen, mit deren Hilfe die Untersuchungshypothesen verifiziert bzw. falsifiziert werden. Kapitel 6-8 sind den Ergebnissen der empirischen Untersuchung gewidmet.

\subsection{Erkenntnisleitende Fragestellung}

Im theoretischen Teil der Arbeit wurde durch Ableitung die Konkretisierung des Untersuchungsgegenstandes vorgenommen. Die daraus gewonnenen Einsichten lassen sich zu der in Kapitel 1 vorgestellten zentralen Fragestellung der Untersuchung verdichten, die hier wiederholt wird:

In welchem Maß unterscheiden sich Maschinenbürokratien und professionelle Bürokratien in der Prägnanz der Anwesenheit der angegebenen Beschäftigungskennzeichen von internen Arbeitsmärkten?

Im folgenden sollen die Ausführungen in den vorangegangenen Kapiteln im Hinblick auf die zentrale Fragestellung zusammengefaßt werden. In der zentralen Fragestellung richtet sich das Erkenntnisinteresse auf den intemen Arbeitsmarkt und seiner spezifischen Ausprägung von Beschäftigungsmerkmalen. Die ersten Schritte der Konkretisierung der zentralen Fragestellung richteten sich auf die Ermittlung dicser Beschäftigungskennzeichen. Aufgrund der Komplexität intemer Arbeitsmärkte wurde ein auf die Arbeitsmarktstrukturdimensionen zielendes Erschließungskonzept gewählt, das systematisch die Arbeitsmarktstrukturdimensionen erkennen läßt. Dabei diente die Theorie interner Arbeitsmärkte, so wie sie von Doeringer/Piore vertreten wird, als Ausgangspunkt. Auf der Basis der analytischen Strukturen interner Arbeitsmärkte konnten unter Heranziehung aller relevanten Konstrukte kein in sich logisch konsistentes Bild abgeleitet werden. Die Arbeitsmarktstrukturdimensionen repräsentieren einen heuristischen Wert. Herausgearbeitet wurden Kriterien, die eine systematische Sicht unterschiedlich segmentierter Arbeitsmarktstrukturen bieten.

Als weiterer Schritt der Operationalisierung der zentralen Fragestellung wurde aus zwei Organisationstheorien, der Transaktionskosten- und der Kontingenztheorie, beschäfti- 
gungstypische Kennzeichen abgeleitet. Konkretisiert wurde dies in den 'governance structures' als Ausfluß der Transaktionskostentheorie und in den Organisationskonfigurationen als Ausdruck der Kontingenztheorie. Bei der letzten Theorie war die Erschließung der Beschäftigungskennzeichen dadurch geprägt, daß die allgemeinen Kriterien zur Erschließung von Organisationen Anhaltspunkte boten für die Ableitung beschäftigungsspezifischer Kennzeichen. Bei den 'governance structures' ließen sich die Beschäftigungskennzeichen spezifizieren, jedoch auf relativ abstraktem Niveau.

Als Konsequenz wurde den beschäftigungsbezogenen Kennzeichen der Organisationskonfigurationen eine höhere Gewichtung zugeschrieben. Dazu waren zwei voneinander unabhängige Schritte einzuleiten. Einerseits wurden allgemeine beschäftigungsrelevante Kriterien aus der Literatur abgeleitet und synoptisch (Tab. 1) präsentiert. Diese beschäftigungsrelevanten Kennzeichen sind von der jeweiligen Organisationskonfiguration geprägt, d. h. es werden in diesem Stadium nur solche Merkmale berücksichtigt. Bislang ausgeklammert waren die Beschäftigungskennzeichen interner Arbeitsmärkte. Um diesen Anspruch einzulösen, wurde andererseits ein Infrastruknurkonzept intemer Arbeitsmärkte für Maschinen- und professioneller Bürokratie entwickelt (Tab. 3). Damit stehen in systematisierter Weise Beschäftigungskennzeichen zur Verfügung, die typisch sind für die Organisationskonfigurationen, jedoch auf die Komplexität intemer Arbeitsmärkte abstellen.

Die Beschäftigungskennzeichen ermöglichen ein differenziertes Bild der Infrastruktur interner Arbeitsmärkte. Im Rahmen der empirischen Untersuchung soll geprüft werden, ob Elemente dieser Infrastruktur von Beschäftigungskennzeichen in ihrer Ausprägung in der Realität anzutreffen sind. Damit gilt es, exemplarisch den Nachweis einer unterschiedlichen Struktur interner Arbeitsmärkte in Maschinen- und professionellen Bürokratien zu erbringen.

Die zu bildenden Hypothesen berücksichtigen die theoretischen Erkenntnisse der Kapitel 2 und 3 sowie das Infrastrukturkonzept interner Arbeitsmärkte.

\subsection{Hypothesenformulierung}

Hypothesen erfüllen hier eine Brückenfunktion in der Überleitung vom theoretischen zum empirischen Teil dieser Untersuchung. Sie dienen der Operationalisierung und Konkretisierung des Erkenntnisinteresses. Bei jeder der hier vorgestellten Hypothesen ist ein Hinweis beigefügt, der AufschluB bietet über die Art des Datenmaterials, mit dessen Hilfe die Hypothesen überprüft werden sollen.

Das zur Verfügung stehende Datenmaterial umfaßt qualitatives und quantitatives Datenmaterial. Das qualitative Datenmaterial erstreckt sich auf Informationen, die in Form von Interviews erhoben wurden. Sie ergänzen das quantitative Datenmaterial. Die Überprüfung der Hypothesen beruht nahezu ausschließlich auf quantitativem Datenmaterial. Eingegangen wird bei dieser Gelegenheit auch auf die Informationen, die zur Überprïfung der Hypothesen dienen bzw. auf die theoretisch abgeleiteten Grundlagen. Auch die Erhebungsquelle wird kurz erwähnt. Weitergehende Informationen, die die Untersuchungsmethodologie betreffen, werden ebenfalls Gegenstand des folgenden Kapitels sein. 
Hypothese 1: Bei der Organisationsform der professionellen Bürokratie ist die intraorganisationale Mobilität (gemessen an der Zahl der Beförderungen) kleiner als bei der Maschinenbürokratie.

Aufgrund der Geschlossenheit intemer Arbeitsmärkte ergibt sich ein nachhaltiger EinfluB des Allokationsgeschehens innerhalb der Organisation (Doeringer/Piore, 1971), da Einflüsse von außen weitgehend ausgeschaltet sind. Maschinenbürokratien repräsentieren diese Geschlossenheit. Akzentuiert wird bei dieser Organisationskonfiguration die Allokationssteuerung von 'innen ' heraus (Sorensen, 1983). Demgegenüber zeigen professionelle Bürokratien mehr 'Marktorientierung' hinsichtlich der Rekrutierung von Personal. Ein mehr interaktives Verhalten mit der 'Außenwelt' bedingt eine Offenheit, die es ermöglicht, auf Impulse von 'außen' einzugehen. Hinsichtlich der intraorganisationalen Mobilität ergeben sich bei Maschinenbürokratien Mobilitätsketten, die über die 'ports of entry' zugänglich sind, jedoch nicht für 'Seiteneinsteiger' auf einem bestimmten Hierarchieniveau. Professionelle Bürokratien stehen 'Seiteneinsteigern' prinzipiell offen. Für die ebenfalls in dieser Organisationkonfiguration vorhandenen Mobilitätsketten bedeutet dies, daß die Funktionskontinuität durch den Zugang auf unterschiedlichen Hierarchieniveaus unterbrochen ist. Die 'zentralen' 'ports of entry' fehlen hier. Aufgrund dieser strukturellen Unterschiede ist die Zahl der Beförderungen in Maschinenbürokratien größer als die in professionellen Bürokratien (Tab. 3).

Die Überprüfung dieser Hypothese erfolgt an Hand von Informationen, die organisationsinterne Beförderungen betreffen. Untersucht wird an Hand des Datenmaterials die Frage: wieviel Fälle intraorganisationaler Mobilität kommen bei der professionellen Bürokratie bzw. bei der Maschinenbürokratie vor (Soeters/Schwan, 1990) und welche Verteilung über Hierarchiestufen läßt sich feststellen? Theoretische Ausgangsbasis für diese Hypothese ist das Infrastrukturkonzept der Beschäftigungskennzeichen. Dieses befindet sich in Kapitel 3, Tabelle 3. Angesprochen werden die Beschäftigungskennzeichen der Mobilitätsketten, Mobilitätsrichtung und Mobilitätsstandards.

Hypothese 2: Während für die Maschinenbürokratie die 'ports of entry' des internen Arbeitsmarktes sich auf unterem Hierarchieniveau befinden und die Möglichkeit des 'Seiteneinstiegs' auf höheren Hierarchieebenen kaum ermöglichen, bestehen bei der professionellen Bürokratie Rekrutierungskanäle auf verschiedenen Hierarchieniveaus.

Neben den in der Erläuterung zu Hypothese 1 zum Ausdruck kommenden strukturellen Unterschieden zwischen den beiden Organisationskonfigurationen bilden Arbeitnehmerqualifikation einen Schüssel für die Unterschiedlichkeit des Zugangs zu der jeweiligen Organisationskonfiguration. Dieser hängt u. a. ab von der betriebspezifischen Weiterbildung und den damit verbundenen Beförderungen bei Maschinenbürokratien. Bei dieser Organisationskonfiguration gilt es, für eintretende Arbeitnehmer ein Lemprogramm zu organisieren, das einen sukzessiven Aufbau von betriebsspezifischer Qualifikation ermöglicht. Bei professionellen Büroksatien (Wholey, 1985; Bills, 1987; Gunz, 1988) steht dagegen 'standardisierte (vielfach exiem erworbene) Qualifikation' im Vordergrund, die sich in Form von allgemein anerkannten Zertifikaten nachweisen läßt. Dies setzt tenden- 
ziell weniger Aktivitäten voraus, den Lemprozeß organisationsintern zu organisieren. Der Markt stellt Personal mit den erwünschten Qualifkationsstandards zur Vertügung.

Die Überprïfung der Hypothese berücksichtigt die Anzahl der internen und extemen Reknutierungen (Goldner/Ritti, 1967) sowie das jeweilige hierarchische Funktionsniveau für die verschiedenen Organisationskonfigurationen vergleichend betrachtet. Theoretische Ausgangsbasis ist die Theorie des internen Arbeitsmarktes (Kapitel 2; das Infrastrukturkonzept: Kapitel 3, Tabelle 3). Vom Infrastrukturkonzept werden hier angesprochen die organisatorische Zugangsmöglichkeit, die 'ports of entry' und die Mobilitätsstandards.

\subsubsection{Hypothese mit Bezug auf Gehaltszuwachsraten}

Hypothese 3: In Maschinenbürokratien sind die Gehaltszuwachsraten als Indikator für Beförderung und betriebliche Bindung höher als in den professionellen Bürokratien entwickelt.

Das theoretische Fundament dieser Hypothese basiert auf der Perspektive der Arbeitnehmerbindung in intemen Arbeitsmärkten, die als finanzielle Anreizstruktur wahmehmbar ist. So ist bei Maschinenbürokratien ein stärkerer Bindungseffekt zwischen Arbeitnehmer und Organisation zu konstatieren als vergleichsweise bei professionellen Bürokratien. Konkret wird erwartet, daß die Gehaltszuwachssteigerung bei Maschinenbürokratien stärker zunimmt als bei professionellen Bürokratien. Zur Überprïfung der Hypothese ist ein Vergleich der Zuwachsraten der monatlichen Bruttogehälter im Vierjahreszeitraum zwischen professioneller und Maschinenbürokratie vorgesehen (Bills, 1987; DiTomaso, 1989). Das theoretische Fundament dieser Hypothese basiert auf einen Teil der Theorie des internen Arbeitsmarktes (Kapitel 2 und 3). Ausgegangen wird von einer Koppelung von Einkommen und Arbeitsplätzen (Ross, 1981; Granovetter, 1988; Ker, 1954). Aufgrund der differenzierteren Funktionsstrukrur in Maschinenbürokratien gegenüber professionellen Bürokratien dürften die Gehaltszuwächse bei der erst genannten Organisationskonfiguration höher ausfallen (Rosenbaum, 1979b). Angesprochen werden hier aus dem Infrastrukturkonzept Design Parameter für Mobilitätsketten insbesondere die Jobbindung sowie die Mobilitätsketten.

\subsubsection{Hypothesen mit Bezug auf die Beschäftigungsdauer}

Hypothese 4: Die Beschäftigungsdauer bzw. Organisationszugehörigkeit von Arbeitnehmern nimmt in Maschinenbürokratien mit höherem Funktionsniveau mehr zu als in professionellen Bürokratien.

Hypothese 5: Die Intensität der Funktionswechsel bei beiden Organisationskonfigurationen ist zu Beginn der Organisationszugehörigkeit von Arbeitnehmern größer als in späteren Phasen.

Die Überprüfung beider Hypothesen soll zum einen die Verbleibdauer von Arbeitnehmem auf bestimmten Funktionen analysieren. Damit wird auf die Bindung von Arbeitnehmem auf internen Arbeitsmärkten abgestellt. Die lange Verbleibdauer in einer Funktion spricht dafür, daß schnelle, karrierebezogene Funktionswechsel nicht beabsichtigt sind. Durch die 
Pfadabhängigkeitsthese von Rosenbaum (1984) wird aufgezeigt, daß Karrierechancen zu Beginn der Organisationszugehörigkeit in Form von Funktionswechsel bessere Fortkommenschancen eröffnen. Funktionswechsel von Arbeitnehmern vollziehen sich in der Anfangsphase der Beschäftigungszugehörigkeit zur jeweiligen Organisation. Unterschiedliche Bedingungen können hiermit verbunden sein. Dabei geht es um die Suche des Arbeitnehmers nach Stabilität innerhalb seiner Funktion im Rahmen des betrieblichen Sozialisationsprozesses. Auch Umbesetzungen aufgrund von Fehlbesetzungen sowie 'Springerfunktionen' fallen unter diese Hypothesen (Bills, 1987), Außerdem läßt sich 'job rotation' dazu rechnen mit dem Ziel, 'Generalisten' zu fördern.

Ausgangsbasis ist zum einen die Langfristigkeit der Beschäftigung auf internen Arbeitsmärkten als ein für inteme Arbeitsmärkte konstituierendes Merkmal. Zum anderen wird Bezug genommen auf das Modell von Rosenbaum (1979b) in oben genanntem Sinne als einer der Komponenten der Erklärung organisationsintemer Allokation von Arbeit (Kapitel 3). Vom Infrastrukturkonzept (Tabelle 3) werden hier ansprochen die Elemente 'qualifikationserhöhende Funktion' sowie 'Häufigkeit der Funktionsänderung'.

\subsubsection{Hypothesen zur Besetzung offener Stellen}

Hypothese 6: Die Besetzung offener Stellen erfolgt bei den Maschinenbürokratien in der Regel intern, bei den professionellen Bürokratien meistens extern.

Die Überprüfung dieser Hypothese geht von der Vorstellung aus, daß die interne Arbeitsmarktdynamik bei Maschinenbürokratien größer ist als die in professionellen Bürokratien (Gerpott 1988; Berthel 1987; Bills 1987; Mintzberg, 1979). Die Besetzung offener Stellen erfolgt daher bei Maschinenbürokratien aus dem bestehenden Personalbestand. Im Gegensatz dazu wird bei professionellen Bürokratien mehr der externe Arbeitsmarkt bemüht bei der Besetzung offener Stellen. Geprüft wird, in welchen quantitativen Relationen interne und exteme Rekrutierungen vorgenommen werden und wie sich diese auf die Organisationskonfigurationen verteilen. Die theoretische Basis hierfür ist das Modell von DiPrete (Kapitel 3) sowie die äußere Organisationsgrenze als 'Schnittstelle' für die Interaktionen zwischen in- und extemer Allokation von Arbeit (Soerensen, 1983) (Kapitel $2+3$ ). Das Infrastrukturkonzept (Tabelle 3) der Beschäftigungskennzeichen spricht mit dieser Hypothese die folgenden Elemente an: die Offenheit bzw. Geschlossenheit von internen Arbeitsmärkten, die berufliche Zugangsmöglichkeit sowie die Überwindung organisatorischer Grenzen.

Hypothese 7: Befindet sich die offene Stelle auf einer Funktionskette, so erfolgt bei Maschinenbürokratien die Stellenbesetzung in der Regel intern und dann aus derselben Abteilung und Funktionskette.

Ausgangspunkt ist die Vorstellung, daß in Maschinenbürokratien eine Infrastruktur in Form von Funktionsketten besteht (Baron u. a., 1986; Diprete, 1987a). Beim Entstehen einer offenen Stelle soll diese möglichst schnell 'pragmatisch' besetzt werden. Potentielle Kandidaten befinden sich in der nächsten Umgebung, d. h. innerhalb derselben Abteilung bzw. auf derselben Funktionskette. Aus Transaktionskostengründen kommen potentielle Kandidaten aus der nächsten Umgebung eher für die Stellenbesetzung in Frage als 'ent- 
ferntere' Kandidaten, da bei diesen zusätzliche Informationen, d. h. mehr Aufwand erforderlich wird, was wiederum nit Kosten verbunden ist. Die unterschiedliche Präferenz bei den Organisationskonfigurationen läßt sich mil den praktizienten Qualifikationsstandards erklären (Tolbert/Zucker, 1983).

Bei Maschinenbürokratien ist die Qualifikation aufgabenbezogen. Daraus entsteht eine Besetzungspräferenz zugunsten von Personen, die mit der Aufgabe einigermaßen vertraut sind und aus der nächsten Umgebung der Vakanz stammen. Im Gegensatz dazu ist die Besetzungspräferenz bei den professionellen Bürokratien auf extern erworbene Qualifikationsstandards konzentriert, was eine 'weiträumigere' Suchaktivität beinhaltet. Die empirische Prüfung vollzieht sich über Allokationspräferenzen innerhalb von organisatorischen Allokationsgrenzen, konzipien in Abbildung 10, Kapitel 3. Das theoretische Fundament dieser Hypothese liegt in Williamsons Transaktionskostenansatz (Kapitel 3) und im Modell der offenen Stellenbesetzung von DiPrete (Kapitel 3). Im Rahmen des Infrastrukturkonzeptes werden hier angesprochen die Überwindung organisatorischer Grenzen, die Zahl der Jobs auf der Jobleiter sowie die Mobilitätsstandards.

Hypothese 8: In professionellen Bürokratien mit einer hohen Personalbewegung geht die Besetzungspräferenz offener Stellen bei ausschließlich interner Besetzung in Richtung der Besetzung mit Personen aus einer anderen Abteilung und derselben Funktionskette.

Bei professionellen Bürokratien wird die Suche nach Personal mit dem Ziel verfolgt, möglichst einen vergleichbaren Qualifikationsgrad bei potentiellen Kandidaten aus einer vergleichbaren Berufsgruppe zur Verfügung zu haben. Die Besetzungspräferenz (Bills, 1987; Wholey, 1985; Gerpott, 1988) geht daher in Richtung der Rekrutierung von Kandidaten aus anderen Abteilungen mit einem vergleichbaren Funktionsniveau auf der Funktionskette. Die standardisierten Qualifikationsniveaus von Arbeitnehmem in professionellen Bürokratien haben einen stärkeren Einfluß auf die Besetzungspräferenz als die Funktionsnuancen bei Funktionsleitem in Maschinenbürokratien. Auch hier soll die empirische Prüfung über Allokationspräferenzen innerhalb von organisatorischen Allokationsgrenzen erfolgen, konzipiert in Abbildung 10, Kapitel 3. Das theoretische Fundament dieser Hypothese liegt in Williamsons Transaktionskostenansatz (Kapitel 3) und im Modell der offenen Stellenbesetzung von DiPrete (Kapitel 3). Auch hier wird im Rahmen des Infrastrukturkonzepts (Tab. 3) intemer Arbeitsmärkte die Überwindung organisatorischer Grenzen angesprochen.

Nachdem nun alle Hypothesen vorgestellt wurden, soll im folgenden Kapitel Einblick in die Methodologie der Untersuchung gegeben werden. 


\section{$5 \quad$ Methoden der empirischen Untersuchung}

In diesem Kapitel steht die Untersuchungsmethodologie im Mittelpunkt. Eingegangen wird auf die Auswahl der untersuchten Organisationen. Außerdem wird aufgezeigt, welche Untersuchungsinstrumente und -verfahren hier Anwendung finden.

Schließlich bedingte das Erkenntnisinteresse die Kombination unterschiedlicher Methoden (Patzelt, 1985; Yin, 1985), die in diesem Kapitel kurz vorgestellt werden. Ziel war es, eine explorative Untersuchung vorzunehmen, wobei eine Vielzahl organisationsspezifischer Daten gewonnen wurde. Die Zahl der in die Untersuchung eingegangen Organisationen wurde auf vier beschränkt zugunsten einer intensiveren Analyse der organisationsinternen Dynamik, aber auch des Vergleichs der Organisationen untereinander.

\subsection{Kriterien der Auswahl der untersuchten Organisationen}

Die Auswahl der untersuchten Organisationen erfolgt nach Kriterien des internen Arbeitsmarktes so wie sie von Doeringer/Piore (1971) in Kapitel 2 analytisch angesprochen wurden. Außerdem werden die Kriterien der Definition des internen Arbeitsmarktes nach Lutz (1987) einbezogen. Mehr oder weniger als Kontrast wurde jene Organisationsform gewählt, bei der überwiegend professionelle Arbeitnehmer anzutreffen sind. In der Literatur werden diese unterschiedlichen Arbeitsmarktformen gegenübergestellt. Empirische Evidenzen wurden in den voraufgehenden Kapiteln angedeutet (Biehler/Brandes, 1981; Soeters/Schwan, 1990). Jeder Autor hat seine eigene Akzentsetzung. Dennoch zeigt sich wie angedeutet - ein hohes $\mathrm{Ma} B$ an Übereinstimmung bei den Organisationstypen zum Beispiel zwischen Williamson und Mintzberg.

Die Mintzberg Organisationstypologie bietet neben der Einteilung in Organisationskonfigurationen auch ein organisationsintemes Analyseinstrumentarium, das die empirische Operationalisierung komplexer Organisationen erleichtert und vor allem vergleichbar macht (Soeters/Schwan, 1990). Williamson ermöglicht mehr die ökonomische Einschätzung von Organisationsstrukturen im Rahmen der 'governance structure' und eine Einschätzung von deren Vorteilhaftigkeit gegenüber alternativen Transaktionskosten.

Um den forschungsmäßigen Zugang zu den jeweiligen Organisationskonfigurationen zu finden, soll in erster Linie auf das von Mintzberg entwickelte Instrumentarium zurückgegriffen werden. Die Selektion von Organisationen in der Realität erfolgt nach den Kriterien der idealtypischen theorieorientierten Beschreibung von Maschinenbürokratie und professioneller Bürokratie. Als Organisationen, die dem Typ der Maschinenbürokratie weitgehend entsprechen, wurden zwei Organisationen ausgewählt: zum einen ein Produktionsuntemehmen und zum anderen ein Sozialversicherungsuntemehmen. Als Organisatio- 
nen, die weitgehend dem Typ der professionellen Bürokratie entsprechen, wurde ein Krankenhaus und eine Wirtschaftprüfungsgesellschaft ausgewählt.

Abb. 11: Die in die empirische Untersuchung eingegangenen Organisationen

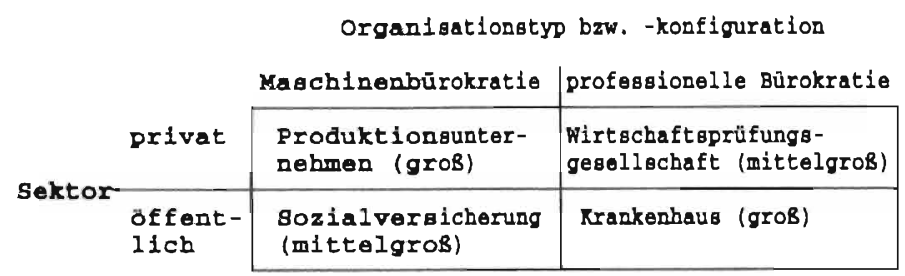

Die Auswahl der Organisationen zum Zwecke der empirischen Untersuchung wurde außer von den bereits genannten von weiteren Differenzierungskriterien bestimmt. Dazu gehört die Aufteilung in große und mittelgroße Organisationen, die sich wie folgt umschreiben lassen: in dieser Untersuchung gelten als große Organisationen jene, die 880 bzw. 820 Mitarbeiter, als mittelgroße Organisationen jene, die 165 bzw. 365 Mitarbeiter umfassen.

Bei den untersuchten Organisationen entsprechen Krankenhaus und Produktionsunternehmen der Kategorie 'groß', während Sozialversicherung und Wirtschaftsprüfungsgesellschaft der Kategorie 'mittelgroB' entsprechen. Als weiteres Differenzienungkriterium findet die Einteilung in öffentliche bzw. private Organisationen hier Anwendung. Bei den untersuchten Organisationen sind Krankenhaus und Sozialversicherung öffentlich, während Produktionsunternehmen und Wirtschaftsprüfungsgesellschaft als private Einrichtungen zu betrachten sind.

Mintzberg bietet u. a. ein organisationstheoretisches und analytisches Instrumentarium zur Beschreibung der intemen Struktur von Organisationen. Im folgenden soll kurz auf die 'Organisationsteile' als grobe Differenzienung der Organisation eingegangen werden, die die Anlage der empirischen Untersuchung beeinfluBten. In den Vorkapiteln war von 'parts' die Rede.

Es handelt sich um eine von der jeweiligen Organisationskonfiguration unabhängige Aufteilung in "parts", also Organisationsteile. Diese sind in Abhängigkeit von der jeweiligen Organisationskonfiguration unterschiedlich ausgeprägt. Die Organisation besteht nach Mintzberg (1979) aus folgenden Organisationsteilen (parts): 
Abb. 12: Idealtypische Organisation mit Andeutung der Organisationsteile (parts) nach Mintzberg
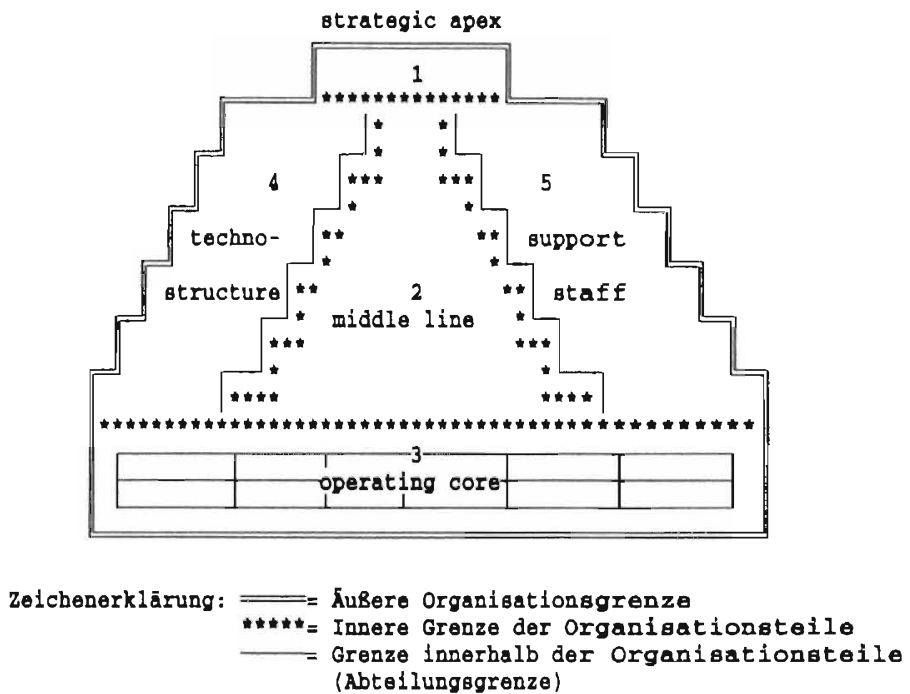

Quelle: nach Mintzberg, 1979

Teil 1 umfaßt die Organisationsleitung (strategic apex), wo wichtige, den Bestand der Unternehmung betreffende Fragen entschieden werden. Teil 2 umfaßt die Hierarchie unterhalb der Organisationsleitung bis hin zur arbeitsausführenden Ebene, auch "middle line" genannt. Die eigentliche arbeitsausführende Ebene wird in der Terminologie von Mintzberg als "operating core" bezeichnet. Als Teil 4 "technostructure" der Organisation werden jene Abteilungen betrachtet, die eine analytische (intelligente) Dienstleistung (Befugtheit) für die Organisationsteile 1-3 bieten. Sie tragen bei zur Standardisierung der Arbeit, kontrollieren Aktivitäten innerhalb der Organisation und haben oft den Charakter einer Stabsstelle. Hierbei ist beispielsweise an die Personalleitung und die Finanzabteilung gedacht. Kennzeichnend für den Teil 4 der Organisation ist weiter, daB er meistens nicht in den eigentlichen 'Organisationskorpus' integriert ist. Dies trifft auch für Teil 5 der Organisation zu, der als "support staff" bezeichnet wird. Hiermit sind jene Hilfsfunktionen gemeint, die die zentrale Aufgabenstellung der Organisation unterstuitzen, ohne jedoch einen direkten Einfluß darauf zu haben im Gegensatz zur "technostructure". Als Beispiel läßt sich die Poststelle, die Kantine aber auch die juristische oder die public relation Abteilung anfuihren.

Unter anderem wird bei den im empirischen Teil aufgeführten Detailanalysen auf die Differenzierung in "parts" zurückgegriffen.

Aus der Methodenvielfalt empirischer Sozialforschung (Yin, 1985) wurde wie oben angedeutet eine Methodenkombination gewählt, die sowohl eine quantitative als auch eine 
qualitative Untersuchung ermöglicht. In den folgenden Abschnitten wird näher darauf eingegangen.

\subsection{Die qualitative und quantitative Untersuchung}

\subsubsection{Die qualitative Untersuchung}

Die qualitative empirische Untersuchung bestand aus vier strukturierten Interviews per Organisation. Sie dienten dem Zweck, qualitative Informationen über die jeweilige Beschäftigungssituation in den einzelnen Organisationen zu bekommen. Demgegenüber steht eine quantitative empirische Erhebung, deren Gegenstand und Hintergründe im folgenden Abschnitt erläutert werden sollen.

Für die Interviews wurde ein Gesprächsleitfaden entwickelt, aus dem später das standardisiente Interview entstand (Atteslander, 1984; König, 1979). Die Interviews enthielten zahlreiche Themen, die für den jeweiligen Gesprächspartner qua Aufgabengebiet von Relevanz waren. Die in großem Maße vorstrukturierten Fragen wurden im Interview so angeordnet, daß die Gesprächsthemen ohne Kontextbrüche aufeinander aufbauten. Dort, wo dies nicht möglich war, wurde der Gesprächspartner durch eine Überleitung auf einen neuen Gesprächsgegenstand eingestimmt. Die Vorstrukturierung der Fragen erwies sich als nützlich sowohl aus zeitökonomischen als auch aus Gründen der Vergleichbarkeit. Aufbau und Strukturierung des Interviews ließen Freiraum, um in bestimmten Fällen vom Gesprächsleitfaden abzuweichen zur qualitativen Ergänzung bzw. Vertiefung der erhaltenen Informationen.

Die Antworten waren größtenteils als Struktur bzw. Auswahlmöglichkeit vorgegeben, soweit es sich um entsprechende Fragen handelte. Bei einem kleinen Teil der Fragen war eine offene Antwort vorgesehen. In bestimmten Fällen wurde eine fünfstufige Likert-Skala als Antwortmöglichkeit vorgegeben.

Drei strukturierte Interviews per Organisation plus einem Fragebogen zur vorbereitenden quantitativen Informationsgewinnung waren mit dem Ziel aufgebaut, auf Grund der unterschiedlichen Kompetenzniveaus der Interviewpartner und deren spezifischer Sicht Informationen zu bekommen. Die Konstruktion der Interviews (Schnell, Hill, Esser, 1988) war so angelegt, daß bestimmte Gesprächsgegenstände sich in leicht modifizierter Form in anderen Interviews zurückfinden ließen. Dies diente der besseren Vergleichbarkeit der Aussagen der Gesprächspartner, aber auch der Gewährleistung der Validität und Reliabilität der Aussagen. In mehreren Fällen standen zwei Interviewpartner für ein Interview zur Verfügung. In diesen Fällen erfolgte die Antwort auf die Fragen koordiniert, d. h. die Interviewten stimmten die Antwort untereinander ab, soweit dazu Anlaß bestand. Die Interviews wurden 1984 durchgeführt.

Interview 1 wurde als sogenanntes 'Eingangsinterview' benutzt. Im Abschnitt "Methode der Abnahme der Interviews" wird die Bedeutung dieses Interviews näher erläutert. Interview I war auf den Leiter der Personalabteilung zugeschnitten. Inhaltlich wurden die Grundsätze der Personalpolitik, Struktur der Organisation, Struktur der Arbeitsplätze und 
Personalprobleme, interne Mobilität und das Verhältnis zum externen Arbeitsmarkt angesprochen.

Interview 2 hatte strategische Perspektiven der Organisation zum Gegenstand. Gesprächspartner war in diesem Fall ein Mitglied der Geschäftsleitung. Die Fragen richteten sich auf die strategischen Perspektiven der Organisationsentwicklung, die Rolle der Personalpolitik als Teil der Untemehmenspolitik und auf Themen wie strategische Informations- und Entscheidungsfindung mit Bezug auf die betriebliche Beschäftigungssituation.

Interview 3 umfaßte Personalentwicklung, Laufbahn-bzw. Karriereplanung sowie Struktur von Funktionen in Form von Funktionsketten und die intraorganisationale Mobilität von Arbeitnehmern. Dieses Interview richtete sich an Personen der Personalabteilung, die mit Personalentwicklung bzw. -begleitung verantwortlich beschäftigt waren.

Ein weiterer Fragebogen galt der Sammlung statistischer Informationen über die Organisation und zugleich der Vorbereitung der quantitativen Erhebung. Im einzeinen wurden demographische sowie personalbezogene statistische Informationen abgefragt. Dabei handelt es sich um auf Organisationsniveau aggregiertes Datenmaterial, also keine auf den individuellen Arbeitnehmer bezogenen, personenbezogenen Daten. Adressat war eine Person der Personalabteilung, die mit der Aufgabe der Personaldatenaufbereitung bzw. mit dem Personalinformationssystem befaßi war.

Die Ergebnisse der qualitativen Untersuchung werden zum überwiegenden Teil in Kapitel sechs verarbeitet in Form der Beschreibung der untersuchten Organisationen.

\subsubsection{Kontaktanbahnung zu den Organisationen}

Die untersuchten Organisationen wurden angeschrieben und um Mitwirkung bei der Untersuchung gebeten. Angeschrieben wurde eine Person, die im Bereich der Geschäftsleitung anzusiedeln ist. Kurze Zeit nach Versenden der Bitte um Mitwirkung wurde telefonisch Kontakt aufgenommen mit dem Adressat zwecks Besprechung der Untersuchung und Erläuterung des Vorhabens. Zur Sozialversicherung bestanden gute Kontakte, zumal die Organisation im Rahmen eines interaktiven Lehrprozesses an der Universität mitwirkte und auch für Untersuchungen anderen Inhalts (Soeters/Schwan, 1990; Kunst et al., 1989; Romme et al., 1989; Schreuder et al., 1988) zugänglich war.

Gemeinsam (mit der Geschäftsleitung) wurde überlegt, aus welchen Quellen welche Informationen zu gewinnen sind. Daruberhinaus wurde geklärt, auf welche Weise gewünschte Informationen zur Verfügung stehen und welche Schritte von Seiten der Organisation ergriffen werden, um nicht direkt verfügbare Informationen aufzubereiten, so daß sie für den Zweck der Untersuchung erhältlich waren. Dies erschien insoweit notwendig, da die Informationen nicht "abrufbar" zur Verfügung standen. Zum Teil mußten erhebliche Anstrengungen unternommen werden, um an bestimmte Informationen zu kommen, da diese von Organisation zu Organisation variierend dokumentiert wurden.

Die drei Interviews waren so aufgebaut, daß die Rangfolge zugleich eine Reihenfolge darstellte. Ziel war es, die Informationsgewinnung schrittweise zu ermöglichen. Die 
Abnahme der Interviews erfolgte durch den Autor und einer Begleitperson, deren Funktion darin bestand, Mißverständnisse in der Kommunikation, soweit sie entstanden, auszuräumen und die Antworten zu protokollieren bzw. die Antworten anzukreuzen. Neben dem psychologischen Beistand sollte auf diese Weise die Wahmehmung einen höheren Grad an Reliabilität erhalten.

\subsubsection{Methode der Abnahme der Interviews}

Die Abnahme der Interviews sowie die Vorgespräche wurden in niederländischer Sprache geführt. Vor dem erstmaligen Einsatz der Interviews wurden mehrere Pretests vorgenommen, die Änderungen bzw. Verbesserungen der Fragen bzw. Fragenfolge nach sich zogen.

So diente das Eingangsinterview der Orientienung und Planung der darauf folgenden Untersuchungsschritte. Zunächst ging es darum, eine Vorstellung von der jeweiligen Organisation zu gewinnen. Dann erfolgte eine vorläufige Einteilung der Organisation nach den analytischen Kriterien von Mintzberg. Dabei ging es um die Einteilung der Parts und die Vorbereitung der quantitativen Erhebung durch eine in Relation zur Größe der Parts vorzunehmende Stichprobe aus der Gesamtzahl der im jeweiligen Part anzutreffenden Arbeitnehmer.

Die anderen Interviews wurden in der oben erwähnten Reihenfolge abgenommen. Die Dauer eines Interviews lag zwischen ein und zwei Stunden. Fragebogen 4 bezog sich auf quantitatives Zahlenmaterial zur Beschäftigungssituation bzw. zur Personalpolitik. Dieses Interview war durch zum Teil erhebliche Aktivitäien von Seiten des Interviewpartners gekennzeichnet, die getragten Informationen herbeizuschaffen. Das Kommunikationsklima war aufgeschlossen und von der Bereitschaft charakterisiert, wohlwollend die gewünschten Informationen zur Verfügung zu stellen. Aufgrund des Umfangs der Fragebögen soll darauf verzichtet werden, diese im Arhang aufzunehmen.

\subsubsection{Die quantitative empirische Untersuchung}

Die quantitative empirische Untersuchung beinhaltete pro Organisation eine Analyse der Infrastruktur interner Arbeitsmärkte und eine offene Stellenanalyse.

\subsubsection{Die Infrustrukturanalyse intemer Arbeitsmärkte}

Gegenstand der Infrastrukturanalyse war die Analyse von Beschäftigungskennzeichen innerhalb von Organisationen. Der Analysezeitraum umfaßte fünf Jahre. Erfaßt wurde die Mobilität, die sich von 1984 bis 1988 vollzog. Mobilität wird umfassend verstanden. Gemeint sind Beförderungen, Veränderungen von Funktionen, die sowohl horizontale als auch vertikale Mobilitätsrichtungen einschließen.

Der Infrastrukturanalyse liegt das Auswahlverfahren der 'Zufailsstichprobe' (Schnell, Hill, Esser, 1988) zugrunde. Die Analyse basiert auf einer geschichteten Stichprobe von durchschnittlich 75 Beschäftigten pro Organisation, die nach den fünf Parts unterverteilt waren, so daß durchschnittlich pro Part 15 Arbeitnehmer nach dem Zufallsprinzip ausgewählt wurden. $\mathrm{Da}$ die Anzahl der Arbeitnehmer bzw. die Stellenbesetzungen in den 
verschiedenen Parts unterschiedlich ausgeprägt waren, wurde dem Umfang der Stellenbesetzung in der Stichprobe Rechnung getragen. Als Kriterium dafür war von Bedeutung, daß möglichst alle Parts, soweit es das Beschäftigungsvolumen zuließ, voll in die Stichprobe eingingen. Die Infrastrukturanalyse konzentrierte sich auf drei Teilanalysen, die im folgenden kurz vorgestellt werden.

Im Mittelpunkt steht daher eine Beförderungsanalyse, bei der sämtliche Beförderungen in vertikaler und horizontaler Richtung im Rahmen der Stichprobe innerhalb des Untersuchungszeitraums von fünf Jahren erfaßt wurden. Unter horizontaler Beförderung ist der Wechsel auf ein vergleichbares Funktionsniveau zu verstehen, jedoch mit einer Zunahme an Verantwortlichkeit bzw. Kontrollspanne.

Als eine andere Art der Erfassung eines strukturellen Unterschiedes zwischen Maschinenbürokratien und professionellen Bürokratien gilt die Gehaltszuwachsentwicklungsanalyse. Die Gehaltszuwachsentwicklung basiert auf den durchschnitllichen Zuwachs des Bruttomonatsgehaltes von Jahr zu Jahr innerhalb des untersuchten Zeitraumes.

Neben diesen Analysen wurde außerdem eine Analyse der Beschäftigungszeiten (-dauer) durchgeführt auf der Grundlage der 'Mobilitätsstichprobe'. Der erfaßte Zeitraum, als langfristige Analyse angelegt, geht deutlich über den der Beförderungsanalyse zugrunde liegenden Zeitraum von fünf Jahren hinaus. Bei dieser Untersuchung wurde der Häufigkeit des Funktionswechsels innerhalb der Organisation besonders Rechnung getragen. Im empirischen Teil der Arbeit wird auf die Einzelheiten der Methode ausfuihrlicher eingegangen.

Für die Erhebung war es von Interesse, ausgehend von der realen Verteílung der Beschäftigten, diese in etwa durch die Gewichtung der Stichprobe zu berücksichtigen. Im einzelnen ergibt sich für die jeweilige Organisation folgende Verteilung, wobei hier die Stellenbesetzung innerhalb der Parts mit der Stichprobe verglichen wird. 
Tab. 4: Stichprobe und Stellenbesetzung zum Erhebungsjahr 1989

\begin{tabular}{|c|c|c|c|c|c|c|c|c|c|c|c|}
\hline \multirow[b]{3}{*}{ Parto } & \multicolumn{11}{|c|}{ Organibation } \\
\hline & \multicolumn{3}{|c|}{$\pi$} & \multicolumn{2}{|c|}{ W } & \multicolumn{2}{|c|}{$s$} & \multicolumn{2}{|l|}{ P } & \multicolumn{2}{|c|}{ Total } \\
\hline & G & $\mathbf{B}$ & $\mathbf{T}$ & B & T & B & $T$ & B & T & 8 & $\mathrm{~T}$ \\
\hline $\begin{array}{l}\text { Strategic } \\
\text { apex }\end{array}$ & 15 & 8 & 3 & 10 & 10 & 3 & 2 & 4 & 4 & 25 & 19 \\
\hline $\begin{array}{l}\text { Middle } \\
\text { IIne }\end{array}$ & 15 & 62 & 14 & 30 & 6 & 28 & 16 & 15 & 15 & 135 & 51 \\
\hline $\begin{array}{l}\text { Operating } \\
\text { core }\end{array}$ & 15 & 432 & 20 & 100 & 46 & 287 & 43 & 423 & 29 & 1248 & 132 \\
\hline $\begin{array}{l}\text { Techno- } \\
\text { structure }\end{array}$ & 15 & 52 & 15 & 6 & 2 & 2 & 2 & 42 & 15 & 102 & 34 \\
\hline $\begin{array}{l}\text { Support } \\
\text { staff }\end{array}$ & 15 & 326 & 23 & 19 & 13 & 45 & 13 & 336 & 15 & 720 & 70 \\
\hline Total & 75 & 880 & 75 & 165 & 77 & 365 & 76 & 820 & 78 & 2230 & 306 \\
\hline
\end{tabular}

Total: Gesamtzahl der Beschäftigten: 2230

Gesamtzahl der Beschäftigten in der Stichprobe: 306

Zeichenerklärung: $\mathrm{K}=\mathrm{Krankenhaus;} \mathrm{W}=$ Wirtschaftsprüfungsunternehmen; S=Sozialversicherung; $\mathrm{P}=$ Produktionsuntemehmen; $\mathrm{G}=$ Geplante Stichprobe in jeder Organisation; B=Besetzung der Stellen mit Anzahl Mitarbeiter; T=Tatsächliche Stichprobe

Die Abweichung der tatsächlichen von der geplanten Stichprobe ist darauf zurückzuführen, daß die jeweiligen Parts unterschiedlich mit Personal besetzt sind. Die tatsächliche Stichprobe trägt der Gewichtung der Zahl der Beschäftigten so weit wie möglich in den Parts Rechnung.

Die Rekonstruktion der organisationsinternen Mobilität erfaßte die zum Zeitpunkt der Erhebung beschäftigten Mitarbeiter im Rahmen der Stichprobe. Die Datensammiung erfolgte auf der Basis von Personalakten bzw. unter Zurhilfenahme des Personalinformationssystems (soweit vorhanden). Dabei wurde den beteiligten Interviewpartnem bzw. Gesprächspartnern die vertrauliche Behandlung der erhaltenen Informationen zugesichert. Der Gewährleistung dieses Anspruchs wurde dadurch Rechnung getragen, daß die für die Erhebung entwickelten Formulare von vomherein auf Anonymität basierten. Bei der anschließenden Datenverarbeitung wurden die Informationen in kodierter Form verarbeitet, so daB keine Rückschlüsse auf Ursprung oder bestimmte Kontexte möglich waren. Dariberhinaus lag die Verarbeitung der empirisch gewonnen Informationen ausschließlich in der Hand des Autors. Um datenschutzrechtlichen Problemen aus dem Weg zu gehen, wurden teilweise unter erheblichem Aufwand von den Personalakten anonymisierte Fotokopien zu Analyse- bzw. Erhebungszwecken gemacht. Die aus Personalinformationssystemen gewonnenen Informationen wurden ebenfalls in anonymisierter Form zur Vertügung gestellt. Die Vorstellung der Variablen erfolgt im Rahmen des empirischen Teils der Untersuchung. 


\subsubsection{Die offene Stellenanalyse}

Ziel der offenen Stellenanalyse innerhalb der Organisation war es, Aufschluß über die interne Stellenbesetzung zu erhalten. Im Vordergrund stand in Ergänzung zur Infrastrukturanalyse das Entstehen und die Besetzung offener Stellen sowie die mögliche inteme Reallokation der Beschäftigten.

Bei der offenen Stellenanalyse handelt es sich um eine Einzelfallanalyse (Schnell, Hill, Esser, 1988) in Form der Vollerhebung. Berücksichtigt wurden alle offenen Stellen, die im Laufe von drei Jahren entstanden sind. Die Erhebung umfaßt den Zeitraum von 1986 bis 1988 .

Da offene Stellen spätestens zum Zeitpunkt ihrer Besetzung nicht mehr für die Unternehmen interessant sind, ergab sich die Schwierigkeit, auf eindeutige Informationsquellen zurïckzugreifen. Diese Schwierigkeit wurde durch die Heranziehung verschiedener Quellen umgangen. Diese bestanden in internen Stellenausschreibungsdokumenten, aber auch in Personalanzeigen, die sich auf den extemen Arbeitsmarkt richteten. Außerdem wurden sämtliche Beförderungen, Umbesetzungen und Organisationsaustritte sowie die Schaffung neuer Arbeitsplätze erfaßt, also Ursachen, die eine Reallokation, d. h. Wiederbesetzung von offenen Stellen nach sich ziehen. Für die vier Organisationen wurden dabei über den Zeitraum von drei Jahren 962 offene Stellen registriert. Im empirischen Teil dieser Untersuchung kommt zum Ausdruck, daß die Zahl der Beschäftigten im Untersuchungszeitraum nicht konstant war.

Tab. 5: Verteilung der offenen Stellen auf die Organisationen zum Erhebungsjahr 1989

\begin{tabular}{|c|c|c|c|c|c|c|c|c|}
\hline Organisation & $\begin{array}{l}\text { Zahl der } \\
\text { Beschätigten }\end{array}$ & $\begin{array}{l}\text { Offen } \\
1986\end{array}$ & $\underset{\%}{e} \mathrm{St}$ & $\begin{array}{l}\text { ellen } \\
1987\end{array}$ & $\%$ & 1988 & $\%$ & Total \\
\hline Rrankenhaus & 880 & 125 & 14 & 128 & 14 & 128 & 14 & 381 \\
\hline $\begin{array}{l}\text { Wirtschafts- } \\
\text { prüfungeges. }\end{array}$ & 165 & 9 & 5 & 19 & 12 & 17 & 10 & 45 \\
\hline $\begin{array}{l}\text { Sozialver- } \\
\text { bicherung }\end{array}$ & 365 & 84 & 23 & 95 & 26 & 78 & 21 & 257 \\
\hline $\begin{array}{l}\text { Produktions- } \\
\text { unternehmen }\end{array}$ & 820 & 80 & 10 & 102 & 12 & 97 & 12 & 279 \\
\hline
\end{tabular}

Zeichenerklärung: \% = in Prozenten

Die Erläuterung der obigen Tabelle sowie die Vorstellung der Variablen sind dem empirischen Teil der Arbeit vorbehalten.

Die in Kapitel 4 vorgestellten Hypothesen sollen hinsichtlich der ihnen zugrunde liegenden Variablen im Kontext der Darstellung der empirischen Resultate operationalisiert werden. Eine ausführliche Definition der Variablen mit der quantitativen Unterbauung erfolgt in den empirischen Kapiteln 7 und 8. 


\subsection{Methodische Verantwortung der Untersuchung}

Die Untersuchung wurde unter Hinzuziehung statistischer Methoden durchgefühn. In diesem Abschnitt soll die methodische Verantwortung begründet werden.

Bei dieser Studie handelt es sich um eine hypothesentestende Untersuchung, die einem sozialwissenschaftlichen Untersuchungsdesign entspricht. Damit wird der Untersuchungscharakter als beschreibend/prüfend angelegt. Hinzu kommt der explorative Charakter der Untersuchung. Dieser konkretisiert sich in der Zahl der in die Untersuchung einbezogenen vier Organisationen. Zugunsten von Regressions- und Diskriminantenanalysen wurde auf Logit/Probitanalysen verzichtet.

Angelegt wurden zwei unterschiedliche Datenbestände. Einer betrifft die Infrastrukturanalyse interner Arbeitsmärkte, der andere die Analyse der Besetzung offener Stellen. Alle statistischen Analysen wurden in der Software SPSS erstellt. Benutzt wurden die SPSSVersionen 2.1 und 3.1 (SPSS-User's Guide 1986; 1990).

Der Einsatz der statistischen Methoden erfolgte in abgestufter Reihenfolge. Zunächst wurde die Ausprägung einzelner Variablen in Form deskriptiver Statistik präsentiert. In einem weiteren Schritt wurden Verfahren vergleichender beschreibender Statistik verwendet. Dazu lassen sich die SPSS-spezifischen Verfahren wie breakdown, (con-) descriptive (Varianzanalysen) sowie crosstabs, pearson correlation sowie partial correlation anfühuren. Die Varianzanalysen umfaßten die Variablen, die in der jeweiligen Analyse als zu erklärende und erklärende Variablen benutzt wurden. Die durchgeführten Signifikanztests beruhen auf der Varianzhomogenitäts- und Normalverteilungsannahme. Die oben erwähnten statistischen Analysen vermittelten Einsichten in die Struktur des Datenmaterials. Ein Teil der Verfahren wurde gebraucht, um mit den verfügbaren Daten spezifischere Analysen durchzuführen.

Die höchste Stufe der Anwendung statistischer Methoden bestand in der Benutzung von Regressions- und Diskriminantenanalysen. Zunächst soll die Regressionsanalyse erläutert werden. Benutzt wurden multivariate Regressionsanalysen. Ziel war es, die Ausprägung eines Wirklichkeitsmerkmals aufgrund der Kenntnis vieler anderer Wirklichkeitsmerkmale zu schätzen. Dabei wurde mit OLS Schätzungen gearbeitet. Bei Schätzungen einiger der Regressionsgleichungen wurden Dummies gebraucht. Zurückgegriffen wurde dabei auf die Standards, die softwareorientiert vorgegeben waren. Die Regressionsgleichung wurde additiv spezifiziert. Die Regressionsgleichung ist wie folgt definiert (Tabachnick/Fidell, 1989), d. h., die Gleichungen nehmen folgende Form an:

$$
y=a_{1} x_{1}+\ldots a_{n} x+e_{n}
$$

Zeichenerklärung:

$y=$ endogene Variable, $x_{1} \ldots x_{n}=$ exogene Variablen,

$\mathrm{a}_{1} \ldots \mathrm{a}_{\mathrm{n}}=$ Parameter, $\mathrm{e}=$ Stöningsausdruck 
Dabei wurden die Gleichungen auf Multikollinearităt der Variablen geprüft. Gearbeitet wurde mit Erfahrungswerten. Das Vorliegen der Multikollinearität wurde dann angenommen, wenn die Werte der Pearsonkorrelation deutlich über einem Wert von 0.5 lagen. Heteroskedastizitäts- bzw. Fehlspezifikationstests wurden nicht durchgeführt.

Bei den Regressionsanalysen wurden die Betakoeffizienten berücksichtigt. Diese sind wie folgt definiert (SPSS/PC Base Manual; Chicago 1988; S. B-219):

$$
{ }_{\text {BETA }} \pi=B\left[\begin{array}{c}
S \\
R \\
\hdashline \\
Y
\end{array}\right]
$$

$S_{K}$ entspricht der Standardabweichung der k-ten unabhängigen

Variable. Die B-Werte liegen im Spektrum von -1 bis +1 . Betakoeffizienten ermöglichen Schätzungen der relativen Bedeutung der Variablen. Die Schätzungen sind Ausdruck der induktiven Methode.

Die Diskriminantenanalysen wurden ergänzend zu den Regressionsanalysen durchgeführt. Damit sollten die Ergebnisse der Regressionsanalyse analysenspezifisch bestätigt werden. Sie wurden bei den Analysen eingesetzt, in denen die abhängige nominal skalierte Variable dichotom ist. Neben den Diskriminanzwerten wurden in Form der Diskriminanzfunktionen beschrieben, wie sich die beiden Gruppen am besten trennen lassen. In der Klassifikationsmatrix (in der Anlage) wurde dargestellt, inwieweit es der zugrunde liegenden Diskriminanzfunktion gelingt, aufgrund der Kenntnis der Merkmalsausprägungen die Gruppenzugehörigkeit der Untersuchungseinheiten zu schätzen (Patzelt, 1985).

Bei der jeweiligen Diskriminantenanalyse sind folgende Werte vorhanden, die hier definiert werden sollen.

Wilks' Lambda ist folgendermaßen definiert (SPSS/PC + Advanced Statistics V2.0, 1988, S. B-5): "Wilks' lambda, sometimes called U statistic... When variables are considered individually, lambda is the ratio of the within-groups sum of squares to the total sum of squares."

Bevor im empirischen Teil der Arbeit auch die Methodologie der Untersuchung weitere Vertiefung erfăhrt, soll zunächst im folgenden Kapitel auf die untersuchten Organisationen eingegangen werden. 


\section{Die empirisch untersuchten Organisationen}

Ziel dieses Kapitels ist es, die empirisch untersuchten Organisationen kurz vorzustellen. Die dabei verarbeiteten Informationen beruhen auf Interviews, die mit der Zielsetzung durchgeführt wurden, qualitative Informationen zur Ergänzung der quantitaliven Informationen zuerhalten. Dieses Kapitel behandelt die Organisationen hinsichtlich Organisationsstruktur, Strategie und Charakteristiken des internen Arbeitsmarktes. Zunächst wird jede Organisation einzeln vorgestellt, anschließend wird auf Organisationsmerkmale in einer organisationsvergleichenden Sichtweise (Lammers, 1983) eingegangen. Schließlich gilt es zu fragen, inwieweit aus einer ganzheitlichen Sicht Personalführung im Rahmen der sozialen Unternehmensfuhrung typisch ist für eine bestimmte Organisationskonfiguration. Damit sollen die in diesem Abschnitt zur Sprache kommenden Einzelphänomene synthetisien werden.

Geprüft wird dabei, inwieweit sich auf Grund des empirischen Materials typische, beschäftigungsrelevante Anhaltspunkte für die beiden Organisationskonfigurationen ergeben sowie auf deren Grundlage für den intemen Arbeitsmarkt.

\subsection{Das Krankenhaus}

\subsubsection{Historische Entwicklung des Krankenhauses}

Das Krankenhaus wurde 1931 gegründet. Die Initiative ging vom Vorstand eines katholischen Schwestemordens aus. 1967 ging die Krankenhausleitung und das Eigentum am Krankenhaus über an eine Stiftung. Die Einrichtung gilt im Rahmen der provinzialen Funktionsplanung als Zentralkrankenhaus in der Region im Unterschied zu den Kategorien "akademisches Krankenhaus" und "Basiskrankenhaus" (Algemeen jaarverslag, 1987). Für das Zentralkrankenhaus steht ein Einzugsgebiet von nund 150000 Einwohner zur Verfügung. Nach und nach entwickelte sich das Krankenhaus zu einer Einrichtung, die den modernen Erfordemissen der Krankenversorgung Rechnung trägt. So wurden in den sechziger Jahren bauliche Erweiterungen vorgenommen. Die Entwicklung setzte sich in den siebziger Jahren fort und erreichte ihren Höhepunkt Ende der siebziger Jahre durch eine vollständige Renovierung. Anfang der achtziger Jahre konnte ein neuer Gebäudekomplex in Betrieb genommen werden, der mil der funktionalen Erweiterung der Dienstleistungen in Form einer Poliklinik ihren Ausdruck fand. Die Bauaktivitäten wurden im Laufe der Jahre bis zur Gegenwart fortgesetzt. 


\subsubsection{Branche, Aufgabenfeld, Markt und Organisationsumgebung}

Die Organisation "Krankenhaus" ist ein Dienstleistungsunternehmen im Bereich der Gesundheitsversorgung. Das Aufgabenfeld ist auf die regionale Gesundheitsversorgung konzentriert. Der Markt "Gesundheitsversorgung" erstreckt sich auf die verschiedenen Krankenhaustypen. Diese Funktionseinteilung beinhaltet auch eine Arbeitsteilung zwischen den verschiedenen Krankenhauseinrichtungen. Die Organisationsumgebung des untersuchten Krankenhauses ist recht stabil. Dies läßt sich ableiten aus der Nachfrage von Gesundheitsdienstleistungen und den staatlichen Maßstäben der Gesundheitsvorsorge.

\subsubsection{Aufbau des Krankenhauses}

Bei der Betrachtung des Aufbaus der Organisation wird das Organigramm des Krankenhauses zugrunde gelegt. Wir bedienen uns einer "top-down" Perspektive.

Unterhalb der Vorstandsebene ist das Organigramm durch die Aufteilung der Verantwortlichkeit auf Direktionsebene gekennzeichnet. Der medizinische Bereich unterliegt der Zuständigkeit des medizinischen Direktors, der kaufmännische Bereich der des ökonomischen Direktors. 
Abb. 13: Organigramm des Krankenhauses

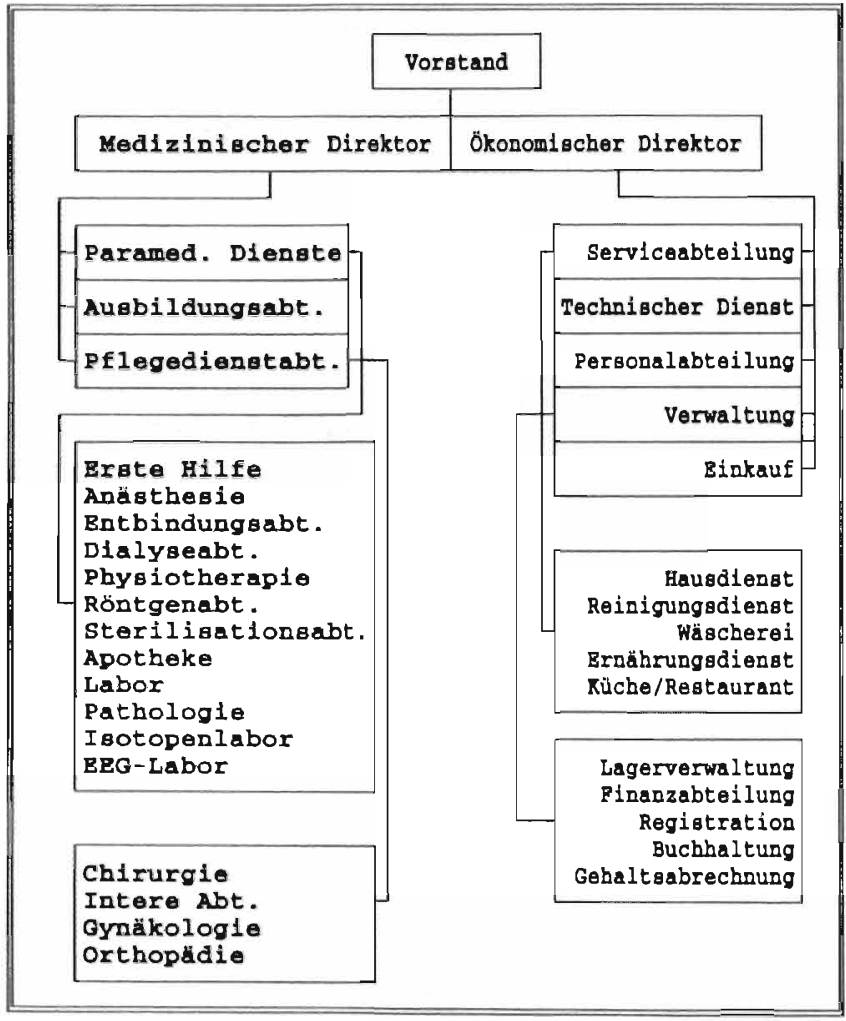

Diesem unterstehen alle nicht-medizinischen Abteilungen. Dazu gehören sämtliche Serviceabteilungen von Küche/Restaurant über Reinigungsdienst bis hin zur Rezeption und Wäschedienst. Daneben existient der technische Dienst mit den verschiedenen Zuständigkeiten. Außerdem die Verwaltung, die ein Spektrum umspannt, das von der kaufmännischen über die Patientenverwaltung bis hin zur Finanzbuchhaltung reicht. Auch die Einkaufahteilung sowie die Personalabteilung fallen unter diese Zuständigkeit.

Der 'medizinischen Direktion' unterstehen Pflegedienstabteilungen, die die Klinik, die Operationsräume und Poliklinik einschließen, um nur einige zu nennen. Ein weiterer Zuständigkeitskomplex besteht in den sogenannten paramedizinischen Diensten, für die hier exemplarisch die Bakteriologie-, die Hygiene- und Röntgenabteilung erwähnt werden sollen. Schließlich bildet die Aus- und Weiterbildungsabteilung ein weiteres Zuständigkeitselement.

Mit dem hier nur grob dargestellten Organigramm läßt sich andeuten, daß eine Vielfalt von Abteilungen eine dezentrale Organisationsstruktur (Buitendam, 1991) repräsentieren. 


\section{I.4 Verteilung der Mitarbeiter auf Mintzbergs "Parts"}

Was die methodologische Perspektive der Einteilung der Parts betrifft, so läßt sich auf Kapitel 5 verweisen. Bei der Einteilung des Personals nach Parts wurden folgende Kriterien berücksichtigt.

Beim "strategic apex" wurden Personen einbezogen, die verantwortliche Leitungsbefugnisse der Organisation verkörpem, so die Direktoren, die in der Hierarchie oben stehen. Beim Part "middle line" wurden alle Abteilungsleiter berücksichtigt. Beim "operating core" wurden alle Personen einbezogen, die im Pflegedienst tätig sind und unmittelbar mit dem Patienten Kontakt haben. Personal, das administrative 'intelligente' Funktionen für die Organisation erfullt, beispielsweise die Personal- und Finanzabteilung, die außerdem zur Standardisierung der Arbeit innerhalb der Organisation beitragen, wurden dem Part "technostructure" zugerechnet. Dienstleistungen, die der Diagnosefindung dienen, beispielsweise Labor und Röntgenabteilung, deren Tätigkeitsfeld nur indirekt auf den Patientenkontakt erstreckt, werden dem 'support staff' zugerechnet.

Ärzte zählen bei diesem Krankenhaus nicht zu den eigentlichen Arbeitnehmern. Es sind freiberuflich tätige Personen, die für das Krankenhaus Dienstleistungen erbringen.

Jede Funktion wurde systematisch auf Tätigkeitsinhalte betrachtet, bevor eine Zuordnung zum jeweiligen Part vorgenommen wurde.

Das Krankenhaus hat 880 Mitarbeiter. Diese verteilen sich über folgende Teile der Organisation (parts): 
Abb. 14: Besetzung der Organisationsteile (parts) des Krankenhauses mit Beschäftigten zum Erhebungszeitpunkt 1989
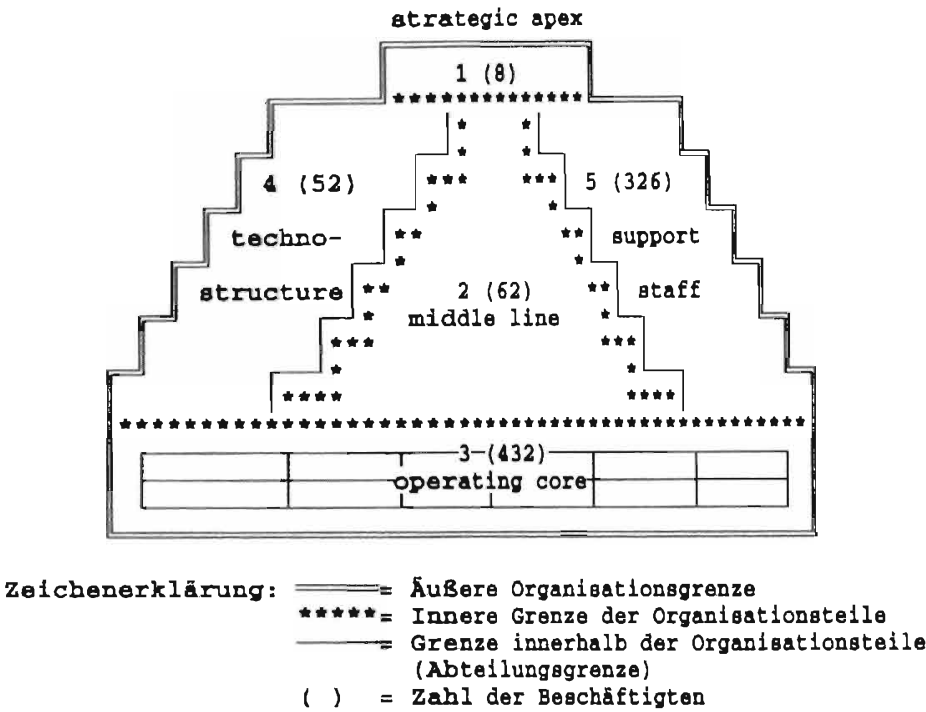

Auffallend ist die starke Besetzung des 'support staff'. Gemeint sind jene Dienstleistungen, die die eigentliche Arbeit am Patienten unterstützen, z. B. Reinigungsdienste, Bibliothek und Pförtner. Dies erklärt sich auf Grund der recht hohen Dienstleistungsaktivität, die als Transferleistung zu anderen Teilen der Organisation aufzufassen ist. Diese GröBenordnung ist für ein Krankenhaus zu erwarten. Support staff und operating core sind die personalintensivsten Teile der Krankenhausorganisation.

\subsubsection{Strategie und Perspektive des Krankenhauses}

Wenn man bei der öffentlichen Einrichtung 'Krankenhaus' von der 'Unternehmensstrategie' (corporate strategy) spricht, geht es um die langfristigen Perspektiven der Organisation. Der Begriff "Unternehmensstrategie" wirkt bei einem Krankenhaus etwas ungewöhnlich, doch wird sich im folgenden zeigen, daß der Gebrauch des Begriffs gerechtfertigt ist (Romme, 1992).

Unternehmensstrategie wird aufgefaßt als Organisationsstrategie. Bei dieser Betrachtung gehen wir über die in der Literatur anzutreffende funktionalistische/rationale Betrachtungsweise hinaus, die darauf beruht, kontinuierlich die in- und extemen Bedingungen einzuschäuen. Hier soll mehr der "logics of organizational action" (Karpik, 1972) Rechnung getragen werden, einem Muster von Entscheidungen und Aktionen, die sich im Laufe der Zeit zu einer Strategie verdichten (Romme et al., 1989). Die Strategie entsteht also aktionsbezogen. Mintzberg/Waters (1985) sprechen von 'emergent strategy' als einem StrategieprozeB. In Bezug auf den intemen Arbeitsmarkt lassen sich verschiedene Dimen- 
sionen von Strategie erkennen. Dabei geht es zunächst einmal um die strategische Entscheidung zugunsten des Beschäfligungssystems. Erst mit zunehmender Institutionalisierung von Besetzungsmustern und den dazu nötigen Regelungen entstehen Lösungen, die die strategische Aktualität abnehmen lassen.

Unternehmensstrategie im Kontext dieser Arbeit (Miles/Snow, 1978) problematisiert drei verschiedene Perspektiven. Zunächst geht es um die strategischen (langfristigen) Entwicklungsperspektiven des Krankenhauses, dann werden Inhalte der strategischen Entscheidungsfindung angesprochen, bevor schlieBlich strategische Probleme in Verbindung mit Human Resources Management zur Sprache kommen.

Was die strategische Entwicklungsperspektive angeht, so zeichnet sich von Seiten des niederländischen Staates ein neues Führungskonzept ab, in dem eine Neuorientierung der provinzialen Krankenhausversorgung angestrebt wird. Die genauen Kriterien der Ausführung sind noch unbekannt. Dieses Führungskonzept beschränkt die strategische Planung. Dennoch zeichnen sich aus der Vergangenheit resultierend Entwicklungen ab, die in den nächsten Jahren noch mehr Bedeutung gewinnen werden.

Seit 1987 wurden Bettenreduzienungen als Folge der Implementierung der Vorschläge der "Kommission Dekker" angekündigt, die offenbar auch die Anfangsphase der 90er Jahre beherrschen werden. Von den 495 Betten des Krankenhauses wurden in einer ersten Reduzierungsaktion 37 Betten aufgegeben. Im Zuge der Neubestimmung der Bettennorm, die bisher bei 3,7 Betten per 1000 Einwohner lag, will man nun zu einer Norm übergehen, die 3,4 Betten per 1000 Einwohner zum Ziel hat. Für das Krankenhaus wurde für 1990 eine maximale Bettenreduktion von 45 geplant.

Die hier nur grob angedeutete Entwicklung dürte zukünftig zu einer stärkeren Arbeitsteilung zwischen den Krankenhäusem in der Provinz führen. Mit dieser Entwicklung wird eine stärkere Kooperation des Krankenhausmanagements verbunden sein.

Strategische Informationen lassen sich nach Auskunft der Krankenhausleitung über folgende Informationsquellen generieren: zum einen ist der nationale Krankenhausrat zu nennen, zum anderen die Praxiserfahrung und schlieBlich der im Zweimonatsrhythmus stattindende Erfahrungsaustausch im Rahmen des Wirtschaftsdirektorentreffens. Diese Informationen gehen sowohl auf persönliche als auch auf unpersönliche Quellen zurück. Die Verantwortlichkeit für die Entwicklung und Formulierung strategischer Führung liegt bei der Direktion.

In nächster Zeit werden Entwicklungen erwartet, die auf Organisationsebene von strategischer Bedeutung sind. Das betrifft zunächst den weiteren Aus- und Umbau des Krankenhauses. Darüberhinaus mit Bezug auf das Personal gilt es, zu einer 'Kräftebündelung' in der Provinz zu kommen was die Ausbildung von Krankenpfleger und Krankenversorgem betrifft. Allerdings sind die Erfolgsaussichten für diesen Schritt nicht sehr günstig, da die erwartete Resonanz in den zuständigen Gremien bisher ausgeblieben ist. Werbeaktivitäten mit dem Ziel, Menschen für eine Ausbildung im Krankenhaus zu gewinnen, sind angelaufen. 
Ein weiteres Feld ist die Personalfunktions- und Kapazitätsplanung, die unter anderem das Ziel verfolgt, Mitarbeitem die Chance des intemen Aufstiegs zu geben. Ein weiteres Anliegen ist die Mechanisierung und Automatisierung sowie die damit verbundenen Investitionen in arbeitssparende Technologien.

In Kooperation mit der Personalabteilung werden einmal pro Woche Personalführungsfragen auf die Tagesordnung gesetzt. In diesem Rahmen ergeben sich die folgenden langfristig zu lösenden Probleme:

- das Problem der Zusammenfassung der Ausbildung von Krankenpflegem und Krankenversorgem;

- das Problem, hoch qualifiziertes lechnisches Personal zu werben, zum Beispiel Genlaboranten und Elektroniker;

- das Problem, mehr Konkurrenz intern zu erzeugen, vor allem im Hinblick auf die Gehaltsstruktur. Als Zielperspektive wären mehr finanzielle Anreize zu schaffen und das Vergütungssystem auf Marktgegebenheiten auszurichten.

\subsubsection{Merkmale der organisationsspezifischen Beschäftigungssituation}

In diesem Abschnitı soll an Hand von Merkmalen auf die Beschäftigungssituation eingegangen werden. Angesprochen wird der Personalaufbau, Weiterbildungs- und Beförderungspraxis, die Personalphilosphie, die Rekrutierungspraxis und die Struktur der Funktionsketten. Viele der hier angesprochenen Beschäftigungsmerkmale zeigen Übereinstimmung mit der von Sehringer (1988) untersuchten vergleichbaren Einrichtung mit 800 Beschäftigten in der Bundesrepublik.

\subsubsection{Zum Personalaufbau des Krankenhauses}

Im Gegensatz zu den noch vorzustellenden Organisationen gilt das Krankenhaus als "junge" Organisation. Mehr als die Hälfte aller Beschäftigten liegt in der Altersgruppe von 20-29 Jahren. Was die durchschnittliche Tätigkeitsdauer betrifft, so ist diese mit einer Besetzung von rund 30 Prozent für den Zeitraum 5 bis 10 Jahre am stärksten ausgeprägt. Die geschlechtsspezifische Verteilung zeig! auf, daß die Organisation zu 75 Prozent aus Frauen besteht.

\subsubsection{Aus- und Weiterbildung sowie Beförderung}

Aus- und Weiterbildung innerhalb und außerhalb der Organisation erfüllen eine wichtige Funktion innerhalb des Krankenhauses. In diesem Abschnitt wird deshalb eingegangen auf die Bedeutung der Aus- und Weiterbildung. Weiterhin verdienen die Beförderungsaktivitäten innerhalb der Organisation Aufmerksamkeit, die vielfach direkt mit der Aus- und Weiterbildung zusammenhängen. Über die Ausbildungsaktivitäten im Krankenhaus existiert gleichzeitig ein wichtiges Personalrekrutierungsinstrument. Dies gitt in besonderem Maße für die Pflegedienstausbildung, die sich über einen Zeitraum von drei Jahren erstreckt. Im Rahmen dieser Ausbildung wird das Ausbildungsziel "Pflegedienstdiplom A" 
angestrebt. Jedes Jahr werden rund 30 Personen für diese Ausbildung rekrutiert. Weitere Ausbildungsrichtungen sind die Ausbildung zum Operationsassistent, die Ausbildung zum Dialysepfleger/-pflegerin, zum neurophysiologischen Laborant und zum radiologischen Laborant. Bei allen Ausbildungsabschlüssen handelt es sich um staatlich anerkannte Berufsabschlüsse.

Darüberhinaus werden zahlreiche Kurse teils intern, teils extern organisiert mit unterschiedlichen Zielrichtungen. So nahmen im Jahr 198760 Krankenpfleger an einem Wiederholungskurs "Wiederbelebung" teil. Außerdem fanden Weiterbildungskurse statt, die das Ziel der Spezialisierung (Onkologie, Intensivpflege, Gynäkologie) verfolgten.

Beförderungen innerhalb des Krankenhauses sind vielfach Ausdruck der Übernahme in ein Beschäftigungsverhältnis nach erfolgter und erfolgreicher Ausbildung. Daneben sind Beförderungen an der Tagesordnung als Ausdruck der Bewährung auf einer bestimmten Funktion aber auch als Motivationsinstrument. In den letzten Jahren verzeichnet die Organisation kein Beschäftigungswachstum. So sind durch die langfristige Besetzung der Stellen in bestimmten Funktionen relativ wenig Aufstiegsmöglichkeiten gegeben. Dennoch ist von den potentiellen Aufstiegsmöglichkeiten eine deutliche Funktionshierarchie entwickelt, die den professionellen beruflichen Werdegang markieren. Aufgrund der tendenziellen Beschränkung eines vertikalen Aufstiegs bleibt vielfach nur der Weg der interorganisationalen Mobilität, d. h. der Wechsel des Arbeitgebers zur Realisierung von Karriereschritten auf Initiative der betroffenen Personen übrig.

\subsubsection{Personalphilosophie innerhalb des Krankenhauses}

Die Personalphilosophie kommt zum Ausdruck als Teil der Organisationsstrategie und läßt sich als 'Personalführung' identifizieren. Die Zielsetzungen sind auf zwei Ebenen anzutreffen: einerseits sind sie im Tarifvertrag für das Krankenhauswesen (C. A. O.; Statuut Sociaal Beleid) anzutreffen, andererseits kommen sie als Personalführungsgrundsätze organisationsspezifisch zum Ausdruck.

In der Natur der Tarifvertragszielsetzungen liegt es, daß diese global formuliert sind. Sie lassen sich in drei Punkten zusammenfassen:

- aus dem Geist der Mitmenschlichkeit die Patienten so gut wie möglich untersuchen, begleiten, betreuen und versorgen;

- Spielraum, Mitbestimmung und Entwicklungsmöglichkeiten schaffen und die Arbeitsmöglichkeiten für alle Arbeitnehmer der Einrichtung zu fördem;

- die Optimierung des Einsatzes der zur Verfügung stehenden Hilfsmittel.

Die Personalführungsgrundsäıze sind organisationsspezifisch formuliert, jedoch bedürfen sie weiterer Operationalisierung.

Die Organisation steht im Zeichen der folgenden konkretisierten Zielsetzungen: 
- Arbeitsorganisation und -klima so zu gestalten, daß die Arbeitnehmer im Geiste der Solidarität ihren Beitrag leisten zum Wohle der Einrichtung/Organisation;

- den Arbeitnehmern der Einrichtung Arbeitszufriedenheit zu vermitteln;

- im Rahmen der Möglichkeiten gute materielle Voraussetzungen für die Arbeitnehmer zu schaffen;

- mitzuwirken an der Entfaltung von Arbeitnehmem in ihrer Funktion unter Berücksichtigung der Neigungen und Fähigkeiten sowie der Möglichkeiten der Organisation, wobei der ältere Arbeitnehmer besondere Aufmerksamkeit verdient.

Soviel zu den Zielsetzungen, inwieweit diese Zielsetzungen realisiert sind bzw. Mitarbeiter sich mit diesen Zielen identifizieren, war nicht Gegenstand der Untersuchung. Dennoch hat sich auf der Basis der gewonnenen Informationen der Eindruck bestätigt, daß ein hohes $\mathrm{Maß}$ an Zielerreichung hinsichtlich der oben genannten Ziele festzustellen ist.

\subsubsection{Rekrutierung und Personalselektion}

In diesem Abschnitt geht es um die Praxis der Besetzung offener Stellen, die Rekrutierung von Personal bzw. Personalselektion. In diesem Abschnitt wird an Hand der empirischen Gegebenheiten geprïft, ob eine der von Windolf/Hohn (1984) empirisch ermiltelten Rekrutierungsstrategien (Kapitel 3) hier aufgrund der organisationsspezifischen Gegebenheiten zuriickzufinden ist.

Offene Stellen entstehen dann, wenn Personal die Organisation verläßt, außerdem in den Fïllen, wo durch Arbeitsunfähigkeit und lange Abwesenheit Ersatzlösungen angebracht erscheinen. Darüberhinaus entstehen offene Stellen durch die Ausbreitung des Personalbestandes. Entwicklung und Aufkommen von offenen Stellen innerhalb der Organisation ist eine gesonderte Analyse gewidmet, die in Kapitel 5 kurz vorgestellt wurde. Vertieft wird die Analyse in Kapitel 8 als Gegenstand der quantitativen Untersuchung.

Folgender 'Verfahrensablauf' bei Entstehung der offenen Stelle wird praktiziert. Die 'Budgetkommission' (bestehend aus Direktion und Personalchef) wird bei Entstehung einer offenen Stelle informiert. Sie trifft die Entscheidung bezüglich der möglichen Besetzung der Stelle.

Entscheidungen über die Besetzung von Stellen, die in der 'Budgetkommission' getroffen werden, betreffen Personal mit befristeter und unbefristeter Anstellung. Die Einstellung von Leiharbeitnehmem erfolgt unter der Regie der Personalabteilung, jedoch mit Zustimmung der Direktion. Eine weitere Personalkasegorie umfaßt die sogenannten Abrufkräfte, Arbeitnehmer, die unter bestimmten Umständen oder im Bedarfsfall telefonisch 'abgerufen' werden.

Ein Kennzeichen des organisationsspezifischen Arbeitsmarktes ist, daß die Stellenwerbung zunächst intern erfolgt (Sehringer, 1988) durch die Personalabteilung. Die offenen Stellen werden organisationsintem ausgeschrieben und bekannt gemacht. Interne Kandidaten 
haben zwei Wochen nach Bekanntgabe der Ausschreibung Gelegenheit, sich zu bewerben. Vom Grundsatz der intemen Ausschreibung weicht die Personalabteilung dann ab, wenn deutlich ist, daß interne Kandidaten vonvornherein nicht zur Verfügung stehen. Für den Fall, daß keine geeigneten Kandidaten intern verfügbar sind, gilt die Aufmerksamkeit früheren Ausschreibungen, um aus dieser Quelle geeignete Bewerber zu finden. Ist dies nicht der Fall, so wird eine Zeitungsanzeige formuliert, die sich an organisationsexteme Personen richtet. In dieser Phase wird das Arbeitsamt dann für Vermittlungszwecke eingeschaltet.

Darüberhinaus zeigte sich, daß es bei der Krankenhausorganisation in geringem Umfang Anzeichen für einen erweiterten organisationsspezifischen Arbeitsmarkt gibt. Dieser kommt dadurch zum Ausdruck, daB organisationsexteme Personen von internen Ausschreibungen Kenntnis nehmen und sich auf intern ausgeschriebene Stellen bewerben. Dies erklärt auch die Entstehung eines 'intemen Portefeuilles', das sich zum einen aus intemen Bewerbungen organisationsextemer Personen aber auch aus sogenannten 'Blindbewerbungen' externer Personen speist.

Die Auswahl intemer Kandidaten erfolgt koordiniert durch Mitarbeiter der Personalabteilung und Mitarbeiter der betroffenen Abteilung. Neben den Bewerbungsgesprächen wird ein Selektionsbeurteilungsformular benutzt, in dem Auftreten und Verhalten, Fähigkeiten, körperliche Erscheinung, Persönlichkeit, Arbeitseinstellung und allgemeiner Eindruck in einer Ja/Nein-Beurteilung operationalisiert wird, das später in einer zusammenfassenden Beurteilung mündet. Nach den Gesprächen mit den Kandidaten werden die gewonnenen Eindrücke ausgetauscht und Details des Übergangs bestimmt, soweit der Kandidat als geeignet erscheint. Die Personalentscheidung wird durch die Personalabteilung dem Kandidaten bekannt gegeben und später auch organisationsintern bekannt gemacht.

Die Auswahl extemer Kandidaten erfolgt über eine Vorselektion durch die Personalabteilung, die dann im Rahmen einer Abteilungsleiterbesprechung weiter konkretisiert wird. Beläuft sich die Zahl der Bewerbungen auf über 20, dann wird eine Auswahl von maximal 12 Kandidaten vorgenommen. Von diesem Muster weicht man ab im Fall der Auswahl von Auszubildenden für Pflegeberufe. Nach der Vorselektion werden Absprachen zwecks Einladung zu Vorstellungsgesprächen gemacht. Das weitere Selektionsverfahren entspricht dann weitgehend dem Verfahren, das für die Auswahl intemer Kandidaten gilt. Im letzten Stadium des Selektionsverfahrens werden mit Zustimmung des Kandidaten Referenzen eingeholt. Eine psychologische und medizinische Eignungsuntersuchung können Teil des Auswahlverfahrens ausmachen.

Für die schnelle Stellenbesetzung für Funktionen mit geringer Qualifikation wurde ein Instrument geschaffen, das bemerkenswert erscheint. Außerhalb der üblichen Reknutierung und Selektion von Mitarbeitern wird mit sogenannten "Aufrufkräften" gearbeitet. Dies sind Personen, die der Personalabteilung bekannt sind und telefonisch zum Arbeitseinsatz 'aufgerufen' werden. Diese Arbeitskräfte sind deutlich kostengünstiger, da sie nicht dem Tarifvertrag unterliegen. Von Seiten der Krankenhausorganisation besteht auch in diesen Fällen nicht die Verpflichtung, Personen im Falle des Aufrufs mit einem bestimmten Zeitkontingent an Beschäftigung zu versehen. Diese Form der Beschäftigung eröffnet alle 
Möglichkeiten eines flexiblen Personaleinsatzes mit selektiven Inanspruchnahmen aber auch Selektivität in der Person des 'aufgerufenen Arbeitnehmers'.

Hier handelt es sich um einen "spotmarket". Allerdings ist zu bericksichtigen, daß der Umfang der Inanspruchnahme gering ist. So betrug der Einsatz des "Aufrufpersonals" 1987 nur 1,4 Prozent bezogen auf die Arbeitszeit der fest eingestellten Mitarbeiter auf Jahresbasis.

Wie schon in Kapitel 3 angedeutet, bestehen verschiedene Rekrutierungsstrategien, die in der Realität selten idealtypisch anzutreffen sind. Mehrere der von Windolf/Hohn abgeleiteten Rekrutierungsstrategien treffen hier zu. Dies gilt für die 'Status-quo-Strategie', die idealtypisch die Rekrutierungsstrategie repräsentiert, die für den intemen Arbeitsmarkt zutrifft. Kennzeichen dafür ist die Personalsuche aufgrund von informellen Netzwerken (Boxman, 1992). Die Suche konzentriert sich dabei auf Familienmitglieder oder Bekannte der Personen, die in der Organisation beschäftigt sind. Aufgrund der Dominanz von Personal mit Fachqualifikation ist diese Rekrutienungsstrategie vorwiegend für Personal im Bereich des 'support staff' zutreffend. Abgerufen werden mit dieser Strategie allgemeine bzw. Jedermann-Qualifikation(en). Eine weitere Rekntierungsstrategie, die 'autonome Strategie' gilt vorwiegend für die Beschaffung von Personal mit Fachhintergrund, was typisch ist für die Organisationsform der professionellen Bürokratie. Kennzeichend ist eine sorgfältige Auswahl innerhalb eines mehrstufigen Auswahlverfahrens sowie der systematische Vergleich von Anforderungs- und Persönlichkeitsprofil. Diese Rekrutierungsstrategie dominiert deutlich. Mit der Rekrutienung von 'Aufrufpersonal' in geringem Umfang will man offenbar Gebrauch machen von der flexiblen Strategie, die keine großen Anforderungen bei der Personalauswahl stelit. Im Vordergrund dürte eine schnelle Allokation durch 'Abruf' stehen.

Zusammenfassend ausgedrückt dominien beim Krankenhaus die 'autonome Rekrutierungsstrategie' flankiert durch die 'Status-quo-Strategie' bzw. die 'flexible Strategie'.

\subsubsection{Funktionsketten innerhalb der Krankenhausorganisation}

Funktionsketten lassen sich definieren als zusammenhängende Funktionen (Baron, et al., 1986), die einen potentiellen Mobilitätspfad innerhalb der Organisation vorgeben (siehe auch Kapitel 3). In den meisten Fällen ist der Mobilitätpfad auf Abteilungen reduziert. Funktionsketten sind durch unterschiedliche Länge charakterisiert. Als Tendenz läßt sich beim Krankenhaus die "Pflegedienstfunktionskene" als relativ lange Funktionskette bezeichnen, während Unterstützungsfunktionen, also klassische "suppont-staff-Funktionen" relativ kurze Funktionsketten aufweisen.

Die systematische Erfassung der Lokalisation und Verteilung bzw. die innerorganisatorischen Grenzen von Funktionsketten führt zu einer ungeahnten Komplexität (Baron, u. a.; 1986). Exemplarisch sollen hier zwei Funktionsketten vorgestellt werden, wovon eine sich innerhalb eines Organisationsteils (par) erstreckt, die andere zwei Organisationsteile erfaßt. Kriterium für die Einteilung und die Vergleichbarkeit ist das Gehalt bzw. Gehaltsniveau. 
Abb. 15: Funktionsketten im Krankenhaus

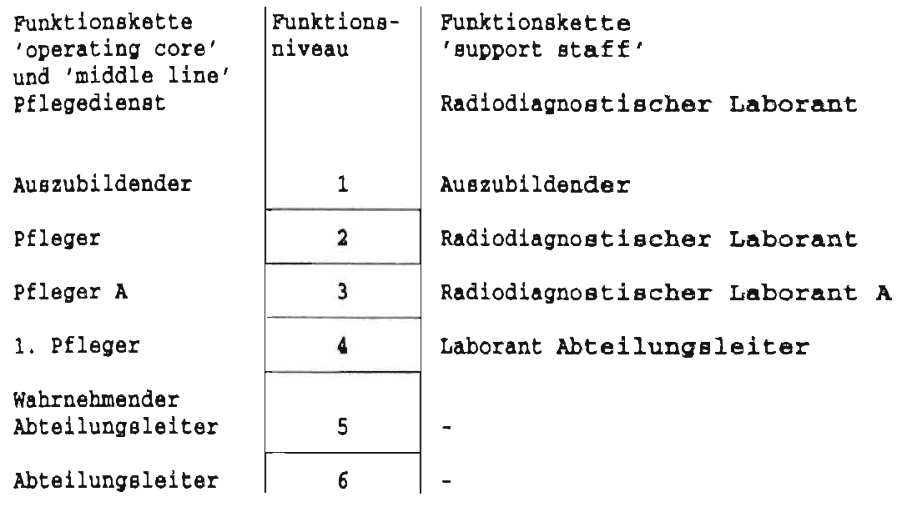

Die Abbildung zeigt deutliche Unterschiede des Funktionsniveaus bzw. der Länge der Funktionsketten in den hier zum Vergleich herangezogenen Organisationsteilen. Die Funktionsbezeichnungen 5 und 6 (middle line) bilden die Fortsetzung der Laufbahn auf dem Niveau der 'middle line'. Dagegen bleibt die Funktionskette 'support staff' auf Niveau 4 als höchste Stufe der Funktionskette beschränkt. Im Rahmen der empirischen Analyse wird sich zeigen, daß die potentielle Mobilität über Funktionsketten innerhalb des 'support staff' beschränkt ist. Demgegenüber steht ein erweitertes, partübergreifendes Mobilitätsfeld von 'operating core' nach 'middle line'. Allein dieses Beispiel läßt erkennen, welche Komplexität auch unter Berücksichtigung der Gestaltungsaspekte auf mikrowrganisatorischer Ebene anzutreffen sind.

\subsection{Die Sozialversicherung}

Die Sozialversicherung ist ein Dienstleistungsuntemehmen, das überwiegend beschäftigt ist mit der Auslegung und Anwendung von Sozialversicherungsgesetzen. Auch diese Organisation soll im folgenden kurz vorgestellt werden.

\subsubsection{Historische Entwicklung der Sozialversicherung}

Die Sozialversicherung wurde 1952 gegründet zu einer Zeit, in der wichtige Änderungen in der Ausführung sozialer Gesetze in den Niederlanden stattgefunden haben. Der gesetzliche Auftrag wurde von Seiten des Staates zur administrativen Ausführung der Sozialversicherung den sogenannten "Betriebsvereinigungen" überlassen, die die Sozialversicherungsverwaltung begründeten. Diese befaßt sich mit der Anwendung und Implementierung von Gesetzen auf der Grundlage des Krankenversicherungs-, des Arbeitslosen- und Arbeitsunfähigkeitsgesetzes. Die damit verbundenen Dienstleistungen beschränken sich auf Arbeitnehmer, die in einem betrieblichen Arbeitsverhältnis stehen. Die Sozialversicherung gilt also nicht für Arbeitsverhältnisse des öffentlichen Dienstes.

Zum Aufgabenpaket der Sozialversicherungsverwaltung gehört die medizinische Abteilung sowie die Betriebsrentenverwaltung und die sogenannten VUT-Stiftungen, eine Einrich- 
tung, die die finanzielle Verwaltung der Vornhestandsregelung übernimmt. In der Bundes republik Deutschland wird jeweils ein Teil dieser Aufgaben durch die Arbeitsmarktverwaltung, Rentenversicherungen und Berufsgenossenschaften durchgeführt.

Konkret umfassen die Aufgaben der Sozialversicherungsverwaltung das Erheben von Prämien, die Festellung des Anspruchsrechts und die Ausbezahlung von Geldleistungen an die Betroffenen. Während die Prämienerhebung zentral in der Hauptverwaltung in Amsterdam geschieht, sind die anderen Aufgaben verteilt auf 30 "Distrikt- und Regionalverwaltungen". Eine dieser Regionalverwaltungen war Gegenstand der Untersuchung.

\subsubsection{Branche, Aufgabenfeld, Markt und Organisationsumgebung}

Die Sozialversicherung nimmt Aufgaben wahr in einer stabilen Organisationsumgebung. Von der Organisationsumgebung gehen Einflüsse auf die Organisation aus. Dazu gehört der "Versicherungsrat" (ein Beratungsgremium), die Betriebsvereinigungen, die Hauptverwaltung sowie verschiedene Interessensgruppierungen. Ein wichtiger Einfluß geht auf Grund der Gesetzgebung vom Staat aus. Nicht zu unterschäızen sind die eigentlichen "Kunden" der Sozialversicherung: die Anspruchsberechtigten.

Als zentrale Zielsetzung der Sozialversicherung gegenüber den Anspruchsberechtigten ist die Feststellung der richtigen Höhe der Unterstützung und deren Ausbezahlung. Daneben steht Gewährung von Information an die Anspnuchsberechtigten im Vordergrund.

Der Aufbau der Sozialversicherung ist geregelt im "Organisationsgesetz der Sozialversicherung". Dies schreibt den organisatorischen Aufbau vor und die Aufgaben der Versicherung. Allein der Sozialminister ist berechtigt, Anweisungen zu erteilen, die zusätzliche Aufgaben betreffen. Gesetzlich festgelegt ist auch, daß die Sozialversicherung ohne Gewinn arbeitet. Der Vorstand ist parilätisch besetzt aus Arbeitgeber- und Arbeitnehmervertreter.

Obwohl vom Umfang der Aufgaben, Strategie der Organisation und Organisationsumgebung die Sozialversicherung als öffentliche Einrichtung bezeichnet werden kann, so wird dies von der Leitung in Frage gestellt. Diese ist nach dem eigenen Selbstverständnis eine private Einrichtung, die von den Betriebsvereinigungen einzelner Branchen getragen wird. Daraus wird deutlich, daß es um eine Selbsthilfeeinrichtung geht, die einen quasi-öffentlchen Charakter hat. Wir gehen nach eingehender Bewertung interner und externer Organisationskarakteristiken davon aus, daß es sich um eine öffentliche Einrichrung handelt auf Grund der spezifischen Anwendung von Gesetzen als Gegenstand des Tätigkeitsfeldes, auf Grund der typischen Konstruktion als Auftragsverwaltung, die der staatlichen Kontrolle unterliegt und auf Grund der konkurrenzfreien stabilen, monopolartigen und marknuabhängigen Stellung.

\subsubsection{Authau der Sozialversicherungsorganisation}

Die Organisitionsstruktur umfaßt die Leitung der Einrichtung, verschiedene Stabsstellen sowie die komplexe Struktur von mittleren und unteren Hierarchiebereichen. 
Die Leitung besteht aus der Person des Direktors, seines Stellvertreters und einem beratenden Arzt. Unter der Verantwortichkeit des stellvertretenden Direktors faillt die Ausfíhrung von Gesetzen zur Arbeitslosen-, Kranken-, Renten- sowie Witwen- und Waisenversicherung. Daneben fällt in seine Zuständigkeit die "Einspruchsabtéilung" sowie der Aubendienst, die Regionsaußenstelle neben der Verwaltung des "medizinischen Dienstes". Nach außen gerichtet erstreckt sich seine Aufgabe auf die Kommunikation mit der "Außerwelt", Als Stabsstelle organisiert ist die Personalabteilung.

Abb. 16: Organigramm der Sozialversicherung

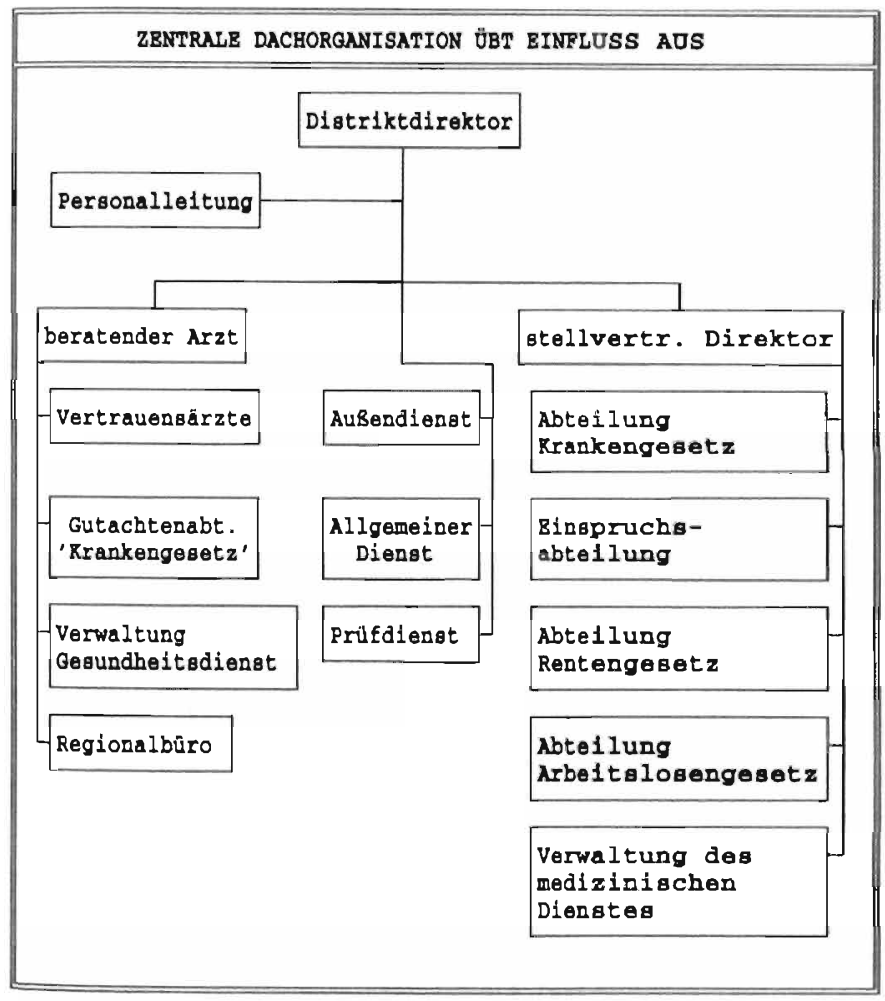

Zwecks allgemeiner Besprechung und Koordinierung der Aufgaben kommen wöchentlich einmal Direktion und Abteilungsleiter zusammen. Täglich findet eine Beratung zwischen Direktor und seinem Stellvertreter statt. Zwischen den unterschiedlichen Abteilungen wird ebenfalls kommunizien, wenn nötig auch informell. Innerhalb der Abteilungen wird einmal wöchentlich eine Besprechung abgehalten, wobei man den unterschiedlichen Hierarchieniveaus Rechnung trägt. Auf die Abteilungsstruktur wird bei späterer Gelegenheit eingegangen. 


\subsubsection{Verteilung der Mitarbeiter auf Mintzbergs "Parts"}

Die Sozialversicherung hatte zum Zeitpunkt der Untersuchung 365 Mitarbeiter. Diese Organisation zeichnet sich in den letzten Jahren aus durch eine kontinuierliche Personalexpansion.

Die Bestimmung der Parts setzt sich wie folgt zusammen: dem "strategic apex" wurden der Direktor, sein Stellvertreter sowie der leitende Arzt zugerechnet. Die "middle line" spricht in erster Linie Abteilungsleiterniveau an. "Operating core" umfaßt die Arbeimehmer, die mit der Ausführung und Anwendung von Gesetzen befaßt sind. Der Personalleiter sowie ein Mitarbeiter, der mit Kontrollaufgaben beschäftigt ist, zählen zu der schwach entwickelten "technostructure". Schreibkräfte und andere Personen, wie z. B. Vertrauensärzte bilden in der Hauptsache den Pan "support staff". Zum Untersuchungszeitpunkt ergab sich folgende Personalverteilung nach Parts:

Abb. 17; Besetzung der Organisationsteile (parts) der Sozialversicherung mit Beschäftigten zum Erhebungszeitpunkt 1989
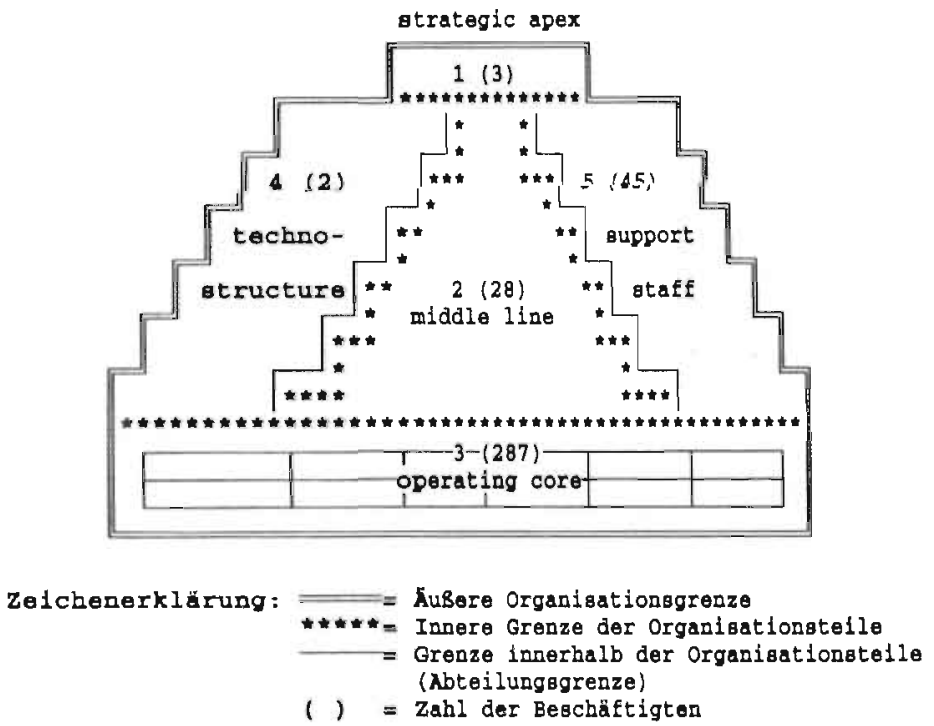

Auch bei dieser Organisation konzentrieren sich die Beschäftigten auf den Organisationsteil 'operating core'. Relativ schwach ausgeprägt ist qua Personalbesetzung die 'middle line'. Der Organisationsteil 'technostructure' ist in Anbetracht der Wahmehmung wichtiger Dienstleistungen durch die Zentrale in Amsterdam nahezu 'unterentwickelt'. 


\subsubsection{Strategie und Perspektive der Sozialversicherung}

Aus der Strategieuntersuchung von Romme et al, 1989; 1990, die ebenfalls diese Organisation zum Untersuchungsgegenstand hatte, läßt sich ableiten, daß die Sozialversicherung deutlich einer Effizienzkostenstrategie und weniger einer Flexibilitătsstrategie folgt. Die Organisation befindet sich wie gesagt in einer stabilen Umgebung. Von Seiten der Organisation wird so gut wie kein Risiko eingegangen. Dieses Ergebnis läßt sich bestätigen als Resultat des Strategieprozesses. Danach zeigt sich ein hoher Grad von Übereinstimmung in Handlungsabsichten innerhalb der Organisation.

Doch weist Romme et al. (1990) darauf hin, daß diese Erscheinung vor dem Hintergrund $2 u$ sehen ist, daß es sich um die Intemalisierung der von außen auferlegten Beschränkungen handelt. Die Strategie der Sozialversicherung ähnelt innerhalb der von Mintzberg und Waters (1985) entwickelten Strategietypologie der des "auferlegten" (imposed) Typus.

Die strategische Vision, die innerhalb der Hauptverwaltung der Sozialversicherung entwickelt wurde, geht aus von einem unter Druck stehenden Markt der "Ausführung von Sozialversicherungen". Dabei gilt es, die bisher "vorteilhafte Marktposition" der Organisation zu wahren. Markt als Schlüsselbegriff steht auch als Perspektive für die Einführung von mehr "Markt" innerhalb der Organisation. Die Organisation wird momentan beherrscht von Reorganisationsaktivitäten, die in der Neuverteilung von Aufgaben ihren Ausdruck finden. Sichtbar wird dies exemplarisch an der elektronischen Arbeitszeitregistration und in der Rekrutierung eines Personalmanagers, unter dessen Federführung die Umgestaltung stattfindet. Offiziell heißt es, daß die interne Struktur "angepaßt" werden muB.

Die Organisationsentwicklung wird intern so gesehen, daß man sich in Richtung einer offenen Organisation zubewegt, die 'sensibel' ist für Signale, die von außen kommen. Dazu gehört auch die Verstärkung der "Kundenorientierung" sowie der Arbeitsqualität. Mit dem nötigen Kostenbewußtsein will man zu einer veränderten Organisationskultur kommen.

\subsubsection{Merkmale der organisationsspezifischen Beschäftigungssituation}

In diesem Abschnitt gilt es, die organisationsspezifische Beschäftigungssituation $\mathrm{zu}$ betrachten. Gegenstand dieses Abschnitts ist der Personalaufbau, die Aus- und Weiterbildung, die Personalphilosophie, Rekrutierung und exemplarisch die Betrachtung einer Funktionskette.

\subsubsection{Zum Personalaufbau der Sozialversicherung}

Der Betrachtung des Personalaufbaus liegen die tabellarischen Übersichten "Entwicklung des Lebensaltersaufbaus", die "durchschnittliche Tätigkeitsdauer von Arbeitnehmem" sowie die "Verteilung von Männern und Frauen" zugrunde, die am Ende dieses Kapitels Gegenstand einer ausführlicheren Betrachtung sind.

Im Vergleich zum Krankenhaus ist die Besetzung der höheren Lebensalterskategorien bei der Sozialversicherung deutlich stärker entwickelt. Das Durchschnittsalter der Beschäftigt- 
en der Sozialversicherung liegt über dem des Krankenhauses. Das Zahlenmaterial über die durchschnittliche Tảtigkeitsdauer bestätigt die oben ausgedrückte Tendenz. In der Sozialversicherung gibt es mehr langfristige Beschäftigungsverhältnisse als im Krankenhaus. Eine Erklärung dafür bietet die Geschlechterverteilung innerhalb der Organisation. Diese ist relativ ausgewogen. Rund 55 Prozent Männer stehen einem Anteil von 45 Prozent Frauen gegenüber.

\subsubsection{Aus- und Weiterbildung sowie Beförderung}

Neu eintretende Mitarbeiter bekommen eine betriebsspezifische Ausbildung in Form des "leaming on the job". Nach erfolgreichem AbschluB erfolgt in der Regel eine Gehaltsaufbesserung. In zunehmenden Maße werden Mitarbeiter zum Besuch interner Kurse geschickt. Weitergehende Ausbildungsvorhaben werden an einer intemen Schulungseinrichtung in Form von Kursen angeboten, zu denen die Regionalverwaltungen ihre Mitarbeiter freistellen. Auf Abteilungsniveau werden Weiterbildungskurse organisationsintem angeboten und durchgeführt, die unmittelbar aufgabenbezogen sind. Eine eigene Aus- und Weiterbildungsabteilung besteht zur Zeit nicht. Sie erscheint auch nicht als Notwendigkeit, da die beruflichen Lemaktivitäten der Mitarbeiter stark an die Art der Tätigkeiten gekoppelt sind.

Die Möglichkeiten der Beförderung orientieren sich an der Erfahrung, die die Mitarbeiter innerhalb des Arbeitsgebietes gesammelt haben. Es gibt keinen Beförderungsautomatismus, doch sind den Möglichkeiten des intemen Aufstiegs organisatorische Grenzen gesetzt, auf die im empirischen Teil der Arbeit näher eingegangen werden wird. Mitarbeiter können sich intern auf freiwerdende Stellen bewerben, die dann auch von innen möglicherweise wiederbesetzt werden. In der Vergangenheit, d. h. 1987 ergaben sich vorübergehend viele offene Stellen auf höheren Funktionsebenen, da viele ältere Mitarbeiter fast gleichzeitig von der Vorruhestandsregelung (VUT) Gebrauch machten. Diese Erscheinung beförderte kurzfristig die intraorganisationale Mobilität durch das inteme Nachrücken von Mitarbeitern auf freigewordenen Stellen.

\subsubsection{Personalphilosophie innerhalb der Sozialversicherung}

Ein Aspekt der 'Personalphilosophie' in der Organisation ist darauf gerichtet, "Elemente des Marktes" organisationsintem zur Geltung zu bringen. Dies führt zu Konsequenzen auch für die Personalführung. Es wird deutlich, daß von außen auferlegte Vorstellungen die bestehende Organisation verändem (Paauwe, 1989). Während bisher die Organisation expandierte, steht nun alles im Zeichen der Reorganisation. Bis 1992 ist ein Abbau von 15 Prozent der Beschäftigten geplant.

Eine ausgesprochene Personalphilosophie besteht nicht. Die Personalabteilung, die drei Personen umfaßt, bestand bis vor kurzem noch nicht. Personalangelegenheiten waren beschränkt auf Personalverwaltung in der Zuständigkeit des stellvertretenden Direktors. Der Aufbau der Personalabteilung steht in deutlichem Zusammenhang mit Automatisierungsvorhaben und Reorganisation. 
Bisher wurde kein extensiver Gebrauch von Personalführungsinstrumenten gemacht. Aus den Geprächen mit der Leitung der Organisation geht hervor, daß Personalplanung und Laufbahnentwicklung eingeführt werden. Auch die organisationsinterne Dokumentation von Personalinformationen erfolgte in der Vergangenheit unregelmäßig und keinesfalls nach einheitlichen Kriterien. Dem wurde bei der Datenerfassung dieser Untersuchung Rechnung getragen durch die Heranziehung alternativer Informationsquellen. Inzwischen wurde ein Personalinformationssystem implementiert, das die oben genannte Zielsetzung der Personalplanung und Laufbahnentwicklung unterstützen wird.

\subsubsection{Rekrutierung und Personalselektion}

Die Rekrutierung von Personal folgt den klassischen Mustern des internen Arbeitsmarktes. Dies bedeutet, daß Rekrutierungen via die "ports of entry" meistens auf unterem Hierarchieniveau stattfinden. Über eine gut entwickelte Hierarchie von Funktionen wird es den eintretenden Mitarbeitem ermöglicht, intern aufzusteigen. Mitarbeiter mit akademischer Ausbildung, die mit Führungsaufgaben befaßt sind, werden auf dem externen Arbeitsmarkt rekrutiert. Das Prinzip der intemen Rekrutienung ist hinsichtlich der Leitung der Organisation außer Kraft gesetzt. Durch eine innerorganisatorische Ausbildung wird eintretenden Mitarbeitem, die Möglichkeit geboten, eine betriebsspezifische Ausbildung zu erhalten, eine Voraussetzung, um innerorganisatorisch aufzusteigen. Freie Stellen werden zunächst intern ausgeschrieben bevor extern geworben wird.

Bei der Sozialversicherung ergeben sich Anhaltspunkte für einen erweiterten internen Arbeitsmark! (Sehringer, 1988; Deeke/ Fischer, 1986), der Rekrutierungen im Lmfeld der Organisation ermöglicht. Intern reknutieren im erweiterten Sinne bedeutet, daß Familienangehörige und Bekannte von in der Organisation Beschäftigten deutlich bessere Beschäftigungschancen haben als exteme Bewerber (Granovetter, 1988). Die Präferenz einer echten internen Allokation gilt ebenso für höhere Hierarchieebenen, zumal der exteme Arbeilsmarkt auf Grund der betriebsspezifischen Ausbildung und Erfahrungsmuster nahezu verschlossen bleibt. Das Verständnis von interner Mobilität schließt geringe interorganisationale Mobilität ein, d. h. konkret die Mobilität von Mitarbeitern von Regionalverwaltung zu Regionalverwaltung innerhalb der gleichen Einrichtung. Die interorganisationale Mobilität ist nicht Teil der Personalpolitik und kommt eher als Ausnahme vor.

Die exteme Personalwerbung ist auf ein Minimum beschränkt. Die Mitarbeiter verbleiben lange in der Organisation und entwickeln wenig Neigung, nach anderen Beschäftigungsmöglichkeiten Ausschau zu halten. Gute Bezahlung und Arbeitsbedingungen sind darauf gerichtet, die Mitarbeiter zu binden in Form eines langfristigen Beschäftigungsverhältnisses.

Nach den Kriterien, die Windolf/Hohn (1984) für die einzelnen Rekrutierungsstrategien entwickeln, korrespondiert die in dieser Organisation praktizierte Rekrutierungsstrategie mit der der Status-quo-Strategie. Hier ergibt sich zwischen Realität und Theorie ein hohes $\mathrm{MaB}$ an Übereinstimmung. Darüberhinaus ergeben sich Anhaltspunkte für eine empirische Bestätigung des im theoretischen Teil fortentwickelten Ansatzes von Windolf/Hohn in der Zuordnung von Organisationskonfiguration und Rekrutierungsstrategie. Das bedeutet, daß 
in einer Maschinenbürokratie in der Regel eine Status-quo-(Rekrutierungs-)Strategie anzutreffen ist.

\subsubsection{Funktionsketten innerhalb der Sozialversicherungsorganisation}

Die Funktionsketten in der Sozialversicherungseinrichrung sind auf Abteilungen bezogen und ermöglichen Übergänge zu anderen Abteilungen. Sie sind vertikal orientiert. Auch hier können nur exemplarisch zwei Funktionsketten im Vergleich vorgestellt werden.

Die unten abgebildete Funktionskette zeigt zwei Abteilungen innerhalb der Sozialversicherung, die auf der Basis des Funktionsniveaus miteinander verglichen werden. Hilfsweise wurde das Gehaltsgruppenniveau als Indikator für die Zuordnung zu einem Funktionsniveau hinzugezogen. Abteilungsspezifisch bestehen verschiedene Funktionsbezeichnungen. In der Abteilung 'Krankengesetz' ist die höchste Position (Stufe 6) besetzt. Alle höheren Funktionsniveaus sind nicht besetzt. Die Besetzungsstruktur der Abteilung ist so angelegt, daß die Hierarchie dieser Abteilung auf sechs Funktionsniveaus beschränkt ist. Die Abteilung 'Arbeitslosengesetz' zeigt Lücken in einer insgesamt stärker ausgeprägten

Abb. 18: Funktionsketten in der Sozialversichenung

\begin{tabular}{|c|c|c|}
\hline $\begin{array}{l}\text { Funktiongkette } \\
\text { 'operating core' } \\
\text { bzw. 'middle line' } \\
\text { "Rrankengesetz" }\end{array}$ & $\begin{array}{l}\text { Funktions- } \\
\text { niveau }\end{array}$ & $\begin{array}{l}\text { Funktionskette } \\
\text { 'operating core' } \\
\text { bzw. 'middle line' } \\
\text { "Arbeitslosengesetz" }\end{array}$ \\
\hline $\begin{array}{l}\text { Regintrationeange- } \\
\text { eteliter }\end{array}$ & 1 & Assistent \\
\hline $\begin{array}{l}\text { Verwaltungange- } \\
\text { ateliter }\end{array}$ & 2 & - \\
\hline Sachbearbeiter & 3 & Sachbearbelter \\
\hline Gruppenchef & 4 & Sachgebiet gbearbeiter \\
\hline $\begin{array}{l}\text { Gesetzentecbn. } \\
\text { Mitarbeiter }\end{array}$ & 5 & Sachgebiet sbearbeiter \\
\hline Abteilungoleiter & 6 & - \\
\hline - & 7 & Gruppenchef \\
\hline - & 8 & - \\
\hline - & 9 & Sachbereicholelter \\
\hline
\end{tabular}

Zeichenerklärung: -= Funktionsniveau ohne Besetzung

Hierichie, die über neun Funktionsniveaus verteilt ist. Die Lücken haben eine Beförderungsfunktion. Sie repräsentieren den 'Karrieresprung' zu einer anderen Funktion bzw. Gehaltsstufe. Eine andere Perspektive kommt in der Häufigkeit der Besetzung zum Ausdruck. Aufgrund des unterschiedlichen Arbeitsvolumens ist die Abteilung 'Arbeitslosengesetz' auf vergleichbaren Funktionsniveaus mit mehr Personen besetzt. Nach 
Stewman/Konda (1983) nehmen die in der Funktionskette zum Ausdruck kommenden Funktionsniveaus qua Personalbesetzung dann weniger die Form der pyramidenformigen Hierarchie als vielmehr die eines Flaschenhalses an mit unterschiedlicher 'Dicke' bzw. 'Breite' der Personalbesetzung. Ein solches Muster zeichnet sich tendenziell hier ab.

\subsection{Die Wirtschaftsprüfungsgesellschaft}

Das Wirtschaftsprüfungsunternehmen ist ein Dienstleistungsuntemehmen. Die Dienstleistung besteht aus der Anwendung von Wirtschafts- und Steuerrecht einschlieBlich des daraus resultierenden Beratungsbedarfs. Die Arbeit in Unternehmen dieser Art ist meistens sehr personenintensiv gestaltet. Aus dieser kurzen Beschreibung des Aufgabenfeldes ergibt sich, daß die damit verbundene Organisation professionelle Kennzeichen aufweist. So wie bei den bereils vorgestellten Organisationen soll auch hier geprüft werden, inwieweit die erhobenen Merkmale dem Organisationstyp entsprechen bzw. davon abweichen.

\subsection{Historische Entwicklung des Wirtschaftsprüfungsunternehmens}

Das Unternehmen wurde 1937 gegründet. Seit 1960 besteht das Untemehmen in der heutigen Form. Unternehmenszweck ist die Wirtschaftsprüfung mit allen Facetten, d. h. von der allgemeinen Rechnungslegung bis hin zur Steuerberatung. Die Mitarbeiter des Untemehmens verteilen sich auf mehrere Orte, an denen sich Zweigstellen des Unternehmens befinden. Seit 1986 besteht eine Fusion zwischen der Untemehmung und einer weltweit operierenden amerikanischen Wirtschaftsprüfungsgesellschaft. Beide Seiten bilden mit dieser Fusion neben einer strategischen Allianz (Douma/Schreuder, 1991) auch konkrete Kooperation auf den verschiedensten Ebenen.

\subsubsection{Branche, Aufgabenfeld, Markt und Organisationsumgebung}

Als professioneller Dienstleistungbetrieb gehört das Unternehmen mit insgesamt 165 Beschäftigten zu den Großen der Branche in der Region, jedoch im Rahmen der Untersuchung bildet das Unternehmen die kleinste Einheit von der Zahl der Beschäftigten her. Um den Hauptsitz des Unternehmens gruppieren sich sowohl innerhalb als auch außerhalb der Provinz vier Filialuntemehmen. Das Dienstleistungangebot ist umfassend. Die Kernaktivitäten liegen auf dem Gebiet der Wirtschaftsprüfung und der Rechnungslegung. Daneben werden Steuerberatung, buchungstechnische Dienstleistungen sowie Unternehmensberatung angeboten. Der Markt ist ein Insidemarkt, d. h. das Unternehmen baut vorwiegend informell (auf Empfehlung basierend) seinen Kundenkreis auf. Die positive Geschäftsentwicklung rechtfertigt eine permanente Expansion der Geschäftstätigkeit, was verbunden ist mit der Erweiterung des Personalbestandes.

\subsubsection{Aufbau der Wirtschaftsprüfungsorganisation}

An der Spitze des Untemehmens steht die Gesellschaftsversammlung, in der alle Gesellschafter und Partner die Unternehmensaktivitäten bestimmen. Als weitere Gremien auf der Leitungsebene finden sich der Vennotenrat (Beratungsorgan der Unternehmensinhaber) und der Vorstand, der für die täglich anfallenden Geschäfte verantwortlich ist. Verfolgt man die Hierarchie weiter nach unten, so wird eine Organisationsstruktur deutlich, die 
horizontal stark aufgegliedert ist. Eine Gesamtübersicht der Organisation ermöglicht das folgende Organigramm.

Abb. 19: Organigramm der Wirtschaftsprifungsgesellschaft

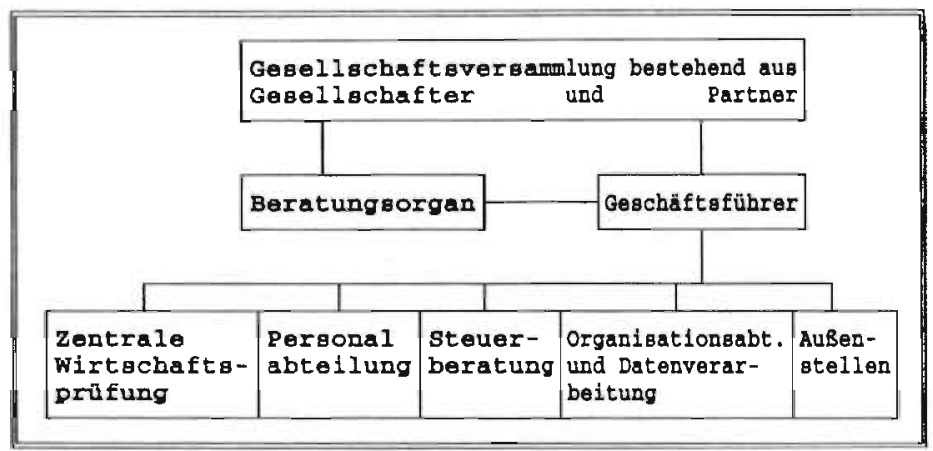

\subsubsection{Verteilung der Mitarbeiter auf Mintzbergs "Parts"}

Dem Part "strategic apex" lassen sich die Gesellschafter und Partner der Unternehmung zurechnen. Abteilungsleiter bzw, Leiter der Zweigstellen zählen zu den Personen, die die "middle line" prägen. Der Part "technostructure" setzt sich aus Mitarbeitem der Personalund der Kontrollabteilung sowie der Arbeitsautomatisierung zusammen. Den "support staff' bilden Schreibkrätte und Hilfspersonal. Als eine weitere Kategorie sind die Mitarbeiter zu nennen, die mit der eigentlichen Ausführung der Dienstleistung beschäftigt sind (operating core).

Die Wirtschaftsprüfungsgesellschaft verfügt über 165 Mitarbeiter. Diese verteilen sich über folgende Teile (parts) der Organisation: 
Abb. 20: Besetzung der Organisationsteile (parts) der Wirtschaftsprüfungsgesellschaft mit Beschäftigten zum Erhebungszeitpunkt 1989
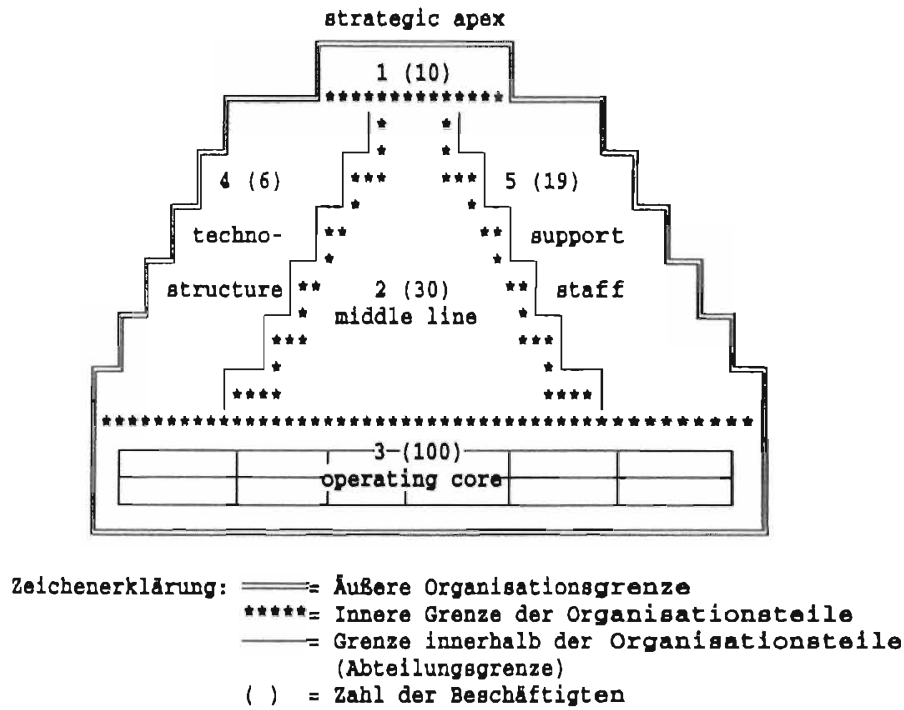

\subsubsection{Strategie und Perspektive der Wirtschaftsprüfungsgesellschaft}

Zur Strategie der Unternehmung gehört es, das behauptete Marktsegment zu verteidigen und mit kleinen Zuwachsraten die Geschäftstätigkeit weiter zu entwickeln. Durch Kooperation mit der amerikanischen Wirtschaftsprüfungsgesellschaft will man Zugang zum intemationalen Markt finden bzw. vom know how einer international operierenden Unternehmung profitieren (Soeters/Schreuder, 1988). Mitarbeitem der Wirtschaftsprüfungsgesellschaft wird die Gelegenheit geboten, für einen befristeten Zeitraum auf bisher weniger bekanntem Gebiet beim Kooperationspartner Erfahrung zu sammeln (Schreuder/ Soeters, 1986a; Soeters/Schreuder, 1986b).

Die wichtigsten Entscheidungen (u. a. auch strategische Entscheidungen) werden in der Gesellschafterversammlung getroffen, wobei die Partner, die als 'Unternehmensgründer' die Entscheidungen mittragen, einen stärkeren Einfluß ausüben.

Im Bereich der 'human resources' ist die Strategie des Unternehmens darauf gerichtet, qualifizierte Mitarbeiter zu werben bzw. Mitarbeiter anzuwerben, die bereit sind, innerhalb der Organisation über Lemerfahrungen professionelle Qualifikationen zu entwickeln.

\subsubsection{Merkmale der organisationsspezifischen Beschäftigungssituation}

Wie in den vorhergehenden Abschnitten gilt es, auch bei dieser Organisation, auf die verschiedenen Aspekte der Beschäftigungssituation einzugehen. 


\subsubsection{Zum Personalaufbau der Wirtschaftsprüfungsgesellschaft}

Der Lebensaltersaufbau der Beschäftigten dieser Organisation ist vergleichbar mit dem des Krankenhauses. Bis auf leichte prozentuale Abweichungen läßt sich ein ähnliches Strukturbild der Verteilung der Beschäftigten über alle Lebensaltersgruppen konstatieren. Gegenüber der Sozialversicherung handelt es sich bei der Wirtschaftsprüfungsgesellschaft um eine noch "junge" Organisation. Von der Dauer der Beschäftigung läßt sich ein vergleichbares Bild zu dem des Krankenhauses ableiten.

Die Geschlechterverteilung bringt zum Ausdruck, daß durchschnittlich zweidrittel der Beschäftigten Männer sind. In Bezug auf diesen Aspekt ist ein deutlicher Kontrast gegeben zur anderen professionellen Bürokratie, bei der die Geschlechterverteilung gradezu umgekehrt ist. In einem der folgenden Abschnitte wird auf der Basis eines Organisationsvergleiches dieser Aspekt weiter vertieft werden.

\subsubsection{Aus- und Weiterbildung sowie Beförderung}

Aus- und Weiterbildung genießen in der Wirtschaftsprüfungsgesellschaft einen hohen Stellenwert. Dies kommt zum Ausdruck in einem Weiterbildungsplan. Weiterbildung ist konzentriert auf Personen, die in der Praxis arbeiten und dort mit betriebswirtschaftlichen und fiskalen Problemen konfrontiert sind. Alle Abteilungen partizipieren an diesem Plan. Als Einführungskurse, die extern abgehalten werden, stehen der Computergebrauch sowie "Büropraxis" im Vordergrund. Weiterhin werden unter Leitung von erfahrenen Mitarbeitern intern Kurse gegeben. Ein Teil der Weiterbildungsaktivitäten wird extern vollzogen bzw. durch den Kooperationspartner gestaltet. Aus- und Weiterbildung stützt die Vorbereitung auf die Wirtschaftsprüferprüfung und fördert die professionelle Autonomie des Mitarbeiters.

\subsubsection{Personalphilosophie innerhalb des Wirtschaftsprüfungsuntemehmens}

Ein schriftlich fixiertes Personalführungskonzept besteht nicht. In einer Organisation, die von Professionals beherrscht wird, ist dies weniger erforderlich, da Arbeitnehmer über Autonomie (Benveniste, 1987) zur Ausfüllung ihrer Aufgabe verfügen. Dies bedeutet nicht, daß die Maßstäbe, die man sich hinsichtlich der Personalpolitik gesetzt hat, gering sind. Im Gegenteil, die Untemehmung baut auf die Qualifikation der Mitarbeiter. Das kommt zum Ausdruck in den schon erwähnten Weiterbildungsaktivitäten. Ebenfalls wird dies sichtbar in der Bereitschaft des Unternehmens, über einen relativ langen Zeitraum allgemein in Humankapital zu investieren, ohne daß dem kurzfristig ein 'retum on investment' gegenübersteht.

Die fundamentale Bedeutung der Personalpolitik kommt auch durch die Existenz einer Personalabteilung zum Ausdruck, die von vier Personen getragen wird. Der Stellenwert, den man der Personalführung einräumt, wird dabei deutlich. Die primäre Funktion ist neben Rekrutierung und Selektion von Personal die Begleitung in der Aus- bzw. Weiterbildungsphase. 


\subsubsection{Rekrutienung und Personalselektion}

Die Rekrutierung von Personal erfolgt auf verschiedenen Funktionsniveaus. Als 'klassisches Eintrittsniveau' für die Laufbahn in dieser Organisation gilt das Assistentenniveau, wobei wiederum verschiedene Eintrittsniveaus festgelegt sind. Man unterscheidet Assislenten, die minimal über einen mittleren Schulabschluß in kaufmännischer Richtung verfügen. Assistenten können ebenfalls mit Fach- bzw. Hochschulniveau eintreten. Das hier insgesamt gesehen höhere Ausbildungsniveau verkürzt tendenziell die Zeit der Ausbildung bis zur Prüfung zum Wirtschaftsprüfer.

Ein potentielles Rekrutierungsfeld ist die am Ort befindliche Universität, die im Rahmen des wirtschaftswissenschaftlichen Studiums eine Studienrichtung 'Accountancy' anbietet, die qua Ausbildungsinhalte den Wünschen der Untemehmung in Form der Qualifikationsstandards entgegen kommt. Intensive Kontakte zur Universität werden auf unterschiedlichen Kanälen unterhalten. Bei der Werbung von Assistenten mit geringerem Schulabschlußniveau geht man direkt zu Schulen, um potentielle Kandidaten "von der Schulbank weg" zu rekrutieren. 'Support staff' Personal wird vielfach von Arbeitnehmenüberlassungsuntemehmen rekrutiert.

Professionelles Personal wird, da der Markt knapp ist, von anderen Unternehmen abgeworben oder in einem spezifischen Umfeld angesprochen, beispielsweise in Finanzbehörden. Der Einstieg von Personal auf professioneller Ebene ist jederzeit möglich. Im Gegensatz zur Sozialversicherung wird bei der Wirtschaftsprüfungsgesellschaft von dieser Art "Seiteneinstieg" öfter Gebrauch gemacht. Dies läßı sich mit der stärker ausgeprägten interorganisationalen Mobilität und der Standardisierung der Qualifikation erklären.

Bei der Frage, welche Rekrutierungsstrategie nach Windolf/Hohn bei dieser Organisation praktiziert wird, gibt es keinen Zweifel. Bei der Wirtschaftsprüfungsgesellschaft dominiert die 'autonome Rekrutienungsstrategie'. Dies ergibt sich aus der Tatsache, daß Fachqualifikation deutlich im Vordergrund steht. Die Rekrutienangskanäle richten sich auf den Fachmarkt aber auch auf Bildungseinrichtungen, in denen ausbildungswillige potentielle Kandidaten anzutreffen sind. Auch bei dieser Organisation zeigt sich in bescheidenem Umfang die Inanspruchnahme der flexiblen Strategie, durch Rekrutierung von Personal im 'support staff'-Bereich von Arbeitnehmerüberlassungsuntemehmen (uitzendbureau).

Bei dieser Organisation wird die theoretisch konstatierte Verbindung zwischen Organisationskonfiguration und Rekrutierungsstrategie bestätigt. Mit der professionellen Bürokratie läßt sich demnach die 'autonome Rekrutierungsstrategie' assoziieren und tatsächlich antreffen.

\subsubsection{Funktionsketten innerhalb des Wirtschaftsprüfungsuntemehmens}

Innerhalb der Wirtschaftsprifungsgesellschaft besteht eine für die potentielle Mobilität vorgezeichnete Funktionskette. Die formellen Funktionsniveaus sind im Kontext anderer Aufgabenstellungen stets dieselben (Dillard/Ferris, 1989). 
Abb. 21: Funktionskette in der Wirtschaftsprüfungsgesellschaft, vom 'operating core' über 'middle line' zum 'strategic apex'

Funktionskette

"Wirtbchafteprüfung"

Agsiotent

Berufsassistent

Wirtschaftoprüfer

Partner

Gesellochafter

\begin{tabular}{|c|}
\begin{tabular}{l} 
Funktions- \\
niveau \\
1 \\
\hline 2 \\
\hline 3 \\
\hline 4 \\
\hline 5
\end{tabular} \\
\hline
\end{tabular}

Diese Funktionskette ist die vorgezeichnete mögliche Laufbahn des Wirtschaftsprüfers. Sie verläuft über 'operating core', 'middle line' zum 'strategic apex'. Im Gegensatz zu anderen Organisationen erstreckt sich diese Funktionskette über die gesamte vertikale Länge der Organisation. Der kritische Punkt ist der Übergang von Funktionsniveau 2 nach Funktionsniveau 3. Mobilitätshindernis ist die Prifung zum Wirtschaftsprifer. Viele Assistenten (auch Berufsassisten) haben in ihrem beruflichen Werdegang diese Funktionshürde vor Augen. Viele verbleiben auf dem Funktionsniveau des Berufsassistenten, weil es ihnen nicht gelingt, diese Hürde zu nehmen. Erfolgreichen Kandidaten steht der weiteren Mobilität auch interorganisational nichts im Wege. Der Zugang zur Organisation ist auf allen Hierarchieniveaus möglich. Damit unterscheidet sich diese Organisation von dem nach außen geschlossenen Strukturmerkmal interner Arbeitsmärkte.

\subsection{Das Produktionsunternehmen}

Das Produktionsunternehmen repräsentiert ein Untemehmen, dessen Aktivität auf die Gewinnung und Verarbeitung und Vermarktung von Rohstoffen zielt.

\subsubsection{Historische Entwicklung des Produktionsuntemehmens}

Das Produktionsunternehmen wurde 1926 gegründet in einer Region, in der Kalkstein in ökonomisch abbaubaren Mengen vorzufinden ist. Das Hauptstandbein der Untemehmung in der Vergangenheit bis heute war die Produktion von Zement.

Dic Entwicklung des Produktionsuntemehmens innerhalb der letzten Jahre ist durch folgende Schwerpunkte gekennzeichnet: Der Produktabsatz hat in den Jahren 1970-1980 stetig abgenommen. 1984 wurde der Tiefpunkt im Absatz erreicht. Zunächst war die Strategie daraut gerichtet, so viel wie möglich selbst 'in die Hände zu nehmen' (Maximalisierung interner Beschäftigung). Nun ist die Unternehmensleitung von dieser Zielsetzung abgekommen. Das iußert sich in Form der Einführung der Vomuhestandsregelung und der Optimalisierung von Beschäftigung bei den Hauptaufgaben. Jetzt werden Dienste (Bewachung, Kantine, baukundiger Unterhalt sowie Engineering) ausgelagert, d. h. sie werden von externen Untemehmen ausgeführt. Dies ermöglicht mehr Konzentration auf den eigentlichen Leistungbereich und erlaubt zudem ein transaktionskostengünstigeres Resultat. 
Es entspricht der an anderer Stelle geäuBerten Strategie des sich Besinnens auf die Hauptaufgaben, während typische "Support-Funktionen" ausgelagert werden.

\subsubsection{Branche, Aufgabenfeld, Markt und Organisationsumgebung}

Das Produktionsuntemehmen ist Teil einer Untemehmensgruppe, die sich historisch entwickelt hat aus der ursprünglichen Zementrohstoffgewinnung und deren Verarbeitung. Die Untemehmensgruppe beinhaltet folgende Untemehmensaktivitäten: die Gewinnung der Rohstoffe und die Verarbeitung zu verschiedenen Sorten Zement. Eine weitere Unternehmensaktivität ist konzentriert auf die Fertigung von Betonmörtel. Eine Reihe von Tochteruntemehmungen produzieren Betonwaren, Straßenbaumaterialien sowie Düngemittel und die Dienstleistung "Transport". Ein Tochteruntemehmen ist spezialisiert auf die Produktion von Hochofenzement. Das Untemehmen befindet sich an einem anderen Standort. Der Absatz der Produkte findet hauptsächlich in den Niederlanden statt. Ein Teil der Produkte wird exportiert. Marketing und Absatz erfolgen überwiegend durch das Verkaufsbüro, das im Zentrum der Niederlande etabliert ist. Die Auslandsaktivitäten erstrecken sich auf ein Verkaufsbüro in Deulschland und eine Produktionsstätte in Belgien. Hier werden Klinker-Baumaterialien gefertigt. Insgesamt verfügt die Untemehmensgruppe über ein Beschäftigungsvolumen von rund 1300 Arbeitnehmern.

\subsubsection{Aufbau des Produktionsuntemehmens}

Die Organisation des Produktionsuntemehmens ist sowohl horizontal als auch vertikal stark differenziert bzw. dezentralisiert. Der Direktion unterstehen alle Hauptabteilungen. Von den in der Abbildung erfaßten Geschäftsbereichen gehen weitere Aufteilungen in Unterabteilungen aus. 
Abb. 22: Organigramm des Produktionsbetriebes

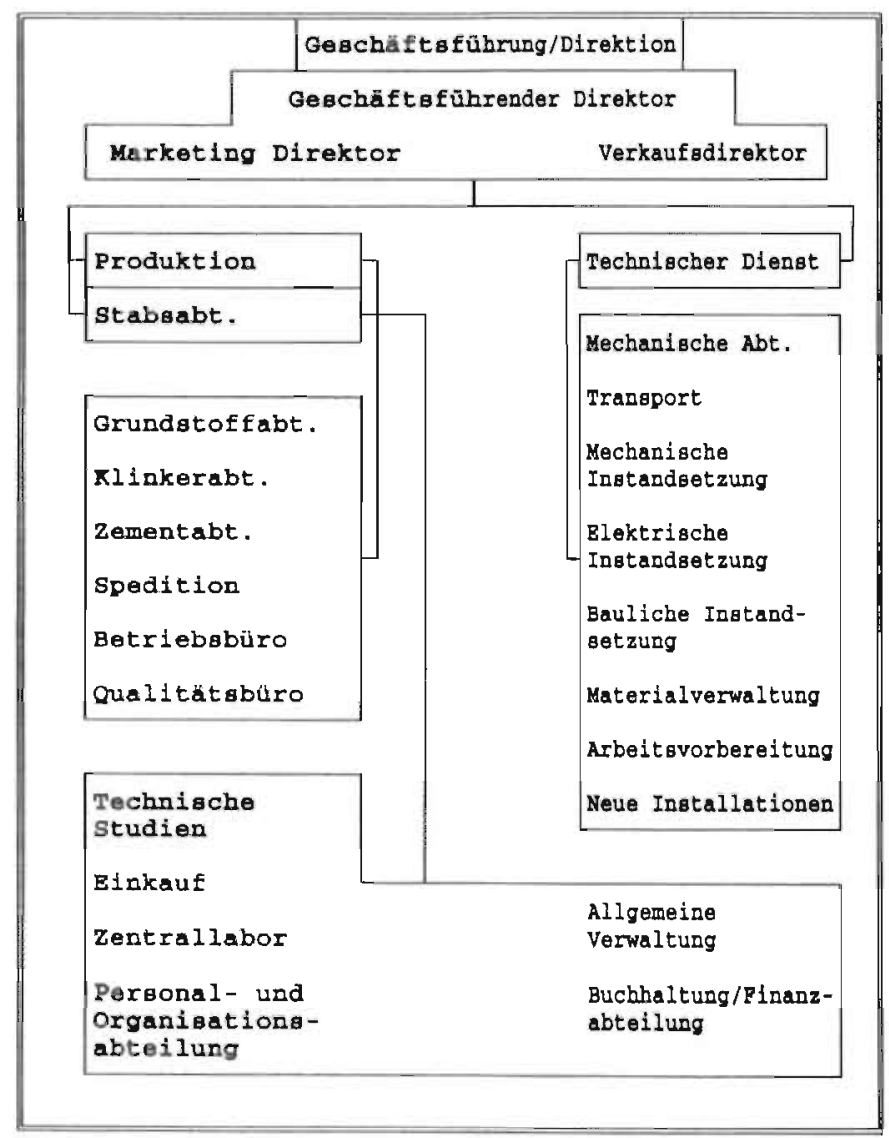

\subsubsection{Strategie und Perspektive des Produktionsunternehmens}

Dieser Abschnitt ist konzentriert auf die langfristige Entwicklung des Produktionsunternehmens. Er behandelt die wichtigsten strategischen Probleme, die strategische Entscheidungsfindung sowie Probleme, die Bezug nehmen auf Human Resource Management.

In der Reihenfolge ihrer Bedeutung sind folgende strategische Probleme zu nennen: Die Entwicklung des Baumarktes im Rahmen der europäischen Integration mit Datum 1992 ist für den Absatz der Produkte von großer Bedeutung. Von dieser Entwicklung hängt entscheidend die weitere Entwicklung und auch die Behauptung gegenüber der Konkurrenz ab. Die EntwickIung wird tangiert durch die Importe aus Süd- und Osteuropa, 
die noch beschränkt sind durch bürokratische Restriktionen. Außerdem wird die zukünftige Entwicklung getragen durch die Entwicklung des inlándischen Marktes.

Darüberhinaus ist die Entwicklung der Umweltproblematik von Bedeutung, da das Unternehmen durch Rohstoffabbau im Tagebau landschaftsverändernde Effekte bewirkt. Aufgrund des gestiegenen Umweltbewußtseins können daraus Restriktionen für das Unternehmen resultieren. Da eine weitere Rohstofferschließung als Folge der Umweltproblematik nicht ermöglicht wird, könnte langfristig ein Defizit an Kieselrohstoff auftreten.

Die strategische Entscheidungsfindung ist gekennzeichnet durch eine genaue Umschreibung der Zielvorstellungen des Managements. Es besteht ein strategischer Plan, der für die Dauer von einem Jahr aufgestellt wird. Daneben existiert ein Mehrjahresplan, der über drei Jahre gültig ist, wobei das erste Jahr das Planjahr mit Budget ist. Informationsquellen, über die strategische Informationen aus der Organisationsumgebung kommen, sind meistens untermehmensextern anzusiedeln. Sie sind nach Auskunft der Entscheidungsträger eindeutig und gehen zu 66 Prozent auf persönliche Quellen der Unternehmensleitung zurïck. Die Verantwortlichkeit für die Entwicklung und Formulierung strategischer Untemehmensführung und die daraus abgeleitete Personalführung trägt die Direktion des Produktionsuntemehmens.

In Bezug auf human resources sind die wichtigsten Probleme folgendermaßen zu charaklerisieren:

-Angestrebt wird eine Produktivitätssteigerung, die vor allem erreicht werden soll durch Modernisierung der Produktion und neue Investitionen bei Maschinen.

-Eine solche Zielrealisienung bedeutet ein Wegfall von Arbeitsplätzen. Darauf hat man sich im Produktionsuntemehmen in Form von Umschulung der Mitarbeiter eingestellt. Ausund Weiterbildung von Mitarbeitern kommt in diesem Zusammenhang einem "Anpassungsprogramm" nahe.

-Ein weiteres langfristiges human-resource-Problem besteht in der "Vergreisung" des Personals. Das Durchschnittsalter der Beschäftigten liegt bei 45 Jahren. Die Möglichkeit, über die Vomuhestandsregelung (VUT) die betriebliche demographische Entwicklung zu beeinflussen, wird als unzureichend angesehen. Obwohl in den abgelaufenen Jahren die Inanspruchnahme der Vorruhestandsregelung groß war, hat dies noch nicht zu einer deutlichen Veränderung der demographischen Entwicklung des Unternehmens geführt.

-Die Beschäftigungssitation gibt nach Auffassung der Geschäftsleitung in der nahen Zukunft AnlaB zur Sorge. Ein Beschäftigungsabbau ist wahrscheinlich. Für die Unternehmensleitung zeichnet sich die Tendenz $a b, d a b$ mehr Produktivität zu geringerem Beschäftigungsniveau führt.

\subsubsection{Verteilung der Mitarbeiter auf Mintzbergs "Parts"}

Die Unternehmensleitung (strategic apex) besteht aus drei Personen. "Middle line" umfaßt das Abteilungsleiterniveau. Die "technostructure" wird von den im Organigramm genannt- 
en Stabsstellen geprägt. Auf Grund der vielfäligen Dienstleistungen insbesondere in technischen Bereich erscheint der Part "support staff" als groß. Hierzu rechnen Handwerker und Unterhaltspersonal. Mit der eigentlichen Produktion befaßt sind die Mitarbeiter des Parts "operating core."

Das Produktionsunternehmen hat 820 Mitarbeiter. Diese verteilen sich über folgende Teile (parts) der Organisation :

Abb. 23: Besetzung der Organisationsteile (parts) des Produktionsuntemehmens mit Beschäftigten zum Erhebungszeitpunkt 1989
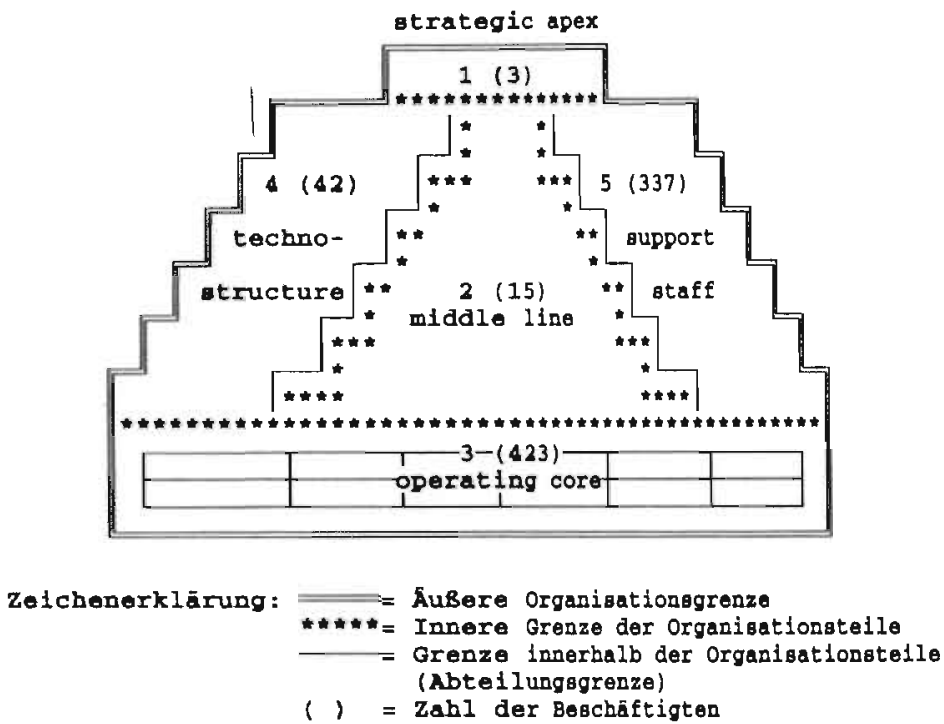

Bei dieser Organisation fällt die starke Besetzung des 'support staffs' auf, eine Erscheinung, die mit der des Krankenhauses qua Ausprägung vergleichbar ist.

\subsubsection{Merkmale der organisationsspezifischen Beschäftigungssituation}

Auch bei dieser Organisation soll neben den bereits genannten Faktoren auf weitere Einzelheiten der Beschäftigungssituation eingegangen werden.

\subsubsection{Zum Personalaufbau des Produktionsuntemehmens}

Im Vergleich zu den bereits vorgestellten Organisationen repräsentiert das Produktionsuntemehmen die "älteste" Organisation was den Personalaufbau betrifft. Wie schon angedeutet liegt das Durchschnitcalter bei 45 Jahren. Die Organisation gilt damit als deutlich "überaltert". Diese Erkenntnis schlägt sich auch nieder in der Dauer der Tätigkeit 
der Beschiaftigten. Nahezu ein Drittel der Belegschaft ist zwischen 20 bis 30 Jahren im Untemehmen tätig.

Bei der Betrachtung der Geschlechterverteilung fällt auf, daß es sich um eine beinahe ausschließlich "maskuline" Organisation handelt. Frauen sind zu einem Anteil von 7 Prozent in der Organisation anwesend. Der geringe Frauenanteil ist zu erklären aus der früher schweren Tätigkeit in Produktion und Verarbeitung, die eigentlich nur Männem zugemutet werden konnte. Im "support staff" finden sich relativ mehr Frauen.

\subsubsection{Aus- und Weiterbildung sowie Beförderung im Produktionsuntemehmen}

Weiterbildung und Beförderung stehen im Produktionsunternehmen in einem ursächlichen Zusammenhang. Aus- und Weiterbildungsaktivitäten bilden die Voraussetzung für Beförderungen. Das Spektrum der Aus- und Weiterbildungsaktivitäten reicht von der internen Managementschulung bis hin zu externen Kursen. Finanziell wird von Seiten der Unternehmensleitung das Interesse an besserer Qualifikation unterstiitzt. Aus- und Weiterbildung variiert von niedrigem bis $z u$ hohem Erfahrungsniveau und über alle Abteilungen.

Im Jahr 1988 haben 174 Arbeitnehmer an einer oder mehreren internen Weiterbildungsmaßnahmen teilgenommen. Daneben wurden Kurse organisiert im Bereich "Sicherheitsvorschriften", Qualitätsüberwachung, Computersimulation und "Sauerstoffinstallation". Rund hundert Mitarbeiter haben im Jahr 1988 an einer extemen Ausbildung teilgenommen. Im Vordergrund standen dabei technische Fächer sowie Automatisierung. Selbst Sprachkurse und Computerkurse finden bei den Mitarbeitern Resonanz, was die Teilnahme von 78 Personen bestätigt.

Außerdem besteht ein intemes Umschulungsprogramm, das eine Konsequenz darstellt aus der Strategie "zurück zu den Kernaufgaben" (back to the basics). So werden Dienstleistungen, beispielsweise Bewachung u. a. durch exteme Unternehmen wahrgenommen. Diese Umschulung erstreckt sich auf eine Laufzeit von drei bis vier Jahren und betrifft rund siebzig Mitarbeiter.

Das ständige Bedürfnis der Organisation, Mitarbeiter besser zu qualifizieren, kommt zum Ausdruck in der Organisations- und Funktionsstruktur, die darauf gerichtet ist, Mitarbeiter zu fördem.

Die Beförderungsmöglichkeiten jedoch sind beschränkt. Ein Beförderungsautomatismus besteht nicht. Die Kriterien, nach denen eine Beförderung erfolgt, setzen sich zusammen aus einer Kombination der Kriterien: Leistung, Qualifikationsverbesserung und Seniorität. Die Entwicklung, die Personen in der Organisation durchlaufen, wird durch feste Funktionsmuster bestimmt, die meistens in Form von Funktionsketten angeordnet sind.

\subsubsection{Personalphilosophie innerhalb des Produktionsuntemehmens}

Beim Produktionsuntemehmen ist die Personalphilosophie schriftlich als "soziale Richtlinie" (sociaalbeleid) formuliert. Die "sozialen Führungsgrundsäıze" gelten als wesentlicher 
und untrennbarer Teil der Führungsgrundsätze der ganzen Unternehmung. Die "sozialen Führungsgrundsätze" lassen sich auf folgende Punkte konzentrieren:

1 die Entwicklung einer flexiblen und effektiven Organisation, wodurch die Untemehmensziele realisiert werden können und die Mitarbeiter ihren Arbeitsbeitrag als sinnvoll erfahren können;

2 im Rahmen der Kontinuität die Gewährleistung von Beschäftigung und die Bereitstellung von Personal, um heutige und zukünftige Aufgaben gut und effizient ausführen zu können;

3 ein umfassendes Paket Arbeitsbedingungen, die Einsatz und Kreativität der Mitarbeiter stimulieren und honorieren und den AnschluB an die gesellschaftliche Entwicklung gewährleisten;

4 die Handhabung guter Arbeitsbedingungen und eines guten Arbeitsklimas.

Die Praktizierung dieser "Führungsgrundsätze" bezieht sich nicht allein auf die Unternehmensleitung sondern auf alle Leitungsebenen des Unternehmens. Jeder Mitarbeiter erfährt bei Organisationseintritt von den Zielsetzungen des Untemehmens in Form einer "Zielrichtlinie" (beginselverklaring). Mit der Aushändigung wird erwartet, daß jeder Mitarbeiter davon Kenntnis nimmt und diese auch im Arbeitsverhalten versucht umzusetzen.

Es entspricht einer dezentralen innerbetrieblichen Personal- und Organisationspolitik, daß Entscheidungskompetenzen möglichst auf niedrigstem Niveau anzusiedeln sind und daß die Tragweite der Entscheidung zu überschauen ist. Ein Informationssystem unterstützl die Tendenz zur dezentralen Entscheidung.

Neben der allgemeinen Sicherung und Schaffung neuer Arbeitspläıze steht die Förderung Jugendlicher in Arbeitserfahrungsprojekten, die Anwendung von Teilzeitarbeil, Karriereförderung sowie Lemarbeitsverträge zentral.

\subsubsection{Rekrutierung und Personalselektion im Produktionsuntemehmen}

Die Rekrutierungspolitik ist darauf gerichtet, Mitarbeiter anzuwerben und zum Bleiben in der Organisation zu ermutigen. Im Vordergrund stehen dabei gut ausgebildete Mitarbeiter, die auch für zukünftige Aufgaben eingesetzt werden können. Bei der Besetzung einer offenen Stelle wird zunächst betriebsintem die Stelle ausgeschrieben. Damit wird im Rahmen von Personalentwicklung Interesse daran bekundet, geeigneten Mitarbeitem die Gelegenheit zu geben, sich intem zu bewerben. Erst nachdem feststeht, daß intem keine geeigneten Mitarbeiter für die Besetzung der offenen Stelle zur Verfügung stehen, geht man über zur Werbung und Rekrutierung auf dem extemen Arbeitsmarkt.

Bei der Personalplanung genießen Mitarbeiter, die sich in Ausbildung befinden sowie behinderte Mitarbeiter Priorität, was in der Reservierung von Arbeitsplătzen für diese Gruppen zum Ausdruck kommt. 
Neue Mitarbeiter werden in der Regel für eine Funktion rekrutiert. Dabei werden die weiteren Entwicklungsmöglichkeiten von Arbeitgeberseite als auch die Wünsche der Arbeitnehmer bedacht.

Das Prinzip des "internen Arbeitsmarktes" gilt bis zum HBO-Niveau (Fachhochschulniveau). Dies kortespondiert mit dem Mittelmanagementfunktionsniveau. Höhere Funktionen werden nicht immer besetzt mit Personen, die innerbetriebliche Erfahrung gewonnen haben. Hier wird unter Berücksichtigung der Priorität des internen Arbeitsmarktes (erst intern vor extern werben) von der externen Rekrutierungsmöglichkeit Gebrauch gemacht.

Im übrigen gilt bei der Selektion von Personal ein Selektionscode der Niederländischen Personalvereinigung, der eine Art "fair play" Behandlung der betroffenen Person im Selektionsverfahren gewährleisten soll.

Beim Produktionsunternehmen existiert nicht nur eine Rekrutierungsstrategie. Vielmehr geht es um zwei Reknutienungsstrategien, die nach der von Windolf/Hohn entwickelten Typologie hier zutreffen. Zum einen handelt es sich um die Status-quo-Strategie, die insbesondere ein Netzwerk unterstelt und idealtypisch zutrifft für den internen Arbeitsmarkt. Das Netzwerk besteht aus Familienangehörigen und Personen, die im weitesten Sinne dem erweiterten internen Arbeitsmarkt zugerechnet werden können. Neue Mitarbeiter betreten die 'ports of entry' mit dem Ziel einer Ausbildung. Nach Vollendung der Ausbildung zeichnen sich dann 'Karrierepfade' ab, deren Beschreiten dann ermöglicht wird. Insbesondere in der Vergangenheit dominierte diese Rekrutierungsstrategie. Sie wird zunehmend flankiert von der 'autonomen Rekntierungsstrategie'. Der Bedarf an Fachpersonal insbesondere mit technischem Ausbildungshintergrund läßt dieser Rekrutierungsstrategie mehr Bedeutung zukommen.

Die Zuordnung von Organisationskonfigurationen zu den Rekrutierungsstrategien legt auch hier eine differenziertere Betrachtung nahe. Die Status-quo-Strategie spricht für die Maschinenbürokratie, während die autonome Strategie mit der professionellen Bürokratie weitgehend im Einklang steht. Die quantitative Untersuchung wird hier mehr Klarheit schaffen und aufzeigen, welche Rekrutierungsstrategien im Kontext der einen oder anderen Organisationskonfiguration oder als Mischform hier zutreffen.

\subsubsection{Funktionsketten im Produktionsuntemehmen}

Von den zahlreichen unterschiedlichen Funktionsketten sollen hier zwei gegenübergestellt werden. Auch hier wird eine "lange" mit einer "kurzen" Funktionskette exemplarisch verglichen. Die Funktionsketten sind aufgrund der Aufgabeninhalte recht heterogen und dadurch auch schwierig zu vergleichen. Wir sprechen hier eine Funktionskette an, die im Bereich des 'operating core' des Untemehmens anzusiedeln ist. 
Abb. 24: Funktionsketten im Produktionsunternehmen (operating core)

\begin{tabular}{|c|c|c|}
\hline $\begin{array}{l}\text { Punktionsketto } \\
\text { "Grundetoffe" }\end{array}$ & $\begin{array}{l}\text { Punktions- } \\
\text { niveau }\end{array}$ & $\begin{array}{l}\text { Funktiongkette } \\
\text { "Produktion" }\end{array}$ \\
\hline $\begin{array}{l}\text { Binarbeitender } \\
\text { Maschinlot }\end{array}$ & 1 & - \\
\hline Maschinist & 2 & $\begin{array}{l}\text { Bintretender } \\
\text { Bediener }\end{array}$ \\
\hline Maschinist Grube & 3 & Bediener \\
\hline 1. Maschinist & 4 & Operator \\
\hline Vorarbeiter & 5 & - \\
\hline
\end{tabular}

Das Funktionsniveau entspricht auch hier den Gehaltsstufen. Während bei der Funktionskette "Grundstoffe" eine durchgehende Abfolge von Funktionen besteht, beginnt die Funktionskette "Produktion" erst bei Funktionsniveau 2 und endet auf Niveau 4.

Die Vielfältigkeit der Funktionsketten insbesondere in den Maschinenbürokratien erfordert eine systematische Durchdringung und Beurteilung nach den verschiedensten Kriterien. Dem Organisationsvergleich der Organisationen auf der Basis von Funktionsketten sind damit deutliche Grenzen gesetzt.

\subsection{Organisationsvergleich von Arbeitsmarktcharakteristiken}

Dieser Abschnitt behandelt die quantitativen und qualitativen Charakteristiken der betrieblichen Beschäftigung. Das Zahlenmaterial wird hier in organisationsvergleichender Weise präsentiert. Die Informationen stammen aus der qualitativen Erhebung.

\subsubsection{Demographischer Aufbau der Belegschaft der Organisationen}

In der folgenden Tabelle kommt die Lebensaltersverteilung über einen Zeitraum von vier Jahren zum Ausdruck. Das Zahlenmaterial weist das Krankenhaus und die Wirtschaftsprüfungsgesellschaft als relative "junge Organisationen" auf der Basis des Lebensalters der Beschäftigten aus im Gegensatz zur Sozialversichenung und zum Produktionsuntemehmen. Bei letzteren ist die erste Lebensalterskategorie (jünger als 20) ausgesprochen schwach bzw. überhaupt nicht besetzt. Die Berechnung basiert auf Informationen aus dem Personalinformationssystem der jeweiligen Organisation. 
Tab. 6: Lebensaltersverteilung im Zeitraum 1984, 1986, 1988

in vier Organisationen in Prozenten

\begin{tabular}{|c|c|c|c|c|}
\hline \multicolumn{2}{|c|}{ Organisation } & \multirow{3}{*}{$\begin{array}{l}S \\
84-88\end{array}$} & \multirow{3}{*}{$\begin{array}{l}W \\
84-88\end{array}$} & \multirow{3}{*}{\begin{tabular}{|l}
$\mathrm{P}$ \\
$84-88$
\end{tabular}} \\
\hline & $\mathbf{R}$ & & & \\
\hline Lebeng & $84-88$ & & & \\
\hline & $\%$ & $\%$ & $\%$ & $\%$ \\
\hline als 20 & 2.5 & 0.6 & 14.3 & 0 \\
\hline $20-29$ & 50.7 & 33.4 & 38.2 & 11.7 \\
\hline $30-39$ & 29.7 & 35.6 & 28.7 & 24.2 \\
\hline $40-49$ & 11.3 & 17.6 & 8.1 & 37.9 \\
\hline $50-59$ & 5.2 & 12.3 & 9.4 & 25.7 \\
\hline $\begin{array}{l}60 \text { und } \\
\text { gilter }\end{array}$ & 0.6 & 0.5 & 1.3 & 0.5 \\
\hline & 10 & .00 & 100 & 100 \\
\hline
\end{tabular}

$\mathrm{K}=$ Krankenhaus; $\mathrm{S}=$ Sozialversicherung; W=Wirtschaftsprüfungsunternehmen; $\mathrm{P}=$ Produktionsunternehmen

Als Schußfolgerung bleibt festzuhalten, daß Parallelen im demographischen Aufbau zwischen professionellen Bürokratien, repräsentiert durch Organisationen wie Krankenhaus und Wirtschaftsprüfungsgesellschaft und Organisationen der Maschinenbürokratie, repräsentiert durch Sozialversicherung und Produktionsunternehmen, gegeben sind. Während professionelle Bürokatien im Rahmen der hier einbezogenen Organisation als 'junge Organisationen' angesehen werden können, zeichnet sich bei den anderen Organisationen ein deutlich höheres 'Altersniveau' ab.

6.5.2 Das Verhältnis Männer/Frauen 1984, 1986, 1988

in den untersuchten Organisationen

Das Verhältnis von Männern und Frauen in den untersuchten Organisationen ermöglicht Einsicht in die Personalstruktur. Bei der folgenden Interpretation des Zahlenmaterials sollen einerseits das Geschlechterverhältnis im Vordergrund stehen, andererseits die Verschiebungen im Zeitablauf.

Zur Charakterisierung der Geschlechterverteilung innerhalb der Organisationen ergibt sich folgendes Bild. Die folgende Tabelle liegt dieser Betrachtung zugrunde.

Das Krankenhaus stellt deutlich eine 'feminine' Organisation dar, basierend auf einen Frauenanteil von rund 74 Prozent. Im Gegensatz dazu ist das Produktionsunternehmen eine ausgesprochen 'maskuline' Organisation mit 93 Prozent Männem. Bei der Wirtschaftsprüfungsgesellschaft entspricht das Verhältnis Mann-Frau einem Verhältnis von rund zweidrittel zu eindrittel. Die Sozialversicherung hat von allen Organisationen die ausgewogenste Geschlechterverteilung. Rund 55 Prozent Männer stehen 45 Prozent Frauen gegenüber. 
Tab. 7: Verteilung Männer/Frauen im Jahre 84, 86, 88 in Prozenten

\begin{tabular}{|c|c|c|c|c|c|c|c|c|c|c|c|c|}
\hline \multirow[b]{2}{*}{ t } & \multicolumn{3}{|c|}{ Organisation } & & & \\
\hline & $\begin{array}{l}\pi \\
84\end{array}$ & ${ }^{86}$ & ${ }^{88}$ & $\begin{array}{l}5 \\
84 \\
{ }_{3}\end{array}$ & ${ }^{86}{ }_{\%}$ & 88 & $\begin{array}{l}\text { W } \\
84\end{array}$ & ${ }^{86}$ & ${ }_{8}^{88}$ & $\begin{array}{l}P \\
84 \\
{ }_{q}\end{array}$ & ${ }^{86}{ }_{\%_{6}}$ & ${ }^{88}{ }_{9 / 6}$ \\
\hline Mánerer & 26 & 26 & 27 & 58 & 55 & 54 & 69 & 72 & 67 & 93 & 93 & 93 \\
\hline & 74 & 74 & 73 & 42 & 45 & 46 & 31 & 28 & 33 & 7 & 7 & 7 \\
\hline & 100 & 100 & 100 & 100 & 100 & 100 & 100 & 100 & 100 & 100 & 100 & 100 \\
\hline
\end{tabular}

Zeichenerklärung: $\mathrm{K}=\mathrm{K}$ rankenhaus; $\mathrm{S}=$ Sozialversicherung;

$\mathrm{W}=$ Wirtschaftsprüfungsunternehmen; $\mathrm{P}=$ Produktionsuntemehmen

In Bezug auf die jeweiligen Organisationskonfigurationen läßt sich kein besonderes Muster ableiten. Dies gilt auch für die Betrachtung der Entwicklung von 1984 bis 1988. Während sich im Zeitablauf für die Organisationen Krankenhaus, Sozialversicherung und Wirtschaftsprüfungsgesellschaft leichte prozentuale Veränderungen ergeben, bleibt die Geschlechterverteilung im Zeitablauf beim Produktionsunternehmen konstant.

\subsubsection{Durchschnittliche Tätigkeitsdauer von Arbeitnehmern per Jahr}

Ein Merkmal des intemen Arbeitsmarktes ist die langfristige Beschäftigungsdauer. In diesem Abschnitt geht es darum, festzustellen, wieviel Beschäftigte über welchen Zeitraum welcher Organisation angehören. Im Prinzip werden die Aussagen zur demographischen Entwicklung hier über die Dauer der Beschäftigung bestätigt. Den folgenden Überlegungen liegt diese Tabelle zugrunde:

Tab. 8: Durchschnittliche Tätigkeitsdauer von Arbeitnehmem (für die Jahre 1984, 1986, 1988) in Prozenten

\begin{tabular}{|c|c|c|c|c|c|c|c|c|c|c|c|c|}
\hline $\begin{array}{l}\text { Jahr } \\
\text { Dauer }\end{array}$ & $\begin{array}{l}\text { Oro } \\
\text { K } \\
\mathbf{8 4} \\
\text { \% }\end{array}$ & $\begin{array}{r}\text { nise } \\
86 \\
\%\end{array}$ & $\begin{array}{l}\text { Ion } \\
88 \\
\%\end{array}$ & $\mid \begin{array}{l}5 \\
84 \\
\%\end{array}$ & $\begin{array}{r}86 \\
\%\end{array}$ & $\begin{array}{r}88 \\
\%\end{array}$ & $\mid \begin{array}{l}W \\
84 \\
\%\end{array}$ & $\begin{array}{l}\text { B6 } \\
\\
\$ 6\end{array}$ & $\begin{array}{r}88 \\
\%\end{array}$ & \begin{tabular}{|l}
$\mathbf{P}$ \\
84 \\
\\
$\%$
\end{tabular} & $\begin{array}{l}86 \\
\%\end{array}$ & $\begin{array}{l}88 \\
8\end{array}$ \\
\hline$<1$ & 8 & 9 & 6 & 1 & 12 & 6 & 13 & 22 & 29 & 1 & 0 & 1 \\
\hline $1-2$ & 6 & 6 & 8 & 1 & 4 & 8 & 6 & 12 & 18 & 2 & 1 & 3 \\
\hline $2-5$ & 31 & 19 & 21 & 23 & 7 & 14 & 31 & 16 & 18 & 7 & 6 & 5 \\
\hline $5-10$ & 29 & 34 & 30 & 34 & 29 & 21 & 19 & 24 & 14 & 11 & 15 & 13 \\
\hline $10-20$ & 21 & 27 & 29 & 35 & 41 & 38 & 22 & 17 & 12 & 38 & 31 & 32 \\
\hline $20-30$ & 4 & 4 & 5 & 2 & 2 & 9 & 9 & 9 & 9 & 29 & 34 & 33 \\
\hline$>30$ & 1 & 1 & 1 & 4 & 5 & 4 & 0 & 0 & 0 & 12 & 14 & 13 \\
\hline total & 100 & 100 & 100 & 100 & 100 & 100 & 100 & 100 & 100 & 100 & 100 & 100 \\
\hline
\end{tabular}

Zeichenerklarung: $\mathrm{K}=\mathrm{Krankenhaus;} \mathrm{S}=$ Sozialversicherung; $\mathrm{W}=$ Wirtschaftsprüfungsuntemehmen; $\mathrm{P}=$ Produktionsuntemehmen 
Lang andauemde Beschäftigungsverhältnisse mit einer Beschäftigungsdauer von 10 bis 20 Jahren sind in jeder Organisation vorhanden. Die starke Besetzung allein dieser Kategorie der Beschäftigungsdauer variiert von Organisation zu Organisation. Dennoch gibt es auch hier wieder - orientiert an der Organisationstypologie - Unterschiede. Krankenhaus und Wirtschaftsprüfungsgesellschaft als professionelle Bürokratien zeigen für die Beschäftigungsdauer deutlich geringere Besetzungzahlen als Maschinenbürokratien. So liegt der Prozentsatz für Krankenhaus und Wirtschaftsprüfungsgesellschaft um die 20 Prozent, während Sozialversicherung und Produktionsuntemehmen zum Teil deutlich mehr als 30 Prozent aufweisen.

In Kapitel 7 dieser Untersuchung ist der Dauer der Beschäftigung auf der Basis der quantitativen Analyse ein ausführlicher Teil der Untersuchung gewidmet.

6.5.4 Untemehmensaustritte nach Gründen 1984, 1986, 1988 für alle Organisationen

Die folgende Tabelle inventarisiert die Gründe der Unternehmensaustritte in den Jahren 1984, 1986 und 1988.

Tab. 9: Organisationsaustritt nach Gründen in den Jahren 84, 86, 88 in Prozenten

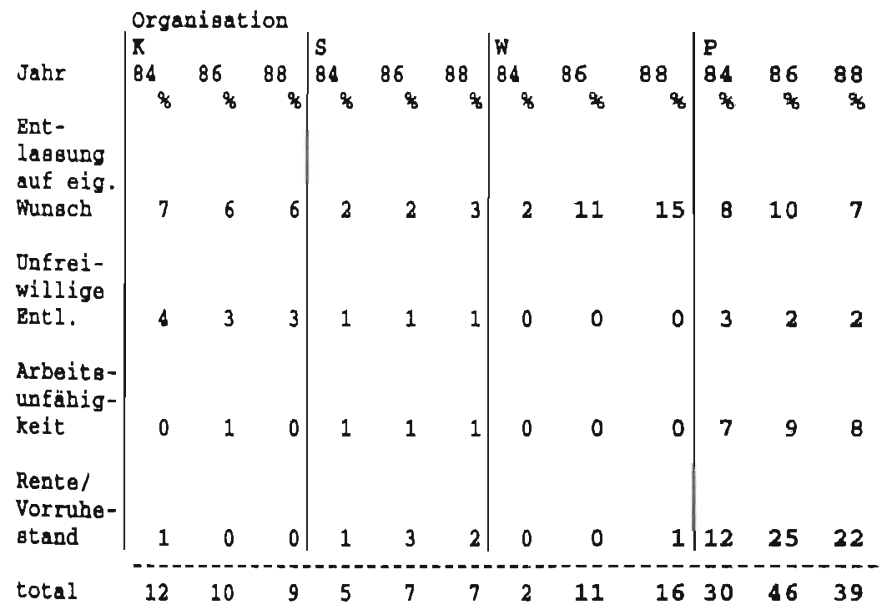

Zeichenerklärung: $\mathrm{K}=\mathrm{Krankenhaus;} \mathrm{S}=$ Sozialversicherung; $\mathrm{W}=\mathrm{Winsch}$ aftsprüfungsuntemehmen; $\mathrm{P}=$ Produktionsuntemehmen

Die in obiger Tabelle aufgezeigten Organisationsaustritte entsprechen weitgehend den Erwartungen. Entlassungen auf eigenen Wunsch fallen für die Wirtschaftsprüfungsgesellschaft für die Jahre 1986 und 1988 etwas hoch aus. Dies entspricht dem Mobilitätsmuster, das bei professionellen Bürokratien zu erwarten ist. Ein geringer Teil der Mobilität dürfte der Fusionsproblematik zuzuschreiben sein. Es besteht aus mehr interorganisationaler statt 
intraorganisationaler Mobilität. Organisationsaustritt aus Gründen der Arbeitsunfähigkeit ist beim Produktionsunternehmen erwartungsgemäB höher als bei Organisationen, deren Aktivität dienstleistungsorientient ist. Auffallend ist der hohe Anteil von Personen, die von der Vorruhestandsregelung Gebrauch machen. Dies erklärt sich aus Tatsache, daß bewußt dieses Personalführungsinstrument in Anspruch genommen wird mit dem Ziel, den Personalbestand zu reduzieren.

\subsubsection{Kernaktivitäten im Personalbereich}

In diesem Abschnitt geht es um die Kemaktivitäten der Personalabteilungen in den untersuchten Organisationen. Gefragt wurde nach dem Zeitanteil, der auf die Kernaktivitäten der Personalabteilung entfällt. Die Existenz einer Personalabteilung wird als Indikator gewertet für die Entwicklung eines internen Arbeitsmarktes (Pfeffer/Cohen, 1984) und die Institutionalisierung von Beschäftigungsmustem. Die Angaben basieren auf Einschätzung der Gesprächspartner.

Stellenwerbung und Selektion von Personal wird in den großen Organisationen (Krankenhaus und Produktionsuntemehmen) mit einem Prozentanteil von 20 wahrgenommen, während er in den kleineren Organisationen (Wirtschaftsprüfungsgesellschaft und Sozialversicherung) bei 15 bzw. 10 Prozent liegt. Hier zeigt sich, daß von der Tendenz größere Unternehmen mehr Wert auf Stellenwerbung und Selektion von Mitarbeiter legen können.

Die Aktivität "Stellenzuweisung bzw. -veränderung" beläuft sich auf 20 Prozent für drei Organisationen. Die Sozialversicherung weicht mit 60 Ptozent hier deutlich von den übrigen Organisationen ab. Dies ist zu erklären mit den Automatisierungs- und Reorganisationsbemühungen, die die Organisation gegenwärtig beherrschen.

Die Personalplanung als Kemaktivität macht bei allen Organisationen 10 Prozent aus mit Ausnahme der Wirtschaftsprüfungsgesellschaft, bei der der Anteil bei 20 Prozent liegt.

Bei der Einschätzung der Kernaktivität "Personalbeurteilung/-begleitung" entfält ein deutlich höherer Zeitanteil auf die Personalbegleitung. Hier besteht bei allen Organisationen eine stärkere Priorität als bei der Personalbeurteilung, die noch relativ unterentwickelt ist, d. h. sie ist wenig institutionalisiert bzw. steht am Beginn der Formalisierung. Zum Teil wird die Personalbeurteilung in den verschiedenen Organisationen allein für Mitarbeiter durchgefuhrt, die noch nicht lange in der Organisation anwesend sind. Mit 10 Prozent ist Personalbeurteilung und -begleitung im Vergleich zu den anderen Organisationen relativ unterentwickelt.

Eine Bewertung von 20 bzw. 25 Prozent trifft für die Kemaktivität Arbeitsbewertung/ Gehaltsfragen zu. Eine Ausnahme im Vergleich dieser Kemaktivität zwischen den untersuchten Organisationen stellt die Sozialversicherung dar mit 10 Prozent. Bei der Sozialversicherung ist das Zahlenmaterial vorsichtig zu interpretieren, da die Kernaktivitätenverteilung stärker unter dem Eindruck gegenwärtiger Prioritätensetzung steht. 
Tab. 10: Kernaktivitäten im Personalbereich in Prozenten

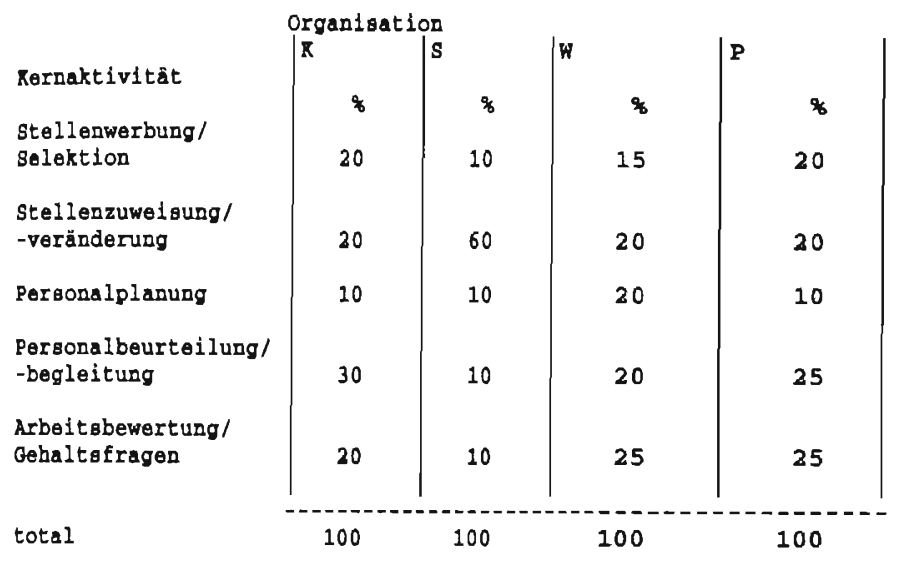

Zeichenerklärung: $\mathrm{K}=\mathrm{Krankenhaus;} \mathrm{S}=\mathrm{Sozialversicherung;}$

$\mathrm{W}=$ Wirtschaftsprüfungsunternehmen; $\mathrm{P}=$ Produktionsunternehmen

\subsection{Organisationskennzeichen zur Bestimmung von Personalpolitik im Rahmen der sozialen Unternehmensführung}

Hier soll der Frage nachgegangen werden, inwieweit die in diesem Kapitel aufgezeigten personalwirtschaftlichen Organisationsmerkmale typisch sind für die soziale Untemehmensfïhrung im weiten und mit der jeweiligen Organisationskonfiguration im engeren Sinne.

Soziale Untemehmensführung läßt sich nach Dunlop (1958) definieren als die Gesamtheit von Regeln, die aus der Arbeitnehmer-Arbeitgeberbeziehung hervorgehen. Diese Definition ist breiter angelegt als die im Rabmen der Definition interner Arbeitsmärkte als Regeln institutionalisierten Funktionierens interner Arbeitsmärkte. Ein Teil dessen, was den Inhalt sozialer Untemehmensführung ausmachen kann, kam in diesem Kapitel zum Ausdruck. In einer empirischen Untersuchung (Paauwe, 1989), die die soziale Unternehmensführung im Kontext der Organisationskonfigurationen von Mintzberg zum Gegenstand hat, zeigt sich eine Bestätigung dafür, daß die soziale Untemehmensführung typisch ist für die jeweilige Organisationskonfiguration. Dies gilt sowohl für die Maschinenbürokratie als auch die professionelle Bürokratie.

Mit Bezug auf die hier untersuchten Maschinenbürokratien kommt die soziale Unternehmensführung auf planmäBige und formale Weise zustande, wobei die Personalabteilung einen entscheidenden Anteil hat. Dominant ist bei dieser Organisationskonfiguration die Initiative von der 'Organisationsspitze'. Im Stadium der Beratung ist vielfach ein großer 'Kreis' von Betroffenen einbezogen. Bei der professionellen Bürokratie ist nach Paauwe 
die Entstehung der sozialen Untemehmensführung abhängig von der Größe des Unternehmens und dem Umfang des Regelungsbedarfs. Nach der hier vorliegenden Untersuchung wird dieser Aspekt bestätigt. Darin liegt auch der Schlüssel für eine direktere Beeinflussung von Seiten der Betroffenen in professionellen Bürokratien. Änderungen der sozialen Unternehmensführung sind vielfach mehr unternehmensextern als -intern hervorgerufen.

\subsection{Zusammenfassende Bewertung}

In Kapitel 3 wurden allgemeine und beschäftigungsorientierte Kennzeichen der Organisationskonfigurationen vorgestellt. Sie dienten als theoretische Basis für die Überprüfung der empirischen Befunde, die in diesem Kapitel behandelt wurden.

Im Rahmen einer zusammenfassenden Bewertung ergeben sich Anhaltspunkte für eine weitgehende Übereinstimmung der Kennzeichen bei den in der Realität angetroffenen Organisationen. Damit wird an Hand von Kennzeichen aufgezeigt, was zunächst als Annahme in die Untersuchung eingegangen ist. Ebenfalls lassen sich Hinweise für die Übereinstimmung ableiten in Bezug auf die Beschäftigungsdauer, die Länge der Jobleitem, die Orientierung (Kosten versus Wissen), die organisationsbezogene Mobilität, die Art der hauptsächlichen Rekrutierung, die Qualifikationsinhalte, die Kontrollform, die Thematik Zentralisierung/Dezentralisicrung und die Einstellung der Arbeitnehmer zur Arbeit.

Keine direkten empirischen Anhaltspunkte ließen sich ableiten bezüglich der Frage der Automation, der Strategie, des Führungsstils.

Die folgenden Resultate der quantitativen empirischen Analyse ergänzen die in diesem Kapitel aufgezeigten Eigenschaften der Organisationen und die Resultate der qualitativen Analyse. 


\section{Empirische Befunde der Infrastrukturanalyse interner Arbeitsmärkte}

Im vorangegangenen Kapitel wurden empirische Befunde der qualitativen Erhebung in Bezug auf die untersuchten Organisationen beschrieben. Gegenstand dieses und des folgenden Kapitels sind die empirischen Befunde der quantitativen Untersuchung. Im folgenden werden die Hypothesen explizit verbunden mit den Analysen. Neben den Hauptwegen der Untersuchung werden Seitenwege eingeschlagen, soweit dies vom Untersuchungsmaterial her zu rechtfertigen ist. Die Interpretation der Ergebnisse bezieht sich darum auch auf andere zu erklärende Gegebenheiten. Diese gehen damit über die in den Hypothesen zum Ausdruck kommende Sachverhalte hinaus.

Im Vordergrund dieses Kapitels steht die Infrastrukturanalyse interner Arbeitsmärkte in vier Organisationen, die in unterschiedlicher Weise Merkmale interner Arbeitsmärkte verkörpern. Ein Aspekt dabei betrifft die Mobilität innerhalb von Organisationen des internen Arbeitsmarktes. Diese wird gekennzeichnet durch die Dynamik der internen Organisation. Mobilität ist Schlüsselkategorie (Kalleberg/Berg, 1987) für die Beurteilung des Zugangs, des intemen 'Umsatzes' und des Abgangs von Personal (Buttler u. a., 1978). Mobilität kann verschiedene Formen annehmen, die hier kurz erläutert werden sollen. Im Rahmen dieser Analyse findet eine Beschränkung auf Formen intraorganisationaler Mobilität staat. Als Ergänzung zur Mobilitätsanalyse umfaBt dieses Kapitel außerdem eine Analyse der Arbeitnehmerbindung sowie der Beschäftigungsdauer von Arbeitnehmem in den jeweiligen Organisationen. Damit folgen wir einer Forderung von Althauser/Kalleberg (1990) und Rosenbaum (1990). Diese Autoren kritisieren, daß die Laufbahnprofile bislang ohne Zeitdimension betrachtet wurden. Die Einbeziehung der Verbleibzeit von Arbeitnehmem auf Funktionen ist eine Forderung, abgeleitet aus der dynamischen Mobilitäts- bzw. Karriereforschung (Schein, 1986).

1 Mobilität ist aufzufassen als freiwillige, d. h. mit dem Willen des betroffenen Arbeitnehmers zustande gekommene Versetzung, soweit sie den Übergang zu einer anderen Funktion in der Organisation beinhaltet. Mobilität wird aufgefaßt als 'Beförderung'. Sie kann in diesen Fällen sowohl in horizontaler als auch in vertikaler Richtung erfolgen (Hypothese 1).

2 Eine andere Analyse konzentrien sich auf die Gehaltszuwachsrate als Indikator fur Arbeitnehmerbindung. Danach ist in Maschinenbürokratien eine höhere Gehaltszuwachsrate zu erwarten als in professionellen Bürokratien. Die Arbeitsplatzstruktur bzw. die Gehaltsstruktur ist auf intemen Arbeitsmärkten so angelegt, daB die Arbeitnehmerbindung in Form höherer Gehaltszuwachsraten bei Maschinenbürokratien und geringerer Giehaltszuwachsraten bei professionellen Bürokratien zum Ausdruck kommt. Die hier durch- 
geführte Analyse basiert auf Veränderungen der Gehaltszuwachsraten im Jahresvergleich. Hier liegt die Vorstellung zugrunde, daß in Maschinenbürokratien ein höherer Gehaltszuwachs als vergleichbar bei professionellen Bürokratien $\mathrm{zu}$ konstatieren ist, aufgrund einer in Maschinenbiurokratien deutlicheren Tendenz der Arbeitnehmerbindung (Hypothese $3)$.

3 Schließlich wird in einem dritten Teil die Beschäftigungsdauer von Arbeitnehmem innerhalb der Organisationen analysiert. Dem liegt ein Konzept des Funktionswechsels innerhalb der Organisation zugrunde. Über die Funktionswechsel wird die Verbleibzeit des Arbeitnehmers auf bestimmten Funktionen erschlossen. Daran ist ein längerer Beobachtungszeitraum gekoppelt, der über den in der Stichprobe erfaßten Vierjahreszeitraum hinaus geht. Ziel dieser Analyse ist die breitere Erfassung von Funktionsänderungen (Hypothesen 4 und 5).

Dieses Kapitel gliedert sich wie folgt: zunächst wird eine Übersicht des Datenmaterials gegeben, verbunden mit einer Erläuterung der in den verschiedenen Untersuchungskontexten eingegangenen Variablen. Danach schließen sich die Regressionsanalysen an, deren Ergebnisse im Anschluß kurz interpretiert werden. Den einzelnen Schritten liegen unterschiedliche statistische Verfahren zugrunde, die im jeweiligen Kontext erläutert werden.

\subsection{Beschreibung der Variablen der Infrastrukturanalyse}

Ziel dieses Abschnitts ist es, die Variablen der Mobilitätsanalysen kurz einzuführen, zu erläutem und auf die jeweilige Analyseform zu beziehen. Unterschieden wird dabei zwischen abhängigen und unabhängigen Variablen. Zuiächst gilt die Aufmerksamkeit den abhängigen Variablen.

\subsubsection{Die abhängigen Variablen der Infrastrukturanalyse}

Die Infrastrukturanalyse besteht - wie angedeutet - aus mehreren Teilen. Damit wird den unterschiedlichen Erscheinungsformen der Mobilităt mit dem darauf ausgelegten Datenmaterial Rechnung getragen.

Der Mobilitätsform (Beförderung) entspricht die abhängige erste Variable. Sie gehı als Variable für intraorganisatorische Mobilität in die Analyse ein. 'MOB' steht für 'Beförderung'. Die Variable steht in enger Verbindung mit Hypothese 1. Die Variable wurde erzeugt durch die Erfassung sämtlicher freiwilliger intemer Mobilitätsvorgănge, d. h. Befördenungen von Arbeitnehmern im Zeitraum von fünf Jahren im Rahmen der Stichprobe. Beförderungen unterscheiden sich von Funktionswechseln, die Gegenstand der Beschäftigungsdaueranalyse sind, dadurch, daß bei Beförderungen ein 'Aufstieg' verbunden ist in Form eines höheren Gehaltes, einer aussagekräftigeren Funktionsbezeichnung bzw. bei Personalverantwortung eine größere Kontrollspanne. Beim Wechsel der Funktion wird jede Funktionsänderung im Rahmen der Untersuchung erfaßt, ohne dabei auf die Art der Arbeitsplatzänderung zu schauen. Die folgende Tabelle gibt eine Übersicht über die Zahl und Verteilung der Beförderungen. 
Tab. 11: Verteilung der Zahl der Befördenungen auf die untersuchten Organisationen (MOB) zum Erhebungszeitpunkt 1989

\begin{tabular}{|c|c|c|c|c|c|c|}
\hline \multirow[t]{3}{*}{ Organisation } & \multicolumn{6}{|c|}{ Anzahl der BefBrderungen } \\
\hline & 0 & \% & $\geq 1$ & $1 \%$ & tota. & $1 \%$ \\
\hline & 69 & (92) & 6 & (8) & 75 & $(100)$ \\
\hline $\begin{array}{l}\text { Wirtschafte- } \\
\text { prüfungeges. }\end{array}$ & 75 & (97) & 2 & (3) & 77 & $(100)$ \\
\hline $\begin{array}{l}\text { 8ozialver- } \\
\text { eicherung }\end{array}$ & 49 & (68) & 27 & $(36)$ & 76 & $(100)$ \\
\hline $\begin{array}{l}\text { Produktions- } \\
\text { betrieb }\end{array}$ & 53 & $(68)$ & 25 & (32) & 78 & $(100)$ \\
\hline Total & 246 & $(80)$ & 60 & $(20)$ & 306 & $(100)$ \\
\hline
\end{tabular}

Aus der Tabelle läßt sich ein Verhältmis von Nichtmobilität zu Mobilität von 4:1 ableiten. Mobilitätsvorgänge sind auf zwanzig Prozent der erfaßten Fälle im Rahmen der Stichprobe konzentriert. Betrachtet man die Mobilitätsvorgänge nach der Verteilung auf die Organisationstypen der 'professionellen Bürokratie' (Krankenhaus und Wirtschaftsprïfungsgesellschaft) beziehungsweise der 'Maschinenbürokratie' (Sozialversicherung und Produktionsbetrieb), so fällt auf, daß bei letztgenannten deutlich mehr Beförderungen zu verzeichnen sind. Daraus läßt sich vorläufig die SchluBfolgerung ableiten, daß in der Maschinenbürokratie im Vergleich zur 'professionellen Bürokratie' mehr intraorganisationale Mobilität vorhanden ist.

Ausdruck der Arbeitnehmerbindung auf internen Arbeitsmärkten ist die Koppelung von Funktion und Gehalt (Dunlop, 1957; Piore, 1972 u. 1978; Schein, 1986). Bei Maschinenbürokratien isı zu erwarten, daß im Laufe der Beschäftigungszeit die durchschnittlichen Gehaltszuwächse deutlicher zunehmen als bei professionellen Bürokratien. Die zum Zwecke dieser Analyse geschaffene Variable (SALONTW) geht als weitere abhängige Variable in die Berechnungen ein.

Gehaltszuwachsentwicklung (SALONTW) basiert auf den durchschnittlichen Zuwachs des Bruttomonatsgehaltes von Jahr zu Jahr im Vierjahreszeitraum. Diese Variable steht im Zusammenhang mit der Prüfung von Hypothese 3. In den Fällen, in denen Arbeitnehmer im Laufe des Untersuchungszeitraums rekrutiert wurden, d. h. in dem der Zeitraum der Beobachtung kürzer (kürzer als 4 Jahre) war, wurden die Durchschnittswerte des entsprechenden kürzeren Zeitraums berücksichtigt.

Soweit die Rekrutienung im Jahre 1988 erfolgte, wurden auf Grund der fehlenden jahrbezogenen Vergleichsmöglichkeiten organisationsspezifische Durchschnittswerte allein für diese Fälle berücksichtigt. Bei Personen, die über 10000 Gulden brutto pro Monat verdienen und deren Gehalt konkret nicht zu erfahren war (vielfach sind Gehaltsinformationen in oberen Bereichen der Hierarchie ein Tabu), wurde ein Gehaltszuwachs von durchschnittlich 500 Gulden per Jahr als Zuwachs berücksichtigt. 
Die Maßeinheit der Variable (SALONTW) bezieht sich auf Gulden. Das entsprechende Zahlenmaterial ist nicht inflationsbereinigt, da die Inflationsrate im Laufe des Untersuchungszeitraums für alle Organisationen dieselbe war. Die Berechnung der Durchschnittswerte ergibt sich aus der Differenz des Bruttomonatsgehaltes von 1988 und 1984 . geteilt durch die Zahl der erfaßten Jahre, in diesem Fall: vier. Analog wurden die Berechnungen für die Jahre, bei denen ein kürzerer Zeitraum zugrunde liegt, durchgeführt. Für das letzte Jahr (1988) wurden die zuvor berechneten Durchschnittswerte per Organisation auf der Grundlage von 278 Fällen berücksichtigt.

Per Organisation ergeben sich folgende Durchschnittswerte der Gehaltszuwachsentwicklung (in Gulden) auf der Basis von 278 Fällen:

Tab. 12: Durchschnittliche Gehaltszuwachsentwicklung nach Organisation

$\begin{array}{ll}\text { Krankenhaus: } & 100 \\ \text { Wirtachaftaprüfungsgesell schaft } & 179 \\ \text { Sozlalver icherung } & 229 \\ \text { Produktiongunternehmen } & 220 .\end{array}$

Diese Werte fanden Berücksichtigung bei neuen Rekrutierungen im Jahre 1988. Die Mittelwerte auf Basis von 306 Fällen finden sich in Tabelle 17/18. Sie weichen nicht von den genannten Werten ab.

\subsubsection{Die unabhängigen Variablen der Infrastrukturanalysen}

In diesem Abschnitt gilt es. die unabhängigen Variablen kurz vorzustellen. Bei dieser Gelegenheit sollen die Variablen soweit erforderlich an Hand empirischen Materials erläutert und interpretiert werden. Die Auswahl der Variablen basiert primär auf Litcratur sowie auf neuen theoretischen Überlegungen, z. B. die Berücksichtigung der Zeitkomponente bei Lautbahnprofilen. Die folgenden Variablen waren Gegenstand statistischer Analysen. Die Textnummerierung der zu erläuternden unabhängigen Variablen erfolgt in Anlehnung an Tab. 17/18.

3 Funktion (FKT) charakterisiert grob das Niveau der Tätigkeit, die Arbeitnehmer ausführen. Sämtliche Funktionen wurden im Rahmen der Stichprobe erfaßt und nach einem Hierarchiestufenmodell des Niederländischen Lohntechnischen Dienstes (1987) durch Bewertung einer Skala von acht Hierarchiestufen zugeordnet. In diesem Zusammenhang sollen kurz Anfangs- und Endwert des Hierarchiespektrums erläutert werden. Niveau 1 umschreibt das unterste Hierarchieniveau. Gemeint sind sehr einfache Tätigkeiten, die sich stïndig wiederholen, keine Ausbildung und keine Erfahrung erfordern, z. B. die Funktion der Küchenhilfe.

Mit Niveau 8 wird das hüchste Hierachieniveau beschrieben. Auf dieser Stufe sind Personen anzutreffen. die große Untemehmungen oder gleichwertige Einrichtungen leiten, beispielsweise der Direktor einer großen Untemehmung oder eines Krankenhauses. 
Tab. 13: Verteilung der Funktionen zum Erhebungszeitpunkt (1989) über Organisationen und Funktionsniveaus im Rahmen der Stichprobe

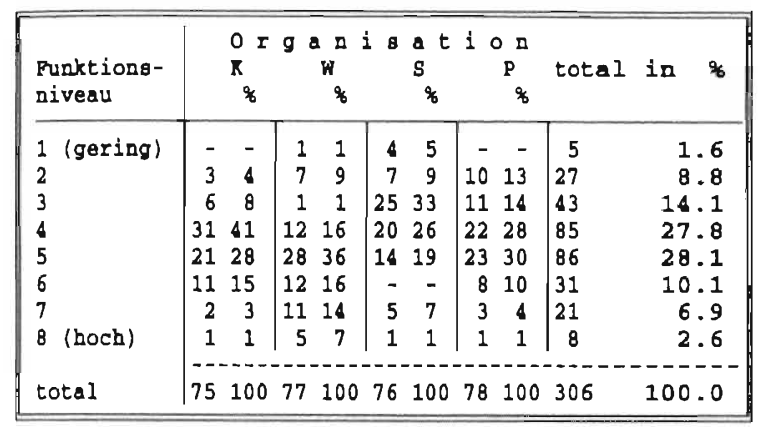

Erläuterung: K=Krankenhaus; $S=$ Sozialversicherung; $W=W$ irtschaftsprïfungsuntemehmen; $\mathrm{P}=$ Produktionsuntemehmen

Betrachtet man die Funktionsverteilung dieser Tabelle als Resultat einer geschichteten Stichprobe mit Bezug auf die verschiedenen Organisationsformen, so lassen sich unterschiedliche Tendenzen feststellen. Zu berücksichtigen bei der Interpretation ist die Komprimierung der Funktionen auf acht Funktionsniveaus. Gegenúber der Realität kann dies zu Verzermungen in der Verteilung führen. Die Funktionsniveauverteilung im Krankenhaus entspricht weitgehend der Nomalverteilung. Funktionskonzentrationen betreffen Niveau 4 mit 31 Besetzungen und Niveau 5 mit 2l Besetzungen. Bei Funktionen auf diesem Niveau handelt es sich vorwiegend um Pflegepersonal. Die Konzentration auf diesem Niveau und die Besetzung von Niveau 6 unterstreichen qua Tendenz den Charakter als professionelle Bürokratie, in der eine relativ hohe Anzahl Personen auf einem hohen Funktionsniveau anzutreffen ist. Die Wirtschaftsprüfungsgesellschaft wird den Kriterien der professionellen Bürokratie in dieser Hinsicht noch mehr gerecht. Hier zeichnet sich eine Polarität ab, einerseits mit einer Konzentration auf Funktionsniveau 2 bis 5 , sowie andererseits auf Funktionsniveau 6 bis 8.

Die Sozialversicherung wird in der Funktionsverteilung dem Image der Maschinenbürokratie gerecht. Hier sind die Funktionen deutlich auf Funktionsniveau 3 und 4 konzentriert. Diese Organisation zeigt in der Funktionsverteilung eine Baumstruktur, die nach oben durch einen spitzen Zuschnitt charakterisiert wird. Die Funktionsverteilung im Produktionsuntemehmen ist auf die mittleren Funktionsniveaus konzentriert wie dies bei den professionellen Bürokratien der Fall ist. Die Verteilung der Funktionsniveaus beim Produktionsunternehmen ist deutlich gekennzeichnet durch die demographische Entwicklung der Organisation. Die hohen Alterskategorien sind deutlich stärker ausgeprägt.

Mintzberg unterscheidet Tendenzen der Entwicklung (Zugkraft) von Organisationen. Einzelne Merkmalsausprägungen bringen dies zum Ausdruck. Hier stellt sich die Frage, inwieweit andere 'governance structures' anwesend sind. Wir basieren diese Tendenz auf die Verteilung der Funktionen innerhalb der Organisation. Beim Krankenhaus als professioneller Bürokratie besteht eine leichte Tendenz (Zugkraft) zur Maschinenbürokratic 
(Stevens, 1987). Beim Produktionsunternehmen geht die Tendenz (Zugkraft) in Richtung professioneller Bürokratie. Unterstützt wird dieser Trend durch die technologische Entwicklung (Kern/Schumann, 1984). Dies wird deutlich an der relativ hohen Besetzungszahl von Funktionsniveau 4 und 5. Die übrigen Organisationen korrespondieren nahezu mit den idealtypischen theoretischen Organisationsformen. So entspricht die Wirtschaftsprifungsgesellschaft den Kriterien der professionellen Bürokratie mit einer deutlichen Konzentration der Funktionen auf den hohen Funktionsniveaus, während die Sozialversicherung dem Wesen der Maschinenbürokratie am besten nahe kommt.

4 Fluktuation (VERL) umschreibt die durchschnittliche Fluktuation per Organisation. Diese wurde berechnet nach der Formel von Bergeijk/de Grip (1986). Die Formel lautet:

$$
M=K(Q / E+I / B) \times 100 \%
$$

wobei $M$ den Veränderungsprozentsatz, $Q$ den Personalabgang, I die Zahl der Neueintritte und $\mathrm{E}$ den durchschnittlichen Personalbestand repräsentiert. Kleine Werte konstituieren eine geringe Fluktuation, hohe Werte ein höheres $\mathrm{MaB}$ an Fluktuation.

5 Bei der Variable 'Geschlecht' (GESL) steht Wert 1 für "männlich", Wert 2 steht für "weiblich".

6 Die Variable 'Weiterbildung' (OP) gibt Auskunft über die betrieblichen Weiterbildungsaktivitäten, deren Resultate im Betrieb direkt oder indirekt Anwendung finden sollen. Erfaßt werden im Rahmen der Stichprobe verschiedene Formen der Weiterbildung im Fünfjahreszeitraum. Differenziert wird zwischen folgende Kriterien: keine Weiterbildung, exteme Weiterbildung und betriebsspezifische Weiterbildung. Der geringste Wert entspricht dem Kriterium "keine Weiterbildung". Die Skalierung erfolgt von 1 bis 3. Welche Verteilung sich im einzelnen ergibt, ist der nachfolgenden Tabelle zu entnehmen.

Tab. 14: Verteilung der Formen der Weiterbildung

\begin{tabular}{|c|c|c|c|c|c|c|c|}
\hline \multirow[t]{3}{*}{ Organisation } & \multicolumn{7}{|c|}{ Formen der Weiterbildung } \\
\hline & \multicolumn{2}{|c|}{$\begin{array}{l}\text { obne Wei- } \\
\text { terbildung }\end{array}$} & \multicolumn{2}{|c|}{$\begin{array}{l}\text { mit exter. } \\
\text { w.-bildung }\end{array}$} & \multicolumn{2}{|c|}{$\begin{array}{l}\text { mit betriebs- } \\
\text { opezifischer } W .\end{array}$} & \multirow[t]{2}{*}{ Total } \\
\hline & & $\%$ & & $\%$ & & $\$$ & \\
\hline Krankenhaus & 67 & $(25)$ & 7 & $(32)$ & 1 & $(6)$ & 75 \\
\hline $\begin{array}{l}\text { Mirtochafte- } \\
\text { prúfungoges. }\end{array}$ & 67 & $(25)$ & 9 & (41) & 1 & (6) & 77 \\
\hline $\begin{array}{l}\text { Sozialver- } \\
\text { sicherung }\end{array}$ & 63 & (24) & - & - & 13 & (82) & 76 \\
\hline $\begin{array}{l}\text { Produktiona- } \\
\text { betrieb }\end{array}$ & 71 & $(26)$ & 6 & $(27)$ & & $(6)$ & 78 \\
\hline Total & 268 & $(100)$ & 22 & $(100)$ & 16 & $(100)$ & 306 \\
\hline
\end{tabular}

Erläuterung: Die Prozentwerte befinden sich in Klammern. 
Die Tabelle zeigt bei Sozialversicherung (Maschinenbürokratie) einen großen Umfang ( 82 Prozent) an betriebsspezifischen Weiterbildungsmaßnahmen. Bei den professionellen Bürokratien genießt die exteme Weiterbildung gegenüber der betriebsspezilischen deutliche Priorität. Dies hängt zusammen mit der Aus- und Weiterbildung, die auf ein transferierbares Qualifikationniveau hinzielt. Im Krankenhaus sind das Pflegepersonal und technisches Personal, im Wirtschaftsprüfungsuntemehmen sind das zukünftige Wirtschaftsprüfer. Auch im Produktionsunternehmen dominiert im Rahmen der Stichprobe die externe vor der internen Weiterbildung. Diese 'Verzemung' hängt von der Tatsache $a b$, daß Lemaktivitäten 'on the job' nicht explizit erfaßt werden im Gegensatz zu Formen extemer Weiterbildung. Aufgrund der qualitativen Informationen der Untersuchung ergeben sich Hinweise bei dieser Organisation dafür, daß die Lernaktivitäten 'on the job' in Wirklichkeit das Ausmaß extemer Weiterbildung weit überschreiten.

7 Variable 'Dienstjahre' (JID) umschreibt die Beschäftigungsdauer von Arbeitnehmern in der jeweiligen Organisation.

8 Das 'Schulausbildungsniveau' (OPLNIVO) (schulischer bzw. beruflicher Ausbildungsabschluß) bei Eintritt in die Organisation wird in Form einer fünfstufigen Skala dokumentiert. Der Wert I charakterisiert das niedrigste Niveau in Form eines einfachen Schulabschlußes, der Wert 5 das höchste in Form eines Universitätsabschlusses.

Tab. 15: Schulausbildungsniveau der im Rahmen der Stichprobe erfaßten Arbeitnehmer

\begin{tabular}{|c|c|c|c|c|c|c|c|c|c|c|}
\hline \multirow{2}{*}{$\begin{array}{l}\text { Schulaus- } \\
\text { b1ldungs- } \\
\text { niveau } \\
1+2\end{array}$} & \multicolumn{9}{|c|}{ 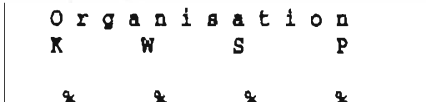 } & \multirow{2}{*}{$\begin{array}{l}\text { total } \\
\% \\
18.9\end{array}$} \\
\hline & 8 & 10 & 4 & 5 & 19 & 24 & 27 & 35 & 58 & \\
\hline 3 & 50 & 67 & 23 & 30 & 37 & 49 & 24 & 31 & 134 & 43.8 \\
\hline 5 & $\begin{array}{r}14 \\
3\end{array}$ & $\begin{array}{r}19 \\
4\end{array}$ & $\begin{array}{l}36 \\
14\end{array}$ & $\begin{array}{l}47 \\
18\end{array}$ & $\begin{array}{r}13 \\
7\end{array}$ & $\begin{array}{r}17 \\
9\end{array}$ & $\begin{array}{r}19 \\
8\end{array}$ & $\begin{array}{l}21 \\
10\end{array}$ & $\begin{array}{l}82 \\
32\end{array}$ & $\begin{array}{l}26.8 \\
10.5\end{array}$ \\
\hline total & 75 & 100 & 77 & 100 & 76 & 100 & 78 & 100 & 306 & 100.0 \\
\hline
\end{tabular}

Erläuterungen: l=einfache Schulausbildung; 2=einfache Schulausbildung; 3=mittlere Schul-/Berufsausbildung; 4=höhere Schul-/Berufsausbildung; 5=Universitätsausbildung $\mathrm{K}=$ Krankenhaus; $\mathrm{W}=$ Wirtschaftsprüfungsuntemehmen; $\mathrm{S}=$ Sozialversicherung; $\mathrm{P}=$ Produktionsunternehmen

Die Interpretation des tabellarischen Zahlenmaterials bestätigt Tendenzen, die im Kontext anderer Variablen schon angedeutet wurden. Auch an Hand dieser Variable läßt sich die jeweilige Organisationskonfiguration erkennen. Das mittlere Ausbildungsniveau ist beim Krankenhaus sehr stark ausgeprägt. Noch deutlicher wird der Charakter der professionellen Bürokratie bei der Wirtschaftsprïfungsgesellschaft. Die höchste Merkmalsausprägung bei der Wirtschaftsprüfungsgesellschaft ist auf Niveau 4 anzutreffen. Die Sozialversicherung zeigt zusammen mit dem Produktionsunternehmen eine Konzentration der Verteilung der 
Ausbildungsniveaus auf den unteren Niveaus. Dies ist als Indikator für die Maschinenbürokratien zu werten.

9 Die Variable 'Typ' (TYPE) differenzient zwischen professioneller Bürokratie und Maschinenbürokratie. Wert 1 steht für die 'professionelle Bürokratie', Wert 2 für die Maschinenbürokratie (Mintzberg, 1979).

10 Außerdem geht die Variable 'Lebensalter'(LEEF) in die Regressionsberechnung ein.

11 Als weitere unabhängige Variable wurde die Variable (PA) geschaffen. Sie steht für die aus Mintzbergs Organisationsaufteilung in 'Parts' abgeleitete Mobilität zwischen den 'Parts'. Jede der Linien in der folgenden Skizze repräsentiert potentielle Mobilität. Es geht also um 'Inter-Part-Mobilität' innerhalb der Periode von Eintritt in die Organisation bis 1988. Part-Mobilität ist unabhängig von der Mobilitätsform 'Beförderungen' zu sehen. Part-Mobilität kann nicht als eine Teilmenge der Beförderungen aufgefaßt werden. PartMobilität berücksichtigt die Erfassung bzw. Verschiebung der Arbeitnehmerzugehörigkeit von einem Part zum anderen. Der Vergleich der Partzugehörigkeit selzt einen längeren Beobachtungszeitraum voraus.

Abb. 25: Mobilität zwischen Parts

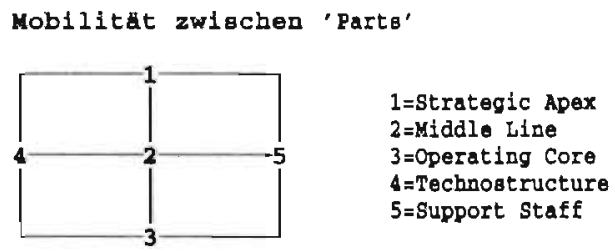

Brklarung der Part-Mobllitätgniveaus:

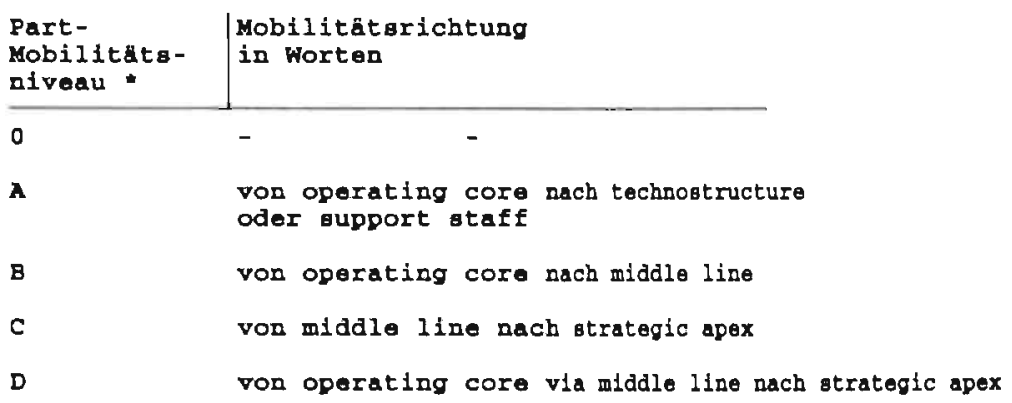

* Die Logik der 'part-mobility' schließt auch die umgekehrte Mobilitätsrichtung ein, die jedoch von der Häufigkeit des Auftretens äußerst begrenzt ist.

Ein hohes $\mathrm{Maß}$ an Mobilität wird durch den Aufstieg eines Arbeitnehmers vom 'operating core' zum 'strategic apex' repräsentiert. Ein geringes Maß an Mobilität wird durch den Wechsel vom 'operating core' zur 'technostucture' oder 'support staff' wiedergegeben. 
Es geht - wie gesagt - bei dieser Variable um die Erfassung von Mobilitätsvorgängen zwischen den Parts. Nicht erklärt werden können damit Mobilitätsvorgänge innerhalb der Parts, die aufgrund von Funktionsketten und den entsprechenden Funktionshierarchien zum Teil wesentlich komplexer sind.

Tab. 16: Verteilung der Fälle von Part-Mobilität (PA)

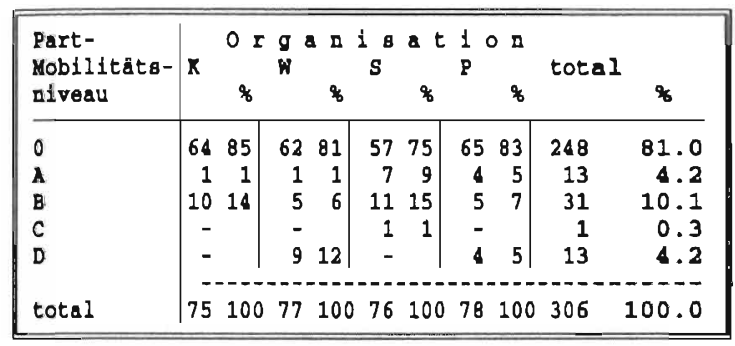

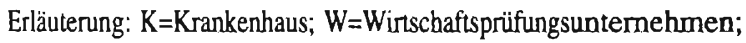
$S=$ Sozialversicherung; $P=$ Produktionsunternehmen

In obiger Tabelle kommen die Fälle von Part-Mobilität per Organisation und Mobilitätsniveau zum Ausdruck. Part-Mobilitätsniveau 0 dokumentiert die Fälle, in denen keine Part-Mobilität stattgefunden hat. Bei Mobilitätsniveau A handelt es sich um Mobilitätsschritte zwischen den Parts 3, 4 sowie 5 und umgekehrt. Die geringe Ausprägung dieses Mobilitätsniveaus läßt den Schluß zu, daß Arbeitnehmer Mobilität innerhalb der Parts entwickeln und meistens in den Parts verbleiben, in denen sie sich zu Beginn ihrer Organisationszugehörigkeit befunden haben. Dies gilt besonders für Personal, das in den Parts 'technostructure' und 'support staff' beschäftigt ist. Relativ stark ausgeprägt mit 7 ist die Zahl der 'Partwechsler' bei der Sozialversicherung. Diese Erscheinung ist zu erklären durch den recht flexiblen Personaleinsatz auf unteren Hierarchieniveaus. So läßt sich bei dieser Organisation nachweisen, daß beispielsweise Schreibkräfte einen PartWechsel vollziehen. Sie wechseln von einer 'support staff'-Position zu einer Anlemfunktion innerhalb des 'operating core'.

Part-Mobilitätsniveau B zeigt für alle Organisationen eine relativ starke Besetzung. Dieses Niveau kennzeichnet den Übergang vom 'operating core' zur 'middle line'. Das Ergebnis der deutlichen Ausprägung entspricht den Erwartungen. Diese Form des 'Partwechsels' korrespondiert mit dem 'klassischen Mobilitätsfeld', in dem die meisten Fälle von PartMobilität vorkommen.

Die Wahrscheinlichkeit, bei Part-Mobilitätsniveau C, das ist der Übergang von 'middle line' zum 'strategic apex', stark ausgeprägte Häufigkeiten anzutreffen, ist im Rahmen einer Stichprobe und in Bezug auf den Erhebungszeitraum von fünf Jahren gering. Darüberhinaus muß man sich vor Augen führen, daß die pyramidale Form der Organisationen in diesem Bereich spitzer zuläuft, d. h. die Besetzung mit Personal hierarchieaufwärts tendenziell abnimmt. 
Ähnliches läßt sich auch für Part-Mobilitätsniveau D sagen. Damit ist die höchste Form der Part-Mobilität angesprochen. Es geht dabei um den Übergang von 'operating core' über 'middle line' zum 'stategic apex'. Mit neun Fällen bei der Wirtschaftsprüfungsgesellschaft ist diese Mobilitätsform recht deutlich entwickelt. Dahinter steht das Phänomen, daß Wirtschaftsprüfer nach Jahren erfolgreicher Tätigkeit ihren Arbeitnehmerstatus gegen den des Untemehmers eintauschen. Sie werden 'Partner' (Mituntemehmer) innerhalb ihres Unternehmens. Die relativ hohe Zahl der Fälle von Part-Mobilität hängt zum einen mit der Expansion des Unternehmens zusammen, insbesondere die Errichtung von Außenstellen. Zum anderen ist die Chance, alle Parts zu durchlaufen relativ groß, da der hierarchische Funktionsabstand von Part zu Part für Wirtschaftsprüfer gering ist.

Die Mobilität zwischen Parts stellt eine grobe Analyse der intraorganisationalen Mobilität dar, da ein großer Teil der Mobilitätsbewegungen sich innerhalb der Parts auf mehr oder weniger langen Funktionsketten vollzieht. Die Mobilitätsanalyse zwischen Parts erscheint vor allem deshalb sinnvoll, da mit dieser Mobilitätsform der Zugang zu einer differenzierteren segmentationsanalytischen Betrachtung (Sengenberger, 1987) ermöglicht wird (Schwan/Soeters, 1991c). Bislang wird die arbeitsmarkttheoretische Literatur beherrscht von zwei Segmenten bzw. drei (Doeringer/Piore, 1971; Lutz/ Sengenberger, 1978). Die Part-Mobilität berücksichtigt fünf Segmente bzw. Organisationsteile. Wir beschränken uns hier allein auf die Einführung der Variable (PA) als unabhängige Variable, ohne weiter auf die Analyse der Part-Mobilität einzugehen, die in Schwan/Soeters (1991c) ausgeführt wurde.

Auf Grund einer hohen Multikollinearität (Tabachnick und Fidell, 1989) wurden folgende Variablen, die ursprünglich in den Regressionsberechnungen einbezogen waren, nicht berücksichtigt. Dabei handelt es sich einerseits um die Variable 'Wachstum', eine Variable, die die durchschnittliche Personalentwicklung im Fünfjahreszeitraum (Erhebungszeitpunkt 1989) für jede Organisation angibt:

- Krankenhaus: Negativwachstum mit 2 Prozent

- Wirtschaftsprüfungsgesellschaft: Wachstum mit 34 Prozent

- Sozialversicherung: Wachstum mit 13 Prozent

- Produktionsunternehmen: Negativwachstum mit 5 Prozent.

Andererseits geht es um die Variable 'Demographie', die die demographische Struktur des Personalaufbaus per Organisation wiedergibt sowie die Variable 'Sektor', die eine Differenzierung zwischen öffentlichen und privaten Organisationen bewirken sollte. Die Multikollinearität stellt sich unter diesen Variablen in Bezug auf die Variable 'Funktion' ein.

\subsection{Werteübersicht der Variablen der Mobilitätsanalyse}

In diesem Abschnitt werden die statistischen Werte der Variablen, die in die Mobilitätsanalyse eingehen, kurz vorgestellt. Dies geschieht durch drei Formen beschreibender statistischer Analyse. Dabei handelt es sich um das Resultat der (Con-) Descriptivanalyse, der Varianzanalyse sowie der Pearsonkorrelation. 


\subsubsection{Werteübersicht der Variablen nach der (Con-)Descriptivanalyse}

Im folgenden schließt sich eine Werteübersicht der hier vorgestellten Variablen an, aus der Mittelwerte, Standardabweichung, Minimum und Maximum sowie die Schiefheit der Verteilung (Skewness) zu entnehmen sind. Diese ist über die SPSS-Software Standards definiert. Die Anzahl der Fälle beläuft sich auf insgesamt 306.

Tab. 17: Variablenbeschreibung: Infrastrukturanalyse

\begin{tabular}{|c|c|c|c|c|c|}
\hline $\begin{array}{l}\text { Variablen } \\
\text { (niedrlg=) }\end{array}$ & Mittelwert & $\mid \begin{array}{l}\text { Standard } \\
\text { abwelchung }\end{array}$ & Minimum | & Maximum & Skewn. \\
\hline $\begin{array}{l}1 \mathrm{MOB} \\
\text { (wenig Beförder.) }\end{array}$ & 0.1 & 0.4 & 0 & 2 & 1.67 \\
\hline $\begin{array}{l}2 \text { SALONTW } \\
\text { (geringe Gehalts- } \\
\text { differenz }\end{array}$ & 181.6 & 193.4 & 0 & 1648 & 3.5 \\
\hline $\begin{array}{l}3 \text { PKT } \\
\text { (niedrige Funkt.) }\end{array}$ & 4.4 & 1.5 & 1 & 8 & 0.14 \\
\hline $\begin{array}{l}\text { VERL } \\
\text { (niedrige Flukt.) }\end{array}$ & 5.4 & 1.1 & 3.6 & 6.5 & 0.79 \\
\hline $\begin{array}{l}5 \text { GESL } \\
\text { (männl1ch) }\end{array}$ & 1.2 & 0.4 & 1 & 2 & 0.89 \\
\hline $\begin{array}{l}6 \text { OP } \\
\text { (ohne Weiterbdg.) }\end{array}$ & 0.2 & 0.5 & 0 & 2 & 2.83 \\
\hline $\begin{array}{l}7 \text { JID } \\
\text { (geringe Begchäf- } \\
\text { tigungadauer) }\end{array}$ & 11.8 & 9.5 & 0 & 38 & 0.56 \\
\hline $\begin{array}{l}8 \text { OPLNIVO (gering } \\
\text {-es schulausbil- } \\
\text { dungeniveau) }\end{array}$ & 3.2 & 1.0 & 1 & 5 & 0.21 \\
\hline $\begin{array}{l}9 \text { TYPE } \\
\text { (prof. Bürokr.) }\end{array}$ & 1.5 & 0.5 & 1 & 2 & 0.00 \\
\hline $\begin{array}{l}10 \text { LEBP } \\
\text { (niedriges } \\
\text { Lebensalter) }\end{array}$ & 37.8 & 10.3 & 19 & 61 & 0.20 \\
\hline $\begin{array}{l}11 \text { PA (geringe } \\
\text { partbezogene Mo- } \\
\text { bilitat) }\end{array}$ & 0.4 & 1.0 & 0 & 4 & 2.42 \\
\hline
\end{tabular}

$\mathrm{N}=306$

Erläuterung: ${ }^{*}=$ in Gulden; Verhältnis Gulden:DM = 1:0.89; Skewn. $=$ Skewness 


\subsubsection{Varianzanalyse Infrastrukturanalyse}

Die folgende Tabelle zeigt die Resultate der Varianzanalyse mit Differenzierung nach Organisationen.

Tab. 18: Ergebnisse der Varianzanalyse Infrastruktur

\begin{tabular}{|c|c|c|c|c|c|c|}
\hline \multirow[t]{2}{*}{$\begin{array}{l}\text { Varlablen } \\
\text { (niedrlg=) }\end{array}$} & \multicolumn{6}{|c|}{ 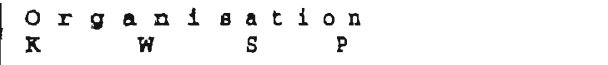 } \\
\hline & $M i t$ & $t \cdot 1$ & wert & $\theta$ & $P$ & Signif. \\
\hline $\begin{array}{l}1 \text { MOB } \\
\text { (wenig Mobilitat) }\end{array}$ & 0.1 & 0.0 & 0.4 & 0.3 & 15.1 & $\pitchfork *$ \\
\hline $\begin{array}{l}2 \text { SALONTW } \\
\text { (geringe Gehalta- } \\
\text { differenz) }\end{array}$ & 100.6 & 179.1 & 229.3 & 220.2 & 7.4 & t** \\
\hline $\begin{array}{l}3 \text { PRT } \\
\text { (niedrige } \\
\text { Punktion) }\end{array}$ & 4.5 & 5.1 & 3.7 & 4.2 & 12.7 & $\neq \#$ \\
\hline $\begin{array}{l}4 \text { VERL } \\
\text { (niedrlge Flukt.) }\end{array}$ & 6.5 & 5.5 & 6.0 & 3.6 & - & - \\
\hline $\begin{array}{l}5 \text { GBSL } \\
\text { (mannlich) }\end{array}$ & 1.5 & 1.3 & 1.4 & 1.0 & 17.8 & $\pitchfork \bullet$ \\
\hline $\begin{array}{l}6 \text { OP (ohne } \\
\text { Weiterbildung) }\end{array}$ & 0.1 & 0.1 & 0.3 & 0.1 & 4.0 & 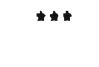 \\
\hline $\begin{array}{l}7 \text { JID } \\
\text { (geringe Beschaf- } \\
\text { tigungsdauer) }\end{array}$ & 10.8 & 8.1 & 9.7 & 18.7 & 23.9 & $\pitchfork \bullet$ \\
\hline $\begin{array}{l}8 \text { oPLwIVo (gering } \\
\text {-es Schulausbil- } \\
\text { dungsniveau) }\end{array}$ & 3.1 & 3.8 & 2.9 & 3.0 & 11.3 & 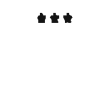 \\
\hline $\begin{array}{l}9 \text { TYPE } \\
\text { (prof. Bürokr.) }\end{array}$ & 1.0 & 1.0 & 2.0 & 2.0 & - & - \\
\hline $\begin{array}{l}10 \text { LERF } \\
\text { (nledrige日 } \\
\text { Lebenoalter) }\end{array}$ & 36.3 & 35.8 & 35.2 & 44.0 & 14.1 & $\pitchfork *$ \\
\hline $\begin{array}{l}11 \text { PA (geringe } \\
\text { partbezogene Mo- } \\
\text { bilität }\end{array}$ & 0.3 & 0.6 & 0.4 & 0.4 & 1.4 & n. \\
\hline
\end{tabular}

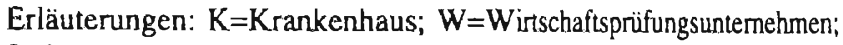
$\mathrm{S}=$ Sozialversicherung; $\mathrm{P}=$ Produktionsuntemehmen; $\mathrm{F}=\mathrm{F}$-Statistik; -=organisationsspe zifische Ausprägung; ${ }^{* *} p<0.001 ;{ }^{*}=$ in Gulden; n. $s .=$ nicht signifikant

Bei den Variablen 'Fluktuation' und 'Type' wurde keine F-Statistik berechnet, weil es sich bei diesen Variablen um kollektive Organisationsscores handelt, die zu einem späteren Zeitpunkt in die Analyse eingefügt wurden. 
Die Zahlen geben über die Mittelwerte einen ersten Eindruck der quantitativen Besetzung der Variablen. Iher bieten sich erste Ansätze zu einem organisationsspezifischen Vergleich an. Das Zahlenmaterial bestätigt auf der Basis von Mittelwerten die Erwartung einzelner Variablen. Im Rahmen der ausführlichen Definition der Variablen und der Übersicht der Subausprägungen erfährt diese Tabelle eine ausführliche Besprechung. Wir beschranken uns an dieser Stelle auf diesen Hinweis, ohne weiter im Detail auf die Mittelwerte einzugehen.

\subsubsection{Signifikante Pearsonkorrelationen der Mobilitătsanalyse}

Der folgende Schrit der Datenanalyse konzentriert sich auf die Darstellung der Pearsonkorrelationen. In der Anlage (Tab. 19 bis 21) befinden sich die Pearsonkorrelationen zu den hier benutzten Variablen.

Die Pearsonkorrelationen geben allein einfache Zusammenhänge wieder. Thr Wert besteht darin, einen ersten Eindruck zu bekommen und eine Voraussetzung zu schaffen für eine qualitativ höherwertige Analyse, die in den folgenden Abschnitten ausgefuihrt werden soll. Die Pearsonkorrelationen werden neben den Regressionsanalysen weitere Verwendung finden. Auf eine Verbalisienung der signifikanten Korrelationen soll deshalb hier verzichtet werden. Bei den Pearsonkorrelationen wurde der Datensatz differenziert in Gesamtdatensatz und Teildatensatz. Letzterer bezieht sich auf die professionellen Bürokratien bzw. auf Maschinenbürokratien.

\subsection{Erweiterte statistische Analysen}

Die folgenden Analyseschritte bestehen aus der Einbeziehung der signifikanten Pearsonkorrelationen (die jeweiligen unabhängigen Variablen korrelieren mit der "MOB"-Variable und der "SALONTW"-Variable als abbängige Variablen), aus Regressionsanalysen mit den (in der Einleitung erläuterten) abhängigen Variablen unter Berücksichtigung der unabhängigen Variablen. Zugrunde liegen hier Betawerte. Darüberhinaus gilt es, kurz auf die Bedeutung der Aussagen der Regressionsanalyse anhand der Variablen einzugehen. Bei allen Regressionsanalysen wurden die Werte der Konstanten berechnet. Aus Gründen der Übersichtlichkeit wird jedoch darauf verzichtet, diese in die Ergebnisdarstellung aufzunehmen. Es geht allein um Zusammenhänge mit absoluten Werten.

Bei den folgenden statistischen Analysen wird der Datensatz differenziert. Einerseits wird die Totalerhebung ( $\mathrm{N}=306)$ berïcksichtigt andererseits die spezifizierte Aufteilung nach 'Organisationstyp' der professionellen Bürokratien und Maschinenbürokratien.

\subsubsection{Intraorganisationale Mobilität in Form der Beförderungsanalyse}

Die 'Beförderungsanalyse' entspricbt der Mobilitätsform, bei der Beförderungen von Arbeitnehmem auf freiwilliger Basis im Vordergrund stehen.

Kern dieser Analyse bildet die Regressionsberechnung. Durch die Einwirkung einer Vielzahl von Variablen auf die abhängige Variable entstehen signifikante Zusammenhänge auf qualitativ höherem Niveau durch Anwendung einer anspruchsvolleren statistischen 
Methode unter Beriicksichtigung von Interaktionseffekten. Die Pearsonkorrelation drückt einfache Zusammenhänge aus. Berücksichtigt wurden allein signifikante Pearsonkorrelationen. Die Resultate werden zeigen, inwieweit die festgestellten einfachen Pearsonkorrelationen auf dem Niveau der Regressionsanalyse vereinbar sind. In den Fällen, in denen aufgrund der Variablenstruktur dies möglich ist, wird ergänzend versucht, die Ergebnisse bei dualen Variablenwerten durch eine Diskriminantenanalyse zu bestätigen. Die methodische Verantwortung des Einsatzes der Diskriminantenanalyse erfolgte in Abschnit 5.3.

Die Beförderungsanalyse ermöglicht Einsichten in die intraorganisationale Mobilität der Arbeitnehmer in den vier untersuchten Organisationen. Mit dieser Analyse wird Hypothese I überprüft.

Tab. 22: Beförderungsanalyse: alle Organisationen; Pearsonkorrelation, Regressionsanalyse (MOB als abhängige Variable)

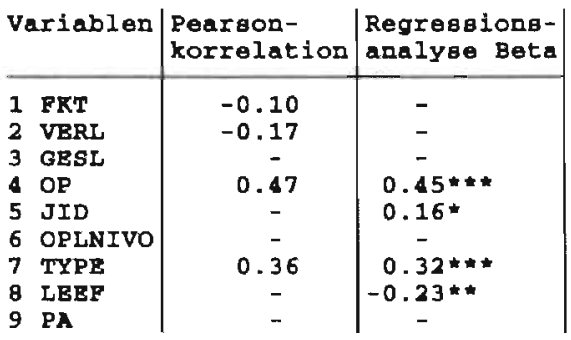

$r^{2}=0.34 ;$ adj. $r^{2}=0.32 ; N=306$

Signifikanz: ${ }^{*} p<0.05 ;{ }^{* *} p<0.01 ; * * * p<0.001$

In der Reihenfolge der signifikanten unabhängigen Variablen der Regressionsanalyse ergeben sich folgende Zusammenhänge:

1 Betriebsspezifische Weiterbildung erhöhı gegenüber fehlender bzw. extemer Weiterbildung die Chance zu Beförderungen.

Für beide Organisationskonfigurationen trifft dieses Ergebnis zu. Betriebliche Weiterbildung, ob betriebsintern oder -extern durchgeführt, ermöglicht die Chance auf Beförderung. Arbeitnehmer haben die Möglichkeit, eine höhere und damit besser bezahlte Funktion einzunehmen. Damit wird die Arbeitsmotivation erhöht und ein Anreiz zum Verbleib in der Organisation geboten (Rosenbaum, 1984; Bills, 1987; DiPrete, 1987; Wolfs, 1992). Betriebsinterne Weiterbildung erhöht die Chance auf Beförderung tendenziell noch mehr als die Formen externer Weiterbildung. Damit wird der organisationsspezifische Charakter der Weiterbildung angesprochen, der typisch für inteme Arbeitsmärkte ist.

2 In den Maschinenbirrokratien kommen durchschnittlich mehr Beförderungen vor als in den professionellen Bürokratien.

Dieses Ergebnis (Hypothese 1) zeigt einen Unterschied in der Art der Beschäftigungsverhältnisse auf. Aufgrund der differenzierteren Funktionsstruktur in Maschinenbürokratien 
und einer höheren intemen Mobilitätsdynamik und der Lage der 'ports of entry' lassen sich in Bezug auf Maschinenbürokratien mehr Beförderungen erfassen als vergleichsweise bei professionellen Bürokratien. Mit dem oben ausgedrückten Ergebnis kann Hypothese I bestätigt werden.

3 Mit steigendem Lebensalter von Arbeitnehmem nimmt die Chance auf Beförderungen ab.

Mit diesem signifikanten Zusammenhang wird ein Resultat aus Rosenbaums (1984) empirischer Untersuchung bestätigt. Man kann davon ausgehen, daß die organisatorischen Lemprozesse an ein bestimmtes Lebensalter gekoppelt sind. Bei hohem Lebensalter nimmt die Bereitschaft, Humankapitalinvestitionen vorzunehmen, ab. Umgekehrt wird deutlich, daß in 'jungen Jahren' mehr Chancen auf Beförderung bestehen (Frühstarterthese) (Rosenbaum, 1984; Brüderl, 1991).

4 Mit zunehmender Dienstzeit von Arbeimehmem innerhalb der Organisation steigt auch die Zahl der Beförderungen.

Die Chance auf Befördenung basiert auf der Jobleiterstruktur. Die Dauer des Beschäftigungsverhältnisses ermöglicht es, die ganze Jobleiter von unterschiedlicher Länge zu durchlaufen (Brüderl/Diekmann/Preisendörfer, 1989). Von der Tendenz gibt die Länge der Dienstzeit die Gelegenheit, mehrfach befördert zu werden. Umgekehrt setzt eine Beförderung eine längere Dienstzeit voraus.

Zwischen den Variablen 'Dienstzeit' und 'Lebensalter' besteht ein Interaktionseffekt. Dahinter stehen zwei für interne Arbeitsmärkte zutreffende Erscheinungen. Zum einen eine abnehmende Chance zur Befördenung bei fortgeschrittenem Lebensalter, zum anderen die Zunahme der Beförderungen mit zunehmender Dienstzeit.

Die zur Ergänzung durchgeführte Partialkomelation bestätigt unter Kontrolle der übrigen Variablen die Signifikanz des Ergebnisses. Die Partialkorrelation ist eine besondere Analyse, die nicht mit der Pearsonkormelation verwechselt werden darf. So ergibt sich ein Korrelationswert von 0.12 für MOB/JD unter Berücksichtigung der Kontrollvariablen LEEF, OP und TYPE bei einem Signifikanzniveau von 0.02. Für die Korrelation MOB/ LEEF wurde ein Wert von -0.13 bei einem Signifikanzniveau von 0.01 berechnet für die Kontrollvariablen OP, JID und TYPE.

Keine signifikante Beziehung besteht zwischen den Variablen Funktion, Fluktuation, Geschlecht, Schulniveau, Part-Mobilität und der abhängigen Variable.

Um weiteren differenzierteren Aufschluß über das Ergebnis der Regressionsanalyse zu erhalten, wurde auf der Grundlage des bei der Regessionsanalyse benutzten Datensatzes eine Diskriminantenanalyse (siehe Anlage, Tab. 23) durchgeführt. Die Diskriminantenanalyse bestätigt analysenspezifisch exakt die Ergebnisse der Regressionsanalyse. Inwieweit diese Ergebnisse bei der Betrachtung der Differenzierung in Organisationstypen bestätigt werden können, werden die folgenden Analysen zeigen. 
Wir kehren nun zur regessionsanalytischen Berechnungen der Mobilitätsanalyse zurück Bei den professionellen Bürokratien wird sowohl auf die Regressions- als auch Diskriminantenanalyse verzichtet, da bei dieser Organisationskonfiguration insgesamt nur acht Beförderungen zu verzeichnen sind. Aufgrund der geringen Anzahl können die statistischen Ergebnisse als nicht zuverlässig betrachtet werden. Für die Organisationsformen der Maschinenbürokratie ergeben sich die folgenden Ergebnisse:

Tab. 24: Beförderungsanalyse: Maschinenbürokratie Pearsonkorrelation, Regressionsanalyse (MOB als abhängige Variable)

\begin{tabular}{ll|c|c|} 
Variablen & $\begin{array}{l}\text { Pearson- } \\
\text { korrelation }\end{array}$ & $\begin{array}{l}\text { Regressions- } \\
\text { analyse Beta }\end{array}$ \\
\hline 1 & FRT & - & - \\
2 VERL & - & - \\
3 GESL & - & - \\
4 OP & - & $0.55^{\star \star *}$ \\
5 & JID & - & $0.22^{\star}$ \\
6 OPLNIVO & - & - \\
7 LEEF & -0.19 & $-0.27 \star$ \\
8 PA & - & -
\end{tabular}

$\mathrm{r}^{2}=0.34 ;$ adj. $\mathrm{r}^{2}=0.32 ; \mathrm{N}=153$

Signifikanz: * $p<0.05 ;{ }^{* *} p<0.01 ; * * * p<0.001$

In der Reihenfolge der signifikanten Zusammenhänge läßt sich die Bedeutung des Zahlenmaterials in Worte fassen:

1 In den Maschinenbürokratien hängen betriebsspezifische Weiterbildung und Beförderungen eng zusammen.

Die Maschinenbürokratien gehören zu den Organisationskonfigurationen, bei denen betriebsspezifische Aus- und Weiterbildung dominiert. Interne Lemprozesse sind gekoppelt an Beförderungen bei aufeinander abgestimmten Funktionen (Spilerman, 1977; Agyris/ Schön, 1978).

2 Arbeitnehmer mit hohem Lebensalter in Maschinenbürokratien haben wenig Beförderungschancen.

Rosenbaum (1990) spricht vom 'age timetable', d. h. einer Koppelung von Funktion und Alter. Mit vierzig Jahren ist nach seinen Erkenntnissen der kritische Zeitpunkt erreicht, bei dem die Beförderungschancen deutlich abnehmen.

3 Mit der Länge der Dienstzeit innerhalb der Maschinenbürokratien nimmt die Zahl der Beförderungen zu.

Beförderangen sind bei Maschinenbürokratien als Belohnung für die Bemühung zu sehen, daß Arbeitnehmer sich dem organisatorischen Lemprozeß stellen. Die Art der Funktionsabfolge, die Arbeitnehmer durchlaufen, ist an eine bestimmte Betriebszugehörigkeit 
gebunden. Auf diese Weise wird von Seiten der Arbeitgeber eine Art 'Betriebsbindung' erreicht (Bills, 1987).

Auch hier läßt sich ein Interaktionseffekt ableiten. Die Analyse der Partialkorrelationen ergeben für die Maschinenbürokratien die folgenden Ergebnisse. MOB/JID korrelieren mit einem Wert von 0.16 bei einem Signifikanzniveau von 0.03 unter Berücksichtigung der Kontrollvariablen VERL, FKT, OP, LEEF, OPLNIVO. Für die Variablen MOB/L.EFF ergibt sich ein Korrelationswert von -0.19 bei einer Signifikanz von 0.01 . Als Kontrollvariablen wurden die Variablen FKT, VERL, JID, OPLNIVO und OP einbezogen.

Kein signifikanter Verband läßt sich feststellen bei den Variablen: Funktion, Fluktuation, Geschlecht, Schulausbildungsniveau und Part-Mobilität.

Bei der Diskriminantenanalyse (Anlage, Tabelle 25) mit Bezug auf den obigen Datensatz werden ebenfalls die Ergebnisse der Regressionsanalyse analysenspezifisch bestätigt.

\subsubsection{Analyse nach Gehaltszuwachsentwicklung}

Diese Analyse konzentrier sich auf die Gehaltszuwachsentwicklung als MaBstab für die Arbeimehmerbindung in den unterschiedlichen Organisationskonfigurationen (Hypothese 3). Bevor die Interpretation vorgenommen wird, gilt es, die quantitativen Ergebnisse zu betrachten. Bei dieser Analyse wird die Variable MOB als exogene Variable gebraucht. Das heiBt, daB es sich um eine eigenständige Analyse handelt mit SALONTW als abhängiger Variable. Da bei dieser das Verhältnis zur Variable MOB interessiert, wurde diese als unabhängige Variable in die Analyse einbezogen.

Tab. 26: Gehaltszuwachsanalyse: total; Pearsonkorrelation, Regressionsanalyse (SALONTW als abhängige Variable)

\begin{tabular}{l|c|c|} 
Variablen & $\begin{array}{l}\text { Pearson- } \\
\text { korrelation }\end{array}$ & $\begin{array}{l}\text { Regreselone- } \\
\text { analyse Beta }\end{array}$ \\
\hline 1 MOB & 0.34 & $0.22 * \star *$ \\
2 PKT & 0.31 & $0.37 \star *$ \\
3 VBRL & -0.15 & - \\
4 GESL & -0.26 & - \\
5 OP & 0.32 & $0.16 * *$ \\
6 JID & - & - \\
7 OPLNIVO & 0.21 & - \\
8 TYPB & 0.22 & $0.23 * \star *$ \\
9 LEEF & 0.12 & -
\end{tabular}

$r^{2}=0.30 ; \operatorname{adj} . r^{2}=0.28 ; N=306$

Signifikanz: ${ }^{*} p<0.05 ;{ }^{* *} p<0.01 ;{ }^{* * *} p<0.001$

Die Tabelle weist folgende signifikanten Werte der Regressionsanalyse aus:

1 Ein hohes Funktionsniveau entspricht einer hohen Gehaltszuwachsrate in den untersuchten Organisationen. 
Das Ergebnis zeigt ein Verhältnis von Funktion und Gehalt auf. Mit zunehmendem Funktionsniveau und einem höheren Grad an Verantwortlichkeit korrespondiert die Gehaltsentwicklung.

2 Bei Maschinenbürokratien sind die Gehaltszuwachsraten stärker entwickelt als bei professionellen Bürokratien.

Dieses Resultat kann zurückgeführt werden auf die durchschnittlich höhere Altersbesetzung in den Maschinenbürokratien. Bei einer relativ langen Beschäftigungsdauer und die damit verbundene demographische Stellenbesetzungstruktur ergibt sich ein anderes Bild als im Vergleich zu den professionellen Bürokratien. Ebenfalls spielt das Rekrutierungsverhalten eine Rolle. Da der Zustrom von jungen Menschen bei den professionellen Bürokratien relativ größer ist, die qua Gehaltsstruktur auf unteren Gehaltsstufen beginnen, kommt der Alterseffekt verbunden mit einer relativ höheren Einordnung zum Tragen. Die Zahl der Austritte aus den Organisationen ist auf gleichbleibendem Niveau, so daß das Ergebnis dadurch nicht beeinflußt wird. Mit dem obigen Ergebnis kann Hypothese 3 als bestätigt angesehen werden.

3 Bei Beförderungen von Arbeitnehmern ist ein Gehaltszuwachs zu enwarten.

Hier handelt es sich um eine für interne Arbeitsmärkte (und nicht nur für diese) typische Beziehung. Funktionen sind an Gehältem gekoppelt mit in der Regel aufsteigender Tendenz. Hierdurch soll ein Motivationsanreiz (Elbaum, 1983; DiTomaso, 1988) bei Arbeitnehmern geschaffen werden sowie eine Bindung des Arbeitnehmers an die Organisation (Piore, 1972).

4 Bei einem hohen $\mathrm{Maß}$ an betriebsspezifischer Weiterbildung läßt sich ein Gehaltszuwachs erwarten.

Damit wird indirekt ein Ergebnis bestätigt, das die Koppelung zwischen Weiterbildung bzw. Beförderung zum Ausdruck bringt. Keine Beziehung besteht zwischen den Variablen: Flukruation, Dienstzeit, Schulausbildungsniveau, Lebensalter, Geschlecht und der abhängigen Variable. Welche Unterschiede sich bei den professionellen Bürokratien zeigen, geht aus der folgenden Tabelle hervor. Bei den beiden folgenden Analysen wird ein Seitenweg beschritten (Ergänzung zu Hypothese 3). 
Tab. 27: Gehaltszuwachsanalyse: prof. Bürokratien Pearsonkorrelation, Regressionsanalyse (SALONTW als abhängige Variable)

\begin{tabular}{l|c|c|} 
Variablen & $\begin{array}{l}\text { Pearson- } \\
\text { korrelation }\end{array}$ & $\begin{array}{l}\text { Regreasions- } \\
\text { analyoe Beta }\end{array}$ \\
\hline 1 MOB & 0.26 & $0.24 * \star *$ \\
2 FRT & 0.59 & $0.59 * * *$ \\
3 VERL & -0.26 & $-0.24 * *$ \\
4 GESL & -0.38 & - \\
5 OP & - & - \\
6 JID & 0.16 & - \\
7 OPLNIVO & 0.17 & - \\
8 LEBF & 0.25 & -
\end{tabular}

$r^{2}=0.45 ;$ adj. $r^{2}=0.41 ; N=151 ;$

Signifikanz: ${ }^{*} p<0.05 ;{ }^{* *} p<0.01 ;{ }^{* * *} p<0.001$

Die Tabelle weist folgende signifikanten Zusammenhänge von oben nach unten auf:

1 Ein hohes Funktionsniveau in professionellen Bürokratien entspricht einer hohen Gehaltszuwachsrate.

Damit wird das Ergebnis der Totalanalyse für die professionellen Bürokratien bestätigt.

2 Bei Beförderungen von Arbeitnehmem in professionellen Bürokratien ist ein Gehaltszuwachs zu erwarten.

Mit diesem Ergebnis wird das Ergebnis der Totalanalyse für die professionellen Bürokratien bestätigt.

3 Bei professionellen Bürokratien ist eine hohe Gehaltszuwachsentwicklung in der Rege] verbunden mit einer niedrigen Fluktuation.

Das Resultat unterstreicht die Tendenz zu steigenden Gehaltszuwächsen bei einer bestimmten Dauer der Beschäftigung. Damit wird der Anreiz-Beitrags-Bezug als Motivationsgrundlage, aber auch die Arbeitnehmerbindung zum Ausdruck gebracht. Dabei unterscheiden sich professionelle Bürokratien nicht wesentlich von Maschinenbürokratien. Eine hohe Fluktuation kann dazu beitragen, daß die Gehaltszuwachsentwicklung nicht bzw. nur in kleinen Schritten stattindet.

Keine Beziehung zwischen der abhängigen und der unabhängigen Variable lassen sich für folgende Variablen feststellen: Geschlecht, Weiterbildung, Dienstjahre, Schulausbildungsniveau und Lebensalter. Die Maschinenbürokratien bieten folgendes Bild

(Ergänzung zu Hypothese 3): 
Tab. 28: Gehaltszuwachsanalyse: Maschinenbürokratien; Pearsonkorrelation, Regressionsanalyse (SALONTW als abhängige Variable)

\begin{tabular}{l|c|c|} 
Variablen & \begin{tabular}{|c|} 
Pearson- \\
korrelation
\end{tabular} & $\begin{array}{l}\text { Regres8 } \\
\text { analyse Beta }\end{array}$ \\
\hline I MOB & 0.30 & $0.17 *$ \\
2 FRT & 0.27 & - \\
3 VERL & - & - \\
4 GBSL & - & $-0.20 *$ \\
5 OP & - & $0.27 *$ \\
6 JID & - & - \\
7 ORLNIVO & 0.32 & - \\
8 LEEF & - & -
\end{tabular}

$r^{2}=0.27 ;$ adj. $r^{2}=0.22 ; N=153$

Signifikanz: ${ }^{*} p<0.05 ;{ }^{* *} p<0.01 ;{ }^{* * *} p<0.001$

Die Tabelle weist folgende signifikanten Zusammenhänge in der Reihenfolge der Signifikanz auf:

1 Mit betriebsspezifischer Weiterbildung in Maschinenbürokratien ist ein Gehaltszuwachs verbunden.

Hier kommt die Anreiz-Beitragssituation zum Ausdruck, die betriebsinterne Lemaktivitäten belohnt. Unterstrichen wird damit ein wesentliches Kennzeichen intemer Arbeitsmärkte.

2 Frauen partizipieren im Vergleich zu Männem in geringerem Maße von Gehaltszuwächsen in Maschinenbürokratien.

Die geschlechtsspezifischen Differenzen zwischen Frauen und Männern in der Zunahme des Gehaltes werden hier deutlich (Tijdens, 1989; van der Burg, 1992). Sie stehen im Einklang mit Erkenntnissen von Kanter (1977), DiPrete/Soule (1988), die allerdings nicht nur auf Gehaltszuwächse beschränkt sind (Gijsel, de/Muysken/Wolfs, 1991).

3 Bei Beförderungen von Arbeitnehmern in Maschinenbürokratien ist ein Gehaltszuwachs zu erwarten.

Hier wird das Ergebnis der Totalanalyse für die Maschinenbürokratien bestätigt. Keine Beziehung zwischen der abhängigen Variable und den unabhängigen Variablen lassen sich in Bezug auf folgende Variablen feststellen: Funktion Fluktuation, Schulausbildungsniveau, Dienstzeit und Lebensalter.

7.3.3 Zusammenfassung der Ergebnisse der Beförderungs- und Gehultszuwachsanalyse

Im Rahmen dieser explorativen Studie ergeben sich die folgenden Resultate. Gegenstand der Beförderungsanalyse war intraorganisationale Mobilität von Arbeitnehmern innerhalb der beiden Organisationskonfigurationen. Die Befördenungsanalyse hat gezeigt, das betriebsspezifische Weiterbildung die Chance auf Beförderungen erhöht. Die Mobilitäts- 
dynamik ist in den Maschinenbürokratien stärker ausgeprägt als in professionellen Bürokratien. Im Rahmen langfristiger Beschäftigung sind mehrere Beförderungen möglich. Als Ergebnis läßt sich festhalten, daß die Zahl der Beförderungen in professionellen Bürokratien kleiner ist als in Maschinenbürokratien. Das Signifikanzpositivzeichen der Variable "TYPE" in Tabelle 22 spricht für die Bestätigung von Hypothese 1.

Die Ergebnisse der Gehaltszuwachsanalyse deuten darauf hin, daß in Maschinenbürokratien die Gehaltszuwachsraten stärker entwickelt sind als bei professionellen Bürokratien. Bestätigt wird bei dieser Analyse die Arbeitnehmerbindung, die in einem steigenden Einkommensprofil sowie in Beförderungen zum Ausdruck kommt. Die aparte Analyse für die jeweiligen Organisationskonfigurationen zeigt für die Maschinenbürokratien, daß das Ergebnis beeinflußt wird durch Beförderungen und betriebliche Weiterbildung. Bei den professionellen Bürokratien wird die Arbeitnehmerbindung erreicht durch Beförderungen und der Koppelung von Funktion und Gehalt. Hypothese 3 bezieht sich auf den Vergleich von Maschinenbürokratien und professionellen Bürokratien. Angesichts der Tatsache, daB bei Maschinenbürokratien höhere Gehaltszuwachsraten gefunden wurden, gilt Hypothese 3 als bestätigt. Anhaltspunkt dafür ist das Signifikanzpositivzeichen der Variable "TYPE" in Tabelle 26. Bei der Gehaltszuwachsanalyse zeigen sich Charakteristiken des internen Arbeitsmarktes, die bei beiden Organisationskonfigurationen zutreffen.

Die Beförderungs- bzw. die Gehaltszuwachsanalysen sollen hier um eine weitere Analyse ergänzt werden, bei der die Beschäftigungszeiten in den verschiedenen Organisationen Berücksichtigung finden.

\subsection{Analyse der Beschäftigungsdauer von Arbeitnehmern in den verschiedenen Organisationen}

Die Theorie intemer Arbeitsmärkte nennt als Kennzeichen intemer Arbeitsmärkte eine lange Beschäftigungsdauer von Arbeimehmem. Zugrunde liegt dem die Strategie der Arbeitnehmerbindung. Die Arbeitnehmerbindung trägt zum Erhalt des know hows eines Betriebes bei und befördert die Organisationsentwicklung. Damit ist eine wichtige Voraussetzung für den Erhalt und das Fortbestehen der Unternehmensorganisation gegeben (Schwan/Soeters, 1992).

Die Arbeitnehmerbindung wird nicht allein durch die Perspektive von betrieblicher Beschäftigungssicherheit erreicht, sondem auch durch die Perspektive des Fortkommens bzw. der Beförderung (Cole, 1979; Creedy/Whitfield, 1988). Eine darauf bezogene Organisationsstruktur Jäßt sich ebenfalls zu den Kennzeichen interner Arbeitsmärkte zählen. Innerbetriebliche Funktionswechsel stellen eine Ausprägung dieses Kennzeichens dar.

Was begründet nun ein steigendes Forschungsinteresse an der Beschäftigungszeit von Arbeitnehmem? Dazu läßt sich die Diskussion über die Optimalisierung von Firmenkonzepten und Organisationskennzeichen anführen. Der Vergleich von amerikanischen und japanischen Firmen hat Ouchi/Jaeger (1978) zur Konzeption der "Theorie Z" veranlaßı, die darauf hinausläuft, daß amerikanische Untemehmen positive Beschäftigungsmerkmale 
japanischer Unternehmen übernehmen (um noch 'excellenter' zu funktionieren). Diese Merkmale sind Charakteristiken des intemen Arbeitsmarktes, wovon die lange Beschäftigungsdauer eines der markanten Kriterien ist. Die Diskussion wurde in jüngster Zeit fortgeführt durch Beiträge von Deutschmann (1987) und Lincoln/Kalleberg (1990).

Aus gesamtwirtschaftlicher Sicht besteht ein Interesse, möglichst in einem frühen Stadium Strukturveränderungen zu konstatieren. Untersuchungen dieser Art beziehen sich deshalb auf den Vergleich verschiedener Nationen oder allein auf nationalem Niveau (Schasse, 1990; Wolfs, 1990; Kerkhoffs/Wolfs, 1991). Für Arbeitnehmer steht die Beschäftigungsstabilität im Vordergrund, für Arbeitgeber die Kosten, die mit Fluktuation zusammenhängen.

Im Rahmen von Arbeitsmarktuntersuchungen richtet sich das Interesse auf die Beschäftigungszeit und die Einkommenshöhe von Arbeitsnehmern innerhalb von Organisationen. Bislang war es schwierig, über Datenmaterial zu verfügen, das Aufschluß bietet über furmeninteme Mobilitätsvorgänge und Verbleibzeiten. Selbst bei entsprechendem Datenmaterial lassen sich die methodischen Probleme (Preisendörfer/ Wallaschek, 1989; Kerkhoffs/Wolfs, 1991) nicht so ausräumen.

Das aggregierte Datenniveau läßt oft keine Schlußfolgerungen auf das Organisationsniveau zu. Auch die Organisationskonfiguration, die wiederum typisch für interne Arbeitsmärkte ist (Soeters/Schwan, 1990; Althauser/Kalleberg, 1990), wird vielfach auf eine Variable reduziert. Organisationsstrukturen bieten Karrierechancen. Bei intemen Arbeitsmärkten handelt es sich um Jobleitern als ein strukturkonstiruierendes Moment (Baron et al., 1986; Pfeffer/Cohen, 1984). Rosenbaum (1990) kritisiert das Jobleiterkonzept mit dem Hinweis auf die Vernachlässigung der Zeitabhängigkeit beim Verbleib von Arbeitnehmem auf bestimmten Funktionen innerhalb der Jobleiter. In diesem Teil der Arbeit wollen wir dem Rechnung tragen.

\subsubsection{Die ergänzenden Variablen der Beschäftigungsdaueranalyse}

Ein Kennzeichen interner Arbeitsmärkte ist ein langfristiges Beschäftigungsverhälnis (Hall, 1986). Zum Zwecke der Analyse von Beschäftigungszeiten wurde die Variable (TLJD=Beschäftigungsdauer) geschaffen. Der Wert der Variable (TIJD) ergibt sich aus dem Quotient der Variable (JID=Jahre in Dienst) und der Variable (LEEF=Lebensalter). Auf diese Weise erfolgte eine Standardisierung der Variable zur Ausschaltung demographischer Effekte. Die Variable 'TIJD' erfullt die Rolle als abhängige Variable. 
Tab. 29: Breakdown der Variablen: 'Jahre in Dienst' (JD) und 'Beschäftigungsdauer' (TJD) für die untersuchten Organisationen

\begin{tabular}{|l|rr|rr|r||}
\hline Organisation & \multicolumn{2}{|c|}{ Yittelwert } & \multicolumn{2}{|l|}{ Standardabweichung } & Palle \\
& JID & TIJD & JID & TIJD & \\
\hline Krankenhaus & 10.8 & 0.27 & 7.7 & 0.16 & 75 \\
Wirtschaftsprg. & 8.1 & 0.20 & 8.1 & 0.18 & 77 \\
Sozialversicher. & 9.7 & 0.25 & 8.1 & 0.18 & 76 \\
Produktionsuntn. & 18.7 & 0.40 & 9.7 & 0.17 & 78 \\
\hline Insgesamt & 11.9 & 0.28 & 9.4 & 0.19 & 306 \\
\hline
\end{tabular}

Die Tabelle zeigt die Durchschnittswerte der Dienstjahre der Beschäftigten der vier Organisationen. Professionelle Bürokratien lassen eine geringere Zahl der Dienstjahre erwarten gegenüber den Maschinenbürokratien. Die Realität in Bezug auf die professionellen Bürokratien zeigl, daB die Unterschiede weniger groß sind als erwartet. Für das Krankenhaus trifft eine Verbleibdauer von nahezu 11 Jahren zu. Im Rahmen der Erwartungen beläuft sich die Zahl der durchschnittlichen Dienstjahre für die Wirtschaftsprüfungsgesellschaft. Die Sozialversicherung liegt ebenfalls im Rahmen der Erwartungen. Deutlich abweichend ist der Durchschnittswert des Produktionsunternehmens. Dieser repräsentiert ein hohes Niveau mit nahezu 19 Dienstjahren. Die Werte der Variable (TIJD) lassen in dieser Version nur beschränkt konkrete Aussagen zu.

Zudem wurde eine neue unabhängige Variable eingeführt, die in besonderer Weise über die Erfassung von Funktionswechsel in der Vergangenheit der Dauer des Verbleibs auf bestimmten Funktionen Rechnung trägt. Die Variable (FUWI) basiert auf Funktionswechsel. Funktionswechsel können bestehen aus Beförderungen, gezwungener oder freiwilliger, horizontaler und/oder vertikaler Mobilität. Es wird hierbei nicht nach der Qualität der Funktionswechsel (im Gegensatz zur Beförderungsanalyse) gefragt. Erraßt wird jede Funktionsänderung, die sich innerhalb der Organisation im Zeitablauf ergibt. Diese Form der Analyse hat einen umfassenderen Zeitraum zum Gegenstand sowohl was den Beginn der Beschäftigungszeit betrifft als auch von der Annäherung an die Gegenwart (1989) (Blossfeld, 1985). Um der erweiterten zeitlichen Sichtweise methodisch Rechnung zu tragen, wurde Gebrauch gemacht von der Ereignisanalyse, die in Zeitintervallen den Zustandswechsel (hier: Funktionswechsel) erfaßt (Blossfeld/Hamerle/Mayer, 1986; Mayer/Carroll, 1987). Die Variable 'FUWI' wurde standardisiert mit Hilfe der Variable 'Jahre in Dienst'.

Die Struktur des Datensatzes besteht aus einem für jeden Arbeitnehmer variierenden Eintritt in die Organisation und einer Paneldatenerfassung für den Zeitraum 1984-1989. Dabei handelt es sich um rechtszensierte Daten. Gegenüber den in der Stichprobe berücksichtigten fünf Jahren umfaßt diese Variable die Spanne vom Organisationseintritt, die bei jedem Arbeimehmer individuell variiert, bis zum Jahr 1984. Von diesem Jahr an bezieht sich der Datensatz auf die Gegebenheiten zum Jahresende des jeweils folgenden Jahres bis zum Jahr 1988. Da die quantitative Erhebung in der Mitte des Jahres 1989 erfolgte, 
wurde, soweit keine Funktionsänderung in diesem Jahr eingetreten war, unterstellt, daß die jeweilige Funktion bis zum Jahresende andauerte. Damit sollte ein maximaler Beobachtungszeitraum für Funktionswechsel erfaßt werden. Bei Arbeitnehmern, die eine kürzere Dienstzeit als die im Schema erfaßte hatten, wurde die kürzere Zeit bei den Berechungen berücksichtigt.

Abb. 26: Struktur des Datensatzes der Variable (FUWI) im Rahmen der Ereignisanalyse

$$
\text { Funktions-Brfassungszeitpunkte/-râume (Bpisoden) }
$$

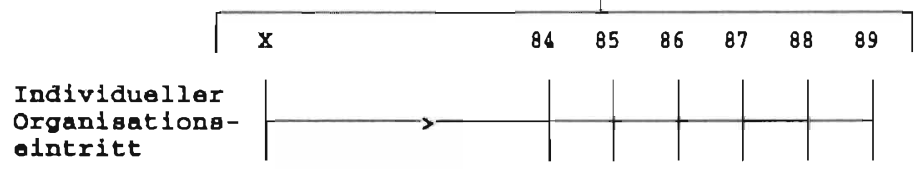

Nach dieser Strukturübersicht gilt es, die Verteilung der Funktionswechsel für die verschiedenen Organisationen zu betrachten. Die Tabelle basiert auf der Erfassung sämtlicher Funktionswechsel, soweit solche im definierten Zeitraum stattgefunden haben. Erfaßt wurden dabei alle möglichen Kombinationen. Die folgende Tabelle ermöglicht einen ersten quantitativen Eindruck. Sie gibt in kompakter Form die erfaßten Funktionswechsel wieder. Auf eine differenziertere Wiedergabe aller erfaßten Kombinationen wird hier aus Gründen der Übersichtlichkeit verzichtet.

Tab. 30: Funktionswechsel in der Langzeitbetrachung

\begin{tabular}{|c|c|c|c|c|c|c|c|c|c|c|}
\hline $\begin{array}{l}\text { Funktions- } \\
\text { wechsel }\end{array}$ & $\begin{array}{l}0 \\
K\end{array}$ & $\begin{array}{l}g \\
\%\end{array}$ & a $n$ & $\begin{array}{l}18 \\
*\end{array}$ & $\begin{array}{l}a t \\
s\end{array}$ & $\begin{array}{l}10 \\
\%\end{array}$ & $0 \mathrm{~g}$ & $\%$ & total & in \\
\hline P obne Fw. & 39 & 52 & 44 & 57 & 25 & 33 & 20 & 26 & 128 & 41.8 \\
\hline F mit 1 Fw. & 20 & 27 & 16 & 21 & 28 & 37 & 43 & 55 & 107 & 35.0 \\
\hline $\mathbf{F}$ mit 2 FW. & 16 & 21 & 17 & 22 & 18 & 24 & 13 & 17 & 64 & 20.9 \\
\hline \multirow{2}{*}{$\begin{array}{l}\text { F mit } 3 \mathrm{Fw} . \\
\text { total }\end{array}$} & - & - & - & & 5 & 6 & 2 & 2 & 7 & 2.3 \\
\hline & 75 & 100 & 77 & 100 & 76 & 100 & 78 & 100 & 306 & 100.0 \\
\hline
\end{tabular}

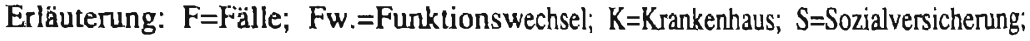
$\mathrm{W}=\mathrm{Wirtschaftsprüfungsunternehmen;} \mathrm{P}=$ Produktionsunternehmen

Die Tabelle zeigt die Funktionswechsel per Organisation. Unterschieden werden Kombinationen ohne Funktionswechsel, die rund 42 Prozent ausmachen gegenüber den Fällen von Funktionswechsel, die sich auf rund 58 Prozent der Fälle belaufen. Diese Fälle lassen sich weiter differenzieren in Kombinationen von 1 bis zu 3 Funktionswechseln. Diese Analyse entspricht besonderen methodischen Anforderungen. Von der Art der Anlage ist sie daher nicht vergleichbar mit der Beförderungsanalyse. Ergänzend zu den schon aus anderen Analysen bekannten Variablen sollen im folgenden die neuen Variablen kurz beschrieben werden. 
Tab. 31: Variablenbeschreibung: Beschäftigungsdaueranalyse

\begin{tabular}{l|c|c|c|c|c}
$\begin{array}{l}\text { Variablen } \\
\text { (niedrig=) }\end{array}$ & Mittelwert & $\begin{array}{l}\text { Standard- } \\
\text { abwelchung }\end{array}$ & Minimum & Maximum & Skewn. \\
\hline $\begin{array}{l}\text { 1 TIJD (kurze Be- } \\
\text { triebszugehörig.) }\end{array}$ & 0.3 & 0.2 & 0.0 & 0.7 & 0.11 \\
$\begin{array}{l}\text { 2 POWI (kein } \\
\text { Punktionswechsel) }\end{array}$ & 1.3 & 0.6 & 1.0 & 3.8 & 1.6
\end{tabular}

Skewn. $=$ Skewness

Ergänzend werden im folgenden die Variablen im Rahmen einer Varianzanalyse vorgestellt.

Tab. 32: Ergebnisse der Varianzanalyse der Betriebszugehörigkeit und Funktionswechsel

\begin{tabular}{|c|c|c|c|c|c|c|}
\hline \multirow[t]{2}{*}{$\begin{array}{l}\text { Variablen } \\
\text { (niedrlg=) }\end{array}$} & \multicolumn{6}{|c|}{$\begin{array}{l}0 r g a n i \\
R\end{array}$} \\
\hline & $M 1 t$ & e 1 . & $r t$ & & $\mathbf{P}$ & Signif. \\
\hline $\begin{array}{l}1 \text { TIJD (kurze Be- } \\
\text { triebszugehörigk.) }\end{array}$ & 0.3 & 0.2 & 0.3 & 0.4 & 20.0 & 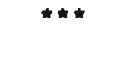 \\
\hline $\begin{array}{l}2 \text { POWI (kein } \\
\text { Funkt iongwechsel) }\end{array}$ & 1.3 & 1.3 & 1.5 & 1.4 & 1.9 & n. B. \\
\hline
\end{tabular}

Signifikanz: ${ }^{* * *} \mathrm{p}<0.001$; n.s. $=$ nicht signifikant

Die Variable (JD) 'Jahre in Dienst' liegt indirekt den Berechnungen zugrunde. Der 'breakdown' dieser Variable vermittelt einen ersten Eindruck über die durchschnittlichen 'Dienstjahre' per Organisation.

Aus dem deskriptiven Zahlenmaterial lassen sich partsbezogene durchschnittliche Beschäftigungszeiten bzw. -dauer ableiten (Anhang, Tab. 33). Diese Tabelle zeigt die durchschnittlichen Dienstjahre für die 'parts' auf. Daraus lassen sich folgende Trends ableiten. Für alle Organisationen mit Ausnahme der Wirtschaftsprüfungsgesellschaft läßt sich konstatieren, daß Teil 3 (operating core) im Gegensatz zu Teil 2 (middle line) kürzere Dienstzeiten aufweist. Dies spricht zum einen für den Eintrittsbereich zur Organisation, in dem relativ viel Mobilität stattfindet. Bei Teil 2 (middle line) liegt die Vermutung nahe, daß die Mobilität tendenziell abnimmt aufgrund der pyramidalen Form aber auch dem Ende von Jobleitem. Der 'part' 'middle line' enthält zahlreiche 'Funktionsparkplätze', es handelt sich dabei um Positionen, bei denen keine große Mobilitätserwartung besteht. Im Gegensatz zu 'technostructure' und 'support staff' liegen die Verbleibzeiten bei der 'middle line' relativ hoch. Der 'strategic apex-Part' läßt sich in etwa mit der Verbleibdauer im part 'technostructure' vergleichen.

\subsubsection{Die Resultate der Beschäftigungsdaueranalyse}

Im folgenden sollen die Ergebnisse der Regressionsberechnungen vorgestellt werden. Hierbei wird wieder die Unterscheidung gehandhabt: Totalanalyse, professionelle Bürokra- 
tien und Maschinenbürokratien. Nach jeder Analyseeinheit sollen die quantitativen Relationen kurz in Worte übersetzt werden. Im einzelnen lassen sich folgende Resultate verzeichnen:

Tab. 34: Beschäftigungsdaueranalyse: alle Organisationen Pearsonkorrelation (soweit signifikant), Regressionsanalyse (TLID) als abhängige Variable)

\begin{tabular}{l|c|c|} 
Variablen & $\begin{array}{c}\text { Pearson- } \\
\text { korrelation }\end{array}$ & $\begin{array}{c}\text { Regressions- } \\
\text { analyse Beta }\end{array}$ \\
\hline 1 FKT & - & $0.16 *$ \\
2 VERL & -0.32 & $-0.21 \star$ \\
3 GESL & -0.28 & - \\
4 OP & - & - \\
5 FUHI & -0.75 & $-0.33 \star$ \\
6 OPLNIVO & -0.23 & $-0.36 \star$ \\
7 TYPB & 0.25 & - \\
8 PA & - & - \\
9 MOB & - & $0.12 \star$
\end{tabular}

$\mathrm{r}^{2}=0.35 ;$ adj. $\mathrm{r}^{2}=0.33 ; \mathrm{N}=306$

Signifikanz: ${ }^{*} \mathrm{p}<0.05 ;{ }^{* *} \mathrm{p}<0.01 ;{ }^{* * *} \mathrm{p}<0.001$

Die Interpretation der Resultate der Totalanalyse erfolgt in der Reihenfolge der signifikanten Variablen:

1 Eine geringe Beschäftigungsdauer ist vielfach gepaart mit einem hohen Schulausbildungsniveau.

Bei einem hohen Schulausbildungsniveau, beispielsweise Akademiker, erfolgt der Organisationseinstieg auf höherem Hierarchieniveau. Dieser 'Seiteneinstieg' zeigt ein etwas anderes Beschäftigungsmuster. Die 'Karrierechancen' innerhalb der Organisation sind geringer. Die für den internen Arbeitsmarkt typische 'Anwartschaft' im Rahmen einer langfristig angelegten Beschäftigung ist für die Gruppe der Personen mit hohem Ausbildungsgrad außer Kraft gesetzt (Rosenbaum, 1984).

2 Bei einem häufigen Funktionswechsel ist die Beschäftigungsdauer relativ gering.

Funktionswechsel von Arbeitnehmem vollziehen sich in der Regel in der Anfangsphase der Beschäftigungszugehörigkeit (Diekmann/Preisendörfer, 1987) zur jeweiligen Organisation. Unterschiedliche Bedingungen können hiermit verbunden sein. Dabei geht es um die Suche des Arbeitnehmers nach Stabilität innerhalb seiner Funktion im Rahmen des betrieblichen Sozialisationsprozesses. Auch Umbesetzungen aufgrund von Fehlbesetzungen sowie 'Springerfunktionen' werden durch dieses Resultat abgedeckt (Bills, 1987). Das Ergebnis bestätigt Hypothese 5 .

3 Bei einer hohen Fluktuation ist die Beschäftigungsdauer meistens gering.

Hohe Fluktuation bewirkt in der Regel eine kurze Beschäftigungszeit. Dieses Ergebnis wird indirekt bestätigt durch die Untersuchung 'Südwerk' (Diekmann/Preisendörfer, 1987). Die Autoren sehen in in einer kurzen Beschäftigungsdauer Anhaltspunkte, die gegen eine 
ausschließlich humankapitaltheoretische Begründung des Abstroms von Personal sprechen. Lorenz (1990) hebt das Interesse der Untemehmen hervor, die mit Fluktuation zusammenhängenden quasifixen Beschäftigungskosten möglichst gering zu halten.

4 Eine lange Beschäftigungsdauer ist in der Regel mit einem hohen Funktionsniveau eines Arbeitnehmers verbunden.

Es gehört zu den Kennzeichen intemer Arbeitsmärkte, daß der Aufstieg sich über eine Abfolge von unterschiedlichen, aber aufeinander bezogenen Funktionen vollzieht. Ein hohes Funktionsniveau des Arbeitnehmers sezt eine lange Beschäftigungsdauer voraus. Hier wird ein Resultat der Beförderungsanalyse bestätigt. Das Ergebnis bezieht im Gegensatz zu dem in der Hypothese 4 ausgedrückten Sachverhalt die professionellen Bürokratien mit in das Ergebnis ein.

5 Mit der Zunahme der Zahl der Befördenungen steigt die Betriebszugehörigkeitsdauer.

Beförderungen sind Ausdruck der Arbeitnehmerbindung. Sie bilden ein Kriterium für die weitere 'Karriereentwicklung' des Arbeitnehmers. Weitere Beförderungen sind vielfach an Verbleibzeiten auf bestimmten Arbeitspläızen gebunden. In der Südwerk-Studie (Diekmann/Preisendörfer, 1987) wird obiges Ergebnis relativiert. Bei einigen der neuen Mitarbeiter besteht die Tendenz, den Betrieb zu verlassen, da sie beim fïmenintemen Aufstieg ins Hintertreffen geraten sind. In einem frühen Stadium wird von Seiten der Personalleitung geprüft, inwieweit Arbeitnehmer erfolgreich bestimmte 'assessmentPositionen' (Gaertner, 1990) durchlaufen.

Kein signifikanter Zusammenhang besteht zwischen der abhängigen Variable und den Variablen Weiterbildung, Geschlecht und Organisationstyp sowie Part-Mobilität.

Bei den professionellen Bürokratien ergibt sich folgendes Bild:

Tab. 35: Beschäftigungsdaueranalyse: Professionelle Bürokratien; Pearsonkorrelation (soweit signifikant); Regressionsanalyse (TIJD als abhängige Variable)

\begin{tabular}{|c|c|c|}
\hline Variablen & $\begin{array}{l}\text { Pearson- } \\
\text { korrelation }\end{array}$ & $\begin{array}{l}\text { Regressions- } \\
\text { analyse Beta }\end{array}$ \\
\hline $\begin{array}{ll}1 & \text { PKT } \\
2 & \text { VBRL } \\
3 & \text { GBSL } \\
4 & \text { OP } \\
5 & \text { POWI } \\
6 & \text { OPLNIVO } \\
7 & \text { YOB } \\
8 & \text { PA }\end{array}$ & $\begin{array}{c}- \\
0.21 \\
-0.22 \\
- \\
-0.52 \\
-0.22 \\
- \\
-\end{array}$ & $\begin{array}{c}- \\
0.21 * * \\
-0.27 * * \\
- \\
-0.67 * * * \\
-0.24 * * \\
- \\
0.19 *\end{array}$ \\
\hline
\end{tabular}

$\mathrm{r}^{2}=0.44 ; \operatorname{adj} . \mathrm{r}^{2}=0.41 ; \mathrm{N}=151$

Signifikanz: ${ }^{*} p<0.05 ;{ }^{* *} p<0.01 ;{ }^{* * *} p<0.001$

In der Reihenfolge der signifikanten Zusammenhänge der Regressionsanalyse gilt es, die Zahlen für die professionellen Bürokratien in Worte zu fassen. 
1 Ein hoher (Grad an Funktionswechsel vollzieht sich in einer Phase, in der die Beschäflgungsdauer noch gering ist.

Dieses Ergebnis unterstreicht für die professionellen Bürokratien das Ergebnis der Totalanalyse.

2 Frauen haben in der Regel ein kürzere Beschäftigungsdauer als Männer.

In professionellen Büroksatien zeigen im Rahmen dieser explorativen Untersuchung Frauen eine geringere Beschäftigungsdauer als Männer. In professionellen Bürokratien haben Frauen im Vergleich zu Männem weniger Möglichkeiten, ein professionelles Laufbahnprofil zu entwicketn, da die Kontinuität in der Karriereentwicklung of fehlt. Biehler/Brandes (1981) sehen in innerbetrieblichen Aufstiegshürden den Gund für eine geringere Beschäftigungsdauer von Frauen gegenüber Männem.

3 Bei einem hohen Schulabschlußniveau ist die Beschäftigungsdauer in professionellen Bürokratien meistens gering.

Für die professionellen Bürokratien läßt sich das Ergebnis der Totalanalyse bestätigen.

4 Die Fluktuation in professionellen Bürokratien steigt mit zunehmender Beschäftigungsdauer.

Mit diesem Resultat kommt die in professionellen Bürokratien stärker anzutreffende Neigung der Mitarbeiter zur interorganisationalen Mobilität zum Ausdruck. Diese wird begünstigt durch die Mikrostruktur der Funktionskette, die relativ kurz ist. Beruflicher Aufstieg kann vielfach nur auf dem Weg des Betriebswechsels enreicht werden.

5 Eine längere Beschäftigungsdauer innerhalb der professionellen Bürokratien geht in der Regel einher mit Part-Mobilität. Das Überschreiten der Partgrenzen innerhalb der Organisation setzt eine bestimmte Beschäftigungsdauer voraus, bei der eine bestimmte Funktionskette durchlaufen wurde. Part-Mobilität ist vielfach auch Ausdruck des Übergangs zu einer neuen Funktionskette.

Bei dieser Analyse besteht keine signifikante Beziehung zwischen der abhängigen Variable und den Variablen Beförderung, Funktion und Weiterbildung.

Bei der Analyse der Beschäftigungszeit für Maschinenbürokratien zeichnet sich folgendes Bild ab: 
Tab. 36: Bescháftigungsdaueranalyse: Maschinenbürokratien; Pearsonkorrelation (soweit signifikant), Regressionsanalyse (TLD als abhängige Variable)

\begin{tabular}{l|c|c|} 
Variablen & $\begin{array}{l}\text { Pearson- } \\
\text { korrelation }\end{array}$ & $\begin{array}{l}\text { Regressions- } \\
\text { analyse Beta }\end{array}$ \\
\hline 1 FRT & 0.18 & $0.44 * * *$ \\
2 VERL & -0.41 & $-0.36 * * *$ \\
3 GESL & -0.27 & - \\
4 OP & -0.62 & - \\
5 FUWI & -0.26 & $-0.18 *$ \\
6 OPLNIVO & -0.17 & $-0.54 * *$ \\
7 MOB & - & - \\
8 PA & - & -
\end{tabular}

$r^{2}=0.39 ; \operatorname{adj} . r^{2}=0.36 ; N=153$

Signifikanz: ${ }^{*} p<0.05 ;{ }^{* *} p<0.01 ;{ }^{* * *} p<0.001$

Die signifikanten Zusammenhänge für die Maschinenbürokratien beinhalten folgendes in der Reihenfolge der Signifikanz:

I Ein hohes Schulausbildungsniveau der Arbeitnehmer deutet auf eine geringe Beschäftigungsdauer hin.

2 Einem hohen Funktionsniveau liegt bei Maschinenbürokratien eine lange Beschäftigungsdauer zugrunde.

Bezogen auf die Teilanalyse der Maschinenbürokratien bestätigt das Ergebnis Hypothese 4.

3 Einem hohen $\mathrm{Maß}$ an Fluktuation liegt meistens eine kurze Beschäftigungsdauer zugrunde.

Hier bestätigt sich das Ergebnis der Totalanalyse.

4 Ein hohes $\mathrm{Maß}$ an Funktionswechsel isı mit einer geringen Beschäftigungsdauer verbunden.

Kein Zusammenhang besteht zwischen der abhängigen Variable und den Variablen Beförderung (hier für die Teilstichprobe der Maschinenbürokratie), Part-Mobilität, Geschlecht und Weiterbildung.

\subsubsection{Zusammenfassung der Ergebnisse der Beschäftigungsdaueranalyse}

Im folgenden sollen die Ergebnisse der Beschäftigungsdaueranalyse zusammengefaßt werden. Betrachtet man die Gemeinsamkeiten der Einzelanalysen und strukturunabhängigen Variablen, so zeigt sich sowohl für die Maschinenbürokratien als auch die professionellen Bürokratien, daß häufig vorkommender Funktionswechsel (Bestätigung von Hypothese 5) sowie ein hohes Schulausbildungsniveau mit einer kurzen Beschäftigungsdauer gepaart sind. Im Rahmen der Totalanalyse gibt es keine Bestätigung von Hypothese 4, die 
den Zusammenhang zwischen Länge der Beschäftigungsdauer und der Höhe des Funktionsniveaus erfaßt. Im Rahmen der Teilanalyse 'Maschinenbürokratien' ergibt sich für den Zusammenhang eine Bestätigung von Hypothese 4.

Bei der spezifischen Betrachtung der Organisationskonfigurationen und den strukhurabhängigen Variablen ergibt sich das folgende Bild. Bei den professionellen Bürokratien sind Frauen gegenüber Männern aufgrund eines anderen Karriereverlaufs kürzere Zeit beschäftigt. Im Rahmen des Karriereverlaufs verbleiben Frauen auf bestimmten Funktionen, ohne daß alle Funktionen der für professionelle Bürokratien typischen Funktionsleitem durchlaufen werden.

Bei den Maschinenbürokratien entspricht ein hohes Funktionsniveau einer langen Beschäftigungsdauer, die an ein internes Durchlaufen von Funktionen gekoppelt ist. Ein solches Ergebnis läßt sich nicht auf die professionellen Bürokratien beziehen, da hierbei andere Maßstäbe was Hierarchieniveau, Funktionsanordung in Form von Funktionsketten zugrunde liegen. Funktionsketten in professionellen Bürokratien sind allgemein zugänglich für Mobilität auf unterschiedlichen Hierarchieniveaus. Der 'geschlossene Charakter' der Maschinenbürokratien fehlt bei den professionellen Bürokratien.

Während bei den Maschinenbürokratien eine hohe Fluktuation bei kurzer Beschäftigungsdauer auftritt, ist ein solcher Zusammenhang bei den professionellen Bürokratien gekoppelt an eine langfristige Beschäftigungsdauer.

Ein Schlüssel für die Erklärung könnte darin liegen, daß bei professionellen Bürokratien die Qualifikation so standardisiert ist, daß darin eine implizite Voraussetzung für Beschäftigungsstabilität gegeben ist. Obiges Resultat zeigt daneben, da $B$ man von der interorganisationalen Mobilität Gebrauch macht, um das Fehlen eines Laufbahnprofils zu kompensieren. Im Gegensatz dazu ist bei Maschinenbürokratien erst zu Beginn der Beschäftigungszeit auszuloten, welche Art und Richtung der Qualifikationsentwicklung einzuschlagen ist.

Bezieht man die empirischen Ergebnisse dieser Studie auf die Beschäftigungskriterien, die in Zusammenhang mit den Organisationskonfigurationen stehen bzw. aus der Literatur ablcitbar sind, dann zeigt sich ein weitgehend konsistentes Bild. Die empirisch ermittelten 'Beschäftigungselemente' passen in das Bild der jeweiligen Organisationskonfiguration. Bezogen auf den internen Arbeitsmarkt ergeben sich Anhaltspunkte für eine weitgehende Übereinstimmung mit den Maschinenbürokratien, während professionelle Bürokratien ebenfalls Kennzeichen interner Arbeitsmärkte zeigen, auch wenn diese weniger stark ausgeprägt sind. Diese vorläufige Einschätzung erfährt weitere Vertiefung durch Einbeziehung der empirischen Ergebnisse in Kapitel 8. 


\subsubsection{Schlußfolgerungen aus der Beschäftigungsdaueranalyse}

Die Beschäftigungszeit bzw. -dauer bzw. die Beförderungsstruktur ist Teil einer in der Realität mehr Komponenten umfassenderen Beschäftigungsstrategie (Gütermann, 1989). Gegenstand dieses Abschnittes war die gezielte Untersuchung von Beschäftigungszeit aufgrund von Funktionswechseln und deren Einflußgrößen auf Organisationskonfigurationen. Untersuchungen dieser Art beziehen sich vielfach auf einen nationalen Rahmen. Die Beschränkung der Untersuchung auf den Rahmen von vier Organisationen erlaubt nur mit Vorsicht Analogien zu nationalen Daten herzustellen. Dort, wo dies möglich war, konnten Unterschiede aber auch deutliche Übereinstimriungen festgestellt werden. Die Untersuchung eröffnet aufgrund einer weniger aggregierten Datenkonstellation die Möglichkeit, organisationsspezifische Resultate abzuleiten.

Das Ergebnis der Analyse von Beschäftigungsdauer bzw. -struktur auf intemen Arbeitsmärkten steht im deutlichen Zusammenhang mil den Maschinenbürokratien. Qua Altersaufbau, differenzierten Mobilitätsstrukturen und anderen Beschäftigungsmerkmalen unterscheiden sich diese von professionellen Bürokratien. Die Beschäftigungsverhältnisse sind in der Regel auf Dauer angelegt. Dies schlieBt auch das Risiko der Überalterung der Belegschaft ein wie es beim Produktionsuntemehmen deutlich wird. Eine langfristige Betriebszugehörigkeit dient sowohl dem Arbeitnehmer als auch dem Arbeitgeber. Sie trägt zu einer Beschäftigungsstabilität bei, die sowohl Planung als auch Kontinuität im Betriebsablauf ermöglicht.

Lang andauemde Beschäftigungsverhältnisse mit einer Beschäftigungsdauer von 10 bis 20 Jahren sind in jeder Organisation vorhanden, wenn auch unterschiedlich ausgeprägt. Dennoch zeigen die jeweiligen organisationsspezifischen Besetzungen Variationen in der Beschäftigungsdauer. Es bestehen, orientiert an der Organisationstypologie, Unterschiede. Krankenhaus und Wirtschaftsprüfungsgesellschaft als professionelle Bürokratien zeigen für die Beschäftigungsdauer deutlich voneinander abweichende Besetzungzahlen gegenüber Maschinenbürokratien. Bezieht man die Ergebnisse insbesondere die Betriebzugehörigkeitsdauer der Kategorien von 20 Jahren und länger auf die nationalen Durchschnittszahlen, dann zeigt sich, daß rund 10 Prozent der gegenwärtig Beschäftigten in den Niederlanden 20 Jahre und länger beschäftigt sind (Wolfs, 1990). Auf das Krankenhaus bezogen zeigt sich eine unterdurchschnittliche Ausprägung.

Die Wirtschaftsprüfungsgesellschaft und die Sozialversicherung liegen in etwa im nationalen Durchschnittsniveau, während das Produktionsunternehmen mit zusammen 45 Prozent deutlich über dem Durchschnitt liegt. Die nationalen Vergleichszahlen für die Bundesrepublik liegen nach Bellmann (1986) bei 20 Prozent und in den USA auch bei nur 10 Prozent.

Unter Berücksichtigung der Verteilung der übrigen Beschäftigungsdauer kann die SchluBfolgerung abgeleitet werden, daß die Beschäftigungsdauer in professionellen Bürokratien kürzer ist als in Maschinenbürokratien. Dies kann mit der stärkeren interorganisationalen Mobilität bei professionellen Organisationen erklärt werden. 


\subsection{Schlußfolgerungen}

Die Ergebnisse dieses Kapitels haben einen Teil der Komplexität gezeigt, die die Beschäftigungsformen zum Ausdruck bringen. Die hier ableitbaren Erkenntnisse beziehen sich zunächst auf die beiden Organisationskonfigurationen bzw. die Gesamtheit der untersuchten Organisationen. Erst in einem weiteren Schritt der Evaluation der Ergebnisse (Kapitel 9) soll geprüft werden, inwieweit die durch die empirische Untersuchung ermittelten Ergebnisse mit den Charakteristiken interner Arbeitsmärkte zu vergleichen sind. Wir beziehen uns daher auf die Gesamtheit der Ergebnisse dieses ersten Teils der Untersuchung.

Die infrastrukturbezogenen Analysen intemer Arbeitsmärkte werden hiermit vorläufig abgeschlossen. In den SchluBfolgerungen dieser Arbeit wird auf die theoretische Relevanz der Ergebnisse eingangen. Im nächsten Kapitel wenden wir uns der Vakanzenanalyse der vier Organisationen zu. 


\section{Die offene Stellenanalyse}

Die offene Stellenanalyse ergänzt die Infrastrukturanalyse interner Arbeitsmärkte und lenkt den Blick in zwej Richtungen der Allokation von Arbeit. Zum einen geht es um die Interaktion von intemen und externen Stellenbesetzungen. Zum anderen wird untersucht, unter welchen Bedingungen offene Stellen intem besetzt werden. Das vorliegende Kapitel glieder sich deshalb in zwei Hauptabschnitte. Gegenstand des ersten Hauptabschnittes ist die Interaktion von externer und intemer Stellenbesetzung. Dem liegt die Vorstellung zugnunde, daß die Besetzung offener Stellen selten überwiegend extern (also vom sogenannten externen Arbeitsmarkt) oder intern (d. h. im Rahmen des internen Arbeitsmarktes, also organisationsintern) geschicht (Hypothese 6). Die Prioritäten der einen oder anderen Weise hängen ab von der jeweiligen Organisationskonfiguration. Nachgegangen wird der Frage, inwieweit ein bestimmtes quantitatives Verhälmis von in- und extemen Rekrutierungen typisch ist für eine bestimmte Organisationskonfiguration.

Da die Aufmerksamkeit dem internen Geschehen des intemen Arbeitsmarktes gilt, soll im zweiten Hauptabschnitt in Form der Analyse der internen Stellenbesetzung untersucht werden, unter welchen Voraussetzungen die organisationsinterne Stellenbesetzung stattfindet und welche Prioritäten dabei eine Rolle spielen (Hypothesen 7 u. 8). Am SchluB des Kapitels soll ein Blick auf die Stellenbesetzung nach Parts geworfen werden.

Die Interaktion von extemer und intemer Stellenbesetzung ist Teil der Arbeitskräftestrategie, die die Gesamtheil der betrieblichen Strukturen, Politiken und Maßnahmen umfaßt. Ziel dabei ist es, daß Arbeitskraft und Arbeitsleistung zu akzeptablen Kosten zur Verfügung stehen (Lutz, 1987). Bestandteil der betrieblichen Arbeitsmarktstrategie ist neben der Konstifutierung des intemen Arbeitsmarktes mit den spezifischen internen Allokationsformen, die Form des Zugriffs auf den externen Arbeitsmarkt. Die Vorteile sieht Lutz zum einen in der Nutzung der auf dem externen Arbeitsmarkt bestehenden Konkurrenz zwischen Arbeitsanbietem und Arbeitskraft und zum anderen in der Möglichkeit strategischer Umorientierung in Form der Externalisierung von Funktionen und Problemlösungen.

Der folgende Abschnitt greift einen Teilaspekt der Arbeitskräftestrategie auf, es geht um die empirische (und damit auch quantitative) Bestimmung der Interaktion von ex- und interner Stellenbesetzung. Bevor im einzelnen darauf eingegangen wird, gilt es, kurz die Variablen vorzustellen, die in die Untersuchung einbezogen wurden. 


\subsection{Interaktion zwischen ex- und interner Stellenbesetzung}

Im Mittelpunkt dieses Abschnitts steht neben der Einführung des Datenmaterials die Analyse der Interaktion von interner und extemer Stellenbesetzung (Hypothese 6).

\subsubsection{Beschreibung der Variablen der offenen Stellenanalyse}

Mit der Einführung der Variablen soll ein erster Überblick über die Beschaffenheit der Variablen in Bezug auf Häufigkeitsverteilung bzw. der Ausprägung der Variablen gegeben werden.

\subsubsection{Die abhängigen Variablen der offenen Stellenanalyse}

Die offene Stellenanalyse wird durch zwei abhängige Variablen charakterisiert, die eine steht für die Interaktion von in- und extemer Stellenbesetzung. Die andere für die ausschließlich interne Reallokation. Die erste soll zunächst erläutert werden.

Als Maßstab für interne und/oder externe Besetzung offener Stellen wurde die Variable 'VERVUL' geschaffen. Sie kann zwei Werte annehmen. Wert 1 repräsentiert die Stellenbesetzung durch Rekrutierung von Personal vom organisationsinternen Arbeitsmarkt. Wert 2 charakterisiert die Besetzung der offenen Stelle vom extemen Arbeitsmarkt (Schwan/ Soeters, 1991a).

Die folgende Tabelle zeigt in einer ersten Übersicht für die Organisationen die Besetzung offener Stellen aus internen und extemen Rekrutierungsquellen.

Tab. 37: Offene Stellenbesetzung in vier Organisationen im Dreijahreszeitraum 1986-1988

\begin{tabular}{|c|c|c|c|}
\hline & Art der stellen & beset zung & \\
\hline $\begin{array}{l}0 \\
r \\
g .\end{array}$ & 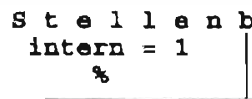 & $\begin{array}{c}\text { a } \operatorname{etz} \mathrm{z} \text { u } \\
\text { extern }=2 \\
\%\end{array}$ & total $_{\%}$ \\
\hline $\mathbf{K}$ & $137(36)$ & $244(64)$ & $381(100)$ \\
\hline w & $11(24)$ & $34(76)$ & $45(100)$ \\
\hline $\mathbf{s}$ & $170(66)$ & $87(34)$ & $257(100)$ \\
\hline $\mathbf{P}$ & $217 \quad(78)$ & $62(22)$ & $279(100)$ \\
\hline t & $535 \quad(56)$ & $427(44)$ & $962(100)$ \\
\hline
\end{tabular}

Erläuterung: Die Prozentwert befinden sich in Klammem.

$\mathrm{K}=$ Krankenhaus; $\mathrm{W}=\mathrm{Wirtsch}$ aftsprüfungsuntemehmen; $\mathrm{S}=$ Sozialversicherung; $\mathrm{P}=$ Produktionsuntemehmen; $\mathrm{t}=$ total 
Aus der Tabelle läßt sich ableiten, daß Stellenbesetzungen bei den untersuchten Organisationen in allen Fällen intern als auch extem erfolgen. Im Verhältnis der ex-bzw. intemen Stellenbesetzung zeichnen sich für die hier behandelten Organisationskonfigurationen deutliche Stellenbesetzungsmuster ab.

Bei der Betrachtung der Stellenbesetzung fällt auf, daß bei professionellen Bürokratien eine Präferenz für externe Rekrutierung von Personal vorherrscht. Von der Tendenz bestätigt sich die aus der Infrastrukturanalyse abgeleitete Erkenntnis, daß Maschinenbürokratien mehr interne Dynamik hinsichtlich Mobilität aufweisen. Bei Sozialversicherung und Produktionsuntemehmen dominiert die inteme Stellenbesetzung, während die Zahl der 'extemen' Stellenbesetzungen relativ schwach ausgeprägt ist.

Bei den professionellen Bürokratien ist dies umgekehrt. Umfang und das Verhältnis der Rekrutierungen im einen oder anderen Fall kennzeichnen die für die Organisationskonfiguration typische Interaktion zwischen interner und externer Stellenbesetzung.

Im Vorfeld der Analyse intemer Stellenbesetzungen soll hier die abhängige Variable der Analyse vorgestellt werden. Dabei handelt es sich um eine Variable, bei der von einer möglichen Priorität intemer Stellenbesetzungen aus naheliegenden organisatorischen und beschäftigungspolitischen Einheiten ausgegangen wird. Die interne Stellenbesetzung hängt von einer Vielfalt organisationsstruktureller Grenzen ab, die die interne Mobilitätsdynamik mitbestimmen (Scott, 1987; Gunz, 1989). Als 'Einheit' in diesem Sinne kommt die Funktionskette, die Abteilung, aber auch die Berufsgruppe in Frage. Unter Heranziehung 'üblicher Besetzungsmuster' läßt sich eine Prioritätenfolge (von Priorität 1 bis Priorität 8 ) interner Besetzungen ableiten (Hypothesen 7 u. 8).

Die abhängige Variable (DIP) repräsentiert die Verknüpfung der unterschiedlichen Prioritäten intemer Stellenbesetzung. Die höchste Priorität der Suche nach geeigneten Kandidaten findet in nächster Umgebung statt, d. h. in derselben Abteilung und derselben Funktionskelte sowie derselben Berufsgruppe. Die geringste Priorität umfaßt ein breites Suchfeld, das sich auf die gesamte Organisation bezieht. Die Variable deckt ein Spektrum ab von der höchsten Priorität, nämlich der Reknutierung aus unmittelbarem Umkreis bisheriger Beschäftigung bis hin zur geringsten Priorität der organisationsweiten Suche nach geeigneten Kandidaten. Zwischen der höchsten und der geringsten Priorität liegen in abnehmender Prioritätenfolge Kombinationen aus Abteilung, Funktionskette und Berufsgruppe als inteme 'Rekrutierungsfelder' (Vietorisz/Harrison, 1978). 
Tab. 38: Interne Stellenbesetzung: Bedeutung der abhängigen Variable (DP)

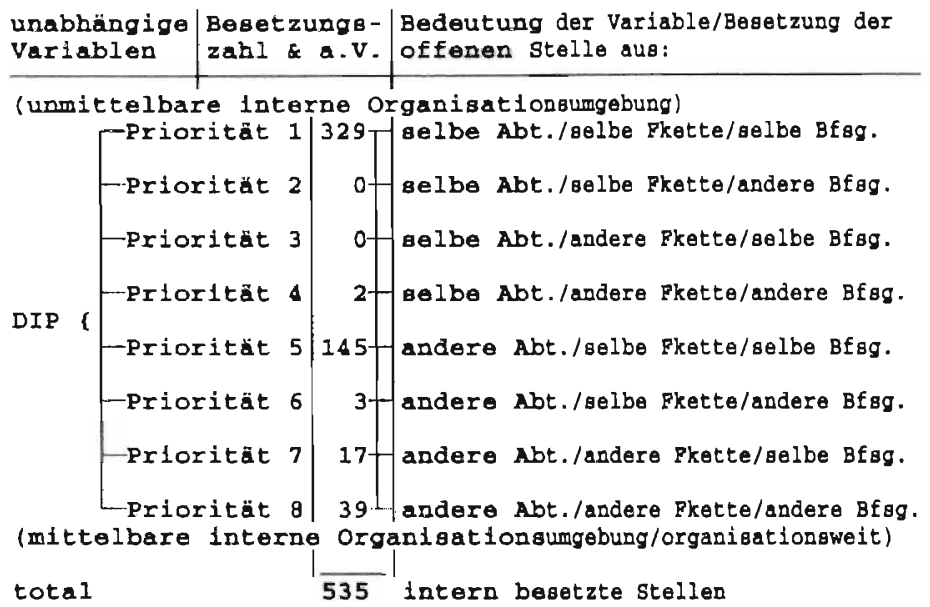

Erläuterung der Abkürzungen: Abt.=Abteilung; Fkette=Funktionskette; Bfsg.=Berufsgruppe; a. V.=abhängige Variable (DIP)

Die Variable 'DIP' (DIP=Variable 1-8) verknüpft die in der Abbildung genannten acht Prioritäten in der Analyse zu einer Variable. Die höchste Prionität (Priorität 1) der Variable 'DIP' bedeutet interne Stellenbesetzung aus der nächsten Organisationsumgebung bisheriger Beschäftigung. Die geringste Priorität (Priorität 8) symbolisiert eine Stellenbesetzung, bei der die Suche organisationsweit erfolgt.

Berücksichtigt wurden alle Prioritäten, die mit Zahlenmaterial auszufüllen waren. Alle übrigen Prioritäten wurden im Rahmen der spezifischen Berechnung gleich null gesetzt, um dem jeweiligen Kodierungseffekt Geltung zu verschaffen. Bei der intemen Besetzung von Stellen besteht eine Präferenz der Reknutierung aus dem unmittelbaren Organisationsumfeld bisheriger Beschäftigung mit 340 Rekrutierungen. Von der Häufigkeit der Besetzung folgt Prioritä 5 mit 145 Rekrutierungen. Der Rest verteilt sich auf die übrigen Prioritäten.

\subsubsection{Die unabhängigen Variablen der offenen Stellenanalyse}

Die folgenden Variablen sind als unabhängige Variablen in die Regressionsanalyse eingegangen. Hier gilt es, die Variablen mit spezifischer deskriptiver Statistik zu unterbauen.

1 Die Variable 'FKT' (Funktion) entspricht der der Mobilitätsanalyse. Funktion charakterisiert grob die Tätigkeit, die Arbeitnehmer innehaben. Sämtliche erfaßten Funktionen wurden nach einem Hierarchiestufenmodell des Niederländischen Lohntechnischen Dienstes (1987) durch Bewertung einer Skala von acht Hierarchiestufen zugeordnet. Die Zahl 1 umschreibt das unterste Hierarchieniveau, die Zahl 8 das höchste Hierachieniveau. 
Die folgende Tabelle gibt einen Einblick in die Funktionsniveauverteilung, Dabei wird wieder unterschieden zwischen interner und extemer Stellenbesetzung,

Tab. 39: Funktionsverteilung der 20 besetzenden offenen Srellen im Dreijahreszeitraum 1986-1988

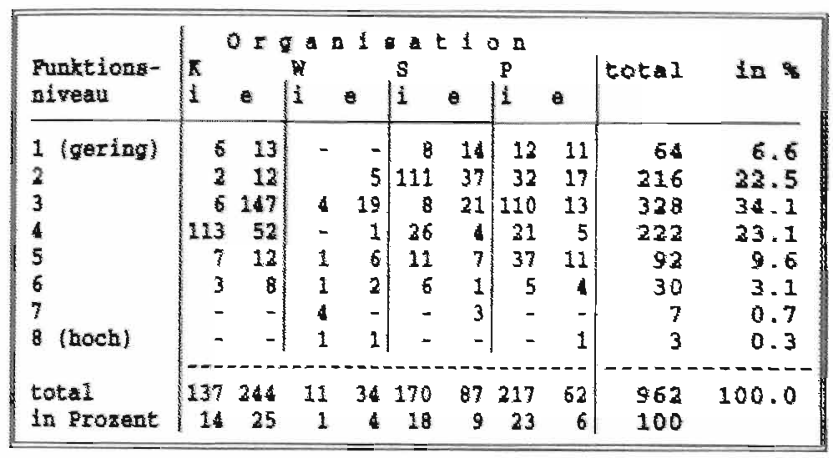

Erklärung: $K=$ Krankenhaus; $S=$ Sozialversicherung; $W=W i r$ schaftsprifungsuntemehmen; P=Produktionsunternehmen; i=intern; $e=$ extem

Die Tabelle zeigt die Verteilung der Funktionen unter Berücksichtigung der jeweiligen Rekrutierungsquelle. Aussagen über die 'ports of entry' lassen sich hier nur begrenzt anführen, da die Funktionsniveaus der eintretenden Mitarbeiter hier zum Zwecke der Vergleichbarkeit stark konzentriert sind. Aus Gründen der Vergleichbarkeit sind die Funktionen in der Realität einer viel differenzierteren Funktionshierarchie zuzurechnen. Unter diesem Vorbehalt ergibt sich aus der Tabelle folgendes Bild.

Im Fall des Krankenhauses bildet Funktionsniveau 3 hauptsächlich die 'ports of entry' mit 147 externen Einstellungen. Quantitativ umfangreicher ist Funktionsniveau 4 mit insgesamt 165 Rekrutierungen. Hierbei entfallen 113 auf interne Rekrutierungen. Ein Teil dieser Rekrutienungen repräsentier dabei die Übemahme von Personal nach erfolgter Ausbildung. Bei der Wirtschaftsprüfungsgesellschaft verzeichnet Funktionsniveau 3 mit 19 externen Einstellungen die höchste Besetzungsrate mit extern rekrutiertem Personal. Funktionsniveau 5 des Krankenhauses ist gekennzeichnet durch die Präferenz in- und extemer Stellenbesetzung ( 7 inteme gegenüber 12 extemen Rekrutierungen auf diesem Niveau).

Bei den Maschinenbürokratien liegt der Schwerpunkt auf der internen Stellenbesetzung. Die exteme Stellenbesetzung wird dadurch zur Restkategorie. Bei der Sozialversicherung liegt unter diesen Bedingungen der Schwerpunkt der intemen Rekrutierung bei Funktionsniveau 2 mit 111 internen Rekrutierungen. Auch die Zahl der externen Rekrutierung erreicht auf Funktionsniveau 2 mit 37 Einstellungen das höchste Niveau. Beim Produktionsunternehmen liegt eine Verteilung zugrunde, die auf die unteren Funktionsniveaus konzentrier ist. So beläuft sich die Zahl der intemen Rekrutierungen auf 110 auf dem Funktionsniveau 3 und 17 externe Einstellungen auf Funktionsniveau 2. Auf dem höchsten Funktionsniveau der Sozialversicherung sind keine externen Stellenbesetzungen vor- 
genommen worden. Beim Produktionsunternehmen trifft dies in einem Fall zu. Bei beiden Organisationen haben sich auf den höheren Funktionsniveaus, abgesehen von der einen Ausnahme, im Beobachtungszeitraum von drei Jahren keine offenen Stellen ergeben. Tabelle 39 bietet Anhaltspunkte für die Bestätigung von Hypothese 2.

2 Die Variable 'Typ' (TYPE) differenziert zwischen professioneller Bürokratie und Maschinenbürokratie. Wert 1 steht für die 'professionelle Bürokratie', Wert 2 für die Maschinenbürokratie.

3 Aufschluß über die Gründe für das Entstehen der offenen Stelle gibt die Variable 'ONTVAC'. Die unterschiedlichen Gründe wurden dichotomisiert. Der Wert 1 repräsentiert die Entstehung "neuer Arbeitsplätze" durch Wachstum oder technologische Entwicklung. Wert 2 faßt Gründe zusammen, die auf einen 'intemen' Entstehungsgrund hinweisen, dazu zählt intraorganisationale Personalbewegung in Form von Versetzungen, Beförderungen und allgemeiner Reorganisation, Kündigungen sowie das Ausscheiden aufgrund des Erreichens der Altersgrenze. Die folgende tabellarische Übersicht gibt nicht nur Aufschluß über die Verteilung der Werte bei den verschiedenen Organisationen sondem ermöglicht auch eine Einschätzung in Bezug auf in- oder exteme Stellenbesetzung.

Tab. 40: Verteilung der Entstehungsgründe offener Stellen und deren Besetzung im Dreijahreszeitraum

\begin{tabular}{|c|c|c|c|c|c|c|c|c|c|c|}
\hline $\begin{array}{l}\text { Entetehungs- } \\
\text { grunde off. } \\
\text { stellen }\end{array}$ & $\begin{array}{l}\mathbf{R} \\
1\end{array}$ & $\begin{array}{l}0 r \\
0\end{array}$ & $w^{a} n$ & $\begin{array}{l}18 \\
\theta\end{array}$ & $\begin{array}{l}\mathrm{s} \\
\mathbf{i}\end{array}$ & 10 & $\stackrel{O n}{P}_{i}$ & e & total & $10 \%$ \\
\hline $\begin{array}{l}1 \text { neue Ar- } \\
\text { beitoplatze }\end{array}$ & 11 & & - & 27 & - & 74 & 1 & 61 & 174 & 18.1 \\
\hline $\begin{array}{l}2 \text { interne } \\
\text { Personalbe- } \\
\text { wegung }\end{array}$ & 126 & 244 & 11 & 7 & 170 & 13 & 216 & 1 & 788 & 81.9 \\
\hline $\begin{array}{l}\text { total } \\
\text { in Prozent }\end{array}$ & $\begin{array}{r}137 \\
14\end{array}$ & $\begin{array}{r}244 \\
25\end{array}$ & $\begin{array}{r}11 \\
1\end{array}$ & 34 & $\begin{array}{r}170 \\
18\end{array}$ & $\begin{array}{r}87 \\
9\end{array}$ & $\begin{array}{r}217 \\
23\end{array}$ & $\begin{array}{r}62 \\
6\end{array}$ & $\begin{array}{l}962 \\
100\end{array}$ & 100.0 \\
\hline
\end{tabular}

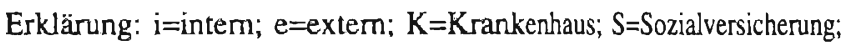
$\mathrm{W}=$ Wirtschaftsprüfungsuntemehmen; $\mathrm{P}=$ Produktionsunternehmen;

Aus der Tabelle lassen sich folgende 'Stellenbesetzungsmuster' ableiten: Zunächst wird deutlich, daß neue Arbcitsplätze (Ausprägung 1) überwiegend extem mit Personal besetzt werden. Dies gilt mit Ausnahme des Krankenhauses für alle der hier untersuchten Organisationen. Die Besetzung neu entstandener Arbeitsplätze aus intemen Rekrutierungsquellen ist beim Krankenhaus mit 11 Fällen stark und im Produktionsunternehmen mit einem Fall schwach ausgeprägt. Die Besetzungspräferenz aus intemen Rekrutierungsquellen bei neu entstandenen Arbeitsplätzen im Krankenhaus dürfte mit den professionellen Qualifikationsstandards zusammenhängen. Von den elf intern besetzten neuen Arbeitsplätzen sind neun Arbeitsplätze einem professionellen Qualifikationsstandard zuzuschreiben, zwei entsprechen einer Jedermann-Qualifikation, die intern 'on the job' erworten wird. Exterme 
Rekrutienungen konzentrieren sich vorrangig auf Personen, die noch keinen professionellen Berufsstatus haben. Erst die professionelle Ausbildung führt zu einer 'übertragbaren' professionellen Qualifikation.

Die inteme Personalbewegung (Ausprägung 2) berücksichtigt die Entstehung freiwerdender Arbeitspläıze aufgrund intemer Mobilität. Mit Ausnahme des Krankenhauses dominitrr hier erwartungsgemäß die interne Stellenbesetzung gegenüber der externen. Die Präferenz der intemen Stellenbesetzung aufgnund eines hohen Personalumsatzes ist sowohl bei professionellen Bürokratien als auch Maschinenbürokratien deutlich entwickelt.

Während die exteme Rekrutierung in den anderen Organisationen auf Ausnahmen beschränkt bleibt, ist sie beim Krankenhaus stark ausgeprägt. Erklären läßt sich dies mit einem relativ hohen Anteil von Auszubildenden, die vom extemen Arbeitsmarkı rekrutiert werden.

Die im folgenden vorzustellenden Variablen sind Gegenstand der Analyse intemer Stellenbesetzung. Sie werden ausschließlich für diesen Zweck gebraucht. Ihrer Struktur nach entsprechen sie Kriterien organisationsintemer Stellenbesetzung.

4 Die Variable 'Fluktuation' entspricht der Variable der Mobilitätsanalyse. Fluktuation - VERL) amselireibt die durchschnittliche Fluktuation per Organisation.

5 Die Variable 'Funktionskette' (KETEN) gehört zur Gruppe der Variablen, die die Zahl potentieller Kandidaten aus derselben Funktionskette für eine interne Stellenbesetzung charakterisieren. Verknüpt werden hier zwei Variablen, die die Stellenbesetzung aus gleicher Funktionskette umschreiben innerhalb und außerhalb der betroffenen Abteilung. (Hypothese 7)

6 Die Variable 'selbe Berufsgruppe' (ZELBER) bezieht sich auf die Zahl der potentiellen Kandidaten aus derselben Berufsgruppe, die für eine interne Stellenbesetzung in Frage kommen. Sie verknüpft die Anzahl der intemen Stellenbesetzungen aus der gleichen Berufsgnuppe innerhalb und außerhalb der betroffenen Abteilung. Aufgrund von Multikollinearität konnte diese Variable bei einem Teil der Analysen nicht berücksichtigt werden. (Hypothese 8)

7 'Andere Benufsgruppe' (ANBER) verknüpft als Variable die Zahl der potentiellen intemen Kandidaten für Stellenbesetzungen aus anderen Berufsgruppen innerhalb und außerhalb der betroffenen Abteilung. Erfaßt wurden dabei alle Kandidaten, die innerhalb der Organisation potentiell in Frage kamen für die Besetzung, soweit sie vom Gehaltsniveau im Gehaltsbereich der offenen Stelle lagen.

Die drei Variablen KETEN, ZELBER und ANBER erfassen Wirklichkeitsmerkmale. Die einzelnen Merkmalsausprägungen werden von den Variablenwerten erfaßt. Die durch Messung gewonnenen Daten sind nominalskaliert.

8 Desweiteren wird hier die Variable 'Bruttomonatsgehalt' (SAL) eingeführt. Das Erkenntnisinteresse konzentriert sich auf den Zusammenhang zwischen offenen Stellen und 
Entlohnung. Zugrunde liegt ein mittleres Bruttomonatsgehalt des potentiellen Besetzers der Stelle im Rahmen des funktionsbezogenen Gehaltsspektrums. Die sogenannten Stellenbesetzungsvariablen finden sich in der folgenden Werteübersicht wieder.

\subsubsection{Werteübersicht der Variablen der offenen Stellenanalyse}

Die Werteübersicht der Variablen der offenen Stellenanalyse besteht aus drei Einzelanalysen. Dabei handelt es sich um die allgemeine Werteübersicht, gefolgt von der Varianzanalyse und den Pearsonkorrelationen.

Im folgenden schließt sich die allgemeine Werteübersicht der hier vorgestellten Variablen an. Beschrieben werden Mittelwerte, Standardabweichung, Minimum und Maximum sowie die Schiefheit der Verteilung (Skewness). Die Anzahl der Fälle beläuft sich auf insgesamt 962. Die Definition der Werte erfolgt zum Teil in Abschnitt 5.3. Ansonsten handelt es sich um Standarddefinitionen im Rahmen der SPSS-Software.

Tab. 41: Werteübersicht der offenen Stellenanalyse

\begin{tabular}{|c|c|c|c|c|c|}
\hline $\begin{array}{l}\text { Variablen } \\
\text { (niedrig=) }\end{array}$ & Mittelwert & $\begin{array}{l}\text { Standard- } \\
\text { abweichung }\end{array}$ & Minimum & Maxtmum & skewn. \\
\hline $\begin{array}{l}1 \text { verval } \\
\text { (Intern begetzt) }\end{array}$ & 1.4 & 0.5 & 1 & 2 & 0.08 \\
\hline $\begin{array}{l}2 \text { fkt (nledrlge } \\
\text { Funktion) }\end{array}$ & 3.2 & 1.5 & 1 & 8 & 0.52 \\
\hline $\begin{array}{l}3 \text { ontrac (neue } \\
\text { arbettoplatze) }\end{array}$ & 1.8 & 0.4 & 1 & 2 & 0.08 \\
\hline $\begin{array}{l}\text { verl } \\
\text { (nied. Flukt.) }\end{array}$ & 5.4 & 1.1 & 3.6 & 6.5 & -0.79 \\
\hline $\begin{array}{l}5 \text { type (prof. } \\
\text { Bürokratie) }\end{array}$ & 1.5 & 0.5 & 1 & 2 & 0.00 \\
\hline $\begin{array}{l}6 \text { sal (Brutto- } \\
\text { monategehalt)" }\end{array}$ & 2846.1 & 1154.6 & 1140 & 9998 & 1.55 \\
\hline
\end{tabular}

$\mathrm{N}=962 ;{ }^{*}=$ in Gulden; Skewn. = Skewness

Auf eine Besprechung der Tabelle wird aufgrund der ausführlichen Vorstellung der Variablen und der Verbalisierung der Resultate verzichtet. Diese Werteübersicht der Variablen wird ergänzt durch Variablen der intemen Stellenbesetzung. Die folgende Tabelle ermöglicht eine erstes Verständnis der Werte. Die Werteibbersicht sieht wie folgt aus: 
Tab. 42: Werteübersicht der internen Stellenbesetzung

\begin{tabular}{|c|c|c|c|c|c|}
\hline $\begin{array}{l}\text { Variablen } \\
\text { (nledrlg=) }\end{array}$ & Mittelwert & \begin{tabular}{|l} 
Standard- \\
abweichung
\end{tabular} & Minimum | & Maximum & Skewn. \\
\hline $\begin{array}{l}1 \text { dip ( Besetzung } \\
\text { aus selbe Abt.. } \\
\text { selbe Fktk.) } \\
(\mathrm{N}=535)\end{array}$ & 2.8 & 2.4 & 1 & 8 & 0.81 \\
\hline $\begin{array}{l}2 \text { ketent } \\
\text { (p. B. : wenig aus } \\
\text { Pktk.) } \\
(\mathrm{N}=527)\end{array}$ & 24.0 & 22.5 & 1 & 120 & 1.66 \\
\hline $\begin{array}{l}3 \text { zelbert } \\
\text { (p. B.: wenig aus } \\
\text { sel. Beruf ggrup. }) \\
(N=527)\end{array}$ & 20.7 & 23.5 & 1 & 176 & 1.96 \\
\hline $\begin{array}{l}4 \text { anbert } \\
\text { (D. B. : wenig aus } \\
\text { and. Beruf sgrup.) } \\
(\mathrm{N}=535)\end{array}$ & 9.9 & 15.8 & 0 & 176 & 3.87 \\
\hline
\end{tabular}

Erläuterung: Abt.=Abteilung; and.= andere; Skewn. = Skewness; Fktk.=Funktionskette; Benfsgrup. $=$ Berufsgruppe; . B. $=$ potentielle Besetzung; + gilt nur für offene Stellen, die intem besetzt wurden.

Die zweite Einzelanalyse ist die Varianzanalyse. Sie zeigt auf der Basis von Mittelwerten wie welche Variable besetzt ist. Im einzelnen ergeben sich für die jeweilige Organisation die folgenden Perspektiven. Auf eine Interpretation dieser beschreibenden Statistik soll hier verzichtet werden zugunsten der Interpretation einer höheren statistischen Anwendungsstufe.

Tab. 43: Varianzanalyse der Besetzung offener Stellen

\begin{tabular}{|c|c|c|c|c|c|c|}
\hline \multirow[t]{2}{*}{$\begin{array}{l}\text { Variablen } \\
\text { (niedrig=) }\end{array}$} & \multicolumn{6}{|c|}{ 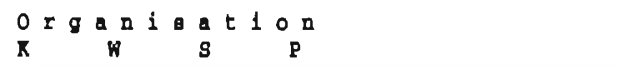 } \\
\hline & \multicolumn{4}{|c|}{ M t t e l werte } & F & slgaif. \\
\hline $\begin{array}{l}1 \text { vervul } \\
\text { (intern besetzt) }\end{array}$ & 1.6 & 1.8 & 1.3 & 1.2 & 35.1 & $\omega *$ \\
\hline $\begin{array}{l}2 \text { ontrac (neue } \\
\text { Arbeit Bplatze) }\end{array}$ & 2.0 & 1.4 & 1.7 & 1.8 & 52.5 & $\# *$ \\
\hline $\begin{array}{l}3 \text { fkt (niedrige } \\
\text { Punktion) }\end{array}$ & 3.5 & 4.0 & 2.6 & 3.2 & 33.5 & $\star \star \star$ \\
\hline $\begin{array}{l}\text { 4 verl } \\
\text { (nledrige Flukt.) }\end{array}$ & 6.5 & 5.5 & 6.0 & 3.6 & - & - \\
\hline $\begin{array}{l}5 \text { type } \\
\text { (prof. Burokrat, ) }\end{array}$ & 1.0 & 1.0 & 2.0 & 2.0 & - & - \\
\hline $\begin{array}{l}6 \text { sal (Brutto- } \\
\text { monatsgehalt) }\end{array}$ & 2234.0 & 145.2 & 3063.3 & 3272.0 & 90 & $\pitchfork \star$ \\
\hline
\end{tabular}

$\mathrm{N}=962 ;{ }^{*}$ = in Gulden; -=keine Ausprägung, da organisationsspezifische

Werte; Signifikanz: *** $=p<0.001$ 
Für die interne Stellenbesetzungsanalyse sind die folgenden organisationsspezifischen Mittelwerte neben anderen Werten zu verzeichnen:

Tab. 44: Varianzanalyse der Stellenbesetzung

\begin{tabular}{|c|c|c|c|c|c|c|}
\hline \multirow{2}{*}{$\begin{array}{l}\text { Variablen } \\
\text { (niedrig=) } \\
\text { Integrations- } \\
\text { variablen }\end{array}$} & \multicolumn{6}{|c|}{ 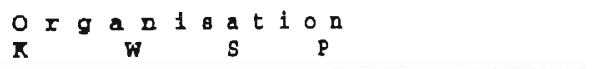 } \\
\hline & $M i t$ & e 1 & Ite & & F & slgnif. \\
\hline $\begin{array}{l}1 \text { dip (Besetzung } \\
\text { aug selbe Abt.; } \\
\text { gelbo Fktk. } \\
(N=535)\end{array}$ & 4.9 & 4.9 & 2.7 & 1.5 & 88.2 & 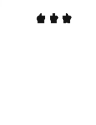 \\
\hline $\begin{array}{l}2 \text { keten+ } \\
\text { (wenig aus Funk- } \\
\text { tionskette) } \\
(\mathrm{N}=527 \text { ) }\end{array}$ & 35.2 & 18.9 & 36.1 & 8.2 & 98.0 & $t \pm t$ \\
\hline $\begin{array}{l}3 \text { zelbert } \\
\text { (wenlg aus der } \\
\text { gelb. Beruf Bgrup. ) } \\
(N=527 \text { ) }\end{array}$ & 42.9 & 18.9 & 24.1 & 5.2 & 117.2 & $\| * *$ \\
\hline $\begin{array}{l}\text { anbert } \\
\text { (wentg aus ander- } \\
\text { or Berufegruppe) } \\
(\mathrm{N}=535)\end{array}$ & 3.0 & 0.4 & 23.8 & 4.0 & 99.2 & 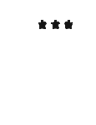 \\
\hline
\end{tabular}

Erläuterung: Abt.=Abteilung; and.= andere; Fktk.=Funktionskette; $\mathrm{K}=\mathrm{Krankenhaus;}$ $\mathrm{W}=$ Wirtschaftsprüfungsuntemehmen; $S=$ Sozialversicherung; $P=$ Produktionsunternehmen; $\mathrm{F}=$ Testgröße;+ gilt nur für offene Stellen, die intern besetzt wurden; Signifikanz: ${ }^{* * *}=$ $\mathrm{p}<0.001$

Wie schon anfangs erwähnt, besteht die zweite Einzelanalyse u. a. aus den Pearsonkorrelationen. Sie vermitteln einen ersten Eindruck über signifikante bzw. nicht signifikante Zusammenhänge zwischen zwei Variablen. Die Übersicht der Zusammenhänge befindet sich im Anhang (Tab. 45-47). Auf die Erläuterung der signifikanten Zusammenhänge wird hier verzichtet, da die Pearsonkorrelationen für Vergleichszwecke bei den Regressionsanalysen herangezogen werden.

Die quantitativen Grundlagen bieten die Ausgangssituation für die weiteren Analysen. Bei allen Regressionsanalysen wurde der Wert der Konstante in die Berechnungen einbezogen. Aus Gründen der Übersichtlichkeit wurde darauf verzichtet, diese im einzelnen in die Tabellen zu integrieren. Bei den Analysen besteht ein geringes quantitatives Übergewicht der Maschinenbürokratien. Die Ergebnisse können von diesem Übergewicht beeinfluBt sein. Im Vordergrund steht zunächst die offene Stellenanalyse.

\subsubsection{Die Interaktion der Besetzung offener Stellen aus ex- und intemen Quellen}

Die hier angesprochene Form der Stellenbesetzung richtet sich auf das Überschreiten der äußeren Organisationsgrenzen im Falle der extemen Rekrutierung. Für die inteme Rekn- 
tierung gilt, daß der potentielle Kandidat innerorganisatorische Grenzen (Position in der Funktionskette; Abteilungsgrenzen) überwinden muB (Scott, 1987).

Die folgenden Analysen basieren auf der vereinfachenden Annahme, daB die Rekrutierungsentscheidung sich entweder auf in- oder externe Kandidaten bezieht (Hypothese 6). Von der ökonomischen Bedeutung spielt die Organisationsgrenze nach außen eine entscheidende Rolle (Reve, 1990). Der Grad an Durchlässigkeit für Personen von außen bestimmt den Umfang der Offenheit bzw. Geschlossenheit der Organisation. Inteme Arbeitsmärkte lassen sich durch ein hohes $\mathrm{MaB}$ an Geschlossenheit charakterisieren. Die Geschlossenheit läßt sich messen durch die Verteilung der 'ports of entry' sowie durch die Rekrutierungsmuster, ausgedrückt durch das Verhältnis von in- bzw. extemen Rekrutierungen. Den äußeren Organisationsgrenzen kommt eine Filterfunktion in Bezug auf diejenigen zu, die unter bestimmten Voraussetzungen Zugang zur Organisation finden (Feijen, 1991). In diesem Abschnitt soll anhand der Resultate der Frage nachgegangen werden, unter welchen Bedingungen intern oder extern rekrutiert wird.

Der folgende Analyseschritt besteht aus der Ubemahme der signifikanten Pearsonkorrelationen (Anlage, Tab. 45-47) für die bei dieser Analyse benutzen Variablen (die jeweiligen Variablen korreliert mit der "vervul"-Variable). Wie bei der Mobilitätsanalyse wird auch hier neben der Totalanalyse die Differenzierung in Subgruppen vorgenommen mit der bekannten Differenzierung nach Organisationskonfigurationen. Die folgende Tabelle ermöglicht eine Übersicht über die Ergebnisse der Regressionsanalyse.

Tab. 48: Besetzung offener Stellen: total; Pearsonkorrelation; Regressionsanalyse (vervul als abhängige Variable)

\begin{tabular}{|c|c|c|}
\hline Variablen & $\begin{array}{l}\text { Pearson- } \\
\text { korrelation }\end{array}$ & $\begin{array}{l}\text { Regressions- } \\
\text { analyse Beta }\end{array}$ \\
\hline $\begin{array}{ll}1 & \text { PKT } \\
2 & \text { VERL } \\
3 & \text { ONTVAC } \\
4 & \text { TYPB }\end{array}$ & $\begin{array}{r}-0.05 \\
0.30 \\
-0.89 \\
-0.37\end{array}$ & $\begin{array}{c}- \\
0.04 * \\
-0.86 * \star \\
0.04 *\end{array}$ \\
\hline
\end{tabular}

$\mathrm{r}^{2}=0.79 ;$ adj. $\mathrm{r}^{2}=0.77 ; \mathrm{N}=962$

Signifikanz: ${ }^{*} p<0.05 ;{ }^{* *} p<0.01 ;{ }^{* * *} p<0.001$

Die Regressionsberechnung zeigt für die Totalanalyse die folgenden signifikanten $\mathrm{Zu}-$ sammenhänge in der Reihenfolge der Signifikanz:

1. Bei einem hohen $\mathrm{Maß}$ an intemen personellen Veränderungen, erfolgt die Stellenbesetzung vorwiegend intern.

Die äußere Organisationsgrenze markiert die Geschlossenheit der Organisation und unterstreicht damit den Charakter interner Arbeitsmärkte. Außerdem geht von der äußeren Grenze eine Schutzfunktion aus, die einerseits von der Tendenz den Insider gegenüber dem Outsider bevorteilt (Lindbeck/Snower, 1988) und andererseits den 'stategic core', d. 
h. die 'assets of high specifity' schützt im Sinne des Erreichens der strategischen Ziele der Unternehmung (Reve, 1990).

2 Mit einem hohen Fluktuationsgrad dominiert die externe Stellenbesetzung gegenüber der internen Stellenbesetzung.

Hohe Fluktuation wirkt der Institutionalisierung von Mustern der Stellenbesetzung entgegen. Das Interesse an einer 'schnellen' Wiederbesetzung der offenen Stelle verstärkt die Neigung der Entscheidungsträger, nach externen Kandidaten Ausschau zu halten.

3 Das Resultat, das die regressionsanalytische Beziehung zwischen 'type' und 'vervul' ausdrückt, ist nicht als zuverlässiges empirisches Ergebnis zu betrachten. Es handelt sich um ein analytisch und rechentechnisches Artefakt.

Keine Beziehung besteht zwischen der abhängigen Variable und der unabhängigen Variable 'Funktion'.

Ergänzend wurde unter Benutzung des obigen Datensatzes eine Diskriminantenanalyse (siehe Anlage, Tab, 49) durchgeführt. Die standardisierten Diskriminantenkoeffizienten bestätigen das Ergebnis der Regressionsanalyse analysenspezifisch.

Bei den professionellen Bürokratien ergibt sich folgendes Bild signifikanter Zusammenhänge:

Tab. 50: Besetzung offener Stellen: prof. Bürokratien Pearsonkorrelation; Regressionsanalyse (vervul als abhängige Variable)

\begin{tabular}{ll|l|l|} 
Variablen & $\begin{array}{l}\text { Pearson- } \\
\text { korrelation }\end{array}$ & $\begin{array}{l}\text { Regressions- } \\
\text { analyse Beta }\end{array}$ \\
\cline { 2 - 3 } 1 & FKT & - & $-0.06 *$ \\
2 & VERL & - & $-0.09 *$ \\
3 & ONTVAC & -0.26 & $-0.78 *$
\end{tabular}

$r^{2}=0.54 ;$ adj. $r^{2}=0.53 ; N=426$

Signifikanz: ${ }^{*} p<0.05 ;{ }^{* *} p<0.01 ; * * * p<0.001$

In der Reihenfolge der Signifikanz lassen sich folgende Interpretationen signifikanter Zusammenhänge für die professionellen Bürokratien ableiten:

1 Bei einem hohen Maß an interner Personalveränderung (Mobilitätsdynamik) in professionellen Bürokratien erfolgt die Stellenbesetzung überwiegend intem.

Auch bei dieser Analyse wurden die Ergebnisse der Regressionsanalyse ergänzend mit Hilfe der Diskriminantenanalyse überprüft (siehe Anlage, Tab. 51). Das Resultat der Diskriminantenanalyse zeigt eine analysenspezifische Bestätigung.

Die beiden folgenden Resultate repräsentieren schwache Befunde, da die Pearsonkorrelationen das Ergebnis nicht signifikant unterstützen. 
2 Bei einem hohen $\mathrm{Maß}$ an Fluktuation erfolgt die Stellenbesetzung in den professionellen Bürokratien in der Regel intern.

Diese Beziehung steht ebenfalls im Gegensatz zu dem Resultat der Pearsonkorrelation. Dies läßt sich aus der Tatsache erklären, daß in der Regressionsanalyse von der Variable 'ONTVAC' ein starker Einfluß auf die anderen beiden Variablen ausgeht. Dies erklärt das negative Vorzeichen in Bezug auf die Variablen 'FKT' und 'VERL'.

3 Soweit es sich bei den offenen Stellen in professionellen Bürokratien um ein hohes Funktionsniveau handelt, werden diese organisationsintern besetzt.

Dieses Ergebnis bezieht sich auf Führungspositionen. Diese Positionen werden 'aus eigenen Reihen' besetzt. Es werden in der Regel Personen rekrutiert, die 'Führungserfahrung' mitbringen (Baron et al., 1986; Bills, 1987) und mit der 'eigenen' Organisation vertraut sind.

Nun gilt die Aufmerksamkeit der Regressionsanalyse bei den Maschinenbürokratien, wobei folgende Befunde abgeleitet werden können:

Tab. 52: Besetzung offener Stellen: Maschinenbürokratien Pearsonkorrelation; Regressionsanalyse (vervul als abhängige Variable)

\begin{tabular}{l|c|c|} 
Variablen & $\begin{array}{l}\text { Pearson- } \\
\text { korrelation }\end{array}$ & $\begin{array}{l}\text { Regressions- } \\
\text { analyse Beta }\end{array}$ \\
\hline 1 fkt & - & - \\
2 verl & 0.13 & $0.05 * *$ \\
3 ontvac & -0.93 & $-0.93 * *$
\end{tabular}

$\mathrm{r}^{2}=0.87 ;$ adj. $\mathrm{r}^{2}=0.85 ; \mathrm{N}=535$

Signifikanz: ${ }^{*} p<0.05 ;{ }^{* *} p<0.01 ;{ }^{* * *} p<0.001$

Die Interpretation der signifikanten Zusammenhänge bei den Maschinenbürokratien lautet wie folgt:

1 Bei einem hohen Maß intemer Personalveränderungen in Maschinenbürokratien vollzieht sich die Stellenbesetzung überwiegend intem.

Die deutliche Dominanz einer internen Mobilitätsdynamik in Maschinenbürokratien, wie es typisch ist für interne Arbeitsmärkte, führt dazu, daß offene Stellen aus dem eigenen Personalbestand besetzt werden.

2 Bei hoher Fluktuation in Maschinenbürokratien dominiert die externe Stellenbesetzung vor der internen Stellenbesetzung.

Dieses Ergebnis zeigt potentiell auf, daß bei einer hohen Fluktuation ein Zwang bestehen kann, Stellenbesetzungen in erster Linie durch externe Rekrutierungen vollziehen. 
Kein Zusammenhang besteht zwischen der abhängigen Variable und der unabhängigen Variable 'Funktion'.

Die als Ergänzung durchgeführte Diskriminantenanalyse (Anlage, Tab. 53) bestätigt die Ergebnisse analysenspezifisch.

Im folgenden schließt sich eine zusammenfassende Ergebnisanalyse an, die auf den Regressionsanalysen beruht.

Tab. 54: Zusammenfassende Ergebnisübersicht der offenen Stellenanalyse auf der Basis der Regressionsanalyse

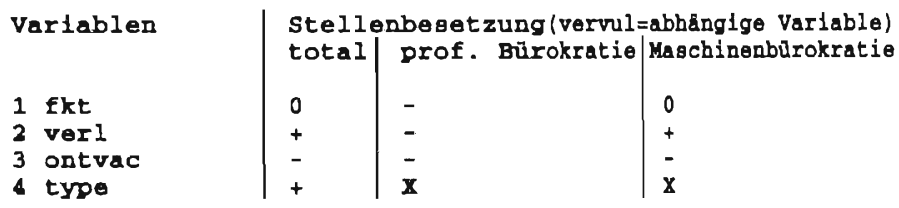

Erläuterung: +=positiver Zusamenhang; -=negativer Zusammenhang; $0=$ kein Zusammenhang; $\mathrm{X}=$ nicht untersucht

Die Frage, welche Rekrutierungspräferenzen bei der grundsätzlichen Alternative intemer/ externer Besetzung im Mittelpunkt stehen, kann durch die folgenden Ergebnisse beantwortet werden. Alle Analysen zeigen eine Gemeinsamkeit: in einer Situation großer intemer personeller Veränderung erfolgt die Stellenbesetzung aus intemer Besetzung. Unterschiede zeichnen sich bei den drei Analysen in Bezug auf 'Fluktuation' ab. Bei einem hohen Maß an Fluktuation erfolgt die Stellenbesetzung für die Totalanalyse und die Maschinenbürokratien extern, bei den professionellen Bürokratien intem.

Die Ergebnisse hinsichtlich der Variablen 'Funktion' und 'Type' zeigen kein konsistentes Interpretationsmuster. Aufgrund dieser Prüfung kann Hypothese 6 nicht bestätigt werden. Anhaltspunkte für eine Bestätigung von Hypothese 6 lassen sich aus dem deskriptiven Teil der Untersuchung ableiten (Tabelle 37).

\subsection{Die interne Besetzung freiwerdender Stellen}

In diesem Abschnitt geht es um die Betrachtung der intemen Besetzung offener Stellen einschlieBlich der organisatorischen Besonderheiten.

\subsubsection{Interne Stellenbesetzungen}

Dieser Abschnitt hat die Analyse der intemen Stellenbesetzung zum Gegenstand (Hypothesen 7 u. 8). Neben der Totalanalyse steht die Differenzienung nach Organisationskonfigurationen zentral. Die Übersetzung des Zahlenmaterials in Worte erfolgt im Anschluß an die Zusammenfassung der Ergebnisse in der jeweiligen Übersicht. 
Die folgenden Zahlen spiegeln die Ergebnisse für die drei Analyseeinheiten wider. Aufgrund einer Multikollinearität der Variablen KETEN, ZELBER und ANBER (siehe Anlage, Tab. 55-57) wurde von der üblichen Präsentation der Resultate abgewichen. Parallel zu der üblichen Regressionsanalyse (I) wurden Regressionsanalysen (II bis IV) durchgeführt unter Weglassen von Variablen, die von einer hohen Interkorrelation betroffen sind. Ziel dabei ist es, den Einfluß einer anderen Variablenkonstellation zu prüfen. Zusätzlich wurden die 'adjusted R Square'-Werte einbezogen, die Ausdruck der Beständigkeit sind bei unterschiedlichem Variableneinfluß.

Die folgenden Zusammenhänge intemer Stellenbesetzung lassen sich als Ergebnis der Untersuchung festhalten.

Tab. 58: Interne Besetzung offener Stellen: total; Pearsonkorrelation; Regressionsanalyse (dip als abhängige Variable)

\begin{tabular}{|c|c|c|c|c|c|}
\hline Variablen & $\begin{array}{l}\text { Pearson- } \\
\text { korre- } \\
\text { lation }\end{array}$ & $\begin{array}{l}\text { Regressio } \\
\text { I } \\
\text { alle }\end{array}$ & $\begin{array}{l}\text { onganalyse } \\
\begin{array}{|l}\text { II } \\
\text { RETEN } \\
\text { ANBER }\end{array}\end{array}$ & $\begin{array}{l}\text { a Beta } \\
\text { III } \\
\text { ZELBBR } \\
\text { ANBER }\end{array}$ & \begin{tabular}{|l} 
IV \\
nuI \\
KETEN
\end{tabular} \\
\hline $\begin{array}{ll}1 & \text { FKT } \\
2 & \text { VBRL } \\
3 & \text { ONTVAC } \\
4 & \text { SAL } \\
5 & \text { RBTBN } \\
6 & \text { ZBLBBR } \\
7 & \text { ANBBR } \\
8 & \text { TYPB }\end{array}$ & $\begin{array}{c}0.14 \\
0.49 \\
-0.15 \\
-0.09 \\
0.24 \\
0.38 \\
- \\
-0.53\end{array}$ & $\mid \begin{array}{c}- \\
0.26 \cdots * \\
- \\
- \\
-0.16 \\
0.15 \\
- \\
-0.33 * \cdots *\end{array}$ & $\left|\begin{array}{c}- \\
0.26 * * \hbar \\
- \\
- \\
- \\
\operatorname{xxxxx} \\
- \\
-0.38 \cdots * *\end{array}\right|$ & $\mid \begin{array}{c}- \\
0.22 \star * t \\
- \\
- \\
\operatorname{xxxxx} \\
- \\
- \\
-0.35 \star *\end{array}$ & $\mid \begin{array}{c}- \\
0.28 \cdots \\
- \\
- \\
- \\
\operatorname{xxxxx} \\
\operatorname{xxx\times x} \\
-0.34\end{array}$ \\
\hline $\begin{array}{l}r^{2}= \\
\text { adj. } r^{2}= \\
N=\end{array}$ & & $\begin{array}{l}0.34 \\
0.33 \\
535\end{array}$ & $\begin{array}{l}0.33 \\
0.32 \\
535\end{array}$ & $\begin{array}{l}0.33 \\
0.32 \\
535\end{array}$ & $\begin{array}{l}0.33 \\
0.32 \\
535\end{array}$ \\
\hline
\end{tabular}

$\mathrm{XXXXX}=$ Variable in Analyse ausgeschlossen Signifikanz: ${ }^{*} p<0.05 ;{ }^{* *} p<0.01 ;{ }^{* * *} p<0.001$ 
Bei den professionellen Bürokratien ergibt sich folgendes Bild:

Tab. 59: Besetzung offener Stellen: prof. Burokratien; Pearsonkorrelation; Regressionsanalyse (dip als abhängige Variable)

\begin{tabular}{|c|c|c|c|c|c|}
\hline \multirow[t]{2}{*}{ Variablen } & \multirow{2}{*}{$\begin{array}{l}\text { Peareon- } \\
\text { korre- } \\
\text { lation }\end{array}$} & \multicolumn{4}{|c|}{ Regressionsanalyse Beta } \\
\hline & & $\begin{array}{l}\text { I } \\
\text { alle }\end{array}$ & \begin{tabular}{|l} 
II \\
RBTEN \\
ANBER
\end{tabular} & \begin{tabular}{|l} 
III \\
ZBLBER \\
ANBBR
\end{tabular} & \begin{tabular}{|l} 
IV \\
nur \\
XETBN
\end{tabular} \\
\hline 1 PKT & - & - & - & - & - \\
\hline 2 VBRL & - & - & - & - & - \\
\hline 3 ONTVAC & - & - & - & - & - \\
\hline 4 SAL & - & - & - & - & - \\
\hline 5 RBTEN & 0.26 & - & $0.26 *$ & $x \times x \times x$ & $-0.26 *$ \\
\hline 6 ZELBER & 0.32 & $0.29 \star$ & $\mathbf{x x x} \times \mathbf{x}$ & $0.30 * *$ & $\operatorname{xxxxx}$ \\
\hline 7 ANBBR & 0.28 & $0.28 * \hbar *$ & $0.28 \cdots *$ & $0.28 * * *$ & $x x x x x$ \\
\hline$x^{2}=$ & & 0.19 & 0.15 & 0.17 & 0.08 \\
\hline $\begin{array}{l}a d j \cdot I^{2}= \\
N=\end{array}$ & & $\begin{array}{l}0.15 \\
149\end{array}$ & $\begin{array}{l}0.12 \\
149\end{array}$ & $\begin{array}{l}0.14 \\
149\end{array}$ & $\begin{array}{l}0.05 \\
109\end{array}$ \\
\hline
\end{tabular}

XXXXX $=$ Variable in Analyse ausgeschlossen

Signifikanz: * $\mathrm{p}<0.05 ;{ }^{* *} \mathrm{p}<0.01 ;{ }^{* * *} \mathrm{p}<0.001$

Bei den Maschinenbïrokratien zeichnet sich folgendes Bild ab:

Tab. 60: Besetzung offener Stellen: Maschinenbürokratien; Pearsonkorrelation; Regressionsanalyse (dip als abhängige Variable)

\begin{tabular}{|c|c|c|c|c|c|}
\hline Varlablen & Peargon- & Regresilo & oneanalyge & e Beta & \\
\hline & $\begin{array}{l}\text { korre- } \\
\text { lation }\end{array}$ & $\begin{array}{l}I \\
\text { allo }\end{array}$ & \begin{tabular}{|l} 
II \\
RBTBN \\
ANBER
\end{tabular} & \begin{tabular}{|l} 
III \\
ZBLBER \\
ANBER
\end{tabular} & \begin{tabular}{|l} 
IV \\
nur \\
RBTEN
\end{tabular} \\
\hline 1 FKT & - & - & - & - & - \\
\hline 2 VERL & 0.26 & $0.36 * *$ & $0.33 * * *$ & $0.32 * *$ & $0.35 *$ \\
\hline 3 ONTYVAC & -0.13 & $-0.15 *$ & $-0.15 *$ & $-0.15 \cdots$ & $-0.15 *$ \\
\hline A SAL & -0.09 & $-0.14 *$ & $-0.14 *$ & -0.13 & -0.14 \\
\hline 5 KRTEN & 0.10 & $-0.44 *$ & -0.19 & $x \times x x x$ & -0.13 \\
\hline 6 ZELBER & 0.13 & 0.29 & $\mathbf{X x x \times x}$ & - & $x x x x x$ \\
\hline 7 ANBER & 0.16 & - & - & - & $8 x \times x \times$ \\
\hline$r^{2}=$ & & 0.13 & 0.11 & 0.10 & 0.11 \\
\hline $\begin{array}{l}\text { adf. } r^{2}= \\
N=\end{array}$ & & $\begin{array}{l}0.11 \\
386\end{array}$ & $\begin{array}{l}0.10 \\
386\end{array}$ & $\begin{array}{l}0.09 \\
386\end{array}$ & $\begin{array}{l}0.10 \\
386\end{array}$ \\
\hline
\end{tabular}

$\mathrm{XXXXX}=$ Variable in Analyse ausgeschlossen

Signifikanz: * $p<0.05 ;{ }^{* *} p<0.01 ; * * * p<0.001$

Die Pearsonkorrelation der Variable ZELBER mit der Variable DIP ist schwach ausgeprägt. Die Ergebnisse für die Maschinenbürokratien zeigen für die Variablen VERL, ONTVAC und SAL eine nur in Nuancen abweichende Signifikanz bei allen Regressionsanalysen. Die Variable KETEN ist bei Analyse I deutlich signifikant. Bei Wegfall der Variable ZELBER in Analyse II und IV ist KETEN schwächer signifikant. Unter dem Vorbehalt dieser analysetechnischen Begrenzung werden die Ergebnisse von Analyse I 
Gegenstand der ausführlichen Ergebnisbetrachtung. Zur Erhöhung der Transparenz werden die bisher vorgestellten Einzelergebnisse in folgender Tabelle integriert.

Tab. 61: Zusammenfassende Ergebnisübersicht der offenen Stellenanalyse bei interner Stellenbesetzung

\begin{tabular}{|c|c|c|c|}
\hline \multirow[t]{2}{*}{ Variablen } & \multicolumn{3}{|c|}{ 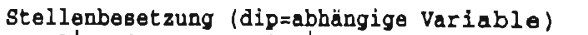 } \\
\hline & total & prof. Būrokratie & Maschinenbúrokratie \\
\hline 1 PRT & 0 & 0 & 0 \\
\hline 2 VERU & + & 0 & + \\
\hline 3 ONTVAC & 0 & 0 & - \\
\hline$\triangle \mathrm{SAL}$ & 0 & 0 & - \\
\hline 5 RBTEN & - & 0 & - \\
\hline 6 ZELBBR & + & + & + \\
\hline 7 ANBER & 0 & + & 0 \\
\hline 8 TYPB & - & $\mathbf{X}$ & $\mathbf{X}$ \\
\hline
\end{tabular}

Erläuterung: +=positiver Zusamenhang; -=negativer Zusammenhang;

$0=$ kein Zusammenhang; $X=$ nicht untersucht

Bei der Interpretation der Ergebnisse wird folgende Struktur gehandhabt. Zunächst werden Gemeinsamkeiten herausgearbeitet, die für alle Analysen gelten, danach wird spezifisch eingegangen auf Unterschiede, die Maschinenbürokratien und professionelle Bürokratien betreffen. Faßt man die quantitativen Ergebnisse in Worte, so ergeben sich die folgenden Zusammenhänge:

1 Für alle drei Analyseeinheiten läßt sich sagen, daß bei vielen potentiellen Kandidaten, die aus derselben Berufsgruppe entstammen, diese vorwiegend organisationsweit gesucht werden.

Für die Maschinenbürokratien gelten die folgenden Ergebnisse:

2 Bei hoher Fluktuation der Arbeitnehmer besteht die Tendenz, Personen organisationsweit für die Besetzung der offenen Stelle zu suchen.

Dieses Ergebnis zeigt, daß die Wirkung von intemen organisatorischen Grenzen in bestimmtem Maße für die Besetzung der offenen Stelle außer Kraft gesetzt wird. Das potentielle Suchfeld wird dadurch vergrößert einschließlich der Chance, eine geeignete Person zu finden. Hohe Fluktuation veranlaßt das Personalmanagement, von gewöhnlichen internen Besetzungsmustern abzuweichen.

3 Mit Einschränkung auf die Maschinenbürokratien ist abzuleiten, daß bei umfangreichen internen Personalveränderungen die Stellenbesetzung Resultat einer Suche ist, die sich auf die unmittelbare inteme Organisationsumgebung bezieht.

Dieses Ergebnis kennzeichnet die inteme Flexibilität, mit der in Maschinenbürokratien die Allokation erfolgt. Eine hohe interne Personalbewegung kann dazu beitragen, daß bei der offenen Stelle mehrere Personen aus der direkten Umgebung der zu besetzenden Stelle als 
Kandidaten in Frage kommen. Hier spielt die Besetzungsdichte der Funktionskette eine Rolle.

4 Für die Maschinenbürokratien trifft zu, daß eine geringe Dotierung der Stelle eine breitere innerorganisatorische Suche in der Regel nach sich zieht.

Ein geringes Gehalt charakterisiert eine Position, bei der die Suche nach einem potentiellen Kandidaten sich innerhalb der organisatorischen Grenzen vollzieht. Weil diese Funktionen noch wenig betriebsspezifische Erfahnungen voraussetzen, erfolgt die Rekrutierung aus einem weiten organisatorischen 'Umfeld'. Bei Maschinenbürokratien sind diese Funktionen im Vorfeld von 'Spezialisienungslaufbahnen' angesiedelt, die in dieser Form bei professionellen Bürokratien nicht anzutreffen sind.

5 Der folgende signifikante Zusammenhang bezieht sich ebenfalls auf die Analyseinheit der Maschinenbürokratien. Befinden sich viele potentielle Kandidaten in einer Funktionskette, so erfolgt die Stellenbesetzung meistens aus derselben Abteilung und Funktionskette, d. h. aus der nächsten Umgebung.

Dieses Ergebnis unterstreicht die Rolle der Funktionskettenallokation (Baron et. al., 1986; Althauser/Kalleberg, 1990). Bei der Anordnung von Funktionen in Form der Funktionskette besteht in erster Linie die Präferenz, um die offene Stelle mit Kandidaten aus der Funktionskette zu besetzen. Mit diesem Ergebnis wird Hypothese 7 bestätigt.

6 Speziell bei Maschinenbürokratien erfolgt im Gegensatz zu professionellen Bürokratien die Stellenbesetzung vorwiegend aus der 'nächsten Umgebung' d. h., derselben Abteilung und Funktionskette.

Die Ergebnisse der organisationsintemen Besetzung offener Stellen zeigen zugleich die Prioritäten der Allokation auf. Hier wird ein organisationsspezifisches Rekrutierungsfeld sichtbar, bei dem Nähe oder Weite des Kandidaten von der $z u$ besetzenden Position die Reallokationsentscheidung mitbeeinfluBt.

7 Spezifisch für die professionellen Bürokratien läßt sich ableiten, daß wenn für die interne Stellenbesetzung viele potentielle Kandidaten aus anderen Berufsgruppen zur Verfügung stehen, diese organisationsweit gesucht werden.

Die Einbeziehung von Kandidaten aus 'anderen Berufsgruppen' stellt nicht die für professionelle Organisationen erforderliche Standardqualifikation in den Mittelpunkt. Der Suchrahmen bei der internen Stellenbesetzung ist breiter angelegt. Bei diesem Resultat komm! zum Ausdruck, daß wenn der Suchrahmen wenig kandidatenspezifisch definiert ist, die interne Suche breit, d. h. organisationsweit erfolgt. Hier wird Hypothese 8 bestätigt. 


\subsection{In- bzw. externe Stellenbesetzung nach Parts}

Zum Schluß dieses Kapitel soll die Aufmerksamkeit auf die Einteilung der Organisation nach Parts gelenkt werden. Dabei steht die Frage im Vordergrund, welches sind die in den untersuchten Organisationen meist frequentierten Parts sowohl unter der Perspektive der in- als auch extemen Stellenbesetzung?

Tab. 62: Offene Stellenbesetzung auf dem organisationsinternen

Arbeitsmarkt im Dreijahreszeitraum 1986-1988 nach Parts

\begin{tabular}{|c|c|c|c|c|c|c|c|c|c|c|c|c|}
\hline \multirow{2}{*}{$\begin{array}{l}0 \\
r \\
g . \\
\text { Part }\end{array}$} & \multicolumn{10}{|c|}{$\begin{array}{l}\text { ste } 1 \text { le } a b e s e t z u n g \\
\text { extern }\end{array}$} & \multicolumn{2}{|c|}{ total } \\
\hline & 1 & 2 & 3 & 4 & 5 & 1 & 2 & 3 & 4 & 5 & & \\
\hline $\mathbf{K}$ & - & 5 & 150 & - & 89 & - & 3 & 110 & - & 24 & 381 & $(40)$ \\
\hline $\mathrm{W}$ & - & - & 28 & - & 6 & 1 & 4 & 6 & - & - & 45 & (5) \\
\hline $\mathrm{S}$ & - & 2 & 70 & 1 & 14 & - & 10 & 154 & - & 6 & 257 & $(27)$ \\
\hline$P$ & 1 & 2 & 15 & 6 & 38 & - & 8 & 111 & 10 & 88 & 279 & (29) \\
\hline $\begin{array}{l}t \\
q\end{array}$ & $\begin{array}{l}1 \\
(0)\end{array}$ & $\begin{array}{c}9 \\
(1)\end{array}$ & $\begin{array}{l}263 \\
(27)\end{array}$ & $\begin{array}{c}7 \\
(1)\end{array}$ & $\begin{array}{l}147 \\
(15)\end{array}$ & $\begin{array}{l}1 \\
(0)\end{array}$ & $\begin{array}{l}25 \\
(3)\end{array}$ & $\begin{array}{l}381 \\
(40)\end{array}$ & $\begin{array}{l}10 \\
\text { (1) }\end{array}$ & $\begin{array}{l}118 \\
(12)\end{array}$ & 962 & $\begin{array}{l}(100) \\
(100)\end{array}$ \\
\hline
\end{tabular}

Erläuterung: Die Prozentwert befinden sich in Klammern. $\mathrm{K}=$ Krankenhaus; S=Suzialversicherung; $\mathrm{W}=$ Wirtschaftsprüfungsunternehmen; $\mathrm{P}=$ Produktionsuntemehmen; $\mathrm{t}=$ total; Org.=Organisation; $1=$ Strategic Apex; $2=$ Middle Line; $3=$ Operating Core; $4=$ Technostructure; 5=Support Staff

Aus der Tabelle geht hervor, daß die meisten Stellenbesetzungen sowohl in- als auch extern im Part 'operating core' anfallen. Dies hängt unter anderem davon $a b$, daß in der Regel sich im 'operating core' ein großer Teil der Beschäftigten konzentriert. Die Vakanzenanalyse zeigt deutlich, daß die 'ports of entry' nicht beschränkt sind auf Part 'operating core'. Die Ergebnisse wie sie hier tabellarisch vorliegen, weisen darauf hin, daß die 'ports of entry' auch bei den Parts 'technostructure' und 'support staff' anzutreffen sind, unabhängig von der organisationsspezifischen Verteilung.

Analog wird in Bezug auf die Parts deutlich, daß der Organisationszugang prinzipiell zu allen Parts möglich ist. Allein die Zahlen verweisen auf die Häufigkeit, mit der der Zugang erfolgt. In der Reihenfolge der Häufigkeit der Stellenbesetzung folgen 'Support Staff', 'Middle Line', 'Technostructure' und schließlich 'Strategic Apex'. Bei dieser Betrachtung handelı es sich um eine statische Betrachtung, die nicht der Mobilitätsdynamik Rechnung trägt.

Unter dem Aspekt der Segmentation betriebsintemer Arbeitsmärkte und den damit verbundenen beschäftigungspolitischen und organisatorischen Grenzen geben die Ergebnisse Anlaß zu einer differenzierteren Beurteilung und Neubewertung des alten Phänomens der 
Aufteilung des betrieblichen Arbeitsmarktes in zwei oder drei Segmenten (Doeringer/Piore, 1971; Schwan/Soeters, 1991 b).

\subsection{Schlußfolgerungen}

Gegenstand dieses Kapitels war die Besetzung offener Stellen innerhalb der Organisationen. Zwei Analysen standen hier im Mittelpunkt. Zunächst ging es um die Erschließung der Stellenbesetzungen aus in- und extemen Rekrutierungsquellen. Danach konzentrierte sich das Erkenntnisinteresse auf die Stellenbesetzung aus ausschlieBlich internen Rekrutierungsquellen. Vor diesem Hintergrund und in dieser Reihenfolge gilt es, die Schlußfolgerungen zu ziehen.

Das Verhältnis in- und extemer Arbeitsmarkt wird durch folgende Einsichten geprägt. Die Besetzung offener Stellen ist bei beiden Organisationskonfigurationen gekennzeichnet durch ein Interaktionsmuster von in- und externen Rekrutierungen. Schaut man spezifisch nach der jeweiligen Organisationskonfiguration, dann dominiert bei Maschinenbürokratien die Zahl der internen Rekrutierungen, bei professionellen Bürokratien die Zahl der extemen Rekrutierungen. Aufgrund dieser Gegebenheiten wird deutlich, daß in Maschinenbürokratien mehr interne Mobilitätsdynamik anzutreffen ist als in professionellen Bürokratien, deren Stellenbesetzungsmuster auf einen stärkeren Austausch mit dem externen Arbeitsmarkt angelegt sind. Damit wird Hypothese 6 bestätigt.

Schaut man spezifisch nach den Allokationsmustem bei der Besetzung offener Stellen und den darin ausgedrückten Besetzungspräferenzen, dann sind die folgenden SchluBfolgenungen gerechtfertigt. Gemeinsam ist allen Untersuchungseinheiten, daß bei einer hohen internen Mobilitätsdynamik eine Präferenz in Richtung interner Stellenbesetzung besteht. Für die jeweilige Organisationskonfiguration ergeben sich Anhaltspunkte für folgende weniger starke Zusammenhänge. In professionellen Bürokratien werden in der Regel Stellen auf hohem Funktionsniveau intem besetzt. Bei hoher Fluktuation geht die Besetzungspräferenz vielfach in Maschinenbürokratien in Richtung externer Arbeitsmarkt, während bei den professionellen Bürokratien gewöhnlich eine interne Besetzung vor kommt.

Im letzten Ergebnisteil des Kapitels konzentrierte sich das Erkenntnisinteresse auf die ausschließlich interne Besetzung offener Stellen. Dabei ging es nicht allein darum, welche offene Stelle mit welcher Priorität besetzt wird, sondern die innerorganisatorischen (Mobilitïts-) Grenzen zu bestimmen, die die interne Allokation neben anderen Faktoren beeinflußen.

Danach verfügen Maschinenbürokratien über eine Tendenz, aus der 'unmittelbaren Umgebung' zu rekrutieren, d. h. aus derselben Abteilung und derselben Funktionskette. Hier prägen zwei für die Allokation wichtige innerorganisatorische Grenzen die Stellenbesetzung, nämlich die der Abteilung und die der Funktionskette. Hypothese 7 zielte auf diesen Sachverhalt der Mobilitätskettenallokation ab. Hypothese 7 kann als bestätigt angesehen werden. Beide Organisationsgrenzen haben einen starken Einfluß auf die Allokationsentscheidung. Sie erleichtern Personen innerhalb dieser Grenzen unter bestimmten vielfach stark institutionalisierten Bedingungen den Zugang zu einer höheren Funktion innerhalb 
der Jobleiter/Abteilung. Führt die Kombination dieser organisatorischen Grenzen zu keiner Allokationsentscheidung, dann erweitert man die Suchaktivitäten bezogen auf die gesamte interne Organisation.

Bei den professionellen Bürokratien läßt sich ein anderes Stellenbesetzungsmuster feststellen. Hier besteht kein so enges Stellenbesetzungsmuster aus der Kombination selbe Abteilung/selbe Funktionskette. Bei der Stellenbesetzung in professionellen Bürokratien dominiert ein Rekrutierungsmuster aus anderen Abteilungen und derselben Funktionskette. Dies war Gegenstand der Überprüfung von Hypothese 8, die als bestätigt betrachtet werden kann. Die inteme Personalsuche ist breiter (bezogen auf die Organisation) angelegt, d. h. man rekrutiert aus mittelbarer intemer Organisationsumgebung. Dies ist zu erklären mit der standardisierten Qualifikation der Mitarbeiter, die damit auch innerorganisatorisch besser übertragbar ist. Entscheidend für die Stellenbesetzung in professionellen Bürokratien ist eine enge Koppelung von Fachqualikation und Funktionskette. Fachfremde Qualifikation verdient in professionellen Bürokratien nur eine geringe Priorität bei der Stellenbesetzung.

Vergleicht man nun die Ergebnisse dieser Studie mit der DiPretes, dann lassen sich von den Resuitaten her gesehen in der Tendenz deutliche Übereinstimmungen feststellen. Dies betrifft insbesondere die Rekrutierungsmuster erster Suchpriorität in Maschinenbürokratien, d. h. Rekrutierung aus derselben Funktionskette/Abteilung. Während in DiPretes Studie unterschiedliche Funktionsketten für administrative und professionelle Funktionen nebeneinander in einer Organisation bestehen, sind diese in dieser Studie gekoppelt an die jeweilige Organisationskonfiguration. Dadurch sind eindeutigere Aussagen in Bezug auf den internen Arbeitsmarkt ableitbar.

Konkret bedeutet dies, daß Maschinenbürokratien von den Stellenbesetzungsmustern und den organisatorischen Grenzen ein deutliches Beschäftigungsprofil bilden, das typisch ist für inteme Arbeitsmärkte. Darüberhinaus kann hier empirisch der Nachweis erbracht werden, daß die Organisationsstruktur bzw. die Funktionsstruktur sowie interne und die exteme Organisationsgrenze( $n$ ) in den Organisationskonfigurationen einen deutlichen Einfluß auf die organisationsinteme Arbeitnehmermobilität haben.

Zu den allgemeinen Schlußfolgerungen dieses Kapitels läßt sich rechnen, daß das von Williamson benutzte Bild der 'protected govemance structure' auf unterschiedliche Weise hier zutrifft. Ziel dabei ist es, die aufgebauten Qualifikationspotentiale der Mitarbeiter in Form des intemen Arbeitsmarktes zu erhalten. Es wird deutlich, daß die 'human asset specifity' für die jeweilige Organisationskonfiguration typisch ist und damit die Allokation direkt beeinflußt.

Brittain/Wholey (1990) unterstreichen die Nüzlichkeit von 'boundary-spanning options'. Durch die Schaffung organisatorischer 'Untereinheiten' schützt die Organisation know how und federt dadurch die eigenen Aktivitäten gegenüber Einflüssen von außen ab. Diese Sichtweise läßt sich auf den Bereich der internen Organisation bzw. interner Arbeitsmärkte übertragen. 
Die Ergebnisse bestätigen die Charakteristiken des intemen Arbeitsmarktes bei der Besetzung offener Stellen in Bezug auf die Maschinenbürokratien. Bei den professionellen Bürokratien sind ebenfalls Züge bzw. Kennzeichen intemer Arbeitsmärkte zu finden.

Die Ergebnisse der offenen Stellenanalyse bieten eine Ausgangssituation für weitere spezifizierte Analysen zur Erschließung mikroorganisationaler (mikrostruktureller) Allokationskriterien. Die Besetzung offener Stellen basiert in dieser Studie auf der Annahme des Einflusses von institutionalisierten Organisationsstrukturen (Brandes/Weise, 1987).

Im folgenden Schlußkapitel werden die Ergebnisse der Untersuchung zusammengefaßt, eine Rückkoppelung zu den theoretischen Ausgangspositionen gelegt und Schlußfolgerungen abgeleitet. 


\section{Zusammenfassung und Schlußfolgerungen aus der Untersuchung}

In diesem Kapitel werden die wichtigsten konzeptionellen Schritte der Studie sowie die Untersuchungsergebnisse zusammengefaßt. Außerdem erfolgt hier eine Rückkoppelung zur zentralen Fragestellung. Daneben wird der Beitrag, den diese Studie in Bezug auf die Segmentationsproblematik betrieblicher Arbeitsmärkte leistet, herausgearbeitet. Ferner stellt sich die Frage, ob die Ergebnisse auf eine organisationsspezifische Beschäftigungsstrategie hindeuten.

Außerdem wird geprüft, worin der Beitrag der empirischen Untersuchung in Bezug auf die theoretischen Ausgangsvoraussetzungen besteht. Kurz eingegangen wird auf Forschungsperspektiven, die im Rahmen dieser Studie nicht behandelt werden konnten bzw. Aspekte eröffnen für weitere Forschungsaktivitäten. Dieses Kapitel endet mit einer Zusammenfassung in niederländischer und englischer Sprache.

\subsection{Zusammenfassung der wichtigsten Untersuchungsergebnisse}

\subsubsection{Das Konzept der Studie}

Die empirische Untersuchung konzentrierte sich auf den betrieblichen Arbeitsmarkt als Beschäftigungsform innerhalb von vier Untemehmensorganisationen bzw. Einrichtungen. Die Erkenntnisse sind auf der Basis des Vergleichs der einbezogenen Organisationen zustande gekommen. Ausgangspunkt ist die Theorie intemer Arbeitsmärkte von Doeringer/Piore. Sie diente der Erschließung organisationsstruktureller Eigenschaften des internen Arbeitsmarktes. Ziel war es, diese Arbeitsmarkttheorie zu kontrastieren mit den ebenfalls organisationsstrukturellen Eigenschaften betrieblicher Beschäftigung im Rahmen von Organisationstheorien. Die Auswahl der Organisationstheorien fiel auf die Transaktionskostentheorie und die Kontingenztheorie. Im Licht beider Theorien wurden die Kennzeichen intemer Arbeitsmärkte erarbeitet. Hinsichtlich der internen Arbeitmärkte konnte eine weitgehende konzeptionelle Übereinstimmung der untersuchungsrelevanten Merkmale beider Organisationstheorien erreicht werden. Bevor auf die Ergebnisse eingegangen wird, gilt es, die zentrale Fragestellung in Erinnerung zu rufen:

In welchem Maß unterscheiden sich Maschinenbürokratien und professionelle Bürokratien in der Prägnanz der Anwesenheit der angegebenen Beschäftigungskennzeichen von internen Arbeitsmärkten?

Als Ergebnis konnte aufgezeigt werden, daß interne Arbeitsmärkte im Rahmen dieser Untersuchung in Maschinenbürokratien und professionellen Bürokratien zu identifizieren 
sind. Jedoch hinsichtlich der Ausprägung von Beschäftigungskennzeichen begründen beide Organisationskonfigurationen unterschiedliche Muster des internen Arbeitsmarktes. Damit kann die zentrale Fragestellung hinsichtlich der Ausprägung des Unterschiedes der Beschäftigungskennzeichen beider Organisationskonfigurationen beantwortet werden. Die in Kapitel 1 aufgestellte hypothetische Annahme, daß Maschinenbürokratien mehr Affinität zur Hierarchie aufweisen, kann aufgrund der Art der Beschäftigungskennzeichen bestätig! werden. Die Auspägung der Beschäftigungskennzeichen bei den professionellen Bürokratien spricht für die Marktnähe innerhalb des Spektrums zwischen Hierarchie und Markl.

Das Erkenntnisinteresse richtete sich auf die Ebene der Organisationskonfiguration. Dahinter stand eine ganzheitliche Sichtweise der Organisation. Die Operationalisierung des Erkenntnisinteresses erfolgte in zwei Richtungen. Den vielseitigen Anfordenungen interner Arbeitsmärkte wurde in Form von zwei sich ergänzenden Analysen Rechnung getragen. Zum einen handelt es sich um eine Infrastrukturanalyse intemer Arbeitsmärkte, zum anderen um die Analyse der Besetzung offener Stellen. Die wichtigsten Resultate sollen hier zusammengefaßt werden.

\subsubsection{Die Infrastrukturanalyse interner Arbeitsmärkte}

Die Infrastrukturanalyse erstreckte sich auf drei Detailanalysen. Diese bestanden aus der Beförderungs-, der Gehaltszuwachs- und einer Beschäftigungsdaueranalyse.

Mit der Beförderungsanalyse konnte gezeigt werden, daß betriebsspezifische Weiterbildung die Chance auf Beförderung erhöht. Die Zahl der Beförderungen ist abhängig von der Länge der Dienstzeit. Im Vergleich zu den professionellen Bürokratien kommen in Maschinenbürokratien mehr Beförderungen vor. Damit konnte Hypothese 1, Kapitel 4 bestätigt werden. Die Resultate weisen auf die Kennzeichen des internen Arbeitsmarktes hin, die für die Maschinenbürokratien deutlicher ausgeprägt sind als für die professionellen Bürokratien.

Bei der Gehaltszuwachsanalyse (Hypothese 3) konnte bestätigt werden, daß die Arbeitnehmerbindung in Maschinenbürokratien mit einem höheren Gehaltszuwachs als in professionellen Bürokratien verbunden ist. Frauen partizipieren im Vergleich zu Männem in geringerem Maße von den Gehaltszuwächsen (Kalleberg u. a., 1981).

Die Beschäftigungsdaueranalyse zeigt für die Maschinenbürokratien, daß ein hohes Funktionsniveau mit einer langen intemen Beschäftigungszeit korrespondiert (Hypothese 4). Fluktuation tritt auf in der Anfangsphase der Organisationszugehörigkeit, d. h. es liegt eine kurze Beschäftigungsdauer zugrunde. Bestätigen ließ sich auch, daß Funktionswechsel in der Phase kurzer Beschäftigungsdauer öfter auftreten. Damit kann Hypothese 5 als bestätigt angesehen werden. Bei professionellen Bürokratien zeigt sich für Frauen eine kürzere Beschäftigungsdauer als bei Männem. Darüberhinaus ergeben sich hinsichtlich der Merkmale 'Funktionswechsel ' und 'Schulausbildungsniveau' Übereinstimmungen mit den Maschinenbürokratien.

Aufgrund der gefundenen Unterschiede zwischen den Organisationskonfigurationen kann man einen starken Einfluß auf die zukünftige Entwicklung der Beschäftigungszeit erwar- 
ten. So gehen Kerckhoffs/Wolfs (1991), basierend auf einer nationalen niederländischen Erhebung davon aus, daß im Gegensatz zu den niederländischen Durchschnittswerten (11 Prozent, 1985) der Beschäftigungszeil (der Gruppe der Beschäftigten von 20 und mehr Jahren) die zukünftige Beschäftigungsdauer von 20 und mehr Jahren von einer deutlich niedrigeren Zahl (rund 40 Prozent der Arbeitnehmer 1985) von Arbeitnehmem erreicht werden wird. Dies könnte tendenziell eine Abwertung von internen Arbeitsmärkten als die dafür typische Beschäftigungsform zur Folge haben. Gleichzeitig gilt es zu bedenken, daß nicht alle Formen langfristiger Beschäftigung sich auf internen Arbeitsmärkten vollziehen. Sofern im Dienstleistungssektor eine Zunahme an professionellen Bürokratien zu verzeichnen ist, bedeutet dies, daß die Vorhersagen in dieser Form als zu optimistisch zu betrachten sind. Der Wert dieser Studie liegt darin, daß die Verschiebungen in der $\mathrm{Zu}$ sammensetzung von Organisationsformen auf mögliche Effekte eingeschätzt werden können.

\subsubsection{Die Analyse der offenen Stellen}

Die offene Stellenanalyse hatte die Rekonstruktion von internen Stellenbesetzungen aus in- bzw. extemen Rekrutienungsquellen zum Gegenstand. Zwei Detailanalysen lagen dem zugrunde. Dabei handelt es sich um die Analyse wechselnder Inanspruchnahme der organisationsintermen bzw. der -extemen Rekrutierungsquellen bei der Besetzung offener Stellen. Bei Maschinenbürokratien dominiert die interne Rekrutierung gegenüber den professionellen Bürokratien, die Personal überwiegend vom externen Markt rekrutieren (Hypothese 6). Damit wird der Interaktion mit der 'Außenwelt' Rechnung getragen. Auf die ausschließlich inteme Besetzung offener Stellen war eine weitere Analyse gerichtet. Diese Analyse basiert auf einem Präferenzspektrum intemer Rekrutierung. Zugrunde liegt die Perspektive, daß die Präferenz der inlernen Rekrutierung zunächst aus der unmittelbaren intemen Organisationsumgebung in Maschinenburokratien erfolgt, in der der potentielle Kandidat bisher beschäftigt war. Bei professionellen Bürokratien dominiert die Rekrutienung aus dem mittelbaren organisationsinternen Umfeld. Mit diesem Ergebnis kann Hypothese 7 bestätigt werden.

Die Ergebnisse der ersten Analyse reflektieren das Verhälmis von intemer und externer Besetzung offener Stellen. In beiden Organisationskonfigurationen wird von beiden Formen der Besetzung offener Stellen Gebrauch gemacht. Bei den Maschinenbürokratien dominiert die interne Beselzung, bei den professionellen Bürokratien dominiert die externe Besetzung offener Stellen. Damit entsprechen die professionellen Bürokratien in geringerem Maße intemen Arbeitsmärkten als Maschinenbürokratien.

Daneben interessiente die Frage, welche Beschäftigungsmerkmale in welcher Form dic inbzw. externe Allokation beeinflussen. Im Hinblick auf eine hohe Fluktuation ergab sich das folgende Allokationsmuster. Im Zeichen der Interaktion der Besetzung offener Stellen zeigt sich für professionelle Bürokratien meist eine interne, für Maschinenbürokratien meist eine exteme Besetzung von offenen Stellen. Bei der ausschließlich internen Stellenbesetzung kommt für alle Organisationen eine Besetzungspräferenz zum Ausdruck, die eine organisationsweite Suche impliziert. 
Hinsichtlich einer hohen organisationsinternen Personalbewegung lassen sich folgende Allokationsmuster ableiten. Bei beiden Organisationskonfigurationen steht die inteme Besetzung von Stellen im Vordergrund, d. h., daß man nahezu keinen Gebrauch macht von extemen Rekrutierungen. Allgemein läßt sich daraus ableiten, daß unter dem Einfluß gefähurdeter Beschäftigungsstabilität (im Falle hoher Fluktuation bzw. hoher intemer Personalbewegung) eine interne Besetzungpräferenz Priorität erfährt. Die Analyse der Besetzung offener Stellen (bei ausschlieBlich intemer Rekrutierung) bietet für die Maschinenbürokratien eine Bestätigung der Präferenz für die Rekrutierung aus derselben Abteilung und derselben Funktionskette. Das bedeutet, daß in Maschinenbürokratien zunächst aus unmittelbarer organisatorischer Nähe der Vakanz der Kandidat für die Stellenbesetzung Präferenz erfährt.

Die Besetzung offener Stellen ist durch die Präferenz interner Rekrutierung in professionellen Bürokratien geprägt bei der grundsätzlichen Wahlmöglichkeit zwischen in- und externer Besetzung. Dieses Ergebnis ist zu sehen im Zeichen hoher intemer Personalveränderungen. Hier wird Hypothese 8 bestätigt.

Die folgenden Allokationsmuster beziehen sich allein auf die Analyse intemer Stellenbesetzungen.

Bei der Besetzung offener Stellen aus einer großen Zahl potentieller Bewerber in der Funktionskette ergeben sich die folgenden Resultate. Bei Maschinenbürokratien besteht bei einer großen Zahl von Personen aus derselben Funktionskette eine Präferenz zugunsten der Stellenbesetzung aus derselben Abteilung und derselben Funktionskette.

Die potentielle Besetzung offener Stellen mit Kandidaten, die einer anderen Berufsgruppe entstammen, deutet die folgenden Allokationsmuster an. Die Analyse bringt eine Präferenz in professionellen Bürokratien zugunsten von Personen zum Ausdruck, die aus einer anderen Berufsgruppe kommen.

Für die Maschinenbürokratien bestätigt sich, daB ein hohes Gehaltsniveau im Zusammenhang steht mit der Rekrutierungspräferenz aus unmittelbarer intemer Organisationsumgebung. Zusammenfassend werden an Hand der empirischen Ergebnisse deutliche Rekrutierungsmuster per Organisationskonfiguration sowohl nach außen als auch nach innen sichtbar. Bei professionellen Bürokratien findet mehr Interaktion mit dem externen Arbeitsmarkt statt (Buitendam, 1991), während für die Maschinenbürokratien eine deutliche Dominanz des internen Allokationsgeschehens typisch ist. Die Lage der 'ports of entry' in den untersuchten Organisationen ergänzt das Bild. Die 'ports of entry' in den professionellen Bürokratien verteilen sich über die bestehende Hierarchie, während bei den Maschinenbürokratien eine Konzentration im unteren Funktionshierarchiebereich anzutreffen ist. Mit diesem Ergebnis kann Hypothese 2, Kapitel 4 bestätigt werden.

Nachgewiesen wurde ferner der Zusammenhang von Organisationskonfiguration und Rekrutierungsstrategien (Windolf/Hohn, 1984) sowie die für den intemen Arbeitsmarkt typische und dominante Rekrutierungsstrategie. 
Die Untersuchung hat gezeigt, daß neben der Organisationsaußengrenze auch interne Organisationsgrenzen einen entscheidenden EinfluB auf die Allokation haben. Dieser EinfluB ist bei den Maschinenbürokratien stärker ausgeprägt als bei den professionellen Bürokratien. Der Effekt des Einflußes von intemen Organisationsgrenzen läßt sich als institutionalisiertes Allokationsmuster fixieren (Hoof, van; 1987). 'Störungsgrößen' wie Fluktuation oder interne Personalveränderungen, die die betriebsspezifische Beschäftigungsstabilität vorïbergehend in Frage stellen, beweisen, daß ein einmal institutionalisiertes Allokationsmuster nicht die Flexibilität modifizierter Anwendung ausschlieBt.

\subsubsection{Rückkoppelung zur zentralen Fragestellung}

Die Beurteilung soll sich wie folgt vollziehen. Zunächst werden im ersten Schritt entsprechend der Komplexität zur Beurteilung eine Reihe von Indikatoren herangezogen, die im Hinblick auf die Organisationskonfigurationen vergleichend beurteilt werden und dann in eine Gesamtbeurteilung münden.

Ziel dieses Abschnitts ist es, von einem 'schwachen theoretischen Verständnis interner Arbeitsmärkte' bzw. einem "uneasy amalgam of human capital theory" (Osterman, 1987, S. 46) Abstand zu nehmen und zu einer zusammenhängenden Erklärung zu kommen. Eine Besonderheit kann darin gesehen werden, daß interne Arbeitsmärkte bestehen aus einer "...collection of characteristics that occur together, none of which alone can be precisely predicted." (Mace, 1979, S. 51) Mit dieser Aussage wird eine erweiterte Betrachtungsweise interner Arbeitsmärkte nahegelegt. Diese erweiterte Betrachtung ist umfassender als der Ansatz, der in de Gijsel/Muysken/Wolfs (1991) zum Ausdruck kommt, die inteme Arbeitsmärkte durch zwei Kriterien charakterisieren: langfristige Arbeitsverträge und die Anwesenheit von Beförderungschancen.

Im folgenden Schritt gilt es, die jeweiligen Resultate mit Relevanz für den internen Arbeitsmarkt zu bewerten.

Doeringer/Piore (1971) geben keine 'harten' Kriterien an, mit deren Hilfe sich die Kriterien bzw. die Theorie des internen Arbeitsmarktes empirisch überprüfen läßt. Wir greifen daher zurück auf die Organisationskonfigurationen. Diese repräsentieren einen Zwischenschritt auf dem Weg der Identifikation intemer Arbeitsmärkte.

1 Interne Arbeitsmärkte zeigen ein bestimmtes $\mathrm{MaB}$ an Geschlossenheit was den Zugang und den Abstrom von Personal betrifft. Zieht man die Fluktuationsrate als Indikator für die 'Offenheit/Geschlossenheit' einer Organisation heran (Doeringer/Piore, 1971; Mace, 1979; Bergerijk/De Grip, 1986), dann wird aufgrund der ermittelten Fluktuationsquoten deutlich, daß sowohl Maschinenbürokratien als auch professionelle Bürokratien unter der von Alexander (1974) hantierten (ad hoc-) Norm von zehn Prozent liegen. Die Fluktuationsquoten drücken eine stabile Beschäftigungssituation aus. Allein mit diesem Indikator ist noch kein Maßstab für den intemen Arbeitsmarkt gesetzt.

2 Die Lage bzw. die Verteilung der 'ports of entry' kann als ein weiterer Indikator hinzugezogen werden. In Bezug auf die Organisationskonfiguration läßt sich ableiten, daß bei Maschinenbürokratien eine Konzentration der 'Eintrittspositionen' im unteren Hier- 
archiebereich anzutreffen ist im Gegensatz zu den professionellen Bürokratien, bei denen die 'Eintrittspositionen' ủber die Hierarchie der Organisation verteilt sind. Anhaltspunkte dafür bietet die Funktionsverteilung und das Rekrutierungsverhalten der jeweiligen Organisation. Bezogen auf die Charakteristiken intemer Arbeitsmärkte zeigt sich, daß die beiden Organisationskonfigurationen deutlich voneinander abweichen. Die Maschinenbürokratien kommen hinsichtlich dieses Indikators dem Idealbild intemer Arbeitsmärkte recht nahe, während die professionellen Bürokratien nur teilweise diesem Bild entsprechen. Bei letzteren zeigt sich mehr Markınähe und damit mehr Offenheit gegenüber den Maschinenbürokratien.

3 Charakteristisch für beide Organisationskonfigurationen sind die Aufstiegsleitem. Auf den ersten Blick ist darin eine Gemeinsamkeit beider Organisationskonfigurationen zu schen. Eine genauere Betrachtung weist jedoch erhebliche Unterschiede im Aufbau und Gestaltung der Aufstiegsleitern aus. So lassen sich diese bei professionellen Bürokratien im Gegensatz zu den Maschinenbürokratien im allgemeinen als kurz bezeichnen (Kapitel 6). In den professionellen Bürokratien sind diese gekoppelt an eine Fachqualifikation, deren Funktionsniveaus von nahezu allen Personen potentiell durchlaufen werden. Die Koppelung mit Fachqualifikation und dem daraus resultierenden Funktionsniveau besteht bei den Maschinenbürokratien in dieser Form allgemein nicht. Hier stehen organisaliunsspezifische Qualifikationen im Vordergrund, der Personaleinsatz ist dadurch flexibler. Die Aufstiegsleitem sind an ein von der Tendenz steigendes Alters-Einkommensprofil gebunden. Die Arbeitsplätze innerhalb der drei großen Organisationen sind Gegenstand einer Funktions- bzw. Arbeitsplatzbewertung gewesen.

$4 \mathrm{Zu}$ internen Arbeitsmärkten gehört die Koppelung von Funktion (vielfach in Form der Funktionskette) und interner Aus- und Weiterbildung. Bei Maschinenbürokratien ist die Koppelung deutlich ausgeprägt, bei professionellen Bürokratien etwas weniger akzentuiert. Die etwas schwächere Ausprägung bei der zuletzt genannten Organisationskonfiguration hängt zusammen mit den weniger betriebsspezifischen Aus- und Weiterbildungsinhalten.

5 Für beide Organisationskonfigurationen (empirisch) und für den intemen Arbeitsmarkt (theoretisch) gilt ein auf Dauer angelegtes Arbeitsverhältmis. Beförderungen berücksichtigen diese Zeitkomponente. Gemessen an diesem Ergebnis erfährt das Konzept der Arbeitnehmerbindung eine Bestätigung.

Zieht man eine Bilanz, so lassen sich deutliche Kontraste zwischen den beiden Organisationskonfigurationen feststellen, aber auch Übereinstimmungen. Eine Bewertung der Organisationskonfigurationen hinsichtlich des intemen Arbeitsmarktes kann nun erfolgen. Die in diesem Abschnitt angesprochenen Ergebnisse, aber auch jene Resultate der Detailanalysen zeigen, daß inteme Arbeitsmärkte nahezu übereinstimmen mit den Merkmalen der Maschinenbürokratien.

Protessionelle Bürokratien weisen in dieser Untersuchung einen 'Verwandischaftsgrad' zu intemen Arbeitsmärkten auf. Dies kommt zum Ausdruck in der Übereinstimmung von Merkmalen bei den beiden Organisationskonfigurationen. 
Professionelle Bürokratien in Form von intemen Arbeitsmärkten zeigen vielfach mehr 'Geschlossenheit' als durch die Marktnähe zu erwarten ist. Im Falle des Krankenhauses zeichnete sich eine solche Tendenz in Richtung interner Arbeitsmarkt deutlich ab. Unter dem Einfluß von anderen Beschäftigungskulturen, z. B. in den Vereinigten Staaten lassen sich beispielsweise professionelle Bürokratien antreffen, die vollkommen als interne Arbeitsmärkle organisiert sind (Dillard/Ferris, 1989; Osterman, 1988; Bills, 1987; Mace, 1979).

Die untersuchten Organisationen stimmten hinsichtlich der Beschäftigungskennzeichen weitgehend mit den theoretischen Organisationskonfigurationen überein. Allerdings zeigten sich in der Empirie auch Abweichungen. Beim Krankenhaus war eine deutliche Tendenz zur Maschinenbürokratie aufgnund einzelner Merkmalsausprägungen feststellbar. Im Produktionsunternehmen ergab sich bei den Funktionen eine Tendenz zur professionellen Bürokratie hinsichtlich eines größeren Bedarfs an technisch spezialisiertem Personal.

Bezogen auf die vorliegende Untersuchung, wir kommen damit auf die zentrale Fragestellung der Untersuchung zurück, unterscheiden sich Maschinenbürokratien von professionellen Bürokratien dadurch, daß die Anwesenheit von Beschäftigungskennzeichen des intemen Arbeitsmarktes bei Maschinenbürokratien deutlich stärker ausgeprägt ist als vergleichsweise bei den professionellen Bürokratien.

Neben einem Vorrat an gemeinsamen Beschäftigungskennzeichen bestehen unterschiedliche Beschäftigungskennzeichen, die es aus der Sicht interner Arbeitsmärkte rechtfertigen, von eigenständigen Beschäftigungsorganisationen zu sprechen, in denen spezifische betriebliche Beschäftigungsprofile bzw. -kulturen bestehen. Die ErschlieBung der betrieblichen Beschäftigungssituation wird aus der Sicht. der Organisationskonfigurationen konkretisiert und systematisiert. Emöglicht werden neben Diagnosen und Prognosen der Personalentwicklung Entscheidungshilfen für die bewuBte Gestaltung des betrieblichen Arbeitsmarktes.

\subsubsection{Implikationen für das Personalmanagement}

Zwei Aspekte stehen in diesem Abschnitt im Vordergrund. Zum einen die Rückkoppelung zu verschiedenen Konzeptionen des Personalmanagements, zum anderen die praktischen Implikationen der Ergebnisse dieser Untersuchung.

Die Untersuchung hat durch die verschiedenen Analyseniveaus aufgezeigt, wo die Ansatzpunkte für das Personalmanagement liegen. Bei den untersuchten Organisationen war die Mobilität meist Folge einer frei werdenden Stelle. Die Reallokation bot Anlaß für Aktionen des Personalmanagements (Atkinson, 1984). Demgegenüber steht die interne Karriereplanung und -entwicklung (Hall, 1986; v. Maanen, 1977), wie sie in einem Managementdevelopmentsystem zum Ausdruck kommen kann. Solche Instrumente waren bei den untersuchten Organisationen nur in Ansätzen anzutreffen. Dabci spielt die strategische Personalentwicklung (Rieckmann, 1989; Sadowski/Frick, 1989; Thom, 1987) eine Rolle. Die Wahrscheinlichkeit, eine solche anzutreffen, steigt mit einer größeren Beschäftigtenzahl. 
Diese Studie hat die analytischen Strukturen (Kalleberg, 1988; 1989; Kanter, 1984; Preisendörfer, 1989) beschrieben und die Unterschiede im Kontexı der jeweiligen Organisationskonfiguration herausgearbeitet. Damit kann nun umgekehrt der Weg der Synthetisierung organisatorischer Allokationsbedingungen beschritten werden. Es geht dabei um die bewußte Gestaltung von intemen Arbeitsmärkten, bei der die Erkenntnisse dieser Arbeit gestalterisch umgesetzt werden können. Ansätze dazu sind vorhanden (Hoof, van, 1991). Sie bewegen sich im Rahmen des Systemdenkens (de Sitter/den Hertog, 1990; Scholz, 1989; Rowbottom/Bills, 1987; Domsch/Schneble, 1987; Grochla, 1980; Luhmann, 1975). Für das Personalmanagement stellt sich das Problem der Abstimmung unterschiedlicher Teile des 'Systems' betrieblicher Beschäftigung unter Berücksichtigung der ökonomischen Entwicklung (Kluytmans, 1990). Die herrschenden Personalmanagementvisionen (Semlinger, 1989) begünstigen die Verbreitung von Human Resource Management (Hendry/Pettigrew, 1990) inklusiv des strategischen Bezugs. Die utilitaristische Sichtweise (Tomer, 1987), wobei 'der Mensch im Mittelpunkt steht bzw. der Mensch Mittel ist' (Neuberger u. a., 1990), ist unverkennbar. Eine organisationsbezogene Sichtweise kann dabei eine Alternative repräsentieren (Sievers, 1988).

Zu den praktischen Implikationen für das Personalmanagement lassen sich die folgenden Erkenntnisse rechnen.

Aufgrund der Tendenz zur 'Alterslastigkeit' des Personals in Maschinenbürokratien empfiehlt es sich, die demographische Entwicklung des Personals permanent zu beobachten und durch entsprechende Rekrutierungspolitik die Nachteile einer solchen Entwicklung aufzufangen.

Die aufgezeigten Beschäftigungsstrukturen bilden die Ansatzpunkte einer aktiven Personalpolitik unter bewußter Gestaltung betrieblicher Beschäftigung. Dazu gehört insbesondere die diagnostische Einschätzung und Berücksichtigung organisationsspezifischer Beschäftigungsbedingungen.

Soweit der inteme Arbeitsmarkt als geeignete Beschäftigungsform gesehen wird, gilt es, partizipativ die Beschäftigten in die Gestaltung betrieblicher Beschäftigung einzubeziehen. Damit läßt sich 'commitment' in Form einer Organisationskultur aufbauen als Gegenprinzip zum hierarchischen Kontrollprinzip.

Zur Vermeidung der Nachteile eines zu langen Verbleibs auf 'dead end positions' von Führungskräften im 'middle line-Bereich' ist eine funktionale Flexibilisierung an Endpositionen von Funktionsketten anzustreben, die auf eine gruppenbezogene Zusammenarbeit/ Koordination hinausläuft unter Ausschaltung einer toumament-Allokation.

$\mathrm{Zu}$ einer bewußten Personalpolitik in intemen Arbeitsmärkten gehört eine kontinuierliche kritische Bewertung der Folgen zunehmender Institutionalisierung und Segmentierung der betrieblichen Beschäftigung und der daraus entstehenden Diskriminierung von Teilen des Personals.

Auf diesen letztgenannten Aspekt gilt es, im folgenden Abschnitt näher einzugehen. 


\subsection{Segmentierung des betrieblichen Arbeitsmarktes}

Die im vorhergehenden Abschnitt konstatierten Erkenntnisse bezüglich interner Allokationsgrenzen erfordern eine Neubewertung der Segmentationsproblematik betrieblicher Arbeitsmärkte (Schwan/Soeters, 1991c). Statt der von Piore (1975) entwickelten Einteilung betrieblicher Arbeitsmärkte in drei Segmente konnte mit dieser Arbeit der Nachweis erbracht werden, daß bei den untersuchten Organisationen intraorganisationale Mobilitätsmuster bestehen, die entsprechend der Mintzbergschen 'Parteinteilung' eine Segmentation des betrieblichen Arbeitsmarktes in fünf 'Segmente' nahelegen. Die Rigidität innerorganisatorischer Partgrenzen hinsichtlich der Mobilitätsdurchlässigkeit bestätigt diese Einteilung. In der Literatur hat es bis in jüngster Zeit Versuche gegeben, zu einer differenzierteren Sichtweise beizutragen (Loveridge/Mok, 1979; Lutz/Sengenberger, 1974; Lulofs, 1987).

Ausgangspunkt war ein dualökonomisch geprägtes, volkswirtschaftliches Denken und die Suche nach Formen der Arbeitsdiskriminienung (Averit,, 1968; Lee/Loveridge, 1987). Die in dieser Arbeit benutzte 'Parteinteilung' basiert erstmals auf einer organisationsanalytischen Betrachtung betrieblicher Beschäftigungsformen sowie aufgrund von Mobilitätsdynamik innerhalb der Segmente. Für Maschinenbürokratien stand bislang die Sichtweise zentraler 'ports of entry' im Mittelpunkt, Diese Perspektive muß relativiert werden. Die 'ports of entry' der untersuchten Maschinenbürokratien sind danach verteilt auf die unteren Funktionshierarchien 'operating core', 'technostructure', und 'support staff' und nicht konzentriert auf ein zentrales 'Eintrittsniveau'. Ein Unterschied zwischen Maschinenbürokratien und professionellen Bürokratien kommt dadurch zum Ausdruck, daß bei Maschinenbürokratien die 'ports of entry' auf untere Hierarchieniveaus (mit Ausnahme des part 'strategic apex') konzentriert sind, während bei professionellen Bürokratien der Zugang grundsätzlich auf allen Hierarchieniveaus erfolgen kann. Aus der Empirie betrieblicher Beschäftigung eröffnet sich damit eine neue Sichtweise der Segmentationsproblematik.

Eine ebenfalls in der Literatur anzutreffende Tendenz zur 'Multisegmentierung' des Arbeitsmarktes (Biller, 1989; Sengenberger, 1987; Loveridge/Mok, 1979) hat ihre Rechtfertigung, wenn man die Segmentationsperspektive ausweitet auf Einteilungen innerhalb der Segmente. Die Logik dieser Einteilung legt nahe, daß neben Segmenten auch von 'Subsegmenten' die Rede sein kann. Die empirischen Befunde dieser Arbeit zeigen auf, daß eine weitergehende 'Segmentierung' des betrieblichen Arbeitsmarktes besteht, die in dieser Arbeit in Form der Konzeption der Abteilungsgrenzen, Funktionsketten und Berufsgruppen allokationsrelevant zum Ausdruck kommt (Biehler u. a., 1981). Im Rahmen des Abschnitts 'Theorierückkoppelung' wird auf die Funktion der Segmentation von betrieblichen Arbeitsmärkten in einem weiteren Aspekt eingangen.

\subsection{Rückschlüsse auf die Beschäftigungsstrategie}

Die Ergebnisse der Untersuchung repräsentieren Einzelphänomene, die im jeweiligen organisatorischen Kontext als nicht voneinander unabhängig zu sehen sind. Die Beschäftigungsstrategie wird als Teil der Organisationsstrategie (Romme, 1992) gesehen. Die Resultate der Untersuchung bilden Indikatoren, die Rückschlüsse auf die Beschäftigungsstrategie ermöglichen. Zugrunde liegt die Erkenntnis, daß aus der Strategie die Struktur 
folgt (Chandler, 1962) oder auch umgekehr (Mintzberg, 1990). Auf konkreterem Niveau bedeutet dies, daß "...elements of structure cohere within common configurations, as do those of strategy. ... The configurations ... are said to be predictively useful in that they are composed of tight constellations of mutually supportive elements. The presence of certain elements can thus lead to the reliable prediction of the remaining elements ..." (Miller, 1986, S. 235 f.).

Romme u. a. (1990) sprechen der 'strategischen Logik der Aktion', die zur Entscheidungsfindung und strategischem Handeln beiträgt bis "...there is a pattern in a stream of decisions and actions that becomes an overall strategy followed by the organization", (Romme u. a., 1990, S. 47; Mintzberg/Waters, 1985).

Bezieht man dic empirischen Ergebnisse dieser Studie im genannten Sinne auf die Beschäftigungskriterien, die im Zusammenhang mit den Organisationskonfigurationen stehen bzw. aus der Literatur ableitbar sind (Gegenüberstellung der beiden Organisationskonfiguration in Kapital 3), dann zeigt sich ein weitgehend konsistentes Bild. Die empirisch ermittelten Elemente der Beschäftigung bestätigen die 'strategische Logik der Aktion', die für die jeweilige Organisationskonfiguration im Zeitablauf und durch entsprechende Handlungen zutrifft.

Im Rahmen dieser Untersuchung wurden strukturelle (Kapitel 2 u. 3) und zeitliche (im Rahmen der Beschäftigungszeitanalyse, Kapitel 7) Strategieelemente identifiziert. Angesichts der zunchmenden Bedeutung der Personalmanagements und dessen zunehmender Integration in ein Konzept des stategischen Managements (Elsik, 1992; Butler et al. 1991; Scholz, 1987; Pennings, 1985) stellt sich die Frage, welche der hier dargestellten Organisutionskonfigurationen mit ihren Unterschiedlichkeiten zukünftig im Rahmen des strategischen Managements mehr Aufmerksamkeit verdient und bei der Gestaltung von betrieblichen Beschäftigungssystemen größere Implementierungschancen besitzt, vorausgesetzt, die Auswahl ist auf die beiden Organisationskonfigurationen beschränkt.

Dabei geht es um die Frage einer verstärkten Durchsetzung interner Arbeitsmärkte mit zunehmender interner Dynamik, verschiedenen Formen intemer organisatorischer Mobilitätsgrenzen, aber auch um die Herausbildung von 'Besitzständen' der Beschäftigten (Property Rights; Balzer, 1987) im Rahmen der Betriebsgemeinschaft. Das institutionelle Moment (Backhaus, 1980, 1982) wird verstärkt durch bürokratische Merkmale, aber auch durch Auflagen des Gesetzgebers. Brandes/Buttler (1989) sprechen von der 'Unvermeidbarkeit' interner Arbeitsmärkte aufgrund des permanenten Koordinationsbedarfs bei der Allokation von Arbeit.

Mit den professionellen Bürokratien steht eine mehr offene Organisationskonfiguration mit mehr professionellen Anforderungen, aber auch mit einer stärkeren Individualisierung der Beschäftigten bis hin zum 'Intrapreneur' im Vordergrund. Es liegt in der Entwicklungslogik dieser Organisationskonfiguration, daß das PTinzip der Risikoübemahme das Prinzip der 'Arbeitgeberfürsorge' ablöst. Der Agent wird zum Prinzipal bzw. wird selbständig. Besonders bei der Wirtschaftsprüfungsgesellschaft ist dieser potentielle Karriereschritt in der Funktionskette vorgesehen. 
Die Integration von Organisations- und Beschäftigungsstrategie kommt u. a. zum Ausdruck im Interaktionsmuster mit der Umgebung. Maschinenbürokratien als Ausprägung intemer Arbeitsmärkte bilden gegenüber der Außenwelt eine geschlossene Einheit. Der Zugang von Arbeitnehmem wird durch die 'ports of entry' kontrolliert. Die Allokationsdynamik hängt ab von intemen Regeln, Gewohnheiten und der entspechenden Arbeitsplatzinfrastruktur. Akzentuiert wird hier die Organisations- gegenüber der Marktlösung (Schreuder, 1990; Williamson, 1975). Die professionellen Bürokratien verkörpern mehr die Marktausrichtung. Hinsichtlich der Beschäftigungsstrategie erfordert diese Organisationskonfiguration eine andere Art der Marktdurchdringung zur Erschließung des potentiellen Personals. Unter diesem Aspekt sind die Anforderungen, die an eine 'unternehmensorientierte Arbeitsmarktstrategie' (Scherm, 1991; Scholz, 1989; Drumm, 1989b) zu stellen sind, organisationsspezifisch determiniert. Bei professionellen Bürokratien sind die diesbezüglichen Anforderungen hinsichtlich der Marktdurchdringung höher als bei den Maschinenbürokratien.

\subsection{Theorierückkoppelung}

Ausgangspunkt der empirischen Untersuchung waren die Transaktionskostentheorie und die Kontingenztheorie. Gegenstand dieser Arbeit war nicht die empirische Überprüfung der Theorien. Vielmehr dienten sie als analytischer Zugang zur Konkretisierung der zentralen Fragestellung. Im folgenden konzentriert sich die Betrachtung daher auf die Frage, in welcher Art die Ergebnisse der Untersuchung zu einem besseren Verständnis der betrieblichen Organisation von Arbeit bzw. der Arbeitsorganisation im Licht der beiden Theorien beitragen.

\subsubsection{Rückkoppelung zur Transaktionskostentheorie}

Für die Rückkoppelung der Befunde dieser Untersuchung zur Transaktionskostentheorie greifen wir auf das analytische Schema von Douma/Schreuder (1991) zurück. Die ökonomische Bedeutung der Transaktionskostentheorie soll auf den verschiedenen allokationsrelevanten Analyseebenen betrachtet werden.

Gemeinsam ist allen Betrachtungsebenen die Abwägung bzw. der Vergleich mit alternativen Transaktionskosten. Dieses 'Prinzip ökonomischer Entscheidungsfindung' ist wiederkehrend und stellt sich auch dann, wenn eine Grundsatzentscheidung zugunsten einer 'Hierarchielösung' (intemer Arbeitsmarkt) (Hendrikse/Schreuder, 1987) statt einer 'Marktlösung' getroffen wurde. Als Beispiel läßt sich die Ptäferenz der internen Rekrutierung aus unmittelbarer Organisationsnähe in Maschinenbürokratien auf der Basis eines guten Informationsniveaus anführen. Wir gehen nun ein auf die verschiedenen Analyseebenen.

Auf der 'Systemebene' ergibt sich im Rahmen dieser Arbeit eine eingeschränkte Sichtweise der Abwägung zwischen Markt und Hierarchie. Dies hängt von dem Faktum ab, daB Organisationen Gegenstand des Erkenntnisinteresses waren. Aus einer differenzierteren Perspektive (Douma/Schreuder, 1991) stellt sich die Wahl zwischen Markt und Hierarchie jedoch emeut. Dabei handelt es sich um organisatorische Märkte, also marktmäßige Koordinationsformen innerhalb von Organisationen. Williamson (1991) unterstreicht die Bedeutung der Hybridform zwischen Markt und Hierarchie. Der interne Arbeitsmarkt kann 
auf diese Weise funktionieren (Douma/Schreuder, 1991), allerdings ergeben sich für die vier untersuchten Organisationen keinerlei Anhaltspunkte für eine vorhandene marktmäßige, d. h. durch die preisliche Allokationssteuerung beeinflußte betriebliche Arbeitsmärkte (Ochsenbauer, 1989).

Auf der Ebene der 'Population von Organisationen' standen die zwei Organisationstypen im Vordergrund. Dabei war der eine Organisationtyp mehr von einer 'intemen Dynamik' hinsichtlich der Arbeitsmarkıallokation beherrscht, verbunden mit einem 'beschränkten' Austausch mit der 'Außenwelt' (dem extemen Arbeitsmarkt). Beim zweiten Organisationstyp war die Allokation von Arbeit als Interaktion mit der Außenwelt deutlich stärker ausgeprägt. Dies bedeutet nicht, daß ein höherer Grad an 'Marktnähe' auch 'marktähnliche Verhältnisse' auf dem betrieblichen Arbeitsmarkt einschließt.

Die 'Analyseebene der Organisation' (Douma/Schreuder, 199l) läßt verschiedene 'governance structures' erkennen, die parallel in einer Organisation bestehen können. Dies war zum einen der 'wechselseitig verpflichtende Markt', eine 'governance structure', die Parallelen aufweist zu den Beschäftigungsmerkmalen des internen Arbeitsmarktes. Zum anderen das 'einfache Team' als die Beschäftigungsform, die mit den professioneilen Bürokratien zu vergleichen ist.

Auf der 'Analyseebene der Gruppe bzw. der Person' steht die Koordination der Beschäftigungsverhältnisse im Vordergrund. Ausgangspunkt ist die Klassifikation transaktionsspezifischer Koordinationserfordernisse. Wir konzentrieren uns dabei auf die relevanten 'governance structures' des 'wechselseitig verpflichtenden Marktes' sowie des 'Einfachen Teams'.

Die Koordinationserfordemisse hinsichtlich der Allokation von Arbeit hängen von der Schwierigkeit ab, die Produktivität des einzelnen Beschäftigten zu ermitteln. Beim 'Einfachen Team' ist die individuelle Produktivitätsmessung schwierig und das 'Humankapital' nicht spezifisch. Um opportunistisches Verhalten bzw. die Rücknahme des Arbeitseinsatzes des Arbeitnehmers zu reduzieren, sind zusätzliche Koordinationsmaßnahmen angebracht in Form von Regelungen wie Senioritätsbezahlung, Koppelung der Löhne an Arbeitsplätze, bestimmte Eintrittspositionen sowie inteme Beförderungen. Die konsequente Umsetzung dieser Regelungen kann zu einer Beschäftigungssituation führen, die nahezu der des internen Arbeitsmarktes entspricht.

Bei der 'govemance structure' des 'wechselseitig verpflichtenden Marktes' ist die Messung der Produktivität leichter, jedoch das Arbeitsvermögen spezifisch. Eine wechselseitige Bindung zwischen Arbeitnehmer und Arbeitgeber im Rahmen einer 'protective governance structure' (Williamson, 1984) kann 'irrationalen' Verhaltensäußerungen entgegenwirken. Die Koordinationsmaßnahmen sind hier im Sinne des internen Arbeitsmarktes in der Definition von Lutz (1987) entwickelt und damit stärker ausgeprägt als bei der Form des 'Einfachen Teams'.

Der in Form von formellen Institutionen und informellen Normen zum Ausdruck kommende beschäftigungsrelevante Koordinationsbedarf bei internen Arbeitsmärkten deutet auf eine effiziente Form betrieblicher Beschäftigung hin. Dennoch ist die unternehmens- 
interne Koordination der Allokation von Arbeit nicht vermeidbar (Brandes/Buttler, 1989). Ansatzpunkte für Allokationsintervention kommen zum Ausdruck in der kaskadenförmigen Organisationsstruktur, die im theoretischen Teil der Arbeit aus der Arbeit von Doeringer/ Piore (1971) rekonstruiert wurde, aber auch in Form der für empirische Zwecke reduzierten Form. Macht (Turk, 1983; Mintzberg, 1983) sowie Konzepte wie Atmosphäre (Spangenberg, 1989) bzw. Organisationskultur (Hofstede, 1991; Heinen, 1987) wirken in Richtung 'consummate cooperation', also Arbeitnehmerverhalten, das eine höhere Produktivität erwarten läßt durch Beschäftigungsstabilität.

Die Untersuchung lenkt die Aufmerksamkeit auf zwei sich ergänzende institutionelle Prinzipien der Funktionsweise interner Arbeitsmärkte. Zum einen geht es um die Vielzahl der Institutionen der Beschäftigung, die durch Regeln bzw. einem impliziten Vertrag zum Ausdruck kommen. Zum anderen geht es um ein auf Beherrschung der internen Organisation gerichtetes Organisationsstrukturprinzip, nach dem die Allokation in Beschäftigungseinheiten einschließlich definienter Grenzen ermöglicht wird. Ein organisationstheoretisches 'Gegenstïck' zur Segmentationstheorie findet sich in Scotts (1987) Überlegungen zu den organisatorischen Grenzen intemer Arbeitsmärkte, im theoretischen Teil der Arbeit als 'Organisationsrahmenkonzept' bezeichnet.

\subsubsection{Rückkoppelung zur Kontingenztheorie}

Obwohl Arbeitsorganisationen ihre eigenen Akzente haben, konnte im Rahmen dieser Arbeit der Nachweis erbracht werden, daß Mintzbergs analytisches Instrumentarium einen heuristischen Wert aufweist in Bezug auf Arbeitsorganisationen. Für die mikroorganisatorische anaiytische Durchdringung der Arteitsorganisationen waren ergänzende Konzeptionen erforderlich. Mintzbergs Perspektive zur Erschließung der Organisationsstruktur hinsichtlich der Allokation von Arbeit endet auf dem Niveau der Parts. Weitergehende allokationsrelevante Organisationsstrukturen in dieser Hinsicht sucht man vergebens. Mintzbergs analytisches Rahmenwerk bietet einen Einstieg und empirischen Zugang. Zur Erschließung der mikroorganisatorischen Allokationswelt bedarf es, wie die Arbeit gezeigt hat, ergänzender Konzepte.

Interme Arbeitsmärkte weisen Beschäftigungsmerkmale auf, die mit den Organisationscharakteristiken Übereinstimmungen zeigen. Werden professionelle Bürokratien mit intemen Arbeitsmärkten verglichen, dann zeigen sich Übereinstimmungen hinsichtlich bestimmter Merkmale.

\subsection{Weiterführende Untersuchungsperspektiven}

Das erhobene Datenmaterial läßt vom Umfang und von der Qualität weitere Analysen zu. Im Rahmen dieser Studie konnte deshalb nur ein Teil der Informationen verarbeitet werden gemessen an den Möglichkeiten, die potentiell zur Verfügung stehen. $\mathrm{Zu}$ den weiterführenden Untersuchungsperspektiven sind die folgenden Forschungsfelder zu rechnen. 
Die mikroorganisationale Bestimmung der Allokation aus organisationsstruktureller Sichtweise. In Kapitel 6 wurde auf die unterschiedliche Gestaltung der Mobilitätsketten eingangen. Hier zeigt sich eine Komplexitäl, deren Rationalität es zu untersuchen gilt.

Ein weiterer Aspekt konzentriert sich auf die Organisationsgrenzen der intraorganisationalen Allokation. $\mathrm{Zu}$ fragen ist, wie durchlässig können diese Grenzen sein bzw. unter welchen Bedingungen vollzieht sich eine Öffnung bzw. Schließung der Organisationsgrenzen (Endruweit, 1988). Welches sind die dominanten bzw. weniger dominanten Grenzen für die Allokation? Die in dieser Arbeit analytisch erschlossenen Grenzen nach Doeringer/Piore (1971) sowie die im Rahmen der offenen Stellenanalyse für empirische Zwecke reduzierte Konzeption sagt noch wenig über die Permeabilität durch Arbeitnehmermobilität.

Ein anderer Aspekt betrifft die weitere Erschließung der Hierarchiedimension. Zu fragen wäre, inwieweit besteht ein für die jeweilige Organisationskonfiguration typische Kontrollspanne, die im Rahmen welcher Beschäftigungseinheiten anzutreffen ist. Hier kann sich eine neue Dirnension der mikroorganisationalen Beschäftigung ergeben. Althauser/Kalleberg (1990, S. 325) sprechen in diesem Zusammenhang von 'vertical lattice of positions'. Dabei wird der Akzent auf organisationsstrukturelle Kennzeichen gelegt, aber auch auf den Faktor Zeit.

In dieser Studie ist die Rede von bürokratischen Organisationen. Bürokratie wird dabei mehr aufgefaßt als Maßstab für einen hohen Grad an Institutionalisierung von betrieblicher Beschäftigung. Weitgehend ausgeklammert bleibt die in der organisationssoziologischen Tradition stehende, oft vergessene Bürokratietheorie Webers sowie aktuellerer Repräsentanten wie Gouldner (1964), Selznick (1965) und Mayntz (1968). In Bezug auf den intemen Arbeitsmarkt stehen zwei Aspekte im Mittelpunkt: zum einen die Funktion der Bürokratie hinsichtlich der Beherrschung von Beschäftigung innerhalb von Organisationen (DiPrete, 1989; Hondeghem, 1990) mit möglichen Kennzeichen eines institutionellen Äquivalents (durch Regelgebung) zur Preisfunktion am Markt, zum anderen die Frage, inwiewcit durch bürokratische Strukturen ein 'Selbststeuerungsprinzip' von Organisationen etabliert wird, das neben Nachteilen (das Einschleusen von Personen besonderer Präferenz unter Umgehung allgemein festgelegter Allokationsregeln) auch deutliche ökonomische Vorteile aufweist.

Schließlich bleibt ein Aspekt, nämlich der des Funktionierens intemer Arbeitsmärkte unter verschiedenen nationalen Kultureinflüssen. Beschäftigungsmuster wie sie in dieser Arbeit aufgezeigt wurden, müssen nicht unbedingt übereinstimmen mit denen der Vereinigten Staaten oder Japan in vergleichbaren Organisationen. Auf Grund von unterschiedlichen Beschäftigungskulturen (Osterman, 1988) liegt es nahe, zu überprüfen, inwieweit unter anderen Kultureinflüssen andere Beschäftigungsmuster zustande kommen bzw. worauf dies zurückzuführen ist.

\subsection{Interne Arbeitsmärkte im gesellschaftlichen Kontext}

In diesem Abschnitt sollen Perspektiven der Zukunft der Arbeit angesprochen werden und die Rolle, die interne Arbeitsmärkte dabei ausfüllen. 
Über Anpassungen am Arbeitsmarkt sind Entwicklungen aufzufangen, die im Zeitablauf eine Veränderung des "arbeitsmarktpolitischen Gleichgewichts" bewirken. Die langfristige Entwicklung am Arbeitsmarkt verdient Aufmerksamkeit. Interne Arbeitsmärkte spielen dabei eine entscheidende Rolle. Bei einer zunehmenden Selektivität der Erwerbschancen bilden inteme Arbeitsmärkte den Zugang zu qualitativer Beschäftigung, d. h. Karrierechancen, während andere Beschäftigungsformen eine geringere Qualität aufweisen, ähnlich den in der dualen Arbeitsmarkttheorie unterstellten Wirkungen. Interne Arbeitsmärkte bilden die institutionellen Lösungen im Zuge einer ungleichmäßigen Verteilung von Beschäftigungschancen (soziale Schließung). Unterschiedliche Facetten der sozialen Schließung prägen die zukünftige Entwicklung der Arbeit (Arendt, 1981). Einige sollen hier kurz skizziert werden.

Die Technikentwicklung (Brödner, 1985; Sorge, 1985 u. 1987) im Produktionsbereich zeigt Tendenzen, die aufgrund von Automatisierung andere Anforderungen an menschliche Arbeitskraft (Bosch, 1986) erfordem. Kem/Schumann (1984) konstatieren das 'Ende der Arbeitsteilung', doch ist dies keineswegs eine Schlüsselkategorie für mehr menschlichen Arbeitseinsatz. Vielmehr wird durch diese Tendenz eine Veränderung des Qualifikationspotentials ausgedrückt, die kaum eine Breitenwirkung haben wird. Piore/Sabel (1985) gehen von einem Abbau 'alter mechanischer Organisationen' aus. Dabei wird die Hierarchie nicht mehr die entscheidende Rolle spielen. Angesagt ist die 'flexible Spezialisierung' von Produktionsstrukturen.

In den Niederlanden (Windmuller/de Galan/Zweeden (1987) ergeben sich Anzeichen für eine Zunahme der 'Outplacementkultur' (Mayrhofer, 1989), eine 'kultivierte' Form der Trennung der Mitarbeiter von der Organisation, durch eine steigende Zahl von Beratungsunternehmen, die diese Dienstleistung anbieten. Außerdem erwächst aus dem allgemeinen Trend der Reduzierung staatlicher Aktivitäten zugunsten von 'Marktaktivitäten' eine 'liberalere' Handhabung des Arbeitsvermittlungsmonopols, das bisher von staatlicher Seite wahrgenommen wurde. Dadurch bekommen Arbeitnehmeruberlassungsunternehmen mehr Möglichkeiten der Arbeitsvermittlung.

Der interne Arbeitsmarkı dürfte im Lichte dieser Entwicklungen eine Funktion als 'Rückhaltebecken' der Beschäftigung einnehmen, d. h. Sicherung der 'property rights' der Kernarbeitnehmer (Kluytmans/Paauwe, 1991), jedoch 'flexibel' hinsichtlich der Gruppe der Arbeitnehmer, die noch keine 'Besitzstände' aufgebaut haben. Auf Grund der Allokationsgrenzen innerhalb der Organisation des internen Arbeitsmarktes ist eine Beeinflussung im Rahmen der Arbeitsmarktpolitik nur beschränkt möglich. Staatliche Interventionen auf Grund zu hoher Arbeitslosigkeit sind vielfach von einer neoklassischen bzw. keynesianischen Denkrationalität geprägt, die die Komplexität und Dynamik intemer Arbeitsmärkte weitgehend unberücksichtigı läßt. Dadurch verkommen beschäftigungsstabilisierende Maßnahmen vielfach zu Mitnahmeeffekten, obne daß die Logik des Funktionierens intemer Arbeitsmärkte zum Gegenstand beschäftigungspolitischer Gestaltung wird.

Weitere Überlegungen sind im sozialphilosophischen Bereich anzusiedeln. So fragt Jahoda (1983): wieviel Arbeit braucht der Mensch? Guggenberger (1988) wählt eine andere Perspektive, die aus der Diskussion um die Verkürzung der Arbeitszeit resultiert. Zentral steht die Überlegung, daß langfristig der Gesellschaft die Arbeit ausgeht, weil man sich 
u. a. einer Technik bedient, die menschliche Arbeitskraft immer weniger zum Gegenstand gesellschaftlicher Verantwortung macht.

Empirische Fragestellungen repräsentieren einen Ausschnitt aus der Wirklichkeit. Darin liegt eine Beschränkung, die dazu beiträgt, daß die dahinterstehende ökonomische Rationalität in der Betrachtung erst beim zweiten Blick erfaßt wird. Inteme Arbeitsmärkte sind nicht unabhängig von der gegenwärtigen ökonomischen Rationalität zu sehen.

Hinsichtlich der Beschäftigung in Form intemer Arbeitsmärkte gilt es zu fragen, welche Rolle diese im Rahmen einer zukünfigen Gesellschaft spielen werden. Zwei altemative Entwicklungen stehen dabei zur Diskussion. Unter Fortschreibung der ökonomischen Rationalität (Ulrich, 1986) stellt der interne Arbeitsmarkt ein 'Beschäftigungsrefugium' dar, das in der Tendenz immer weniger Menschen Beschäftigung bietet. Die andere Entwicklung betont die gesellschaftliche Verantwortung, baut auf 'Integration' der ökonomisch und sozial Schwächeren und ermöglicht die Abkehr von der Priorität ausschließlich ökonomischer Rationalităt. Abschließend kann dies als Vision ausgedrückt werden, deren Entwicklungsgrundlagen in der Gegenwart liegen. Gorz drückt diese Vision als Übergang aus "... von der Arbeitsgesellschaft zu einer Gesellschaft der befreiten Zeit, in der Kultur und Gesellschaftlichkeit das Ökonomische überwiegen - es geht um eine 'Kulturgesellschaft"" (Gorz, 1989, S. 257). 


\section{Samenvatting (Zusammenfassung in niederländischer Sprache)}

\section{Organisatieconfiguraties en interne arbeidsmarkten}

Een theoretisch-empirisch onderzoek naar werkorganisaties in het licht van interne arbeidsmarkten

Deze dissertatie bevat een onderzoek naar het functioneren van interne arbeidsmarkten binnen vier verschillende ondememingen/instellingen (een ziekenhuis, accountantskantoor, productieondememing en sociale verzekeringsinstelling). Voor de definiëring van organisatieconfiguraties sluiten wij aan bij de benadering van Mintzberg. De eerste twee organisaties vertegenwoordigen de professionele bureaucratie, de twee laatstgenoemde dragen de organisatiekenmerken van de machine-bureaucratie. Het theoretische vertrekpunt bestaat uit een arbeidsmarkttheoretische benadering op basis van de theorie van interne arbeidsmarkten met name de institutionele onderzoekstraditie in de economie. Uit een organisatietheoretische benadering staan twee benaderingen van interne arbeidsmarkten centraal. Dat zijn de transactiekostentheorie van Williamson en de contingentietheoretische benadering.

De centrale vraagstelling is: In welke mate zijn er verschillen tussen machine-bureaucratieën en professionele bureaucratieën in de aanwezigheid van interne arbeidsmarkt kenmerken?

Deze studie bestaat uit twee delen. In het theoretisch gedeelte van deze studie wordt ingegaan op de theorie van de inteme arbeidsmarkt. Afgeleid wordt de analytische structuur van inteme arbeidsmarkten. Deze wordt gerelateerd aan organisatiestructuren afgeleid uit organisatietheoretisch getinte invalshoeken.

Het empirisch gedeelte bestaat uit twee delen: ten eerste een infrastructuuronderzoek van inteme arbeidsmarkten en ten tweede een analyse van vacatures binnen de boven genoemde organisaties. Onderdeel van het infrastructuuronderzoek is een bevorderings-, een salarisontwikkelings- en een analyse van lengte van de diensttijd van personeel binnen de organisaties. De vacatureanalyse omvat een interactieanalyse met betrekking tot vacature vervulling uit in- en externe recruteringsbronnen. Een verdere onderdeel van deze analyse heeft betrekking op inteme recruteringspreferenties binnen de organisatie.

De onderzoeksopzet met betrekking tot infrastructuur van interne arbeidsmarkten en vacature vervulling is gebaseerd op kwantitatief datamateriaal van 306 aselect geselecteerde werknemers (steekproef) verdeeld over vier organisaties en rond 1000 vacatures over een tijdperk van drie jaren. Daarnaast werden meerdere interviews met een gestructureerd 
karakter als kwalitatieve aanvulling afgenomen. De resultaten worden organisatievergelijkenderwijs beschouwd.

Op basis van de empirische gegevens zijn de volgende resultaten m.b.t. de mobiliteitsanalyse te vermelden.

In machine-bureaucratieën vinden meer interne bevorderingen plaats dan bij professionele bureaucratieën. Bevorderingen zijn afhankelijk van leeftijd en bedrijfsspecifieke vaardigheden.

Meestal bestaat op interne arbeidsmarkten een koppeling tussen de salaris- en functiestructuur. Deze analyse bevestigt dat ook in dit opzicht in machine-bureaucratieën een sterkere binding van werknemers optreedt dan in professionele bureaucratieën.

De bedrijfsspecifieke verblijfsduur van werknemers correleert met hoge functieniveaus. De kans op verloop is het grootst aan het begin van de loopbaan.

Concluderend kenmerkt zich de interne arbeidsmarkt door organisatiekenmerken, die overeen komen met de machine-bureaucratie. Maar ook professionele bureaucatieën vertonen karakteristieken van inteme arbeidsmarkten maar voldoen slechts in beperkte mate geheel aan de eisen van de interne arbeidsmarkt.

De vacature-analyse laat zien dat er een interactie plaats vindt tussen vacature-vervulling van buiten (dus externe arbeidsmarkt) en van binnen. Bij machine-bureaucratieën ligt de nadruk op interne vervulling van vacatures; voor een klein gedeelte wordt extem gerecruteerd. Bij professionele bureaucratieën is dit tegengesteld, dus meer vacature vervulling van buiten en weinig van binnen.

Een nadere analyse van vacatures die van binnen vervuld worden maakt duidelijk dat in machine-bureaucatieën personen die van de zelfde afdeling en uit dezelfde functieketen afkomstig zijn met voorrang in aanmerking komen. Inteme recrutering vindt plaats uil de onmiddellijke interne organisatieomgeving. In het geval van professionele bureaucatieën gaat de voorkeur uit naar een andere afdeling maar wel de zelfde functieketen. Dit heeft tot gevolg dat binnen de organisatie grenzen bestaan, die een drempel vormen voor de allocatie. Deze grenzen bieden ook een kans voor het beheren van complexe organisaties. Het onderzoek levert een bijdrage aan de integratie van bedrijfsspecifieke arbeidsmarkten en human resource management uit organisatie perspectief. 


\title{
Summary \\ (Zusammenfassung in englischer Sprache)
}

\section{Organization configurations and internal labor markets}

\begin{abstract}
A theoretical-empirical study of work organizations in the light of internal labor markets
\end{abstract}

The study is focused on the functioning of internal labor markets within four different organizations (hospital, accounting firm, production plant and social security office). We use the Mintzberg view in defining the organizational configurations. The first two organizations represent professional bureaucracies, the other two machine bureaucracies. The theoretical basis is the theory of intemal labor markets which follows the institutional theory tradition and organizational theories of internal labor markets based on the transaction cost approach and the contingency theory.

The central issue of the study is: what kind of differences do exist between machine bureaucracies and professional bureaucracies with respect to the presence of characteristics of internal labor markets?

The study consists of two parts. On the one hand it uses the theory of internal labor markets, and on the other hand it is the organizational theory. The two theoretical parts served as a basis for the derivation of an employment structure.

The empirical study consists of two analysis: an infrastructure analysis of internal labor markets and a vacancy filling analyses. Part of the infrastructure analysis are different subanalyses such as promotion, salary development, and a tenure analysis. The vacancy analysis consists of an interaction analysis of vancancy filling from internal and external recruitment sources. Furthermore the preferences of vacancy filling from intemal sources were subject of a more sophisticated analysis.

One subject of the investigation was a vacancy analysis of four organizations. The four organizations studied were deliberately chosen to represent two different organizational types, according to Mintzberg's typology. As measurement instrument serves a registration form specially developed for the research purpose.

The investigation consisted of the reconstruction and registration according to Mintzberg's analytical organizational framework of all vacancies which have arisen within a threeyears-period. Totally about 1000 vacancies were subject of the analysis. Additionally, we used a questionnaire instrument by means of structured interviews to study the strategic 
logics with reference to the employment relationship in order to obtain qualitativly information.

The results of the infrastructure analysis show that in machine bureaucracies is a more differentiated functional job structure.

Promotions in machine bureaucracies are more often than in professional bureaucracies. The human asset specifity in machine bureaucracies is based on organizational learning on the job. In professional bureaucracies the qualification is a matter of external standardized and extern controlled aquisition. The characteristics of internal labor markets resemble, to a high degree, the occupational characteristics of machine bureaucracies and to low degree to the occupational characteristics of professional bureaucracies.

Internal labor markets are related to a certain wage structure. Also on this level of analysis we confirm that in machine bureaucracies there exist a tendency to bind employees by more mobility dynamic when compared to professional bureaucracies. The organization specific tenure of employees corresponds to a high functional level. High labor turnover mostly occurs in the beginning of the career.

Internal labor markets are highly institutionalized constructs. The vacancy analysis shows an interaction pattern of vacancy filling between the recruitment from the external labor market and from the intemal labor market as a source of hiring personnel. The professional bureaucracies are dominated by extemal vacancy filling. The machine bureaucracies set the priority on internal vacancy filling without neglecting the recruitments from the external labor market.

Additionally the analysis of vacancies filling from inside the organization shows that machine bureaucracies recruit the 'first-preference persons', which come from the same devision and from the same job ladder. Internal recruitment takes place from the direct organizational environment inside the organization. In professional bureaucracies, however, the first recruiting preference tends to other divisions but to the same jobladder. The organizational boundaries form barriers for the allocation. On the one hand the organizational boundaries serve as a management instrument in order to manage the govemance structure of internal labor markets but also as a field of institutionalization and implementation of human resource management from an organizational perspective. 


\section{Anlage zu den Kapiteln 7 und 8}

\section{Kapitel 7}

Tab. 19: Signifikante Pearsonkomelationen der Infrastrukturanalyse (Totalanalyse)

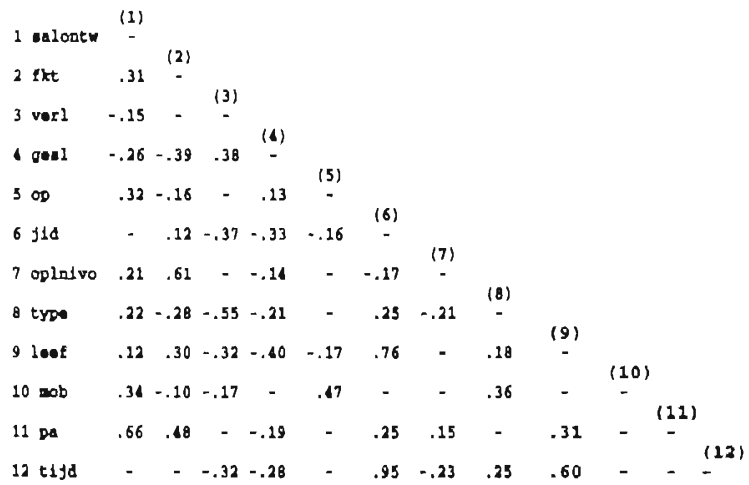

Alle angegebenen Koeffizienten sind signifikant bei $\mathrm{p}<0.05$.

Tab. 20: Signifikante Pearsonkorrelationen der Infrastrukturanalyse (professionelle Bürokratien)

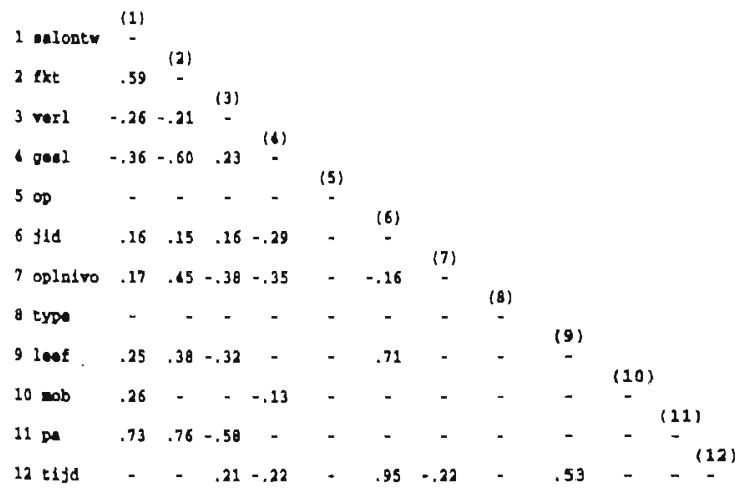

Alle angegebenen Koeffizienten sind signifikant bei $\mathrm{p}<0.05$. 
Tab. 21: Signifikante Pearsonkorrelationen der Infrastrukturanalyse (Maschinenbürokratien)

\begin{tabular}{|c|c|c|c|c|c|c|c|c|c|c|}
\hline 1 ealontw & - & (a) & & & & & & & & \\
\hline $2 \mathrm{fkt}$ & .27 & - & & & & & & & & \\
\hline 3 verI & - & -.18 & - & & & & & & & \\
\hline 4 ges 1 & - & -.34 & .46 & (1) & & & & & & \\
\hline 500 & - & -.63 & .89 & .53 & $\begin{array}{l}(5) \\
-\end{array}$ & & & & & \\
\hline 6510 & - & .25 & -.45 & -.32 & -.66 & (6) & & & & \\
\hline 7 oplativo & .32 & .70 & - & - & - & - & $\begin{array}{l}(7) \\
-\end{array}$ & & & \\
\hline 8 type & - & - & - & - & - & - & - & (8) & (9) & \\
\hline $9 \mathrm{l} \bullet \bullet$ & - & .35 & -.43 & -.37 & -.53 & .78 & - & - & $\cdot$ & \\
\hline $10 \mathrm{mob}$ & .30 & - & - & - & - & - & - & - & -.19 & - \\
\hline 1100 & .57 & .86 & -.32 & -.48 & - & .31 &.$\$ 1$ & - & .49 & -- \\
\hline 12 tijd & -.15 & .18 & -.61 & -.27 & -.62 & .95 & -.17 & - & .63 & - - \\
\hline
\end{tabular}

Alle angegebenen Koeffizienten sind signifikant bei $p<0.05$.

Tab. 23: Beförderungsanalyse: total; Diskriminantenanalyse (MOB als abhängige Variable)

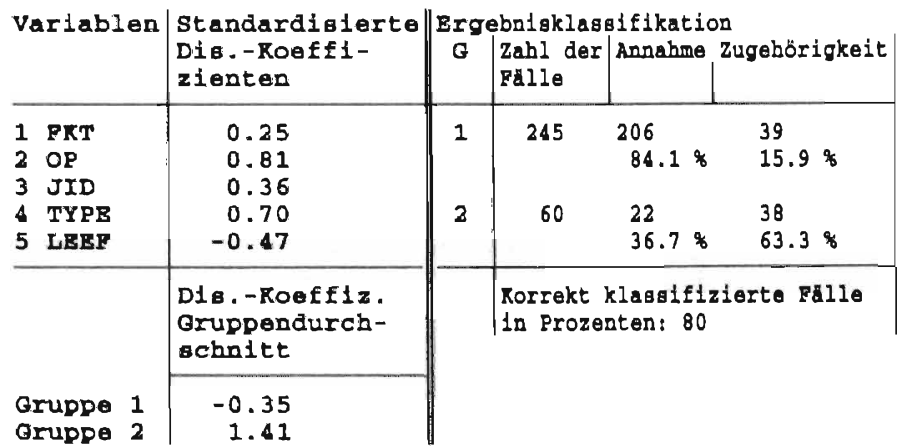

Signifikanz: *** $p<0.001 \quad G=G r u p p e$

D. F. 5

Wilks'Lambda 0.67 N 306 
Tab. 25: Beförderungsanalyse: Maschinenbürokratien; Diskriminantenanalyse (MOB als abhängige Variable)

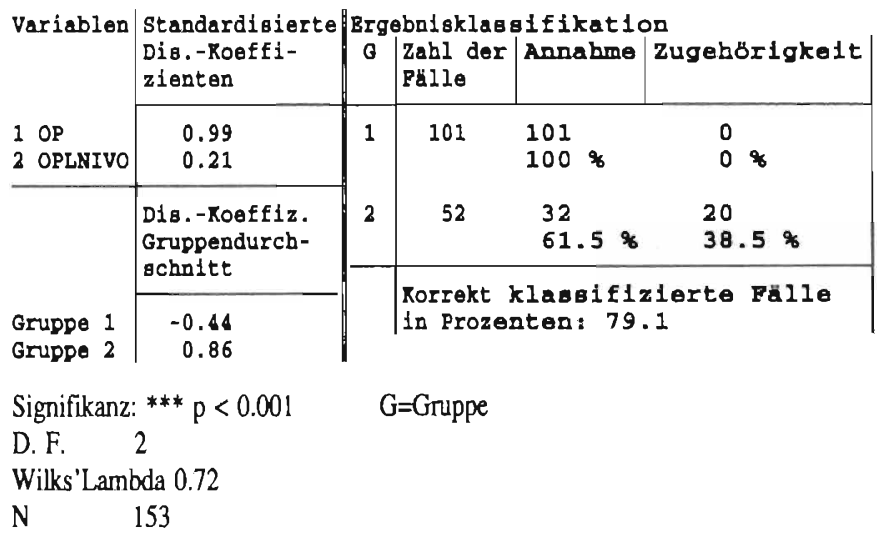

Tab. 33: Breakdown der Variablen: 'Jahre in Dienst' (JID) in Bezug auf die Organisationsteile (parts) der beteiligten Organisationen

\begin{tabular}{|c|c|c|c|}
\hline $\begin{array}{l}\text { Organisation } \\
\text { (Teil/part) }\end{array}$ & $\left|\begin{array}{l}\text { Mittelwert } \\
\text { JID }\end{array}\right|$ & $\begin{array}{l}\text { Standardabwe 1chung } \\
\text { JID }\end{array}$ & Falle \\
\hline $\begin{array}{l}\text { Rrankenhaus } \\
\text { Teil } 1 \\
\text { Teil } 2 \\
\text { Teil } 3 \\
\text { Teil } 4 \\
\text { Teil } 5\end{array}$ & $\begin{array}{r}10.8 \\
6.0 \\
18.4 \\
9.0 \\
8.7 \\
9.9\end{array}$ & $\begin{array}{l}7.7 \\
8.6 \\
6.8 \\
7.0 \\
7.5 \\
6.6\end{array}$ & $\begin{array}{r}75 \\
3 \\
14 \\
20 \\
15 \\
23\end{array}$ \\
\hline $\begin{array}{l}\text { Wirtschaftsprg. } \\
\text { Tell } 1 \\
\text { Teil } 2 \\
\text { Teil } 3 \\
\text { Teil } \\
\text { Teil } 5\end{array}$ & $\begin{array}{r}8.1 \\
14.4 \\
7.7 \\
8.4 \\
16.0 \\
4.4\end{array}$ & $\begin{array}{l}8.2 \\
7.4 \\
5.0 \\
9.1 \\
0 \\
4.9\end{array}$ & $\begin{array}{r}77 \\
10 \\
6 \\
40 \\
2 \\
19\end{array}$ \\
\hline $\begin{array}{l}\text { Sozialversicher. } \\
\text { Tell } 1 \\
\text { Tell } 2 \\
\text { Tell } 3 \\
\text { Tell } 4 \\
\text { Tell } 5\end{array}$ & $\begin{array}{r}9.7 \\
13.0 \\
17.6 \\
7.9 \\
3.5 \\
6.2\end{array}$ & $\begin{array}{l}8.1 \\
9.9 \\
9.3 \\
6.5 \\
3.5 \\
5.6\end{array}$ & $\begin{array}{r}76 \\
2 \\
16 \\
43 \\
2 \\
13\end{array}$ \\
\hline $\begin{array}{l}\text { Produkt lonsuntn. } \\
\text { Tell } 1 \\
\text { Tell } 2 \\
\text { Tell } 3 \\
\text { Teil } 4 \\
\text { Teil } 5\end{array}$ & $\begin{array}{l}18.7 \\
23.0 \\
21.1 \\
14.3 \\
18.2 \\
21.2\end{array}$ & $\begin{array}{l}9.8 \\
15.0 \\
8.1 \\
8.5 \\
11.1 \\
7.9\end{array}$ & $\begin{array}{r}78 \\
4 \\
15 \\
18 \\
24 \\
17\end{array}$ \\
\hline Inegesamt & 11.9 & 9.4 & 306 \\
\hline
\end{tabular}




\section{Kapitel 8}

Tab. 45: Signifikante Pearsonkorrelationen der Vakanzentotal-analyse (Die Abkürzungen kennzeichnen die Variablen)

1 ontrac

2 fkt .06

3 verl -.28

4 verpul $-.89-.05 \quad .30$

5 type

$.36-.24-.66-.37$

Alle angegebenen Koeffizienten sind signifikant bei $p<0.05$.

Tab. 46: Signifikante Pearsonkorrelationen der Vakanzenteilanalyse (Professionelle Bürokratien) (Die Abkürzungen kennzeichnen die Variablen)

\begin{tabular}{|c|c|c|c|}
\hline ontrac & - & (2) & \\
\hline 2 fkt & .06 & - & 131 \\
\hline 3 verl & -.28 & - & - \\
\hline vervul & -.26 & - & - \\
\hline
\end{tabular}

Alle angegebenen Koeffizienten sind signifikant bei $p<0.05$.

Tab. 47: Signifikante Pearsonkorrelationen der Vakanzenteilanalyse (Maschinen bürokratien) (Die Abkürzungen kennzeichnen die Variablen)

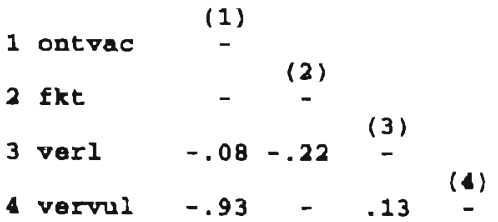

Alle angegebenen Koeffizienten sind signifikant bei $p<0.05$. 
Tab. 55: Signifikante Pearsonkorrelationen der internen Besetzungsanalyse offener Stellen (Die Abkirrzungen kennzeichnen die Variablen)

(1)

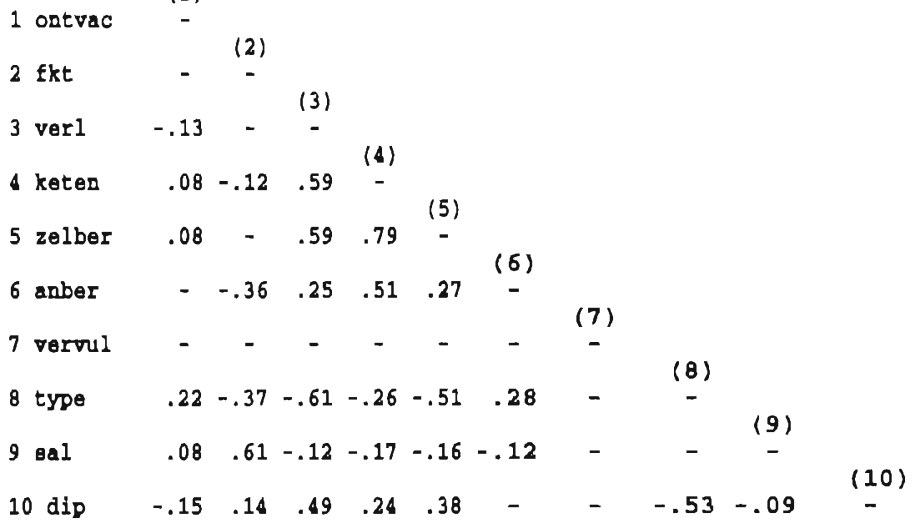

Alle angegebenen Koeffizienten sind signifikant bei $\mathrm{p}<0.05$.

Tab. 56: Signifikante Pearsonkorrelationen der internen Besetzungsanalyse offener Stellen in professionellen Bürokratien (Die Abkürzungen kennzeichnen die Variablen)

(1)

1 ontrac

2 fkt .18 -

3 verl --.39

keter $26 \quad$ (4)

5 zolber (5)

6 (6)

7 vertul $-.16-.20-17$

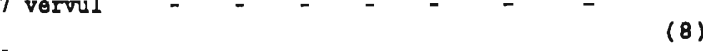

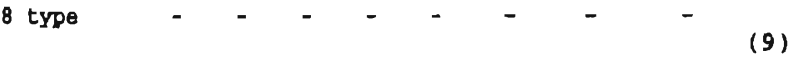

9 sal $\quad .16 \quad .74-.78-.16-.20 \quad-\quad$ - -

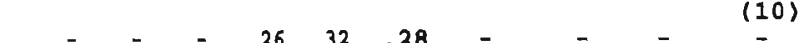

Alle angegebenen Koeffizienten sind signifikant bei $p<0.05$. 
Tab. 57: Signifikante Pearsonkorrelationen der intemen Besetzungsanalyse offener Stellen in Maschinenbürokratien (Die Abkürzungen kennzeichnen die Variablen)

(1)
1 ontrac
2 fkt
$-.09$
3 ver 1
$-\quad-.25$
4 keten
$-\quad-.31 \quad .64$
5 zelber $\quad-\begin{array}{lll}-.28 & .53 \quad .90 & -\end{array}$
6 anber $\quad-\quad-.33 \quad .70 \quad .86 \quad .81^{\prime} \quad$
7 vervul $\quad$ - $\quad$ - $\quad \ldots \quad$ - 17
$\begin{array}{lccccccccccc}7 \text { verval } & - & - & - & - & - & - & - & \\ 8 \text { type } & - & - & - & - & - & - & - & - & \\ 9 \mathrm{gal} & -.10 & .67 & - & -.17 & -.16 & -.15 & - & - & - & \\ 10 \mathrm{dip} & -.13 & - & .26 & .10 & .13 & .16 & - & - & -.09 & -\end{array}$

Alle angegebenen Koeffizienten sind signifikant bei $p<0.05$.

Tab. 49: Besetzung offener Stellen: total; Diskriminantenanalyse (VERVUL als abhängige Variable)

\begin{tabular}{|c|c|c|c|c|c|}
\hline Variablen & $\begin{array}{l}\text { Standardisierte } \\
\text { Dis.-Roeffi- } \\
\text { zienton }\end{array}$ & Brg & $\begin{array}{l}\text { ebnisklas } \\
\text { Zahl der } \\
\text { Palle }\end{array}$ & Annabme & On \\
\hline \multirow[t]{2}{*}{$\begin{array}{ll}1 & \text { ONTVAC } \\
2 & \text { VBRL }\end{array}$} & $\begin{array}{r}0.99 \\
-0.13\end{array}$ & 1 & 535 & $\begin{array}{l}523 \\
97.8 \%\end{array}$ & $\begin{array}{l}12 \\
2.2 \%\end{array}$ \\
\hline & $\begin{array}{l}\text { Dis.-Roeffiz. } \\
\text { Gruppendurch- }\end{array}$ & 2 & 413 & $97.0 \%$ & $\begin{array}{ll}376 \\
91.0 \%\end{array}$ \\
\hline $\begin{array}{ll}\text { Gruppe } 1 \\
\text { Gruppe } 2\end{array}$ & $\begin{array}{r}1.78 \\
-2.31\end{array}$ & & \multicolumn{3}{|c|}{$\begin{array}{l}\text { Rorrekt klassifizierte Palle } \\
\text { In Prozenten: } 94.8\end{array}$} \\
\hline
\end{tabular}
Signifikanz:
$\mathrm{G}=$ Gruppe

D. F. 2

Wilks'Lambda 0.20

N 948 
Tab. 51: Besetzung offener Stellen: professionelle Bürokratien; Diskriminantenanalyse (VERVUL als abhängige Variable)

\begin{tabular}{|c|c|c|c|c|c|}
\hline Variablen & $\begin{array}{l}\text { Standardieierte } \\
\text { Dis.-Roeffi- } \\
\text { zienten }\end{array}$ & $\begin{array}{c}8 r g \\
6\end{array}$ & $\begin{array}{l}\text { ebnisklase } \\
\text { Zahl der } \\
\text { Palle }\end{array}$ & $\begin{array}{l}\text { ifikatic } \\
\text { Annabme }\end{array}$ & zugehörıgkeit \\
\hline \multirow[t]{3}{*}{$\begin{array}{ll}1 & \text { FRT } \\
2 & \text { VERL } \\
3 & \text { ONTVAC }\end{array}$} & $\begin{array}{l}0.17 \\
0.23 \\
0.98\end{array}$ & 1 & 148 & $\begin{array}{r}137 \\
92.6 \%\end{array}$ & $\frac{11}{7.4 \%}$ \\
\hline & \multirow{2}{*}{$\begin{array}{l}\text { Dis. -Roeffiz. } \\
\text { Gruppendurch- } \\
\text { schnitt }\end{array}$} & 2 & 264 & $\begin{array}{r}23 \\
8.7\end{array}$ & $\begin{array}{l}241 \\
91.3 \%\end{array}$ \\
\hline & & & \multirow{2}{*}{\multicolumn{3}{|c|}{$\begin{array}{l}\text { Korrekt klasolfizierte Falle } \\
\text { in Prozenten: } 91.8\end{array}$}} \\
\hline $\begin{array}{ll}\text { Gruppe } & 1 \\
\text { Gruppe } & 2\end{array}$ & $\begin{array}{r}2.01 \\
-1.13\end{array}$ & & & & \\
\hline
\end{tabular}

Signifikanz: *** $p<0.001 \quad G=$ Gruppe

D. F. 3

Wilks'Lambda 0.30

N 412

Tab. 53: Besetzung offener Stellen: Maschinenbürokratien; Diskriminantenanalyse (VERVUL als abhängige Variable)

\begin{tabular}{|c|c|c|c|c|c|}
\hline \multirow[t]{2}{*}{ Vartablen } & \multirow{2}{*}{$\begin{array}{l}\text { Standardisierte } \\
\text { Dis. - Roeffi- } \\
\text { zlenten }\end{array}$} & \multicolumn{2}{|c|}{ Brgebniaklas } & \multicolumn{2}{|c|}{ ifikation } \\
\hline & & & Palle & & $101 \lg k \in 1 L$ \\
\hline \multirow[t]{3}{*}{$\begin{array}{l}1 \text { ONTVAC } \\
2 \text { VBRL }\end{array}$} & $\begin{array}{r}1.00 \\
-0.18\end{array}$ & 1 & 387 & $\begin{array}{l}386 \\
99.7 \%\end{array}$ & $\frac{1}{0.3 \%}$ \\
\hline & $\begin{array}{l}\text { Dls. - Koeffiz. } \\
\text { Gruppendurch- }\end{array}$ & 2 & 149 & 14 & $\begin{array}{l}135 \\
90.6 \%\end{array}$ \\
\hline & D & & \multirow{2}{*}{\multicolumn{3}{|c|}{$\begin{array}{l}\text { Korrekt klaseifizierte Fallo } \\
\text { in Prozenten: } 97.2\end{array}$}} \\
\hline $\begin{array}{l}\text { Gruppe } 1 \\
\text { Gruppe } 2\end{array}$ & $\begin{array}{r}1.59 \\
-4.13\end{array}$ & & & & \\
\hline
\end{tabular}

Signifikanz: * $p<0.001 \quad G=$ Gruppe

D. $F, \quad 2$

Wilks'Lambda 0.13

N

535 


\section{Literatur}

Akerlof, G. A.; Yellen, Y.: Efficiency Wage Models of the Labor Marke1, Cambridge University Press, Cambridge 1986

Albeda, W.: Arbeidsmarkt en Arbeidsorganisaties, in: Muysken, J.; Schreuder, H.: Economische Weienschappen: Eenheid in Verscheidenheid, Van Gorcum, Assen/Maastricht 1985, 133-137

Albert, $\mathrm{H}_{\text {:: }}$ Individuelles Handeln und soziale Steuerung, Die ökonomische Tradition und ihr Erkenntnisprogramm, in: Lenk, H. (Hsg.), Handlungstheorien interdisziplinär, Bd. 4, München 1977, $177 \mathrm{ff}$.

Albert, H.: Theorien in den Sozialwissenschaften, in: Albert, H. (Hsg.) Theorie und Realität, 2 Aufl, Tubingen 1972

Alchian, A.; Demsetz, H.: Production, Information Costs and Economic Organization, in: American Economic Review, 62, 1972, 777-795

Alexander, A. J.: Income, Experience, and the Structure of Intemal Labor Markets, in: Quarterly Journal of Economics. 1. 1974, 63-85

Algemeen jaarverslag, 1987 (Allgemeiner Tătigkeitsbericht des Krankenhauses 1987)

Althauser, R. P.: Kalleberg. A. L.: Identifying Career Lines and Intemal Labor Markets within Firms: A Study in the Interrelationships of Theory and Methods, in: Breiger, R. L. (ed.): Social Mobility and Social Structure, Cambridge University Press, Cambridge 1990, 308-356

Althauser, R. P.; Kalleberg, A. L.: Firms, Occupations and the Structure of Labor Markets, A Conceptional Analysis, in: Berg, I. (ed.), Sociological Perspectives on Labor Markets, Academic Press, New York $1981,119-145$

Anderson, J. C.; Milkovich, G. T.; Tsui, A.: A Model of Intra-Organizational Mobility, in: Academy of Management Review, 6, 1981, 529-538

Aoki, M. A.; Gustafsson, B.; Williamson O. E. (eds.): The Firm as a Nexus of Treaties, Sage Publications, London 1990

Arendt, H.: Vita Activa oder vom tätigen Leben, Piper Verlag, München 1981

Argyris, C.; Schön, D. A.: Organizational Learning, A Theory of Action Perspective, Addison-Wesley, London 1978

Arrow, K.: The Theory of Discrimination in Labor Markets, Princeton University Press, Princeton 1973 Atkinson, J.: Manpower Strategies for Flexible Organizations, in: Personnel Management, 16, 1984, 28-31 Alteslander, P.: Methoden der empirischen Sozialforschung, 5. Afl., Berlin 1984

Averitt, R. S.: The Dual Economy, New York 1968

Baaijens, J. M. J.; Have K. ten: Josepa, A. M.: Organisaties, Transacties en Strategieën, IVA, Tilburg 1989

Backhaus. J.; Nutzinger, H. G. (Hsg.):, Eigentumsrechte und Partizipation, Frankfurt 1982

Backhaus, J.: Arbeitsverhälmis und Beschäftigung, Frankfurt 1980

Balzer, A.: Firmeninte'rne Arbeitsmärkte, Ein Erklärungsheitrag aus Sicht der neuen institutionellen Ökonomie, Lang, Frankfurt 1987

Barbash, J.: The Future of Industrial Relations as an Academic Field, Konferenzpapier anläBlich der Konferenz: The Future of Industrial Relations in Europe, Maastricht 1990

Bamey, J. B.; Ouchi, W. G. (ed.).: Organizational Economics, Towards a New Paradigm for Understanding and Studying Organizations, Jossey-Bass, San Francisco 1986

Baron, J. N.; Bielby, W. T.: The Organization of Work in Segmented Economy, in: American Sociological Revie'w, 49, 1984, 454-473

Baron, J. N.; Bielby W. T.: Bringing the Firms Back in: Stratification, Segmentation, and the Organization of Work, in: American Sociological Review, 45, 1980, 737-765 
Baron, J. N.; Davis-Blake, A.; Bielby, W. T.: The Structure of Opportunity: How Promotion Ladders Vary within and among Organizations, in: Administrative Science Quarterly, 31, 1986, 248-273

Bechtle, G.: Betrieb als Strategie; Theoretische Vorarbeiten zu einem industriesoziologischen Konzept. Campus, Frankfurt 1980

Becker, H.: Eine transaktionskostentheoretische Interpretation interner Arbeitsmärkte, Duncker/Humblot, Berlin 1985

Behrens, H.: Die Reservearmee im Betrieb - Machtheoretische Überlegungen zu den Konzepten der "Kontrolle", der "Eigentumsrechte" und der "Sozialen SchlieBung, in: Jürgens, U.; Naschold, F. (Hsg.), Arbeitspolitik: Materialien zum Zusammenhang von politischer Macht, Kontrolle und betrieblicher Organisation der Arbeit, Opladen 1984, 133-154

Bellmann, L.: Senioritätsentlohnung, betriebliche Hierarchie und Arbeitsleistung: Eine theoretische und empirische Untersuchung zur Lohnstruktur, Campus, Frankfurt 1986

Benveniste, G.: Professionalizing the Organisation; Reducing Bureaucracy to Enhance Effectiveness, Jossey-Bass, London 1987

Berg, I. (ed.): Sociological Perspectives on Labor Markets, Academic Press, London 1981

Bergeijk, C. van, Grip, de A.: Bestaan en ontwikkelingen van inteme Arbeidsmarkten in Nederland, in: Sociaal Maandblad Arbeid, Juni, 1986, 437-451

Berger, J.: Offe, C.: Die Zukunft des Arbeitsmarktes: Primäres und sekundäres Machtgefälle, in: Offe, C.: Arbeitsgesellschaft, Strukturprobleme und Zukunftperspectiven, Campus. Frankfur 1984, 87-117

Berthel, J.: Karriere und Karrieremuster von Führungskräften, in: Kieser, A.; Reber, G.; Wunderer, R. (Hsg.), Handwörterbuch der Führung. Stuttgart 1987, $1183-1195$

Biehler, H.; Brandes, W.: Arbeissmarktsegmentation in der Bundesrepublik Deutschland. Campus. Frankfurt 1981

Biehler, H.; Brandes, W.; Buttler, F.; Gerlach, K.; Liepmann, P.: Arbeitsmarktstrukturen und Prozesse, Campus, Tübingen 1981

Biller, M.: Arbeitsmarkısegmentation und Ausländerbeschäftigung. Ein Beitrag zur Soziologie des Arbeitsmarktes mit einer Fallstudie aus der Automobilindustrie, Campus, Frankfurt 1989

Bills, D. B.: Costs, Commitment and Rewards: Factors Influencing the Design and Implementation of Intemal Labor Markets, in: Administrative Science Quarterly, 32, 1987, 202-221

Blau, P. M.; Ducan, O. D.: The American Occupational Structure, New York 1967

Blossfeld, H. P.: Berufseintitt und Berufsverlauf, in: Mitteilungen aus der Arbeitsmarkt und Berufsforschung, 18, 1985, 177-197

Blossfeld, H. P.; Hamerle, A.; Mayer, K. U.: Ereignisanalyse, Statistische Theorie und Anwendung in den Wirtschafits- und Sozialwissenschaften, Frankfur 1986

Böhle, F.; Altmann, N.: Industrielle Arbeit und soziale Sicherheit, Eine Studie über Risiken im ArbeitsprozeB und auf dem Arbeitsmarkt, München 1972

Bosssmann, E.: Untemehmung, Märkte, Transaktionskosten, in: Wirtschaftsstudium, 4, 1983, 105-111

Bolle, M. (Hsg.): Arbeitsmarktheorie und Arbeitsmarktpolitik, Leske, Opladen 1976

Bosch, G.: Hat das Normalarbeitsverhälnis eine Zukunft?, in: WSI Mitteilungen, 3, 1986, 163-176

Boulding, K.: The Impact of the Union, New York 1951

Boxman, E. A. W.: Contacten en carriere, een empirisch-theoretisch onderzoek naar de relatie tussen sociale netwerken en arbeidsmarktposities, Thesis Publishers, Amsterdam 1992

Brandes, W.; Buttler, F.: Die Unvermeidbarkeit interner Arbeitsmärkte, in: Reyher, L.; Kuhl, J. (Hsg.), Resonanzen. Arbeitsmarks- und Berufsforschung und Politik, Festschrift für Dieter Mentens; Beiträge zur Arbeitsmarkt- und Benufforschung BeitrAB III, Nümberg 1989

Brandes, W.; Liepmann, P.; Weise, P.: Untemehmungsverhalten und Arbeitsmarkt, in: Mehrwert, 22, 6-45 Brandes, W.; Weise, P.: Grundzilge einer Theorie institutionalisierter Arbeitsbeziehungen, in: Burtler, F.; Gerlach, K.; Schmiede R. (Hsg.): Arbeitsmarks und Beschäftigung, Neuere Beiträge zur institutionalistischen Arbeitsmarktanalyse, Campus, Frankfurt 1987, 64-94

Braverman, H.: Die Arheit im modernen Produktionsprozeß, Frankfurt 1977

Breiger, R. L. (ed.): Social Mobility and Social Structure, Cambridge University Press, Cambridge 1990. Brittain, J. W.; Wholey, D. R.: Structure as an Envisonmental Property: Industry Demographics and Labor Market Practices, in: Breiger, R. L. (ed.): Social Mobility and Sacial Structure, Cambridge University Press, Cambridge 1990, 155-182 
Brödner, P.: Fabrik 2000, Sigma, Berlin 1985

Brüderl, J.: Mobilitätsprozesse in Betrieben, Dynamische Modelle und empirische Befunde, Campus, Frankfurt 1991

Briderl, J.: Industries, Labor Markets, Firms and Occupational Careers: On which Level Does Structure Matter?, in: Mayer, K. U.; Tuma, N. B. (eds.), Applications of Event History Analysis in Life Course Research, Materialien aus der Bildungsforschung, 30, Berlin 1987, 140-161

Brüderl, J.: Diekmann, A.: Preisendörfer, P.: Verlaufsmuster innerbetrieblicher Aufstiegsmobilität: Turniermodelle, Pfadabhängigkeiten und "Frühstarteffekte", in: Köhler, $C_{\text {; }}$ Preisendörfer P. (Hsg.): Betrieblicher Arbeitsmarkt im Umbruch, Analysen zur Mobilitäl, Segmentation und Dynamik in einem Großbetrieb. Campus. Frankfurt 1989, 117-145

Buitendam, A.: Decentralization and the Governance of Employment Relationships: Human Resource Management between Labour Market and Organization, Research Memorandum, No. 4/3, Institute of Economic Research. Faculty of Economics, University of Groningen, Groningen 1991

Buitendam, A.: Arheidsmarkt, arbeidsorganisatie, arbeidsverhoudingen, Vraagstukken voor ondememing, vakbeweging en overheid, Kluwer, Deventer 1987

Burg. van der, B. 1.: Loophaanverschillen fussen mannen en vrouwen binnen arbeidsorganisaties, WoltersNoordhoff, Groningen 1992

Burns. T.; Stalker, G. M.: The Management of Innovation, London 1961

Butler, John E.; Ferris, Gerald F.; Napier, Nancy, K.; Strategy and Human Resources Management, South-Western Publishing Co., Cincinnati/Ohio 1991

Butter, F.; Gerlach K.; Liepmann, P.: Messung und Interpretation betriebsinterner Arbeitsmarktbewegungen - ein empirischer Beitrag zur nicht-marktgesteuerten Allokation von Arbeitskcäften, in: Sengenberger, W. (Hsg.), Der gespaltene Arbeitsmarkt, Frankfurt 1978, 185-224

Buttler, F.; Gerlach, K.; Schmiede R. (Hsg.): Arbeitsmarkı und Beschäftigung, Neuere Beiträge zur institutionalistischen Arbeitsmarktanalyse, Campus, Frankfur 1987

Cain, G. C.: The Challenge of Segmented Labor Market Theories to Orthodox Theory: A Survey, in: Journal of Economic Literature. 14, 1976, 1215-1257

Cappelli, P; Cascio, W. F.: Why some Jobs Command Wage Premiums: A Test of Career Toumament and Internal Labor Market Hypotheses, in: Academy of Management Journal, 34, 1991, 848-868

Carmichael, H. L: Efficiency Wage Models of Unemployment, One View, in: Economic Inquiry, 28, 1990, 269-295

Carroll, G. R.; Mayer, K. U.: Organizational Effects in the Wage Attainment Process, in: Arbeitspapier, Sonderforschungsbereich 3, 95. Frankfurt/Mannheim 1983

Chandler, A. D. Jr.: Strategy and Structure, Chapters in the History of the American Industrial Enterprise. the MIP Press, Cambridge, Massachusetts 1962

Clegg. S.: Modern Organization, Sage, London 1990,

Coase. R. H.: The Nature of the Firm, in: Economica, 4, 1937, 386-405

Cohen, Y.: Pfeffer, J.: Organizational Hiring Standards, in: Administrative Science Quarterly, 31, 1986, $1-24$

Cole, R. E.: Work, Mobility, and Participation. A Comparative Study of American and Japanese Industry, University of California Press, Berkeley/London 1979

Commons, J. R.: Institutional Economics, Its Place in Political Economy, Macmillan, New York 1934

Creedy, J.; Whitfield, K.: The Economic Analysis of Internal Labour Markets, in: Bulletin of Economic Research, 40, 1988, 247-269

Crozier, M.: The Bureaucratic Phenomenon, The University of Chicago Press, Chicago 1964

Deeke A.; Fischer J.: Wege der Rekrutierung auf dem Arbeitsmarkt - Ausgewählte erste Ergebnisse einer schriftlichen Betriebsbefragung, Arbeitspapier 1986-6, Arbeitskreis sozialwissenschaftliche Arbeitsmarkforschung (SAMF), 1986

Demsetz, H.: Efficiency, Competition, and Policy, the Organization of Economic Activity, Volume II, Basil Blackwell, Oxford, New York 1989

Deutschmann, C.: Der "Betriebsclan". Der Japanische Organisationstypus als Herausforderung an die soziologische Modemisierungtheorie, in: Soziale Welt, 38, 1987, 133-147 
Diekmann, A.; Preisendörfer, P.: Fluktuation und Beschäftigungsstabilität in einem bundesdeutschen GroBbetrieb, in: Köhler, C.; Preisendörfer P. (Hsg.): Betrieblicher Arbeitsmarks im Umbruch, Analysen zur Mobilität, Segmentation und Dynamik in einem Großbetrieb. Campus, Frankfurt 1989, 47-71

Dillard, J. F.; Ferris, K. R.: Individual Behavior in Professional Accounting Firms: A Review and Synthesis, in: Journal of Accounting Literature, 8, 1989, 208-234

Diprete, T. A.: The Bureaucratic Labor Market: The Case of the Federal Civil Service, Plenum Press, New York 1989

DiPrete, T. A.: Horizontal and Vertical Mobility in Organizations, in: Administrative Science Quarterly, 32, 1987a, 422-444

DiPrete, T. A.: The Professionalization of Administration and Equal Employment Opportunity in the U.S. Federal Government, in: American Journal of Sociology, 93. 1987b, 119-140

DiPrete, T. A.; Soule, W. T.: Gender and Promotion in Segmented Job Ladder Systems, in: American Sociological Review, 53, 1988, 25-40

DiTomaso, N.: Income Determination in Three Intemal Labor Markets, in: Farkas, G.; England, P. (eds.), Industries, Firms, and Jobs. Sociological and Economic Approaches, Plenum Press, New York/London 1988, 217-246

Doeringer, P. B.: Determinants of the Structure of Industrial Type Intemal Labor Markets, in: Industrial Labor Relations Review, 20, 1967, 206-220

Doeringer, P. B.; Piore, M. J.: Internal Labor Markets and Manpower Analysis, Lexington 1971

Doeringer, P. B.; Piore, M. J.: Intemal Labor Markets and Manpower Analysis: A Second Look, in: Doeringer, P. B.; Piore, M. J.; Internal Labor Markets and Manpower Analysis, Reprint, London 1985, Sharpe, Ix-xxxv; Mit Ausnahme des 'second look' identisch mit der Ausgabe von 1971

Domsch, M.; Schneble, A.: Personalinformation für Führungskräfte, in: Kieser, A.; Reber, G.; Wunderer, R. (Hsg.), Handwörterbuch der Führung, Stuttgart 1987, 1668-1680

Douma, S.; Schreuder, H.: Economic Approaches 10 Organizations, Prentice Hall, London 1991

Dow, G. K.: The Function of Authority in Transaction Cost Economics, in: Journal of Economic Bchavior and Organization, 8. 1987, 13-38

Drumm, H. J.: Personalwirtschafislehre, Springer Verlag, Berlin Heidelberg 1989a

Drumm, H. J.: Unternehmerische Arbeitsmarkfforschung - strategische Begründung, konzeptionelle Möglichkeiten und Grenzen, in: Mitteilungen aus der Arbeitsmarks- und Berufsforschung, 22, 1989b, 348-354

Drugger, W. M.: The Transaction Cost Analysis of Oliver Williamson: A New Synthesis?, in: Journal of Economic Issues, XVIl, 1983, 95-114

Drugger, W. M.: The Administered Labor Market: An Institutional Analysis, in: Journal of Economic Issues, 15, 1981, 387-407

Dunlop, J. T.: Industrial Relations Systems, New York 1958

Dunlop, J. T.: The Task of Contemporary Wage Theory, in: Taylor, G. W.; Pierson, F. C. (eds.), New Concepts in Wage Determination, McGraw Hill, New York 1957, 3-27

Edwards, R.: Contested Terrain: The Transformation of the Workplace in the Twentieth Century, Basic Books, New York 1979

Edwards, R. C.; Reich, M.; Gordon, D. M.: Labor Market Segmentation, Lexington 1975

Elbaum, B.: The Intemalization of Labor Markets: Causes and Consequences, in: American Economic Review, 73, 1983, 260-265

Elsik, W.: Strategisches Personalmanagement, Konzeptionen und Konsequenzen, Hampp Verlag, München 1992

Endruweit, G.: Social Boundaries of Labour Markets, in: Dlugos, G.; Dorow, W.; Weiermair, K. (eds.); Management under Differing Lahour Market and Employment Systems, de Gruyter, Berlin 1988

Fehr, E.: (Un)freiwillige Arbeitslosigkeit durch Effizienzlöhne? Eine Gegenkritik, in: Gerlach, K.; Hübler, O. (Hsg.): Effizienzlohntheorie, Individualeinkommen und Arbeitsplatzwechsel, Campus, Frankfurt 1989

Feijen, M. H. P. F.: Boundary Management: A Dynamic Balance Between Rules and Space, in: Human Systems Management. 9. (1990), 257-265

Fombrun, C. J.; Corporate Culture and Competitive Stategy, in: Fombrun, C. J. et al., Strategic Human Resources Maragement, New York 1984, 203-216 
Francis, A.: Markets and Hierarchies: Efficiency or Domination?, in: Francis, A.; Turk, J.; Willman, P. (eds.): Power, Efficiency and Institutions, A Critical Appraisal of the Markets and Hierarchies Paradigm, Heinemann Educational Books, London 1983, 105-116

Francis, A.; Turk, J.; Willman, P. (eds.): Power, Efficiency and Institutions, a Critical Appraisal of the Markets and Hierarchies Paradigm, Heinemann Educational Books, London 1983

Freiburghaus, D.: Arbeitsmarktsegmentation; Wissenschaftliche Modeerscheinung oder arbeitsmarkttheoretische Revolution?, in: Beifräge aus der Arbeitsmarks- und Berufsforschung, 33, 1979, $159 \mathrm{ff}$.

Freiburghaus, D.; Schmid, G.: Theorie der Segmentienung von Arbeitsmärkten, in: Leviathan, 3, 1975, 417 ff.

Fricke, W.: Arbeitsorganisation und Qualifikation, Ein industriesoziologischer Beitrag zur Humanisienung der Arbeit, Bonn 1975

Gabriel, J.: Flexibilisierung der Arbeit und wirtschafiliche Instabilität, Überlegungen zur Theorie und Empirie des Beschäftigungsverhaltens von Unternehmen bei Unsicherheit. Minerva Publikation, München 1985

Gaertner, K. N.: The Structure of Organizational Careers, in: Breiger, R. L. (ed.): Social Mobility and Social Structure. Cambridge University Press, Cambridge 1990, 133-154

Gerlach, K.; Hübler O.: Einführung, in: Gerlach, K.; Hübler O. (Hsg.): Betriebszugehörigkeitsdauer und Mobilität - Theorerische und empirische Analysen, Arbeitspapier 1990 - 4, SAMF, 2-7

Gerlach, K.; Hübler, O.: Effizienzlöhne und individuelles Einkommen - Einige einführende Aspekte, in: Gerlach, K.; Hübler, O. (Hsg.): Effizienzlohntheorie, Individualeinkommen und Arbeitsplarzwechsel, Campus, Frankfurt 1989

Gerpott. T. J.: Karrierecntwicklung von Industrieforschern, Positionswechsel in derselben Untemehmung? de Gruyter, Berlín 1988

Gijsbers, J.; Sprenger, J.: De dreiging van de ongeorganiseerde arbeidsmarkt; Gesprek met W. Albeda, in: Zeggenschap. Tijdschrift voor vakbewegingsvraagstukken, 3, 1990, 11-14

Gijsel, P. De, Muysken J.; Wolfs, G. L. M.: Interne Arbeidsmarkten en Beloning bij Mannen en Vrouwen in Nederland, in: Mens en Maatschappij, 3, 1991, 257-276

Glebbeek, A. C.: Arbeidsmarkt, Arbeitsorganisatie en Loopbanen; Het Structuralistische Programma in het Mobiliteitsonderzoek, in: Buitendam (Red.) Arbeidsmark, Arbeidsorganisatie, Arbeidsverhoudingen; Vraagstukken voor Onderneming, Vakbew'eging en Overheid, Deventer 1987, $32-54$

Goldner, F.H.: Ritu, R. R.: Professionalization as Career Immobility, in: American Journal of Sociology, 13. 1967, 489-502

Gorz, A.: Kritik der ökonomischen Vernunfi, Rotbuch Verlag, Berlin 1989

Gouldner, A. W.: Patterns of Industrial Bureaucracy, New York 1964

Grandjean, B.: History and Career in a Bureaucratic Labor Market, in: American Journal of Sociology, 86. 1981, 1057-1092

Granovetter, M.: The Sociological and Economic Approaches to Labor Market Analysis: A Social Structural View, in: Farkas, G.; England, P. (eds.), Indusmies, Firms, and Jobs; Sociological and Economic Approaches, Plenum Press, New York/London 1988, 187-217

Granovetter, M.: Small is Beautiful: Labor Markets and Establishment Size, in: American Sociological Review. 49, 1984, 323-334

Greve, A.: The Social Structure and Processes of Career Mobility; An Empirical Study on Internal Labor Markets in Norway, Working Paper, Wissenschaftszentrum Berlin, Berlin 1989

Grip, A. De: Inteme Arbeidsmarkttheorieën: Een Overzicht, in: Maandschriff Economie, 49, 1985, 333-345

Grochla, E., Grundlagen der organisatorischen Gestaltung, Stuttgart 1982

Grochla. E.: Organisationstheorie, in: Handwörterbuch der Organisation, 2 Aufl., Snuttgart 1980, 1795-1814

Groenewegen, J.: Planning in een markteconomie, Indicatieve planning, industriebeleid en de rol van de publieke onderneming in Frankrijk in de periode 1981-1986, Delft 1989

Grüner, H.: Karrieremuster und soziale Differenzienung im betrieblichen Arbeitsmarkt, in: Kobler, C.: Preisendörfer P. (Hsg.): Betrieblicher Arbeitsmarks im Umbruch, Analysen zur Mobilität, Segmentation und Dynamik in einem Großbetrieh. Campus Verlag, Frankfur 1989, 93-116

Guggenberger, B.: Wenn uns die Arbeit ausgeht, Die aktuelle Diskussion um Arbeitszeitverkürzung, Einkommen und die Grenzen des Sozialstaats, Hanser Verlag, München 1988 
Gunz, H.: Careers and Corporate Cultures, Managerial Mobility in Large Corporations. Basil Blackwell, Oxford 1989

Gunz, H.: The Dual Meaning of Managerial Careers: Organizational and Individual Levels of Analysis, in: Journal of Management Studies, 26, 1989, 225-250

Gunz, H.: Organizational Logics of Managerial Careers, in: Organization Studies, 9, 1988, 529-554

Gütermann, C.: Zur Konzeption einer hetrieblichten Arbeitsmarktstrategie, Strategie- und Informationsmanagement, Hampp Verlag, München 1989

Gutteridge, T. G.: Organizational Career Development Systems: The State of the Practice, in: Hall, D. T. et al. (eds.): Career Development in Organizations, San Francisco 1986

Hachen, D. S.: Three Models of Job Mobility in Labor Markets, in: Work and Occupations, 17, 1990. 320-354

Hage, J; Aiken, M.: Routine Technology, Social Structure, and Organizational Goals, in: Administrative Science Quarterly, 14, 1969, 366-376

Halaby, C. N.: Bureaucratic Promotion Criteria, in: Adminstrative Science Quarterly, 23. 1978, 466-484

Hall, D. T.: Career Development in Organizations, Jossey-Bass, San Francisco 1986

Hal, D. T.: Career Development in Organizations: Where do we go from here?, in: Hall, D. T. et al. (eds.): Career Development in Organizations, San Francisco 1986

Hall, D. T.: Introduction: An Overview of Current Career Development, Theory, Research, and Practice, in: Hall, D. T. et al. (eds.): Career Development in Organizations, San Francisco 1986

Ham, J. C. van, Paauwe, J.; Williams, A. R. T.: Human Resources Management en Transactiekostenbenadering, in: Economisch Statistische Berichten, 23-11, 1988, 1109-1112

Ham, J. C. van; Paauwe, J.; Williams, A. R. T.; Flexibiliteit en stabiliteit vanuit individu en organisatic, in: Buitendam, A. (Hsg.) Arbeidsmarkt, Arbeidsorganisatie, Arbeidsverhoudingen, Vraagstukken voor onderneming, vakbeweging en overheid; Kluwer: Deventer 1987

Hamermesh, D. S.; Rees, A.: The Economics of Work and Pay, 4 ed., New York 1988

Have, ten, K.: Vissers, A.: Arbeid tussen marks en organisatie, een verhandeling over kwaliteit van arbeid in relatie tot arbeidsmarktgedrag, OSA-werkdocument, Tilburg 1987

Heinen, E.: Unternehmenskultur, Perspektiven für Wissenschaft und Praxis, Oldenbourg Verlag, München 1987

Hendrikse, G. W. J.; Schreuder, H.: Economische Organisatietheorieèn, in: Economisch Statistische Berichten, 72, 1987, 810-815

Hendry, C.; Pettigrew, A.: Human Resource Management: An agenda for the 1990s, in: International Journal of Human Resource Management, 1, 1990, 17-43

Hodgson, G. M.: Economics and Institutions, A Manifesto for a Modern Institutional Economics, Polity Press, Worcester 1988

Hofstede, G.: Cultures and Organizations, Software of the Mind, Intercultural Cooperation and Its Importance for Survival; McGraw-Hill, London 1991

Hofstede, G.: Culture's Consequences, Sage, Beverly Hills 1980

Hondeghem, A.: De loopbaan van de amblenaar. Tussen droom en werkelijkheid, Leuven 1990

Hoof, J. J. van: Balanceren tussen prestaties en tegenprestaties, in: Tijdschrift voor Arbeidsvraagstukken, 7, 1991/3, 56-63

Hoof, J. J. van: De Arbeidmarks als Arena, Arbeidsmarktproblemen in Sociologisch Perspectief. SUA. Amsterdam 1987

Iterson, A. van: "Vader, Raadgever en Beschermer", Petrus Regout en zijn arbeiders 1834-1870; Maastricht 1992

Iterson, A. van: Beheersing van Arbeid, Problemen van werving, behoud en beheersing van arbeid tijdens de opkomst van het fabriekssysteem, Research Memorandum, Rijksuniversiteit Limburg. Maastricht 1989

Jahoda, M.: Wieviel Arbeit braucht der Mensch?, Arbeit und Arbeitslosigkeit im 20 Jahrhundert, Beltz Verlag, Weinheim 1983

Joehr, W. A.: Zur Arbeitslosigkeit der Gegenwart. Wie ist langandauernde allgemeine Massenarbeitslosigkeit bei vorwiegend markswirtschaflicher Ordnung möglich?, Tübingen 1986

Johnson, G.: Strategic Change and the Management Process, Basil Blackwell, Oxford 1987

Jürgens U.; Naschold, F. (Hsg.): Arbeitspolitik, Opladen 1984 
Kalleberg, A. L.: Linking Macro and Micro Levels: Bringing the Workers back into the Sociology of Work, in: Social Forces, 67, 1989, 582-593

Kalleberg, A. L.: Comparative Perspectives on Work Structures and Inequality, in: Annual Review Sociology, 14, 1988, 203-225

Kalleberg, A. L.; Berg, I.: Work and Industry, Structures, Markets, and Processes, Plenum Press, London 1987

Kalleberg, A. L.; Wallace M.; Althauser R. P.: Economic Segmentation, Worker Power, and Income Inequality, in: American Journal of Sociology, 1981, 651-683

Kanter, R. M.: Variations in Managerial Career Structures in High Technology Firms, in: Osterman, P. (ed.), Internal Labor Markets, the MIT Press, Cambridge 1984, 109-131

Kanter, R. M.: Men and Women of the Corporation, Basic Books, New York 1977

Karpik, L.: Les politiques et les logiques d'action de la grande enterprise industrielle, in: Sociologie du Travail, 1972, 82-115

Keizer, P. K.; Soeters, J.: Economie. Sociologie en Psychologie, Visies op Integratie, Van Gorcum, Assen/Maastricht 1987

Keller, B.: Noch mehr De-Regulierung - oder stärkere Re-Regulierung?, in: WSI Mitteilungen, 43, 1990, 366-376

Kerckhoffs, C.; Wolfs, G.: Duration of Job Tenure in the Dutch Economy, Research Memorandum! Rijksuniversiteit Limburg, Maastricht 1991

Kem, H.; Schumann, M.: Das Ende der Arbeitsteilung, Rationalisierung in der industriellen Ptoduktion, Beck, München 1984

Kerr, C.: The Balkanization of Labor Markets, in: Bakke, E. W. et al. (eds.), Labor Mobility and Wage Determination, New York 1954, 92 ff.

Kieser, A.; Kubicek, H.: Organisationstheorien I+ II, Stuttgart 1978

Kieser, A.; Reber G.; Wunderer R.: Handwörterbuch der Führung, Stuttgart 1987

Kluyomans, F.: Ontwikkelingen in het personeelsmanagement: een historische schets, in: Kluytmans, F.: Hancke, C.: Personeelsmanagement. Kluwer, Heerlen 1990, 24-39

Kluytmans, F; Paauwe, J.: HRM denkbeelden: De balans opgemaakt, Een aanzet voor een onderzoeksagenda, in: Mens en organisatie, 4, 1991, 279-303

Köhler, C.; Preisendörfer P. (Hsg.): Betrieblicher Arbeitsmarkt im Umbruch, Analysen zur Mobilität, Segmentation und Dynamik in einem Großbetrieb, Campus, Frankfurt 1989

König. R.: Praktische Sozialforschung: Das Interview, in: König, R. (Hsg.) Praktische Sozialforschung. Bd. 1, Köln 1979, 13-33

Konrad. A. M.; Pfeffer J.: Do You Get What You Deserve? Factors affecting the Relationship between Productivity and Pay. in: Administrative Science Quarterly, 35, 1990, 258-285

Kubicek, H.; Welter, G.: Messung der Organisationsstruktur, Stuttgart 1985

Kunst, P.; Soeters, J.: Work Council Membership and Career Opportunities, in: Organization Studies, 12. $1991,75-93$

Kunst, P.; Schreuder, H.; Spangenberg. J.; Romme, S.: Structure and Control: An Empirical Exploration, Research Memorandum, University of Limburg, Maastricht 1989

Lammers, C. G.: Organisaties Vergelijkenderwijs. Spectrum, Utrecht 1983

1.angley. A.: In Search of Rationality: The Purposes Behind the Use of Formal Analysis in Organizations, in: Administrative Science Quarterly, 34, 1989, 598-631

Larm, T.: Arbeitsmarkttheorie und Arbeitslosigkeit, Systematik und Kritik arbeitsmarkttheoretischer Ansätze, Frankfurt 1982

Laske, S.: Personalentwicklung als Führungsmittel, in: Kjeser, A.; Reber, G.; Wunderer, R. (Hsg.), Handwörterbuch der Führung. Stuttgart 1987, 1647-1668

Lawrence, P. R.; Lorsch, J. W.: Organization and Environment, Homewood 1969

Lawrence, P. R.; Lorsch. J. W.: Developing Organizations; Diagnosis and Action, Addison-Wesley, 1969

Lee, G; Loveridge, R. (ed.): The Manufacture of Disadvantage Stigma and Social Closure, Open University Press, Milton Keynes 1987

Lincoln, J. R.; Kalleberg. A. L.: Culture, Control and Commitment, A Study of Work Organizations and Work Attitudes in the United States and Japan; Cambridge Uni 'ersity Press Cambridge 1990 
Lindbeck, A.; Snower, D. J.: The Insider-Outsider Theory of Employment and Unemployment, the MIT Press, London 1988

London, M.; Mone, E. M.: Career Management and Survival in the Workplace. Helping Employees Make Tough Career Decisions, Stay Motivated, and Reduce Career Stress, Jossey-Bass Publishers, London 1987

Lorenz, W.: Dauer der Betriebszugehörigkeit aus Sicht der Unternehmung, in: Gerlach, K.; Hübler O. (Hsg.): Betriebszugehörigkeilsdauer und Mobilität - Theoretische und empirische Analysen, Arbeitspapier 1990 - 4, SAMF, 33-60

Loveridge, R.: Introduction: Stigma: The Manufacture of Disadvantage, in: Lee, G; Loveridge, R. (ed.): The Manufacture of Disadvantage Stigma and Social Closure, Open University Press, Milton Keynes 1987, 2-17

Loveridge, R.: Social Accommodations and Technological Transformations: The Case of Gender, in: Lee, G; Loveridge, R. (ed.): The Manufacture of Disadvantage Stigma and Social Closure, Open University Press, Milton Keynes 1987, 176-197

Loveridge, R.: Sources of Diversity in Internal Labour Markets, in: Sociology, 17, 1983, 44-62

Loveridge, R.; Mok, A. L.: Labor Market Segmentation: A Critique, Nijhoff Social Sciences Division, Den Haag 1979

Loveridge, R.; Mok, A. L.: Labor Market Segmentation: A Critique, Nijhoff Social Sciences Division, Den Haag 1979

Luhmann, N.: Einfache Sozialsysteme, in: ders. Soziologische Aufklärung 2, Aufsärze zur Theorie der Gesellschaft, Opladen 1975

Lulofs, J. G.: Vakbeweging tussen arbeidsmarkt en arbeidsorganisatie, in: Buitendam, A.: Arbeidsmarkt, arbeidsorganisatie, arbeidsverhoudingen, Vraagstukken voor ondememing, vakbeweging en overheid, Kluwer, Deventer 1987, 153-178

Lutz, B.: Arbeitsmarktstruktur und hetriebliche Arbeitskräftestrategie, Eine theoretisch-historische Skizze zur Entstehung betriebszentrierter Arbeitsmarktsegmentation, Campus. München 1987

Lutz, B.; Sengenberger. W.: Arbeitsmarktstruktur und öffentliche Arbeitsmarktpolitik, eine kritische Analyse von Zielen und Instrumenten; Göttingen 1974

Maanen, J. Van: Experiencing Organization: Notes on the Meaning of Careers and Socialization, in: Maanen, J. Van (ed.), Organizational Careers: Some New Perspectives, London 1977, 15-48

Maanen, J. Van: Introduction: The Promise of Career Studies, in: Maanen, J. Van (ed.), Organizutional Careers: Some New Perspectives, London 1977, 1-12

Maanen, J. Van: Summary: Toward a Theory of the Career, in: Maanen, J. Van (ed.), Organizational Careers: Some New Perspectives, London 1977, 161-180

Mace, J.: Intemal Labour Market for Engineers in British Industry, in: British Journal of Industrial Relations, 17, 1979, 50-63

McConnell, C. R.; Brue, S. L.: Contemporary labor economics, 2ed., McGraw-Hill, New York 1989

Malcomson, J. M.: Work Incentives, Hierarchy and Internal Labor Markets, in: Journal of Political Economy, 92, 1984, 486-507

Mayer, K. U.: Carroll, G. R.: Jobs and Classes: Structural Constraints on Career Mobility, in: Mayer, K. U.: Tuma, N. B. (eds.), Applications of Event History Analysis in Life Course Research, Materialien aus der Bildungsforschung, 30, Berlin 1987, 87-139

Mayer, K. U.; Tuma, N. B.: Applications of Event History Analysis in Life Course Research. Materialien aus der Bildungsforschung, Berlin 1987

Mayntz, R.: Forschungsmanagement, Steuerungsversuche zwischen Scylla und Charybdis; Probleme der Organisation und Leitung von hochschulfreien offentlich finanzierten Forschungsinstituten, Westdeutscher Verlag, Opladen 1985

Mayntz, R. (Hsg.): Bürokratische Organisation, Köln 1968

Mayrhofer, W.: Trennung von der Organisation, Vom Outplacement zur Trennungsberatung, Deutscher Universitäts Verlag, Wiesbaden 1989

Michaelis, E.: Organisation unternehmerischer Aufgaben, Transaktionskosten als Beurteilungskriterium, Lang, Frankfurt 1985

Miles, R. E.; Snow, C. C.: Organizational Strategy, Structure and Processes, McGraw-Hill, New York 1978 
Miller, D.: Configurations of Strategy and Structure: Towards a Synthesis, in: Strategic Management Journal, 7, 1986, 233-249

Miner, A. S.: Idiosyncratic Jobs in Formalized Organizations, in: Administrative Science Quarterly, 32 , 1987, 327-351

Mintzberg, H.: The Design School: Reconsidering the Basic Premises of Strategic Management, in: Strategic Management Journal, 11, 1990, 171-195

Mintzberg. H.: Mintzberg on Management, Inside our Strange World of Organizations, the Free Press, New York 1989

Mintzberg, H.: Power in and Around Organizations, Prentice-Hall Inc., Englewood Cliffs, New Jersey 1983

Mintzberg, H.: The Structuring of Organizations, Prentice Hall Inc., Englewood Cliffs, New Jersey 1979

Mintzberg, H.; Waters, J. A.: Of Strategies, Deliberate and Emergent, in: Strategic Management Journal, $6,1985,257-272$

Miyazaki. H.: The Rat Race and Internal Labor Markets, in: Bell Journal of Economics, 8, 1977, $394 \mathrm{ff}$.

Morgan, G.: Images of Organization, Sage Publications, London 1986

Muysken, J.: Neubourg, C. de: Introduction, in: Muysken, J.; Neubourg, C. De: Unemployment in Europe, Macmillan Press, London 1989, 1-8

Neuberger, O.; Rieckmann, H.; Wächter, H.; Schröder, W.; Schartner, H.: Der Mensch ist Mittelpunkt, der Mensch ist Mittel. Punkt. Acht Thesen zum Personalwesen, in: Personalführung, 1, 1990, 3-10

Nicholson, N.: A Theory of Work Role Transitions, in: Administrative Science Quarterly, 29, 1984, 171191

Nonusis, M. J.: SPSS-User's Guide. Chicago 1986 und 1990

Norusis, M. J.: SPSSIPC Base Manual; Chicago 1988

Norusis, M. J.: SPSS/PC + Advanced Statistics V2.0, Chicago 1988

Ochsenbauer, C.: Organisatorische Alternativen zur Hierarchie, München 1989

Offe, C.: Arbeilsgesellschaft, Strukturprobleme und Zukunftperspektiven, Campus, Frankfurt 1984

Offe, C.: Arbeit als soziologische Schlüsselkategorie?, in: Offe, C.: Arbeitsgesellschaft, Strukturprobleme und Zukunftperspektiven, Campus, Frankfurt 1984, 13-43

Offe, C.; Hinrichs, K.: Sozialökonomie des Arbeitsmarktes und die Lage benachteiligter Gruppen von Arbeitnehmem, in: Offe, C. (Hsg.), Opfer des Arbeilsmarktes, Zur Theorie der strukturierten Arbeitslosigkeit, Neuwied 1977

Okun, A.: Prices and Quantities: A Macroeconomic Analysis, Brookings Institute, Washington 1981

Osterman, P.: Employment Futures, Reorganization, Dislocation, and Public Policy, Oxford University Press, Oxford 1988

Osterman. P.: Choice of Employment Systems in Internal Labor Markets, in: Industrial Relations, 26, $1987,46-67$

Osterman, P. (eds.): Internal Labor Markets, Cambridge 1984

Osterman, P.: Employment Structures within Firms, in: British Journal of Industrial Relations, 20, 1982, 349-361

Ouchi, W. G.: Markets, Bureaucracies and Clans, in: Administrative Science Quarterly, 25, 1980, 129-141

Ouchi, W. G.; Jaeger, A. M.: Type Z Organization: Stability in the Midst of Mobility, in: Academy of Management Review, April 1978, 305-314

Ours, J. C. Van, Zoethout, T.: De Interne Arbeidsmarkt van de Gemeente Amsterdam, Research Memorandum, VU, Amsterdam 1990

Paauwe, J.: Sociaal Ondernemingsbeleid: Tussen Dwang en Ambities, Een theoretisch-empirisch onderzoek naar de vormgeving van het sociaal beleid van ondernemingen ten tijde van recessie en herstel, Samson, Alphen Aan de Rijn, 1989

Patzelt, W. J.: Einfuihrung in die soziahwissenschaftliche Statistik, Oldenbourg, München 1985

Pennings, J. M. et al.: Orgunizational Strategy and Change, Jossey Bass, San Francisco 1985

Perrow, C.: Complex Organizations, A Critical Essay, New York 1986

Peters, T. J.; Waterman, R. H.: In Search of Excellence, Lessons from America's Best-Run Companies, Harper \& Row, New York 1982 
Pfeffer, J.: Organizational Demography: Implications for Management, in: California Management Review, $28,1985,67-81$

Pfeffer, J.: Organizations and Organization Theory, New York 1982

Pfeffer, J.; Cohen, Y.: Determinants of Intermal Labor Markets in Organizations, in: Administrative Science Quarterly, 29, 1984. 550-572

Pfriem, H.: Konkurrierende Arbeitsmarkstheorien. Neoklassische, duale und radikale Ansätze, Frankfurt 1979

Picot, A.: Transaktionskostenansatz in der Organisationstheorie: Stand der Diskussion und Aussagewert, in: Die Betriebswirtschafi, Zeitschrift für Handelswissenschaft und Handelspraxis, 42, 1982, 267-284

Piore, M. J.: Lemprozesse, Mobilitälsketten und Arbeitsmarktsegmente, in: Sengenberger, W. (Hsg.), Der gespaltene Arbeitsmarkt, Frankfurt 1978, 67-98

Piore, M. J.: Notes for a Theory of Labor Market Stratification, in: Edwards, R. C.; Reich, M.; Gordon, D. M. (eds.); Labor Market Segmentation, Lexington, Mass. 1975, 125-150

Piore, M. J.: Fragments of a "Sociological" Theory of Wages, in: IRRA 25th Anniversary Proceedings $1972,286-305$

Piore, M. J.: Sabel, C. F.: The Second Industrial Divide, Basic Books, New York 1984; deutsche Übersetzung: Das Ende der Massenproduktion: Studie über die Requalifizierung der Arbeit und die Rückkehr der Ökonomie in die Gesellschaft, Berlin 1985

Popper, K. R.: Naturgesetze und Theoretische Systeme, in: AJber, H. (Hsg.), Theorie und Realität 2. Afl., Tübingen 1972

Preisendörfer, P.: Organisationsdynamik und Karrieremuster, in: Köhler, C.; Preisendörfer P. (Hsg.): Betrieblicher Arbeitsmarks im Umbruch, Analysen zur Mobilität, Segmentation und Dynamik in einem Großbetrieb, Campus, Frankfun 1989, 233-256

Preisendörfer, P.: Organisationsökologie: Eine neue Perspektive zur Untersuchung des Wandels von Organisationsstrukturen, in: Österreichischen Gesellschafi für Soziologie, 13, 1988, 24-29

Preisendörfer, P.: Organisationale Determinanten beruflicher Karrieremuster, Theorieansäzze, methodische Zugangswege und empirische Befunde, in: Soziale Welt, 38, 1987, 211-226

Preisendörfer, P.; Wallaschek, M.: Methodische Probleme der Analyse von Betriebszugehörigkeitsdauem, in: Betrieblicher Arbeitsmarks im Umbruch, Analysen zur Mobilität, Segmentation und Dynamik in einem Großbetrieb, Campus, Frankfurt 1989, 33-45

Pugh, D. S.; Hickson, D. J.: Organizational Structure in its Context, The Aston Programme I; Westmead und Lexington 1976

Putterman, L: Corporate Govemance, Risk-Bearing and Economic Power: A Comment on Recent Work by Oliver Williamson, in: Journal of Institutional and Theoretical Economics, 148, 1987, 422-434

Ramstad, Y.: Institutional Economics: How Prevalent in the Labor Literature?, in: Journal of Economic Issues, 15, 1981, 339-350

Reve, T.: The Firm as a Nexus of Internal and External Contracts, in: Aoki, M. et al. (eds.), The Firm as a Nexus of Threaties, Sage, London 1990, |33-16|

Rieckmann, H.: Organisationsentwicklung als Element strategischer Personalpolitik und Personalentwicklung, in: Personalführung, 7, 1989, 686-693

Romme, A. G. L.: A Self-Organization Perspective on Strategy Formation, Maastricht 1992

Romme, G.; Kunst, P.; Schreuder, H.; Spangenberg, J.: Assessing The Process and Content of Strategy in Different Organizations, in: Scandinavian Journal of Management, 6, 1990, 45-61

Romme, S.; Kunst P.; Schreuder H.; Spangenberg J.: Characteristics of Strategy in Different Organizational Structures: An Empirical Assessment, Working Paper, ELASM, Brussels 1989

Rosenbaum, J. E.: Structural Models of Organizational Careers: A Critical Review and New Directions, in: Breiger, R. L. (ed.): Social Mobility and Social Structure, Cambridge University Press, Cambridge $1990,272-307$

Rosenbaum, J. E.: Career Mobility in a Corporate Hierarchy, Orlando 1984

Rosenbaum, J. E.: Tournament Mobility: Career Pattems in a Corporation, in: Administrative Science Quarterly, 24, 1979a, 220-242

Rosenbaum, J. E.: Organizational Career Mobility: Promotion Chances in a Corporation during Periods of Growth and Contraction, in: American Journal of Sociology, 85, 1979b, 21-48

Ross, H.: Theorie der internen Lohnstrukar, Lang, Frankfurt 1981 
Rothschild, K. W.: Kritische Darstellung der theoretischen Grundlagen der Vollbeschäftigungspolitik, in: Vierteljahreshefte zur Wirtschufisforschung, 1, 1980, 10-51

Rowbottom, R.; Bills, D.: Organizational Design: The Work-level Approach, Gower, Cambridge 1987

Sadowski, D.: Humankapital und Organisationskapital - Zwei Grundkategorien einer ökonomischen Theorie der Personalpolitik in Unternehmen, Working Paper, Quint-Essenzen, Nr. 6, Trier 1990

Sadowski, D.; Frick B.: Unternehmerische Personalpolitik in organisationsökonomischer Perspektive: Das Beispiel der Schwerbehindertenbeschäftigung, in: Mitteilungen aus der Arbeitsmarkt und Berufsforschung, 22, 1989. 408-418

Sadowski, D.; Stengelhofen, T.: Betriebswirtschaftliche Theorie und Empirie der Nichtlohn-Arbeitskosten am Beispiel der Fluktuation der Arbeitnehmer, in: Emmerich, K.; Hardes, H. D.; Sadowski D:; Spitznagel, E. (Hsg.): Einzel- und Gesamtwirtschaftliche Aspekze des Lohnes, Beit AB 128, Nümberg 1989, 103-116

Schasse, U.: Betriebszugehörigkeitsdauer und Mobilität: Eine empirische Untersuchung zur Stabilität von Beschäftigungsverhältnissen in der Bundesrepublik Deutschland; Hannover 1990

Schein, E. H.: A Critical Look at Current Career Development Theory and Research, in: Hall, D. T. et al. (eds.): Career Development in Organizations, San Francisco 1986

Scherm, E.: Möglichkeiten und Grenzen einer untemehmerischen Arbeitsmarkfforschung, in: Zeitschrift für betriebswirtschaftliche Forschung, 10, 1991, 892-913

Schmid, G.: Der organisiente Arbeitsmarkt; Überlegungen zu einer institutionellen und politischen Theorie des Arbeitsmarktes, in: Buttler, F.; Gerlach, K.; Schmiede, R.; (Hsg.), Arbeitsmarkt und Beschäftigung, Neuere Beiträge zur institutionalistischen Arbeitsmarktanalyse, Frankfurt 1987, 24-64

Schmid, G.: Die neue institutionelle Ökonomie. Königsweg oder Holzweg zu einer Institutionentheorie des Arbeitsmarktes, in: Schmid, G.; Deutschmann, C.; Grabher, G: Die neue institutionelle Ökonomie. Kommentare aus politologischer, soziologischer und historischer Perspektive, WZB-Discussion Paper, Berlin 1988

Schnell, R.; Hill, P. B.; Esser, E.: Methoden der empirischen Sozialforschung, Oldenburg Verlag, München 1988

Scholl, R. W.: Career Lines and Employment Stability, in: Academy of Management Journal, 26, 1983, 86-103

Scholz. C.: Personalmanagement, Informationsorientierte und verhaltenstheoretische Grundlage, Vahlen, München 1989

Scholz, C.: Strategisches Management, Ein integrativer Ansatz, de Guyter, Berlin 1987

Schreuder, H.: Coase, Hayek, and Hierarchy, Research Memorandum, Rijksuniversiteit Limburg, Maastricht 1990; Als Publikation erscheinend in: Lindenberg, S. and Schreuder, H.: Interdisciplinary Perspectives on Organization Studies, Pergamon, 1993

Schreuder. H.: Economic Theories of Organization. An Overview and Assessment of Some Recent Developments, Research Memorandum 1983-11, Free University of Amsterdam, 1983

Schreuder, H.; Soeters, J.: Nationale en organisatie-culturen in accountantskantoren, in: Sociologische Gids, 33, 1986, 100-121

Schreuder, H.; Spangenberg, J.; Kunst, P.; Romme, S.: The Structure of Organizations: An Empirical Assessment of Mintzberg's Typology, Research Memorandum, Rijksuniversiteit Limburg, Maastricht 1988

Schudlich, E.: Probleme einer Theorie interner Arbeitsmärkte, in: Buttler, F.; Gerlach, K.; Schmiede R. (Hsg.): Arbeitsmarkt und Beschäfrigung, Neuere Beiträge zur institutionalistischen Arbeitsmarktanalyse, Campus, Frankfurt 1987, 158-195

Schultz-Wild, R.: Beschäftigungspolitik in der Krise, Campus, Frankfurt 1978

Schwan, R.: Theoriekonkurrenz zwischen neoklassischer und institutitioneller Arbeitsmarkttheorie: Interne Arbeitsmärkte im Fadenkreuz unterschiedlicher Theorieströmungen, Research Memorandum, Rijksuniversiteit Limburg, Maastricht 1992a

Schwan, R.: Rekrutierungsstrategien und Organisationskonfigurationen: Inwieweit sind Rekrutierungsstrategien organisationsspezifisch? Research Memorandum, Rijksuniversiteit Limburg, Maastricht 1992b

Schwan, R.: Soeters, J.; The strategy of vacancy filling from intemal and external labor market sources; An empirical assessment of the recruitment strategy of different types of organization; Research Memorandum, Rijksuniversiteit Limburg, Maastricht 1991a 
Schwan, R.; Soeters, J.: Vacancy filling and the role of organizational boundaries: A comparative empirical assessment of the influence of organizational boundaries on furms's intemal recruitment preferences, Research Memorandum, Rijksuniversiteit Limburg, Maastricht 1991b

Schwan, R.; Soeters, J.: New Insights into the Division of Organizational Labor Markets: A comparative empirical assessment of intraorganizational mobility between labor market parts (segments); Research Memorandum, Rijksuniversiteit Limburg. Maastricht $1991 \mathrm{c}$

Schwan, R.; Soeters, J.: Beschäftigungsdauer und Beschäftigungsstrukturen innerhalb betrieblicher Organisationskonfigurationen, in: Zeitschrift für Personalforschung, 6, 1992, 147-163

Scot, R. W.: Organizations: Rational, Natural and Open Systems, Prentice-Hall Inc., Englewood Cliffs, New Jersey 1987

Semlinger, K.: Vorausschauende Personalwirschaft - Betriebliche Verbreitung und infrastrukturelle Ausstattung, in: Mitteilungen aus der Arbeitsmarkt und Berufsforschung, 3, 1989, 336-347

Sengenberger, W.: Struktur und Funktionsweise von Arbeitsmärksen, Die Bundesrepublik im internationalen Vergleich, Frankfurt 1987

Sengenberger, W.: Die Gegenwärrige Arbeitslosigkeit, auch ein Strukturproblem des Arbeitsmarkts, Campus, Frankfurt 1978

Sengenberger, W.: Einführung: Die Segmentation des Arbeitsmarkts als politisches und wissenschafiliches Problem, in: Sengenberger, W. (Hsg.), Der gespaltene Arbeitsmarkt, Probleme der Arbeitsmarktsegmentation, Frankfurt 1978, $15 \mathrm{ff}$.

Sengenberger, W.: Arbeitsmarktstruktur, Ansätze zu einem Modell des segmentierten Arbeitsmarkses, München 1975

Sehringer, R.: Betriebliche Strategien der Personalrekrutierung, Ergebnisse einer Betriebsbefragung. Campus, Frankfurt 1988

Selznick, P.: TVA and the Grass Roots, A Study in the Sociology of Formal Oragnization, New York 1965

Siedenbiedel, G.: Personnelle Mohilität in Organisationen und organisierter Wandel, Eine Untersuchung potentieller Beiträge der Neuallokation von Personen und Stellen zur Organisationsentwicklung. Hannover 1984

Sievers, B.: Organisationsentwicklung und der menschliche Faktor, Zeitschrifi für Organisationsentwicklung, 7, 1988, 1-10

Singer, 0.: Lohnarbeit und Arbeilsmark, Umrisse zu einer sozial-ökonomischen Theorie der Allokation von Arbeitskrafi, Frankfurt 1986

Sitter, de U.; Hertog, den F.: Simple Organisations, Complex Jobs: The Dutch Sociotechnical Approach. Merit-Paper, Maastricht 1990

Soeters, J.: Excellent Companies as Social Movements, in: Journal of Management Studies, 3, 1986, 299 312

Soeters, J.; Keizer, P. K.: "The State of the Art": Een Tocht door de Grensgebieden in Zeven-mijls-pas, in: Keizer, P. K.; Soeters, J.: Economie, Sociologie en Psychologie, Visies op Integratie, Van Gorcum, Assen/Maastricht 1987, 15-49

Soeters, J.: Schreuder, H.: The Interaction between National and Organizational Cultures in Accounting Firms, in: Accounting, Organizations and Sociery, 13. 1988, 75-85

Soeters, J.; Schreuder, H.: Werkprestaties in Accountantskantoren, in: Maandblad voor Accountancy en Bedrifshuishoudkunde, 60, 1986b, 467-486

Soeters, J.; Schwan, R.: New Insights into the Division of Organizational Labor Markets: erscheint in: Human Relations, 1993

Soeters, J.; Schwan, R.: Towards an Empirical Assessment of Internal Labor Market Configurations, in: International Journal of Human Resources Management, 3, 1990, 271-287

Soeters, J.; Wolfs, G. L. M.: Economische Organisatietheorie en Beloningssystemen, Research Memorandum, Rijksuniversiteit Limburg, Maastricht 1991

Sorensen, A. B.: Processes of Allocation to Open and Closed Positions in Social Structure, in: Zeitschrift für Soziologie, 12, 1983, 203-224

Sorge, A.: Strategische Orientienungen des Einsatzes neuer Techniken und Arbeitsmarkt, in: Buttler, F.; Gerlach, K.; Schmiede R. (Hsg.): Arbeitsmarkt und Beschäfrigung. Neuere Beiträge zur institutionalistischen Arbeitsmarktanalyse, Campus, Frankfurt 1987, 263-290 
Sorge, A.: Informationstechnik und Arbeit im sozialen Prozeß, Arbeitsorganisation, Qualifikation und Produktivkraftenentwicklung, Campus, Frankfunt 1985

Spangenberg, J. F. A.: Economies of Atmosphere, the Joint Impact of Scale, Scope and Armosphere on Scientific Performance in Clinicul Medicine and Economics, Maastricht 1989

Spenner, K.; Otto. L. B.; Call, V. R. A.: Career Lines and Careers, Lexington Books, Lexington 1982

Spilerman. S.: Careers, Labor Market Structure and Socioeconomic Achievement, in: American Journal of Soriology, 83, 1977, 551-593

Staber. U.; Aldrich, H.: An Evolutionary View on Changes in Employment Relationships: The Evolution of Organizational Control in the United States, in: Dlugos, G. et al. (eds.), Management under Differing Labour Market and Employment Systems, De Gruyter, Berlin 1988, 63-78

Staehle. W. H.: Management, Eine verhaltenswissenschaftliche Einführung. 2. Auflage, Vahlen, München 1985

Stark, D.: Rethinking Internal Labor Markets: New Insights from a Comparative Perspective, in: American Sociological Review, 51, 1986, 492-504

Steinmann, H.; Schreyögg, G.; Thiem, J.: Strategische Personalführung - inhalttiche Ansatzpunkte und Überlegungen zu einem konzeptionellen Bezugsrahmen, in: Mitteilungen aus der Arbeitsmarkt- und Berufsforschung, 22, 1989, 397-407

Stephen, F. H. (eds.): Firms, Organization and Labour, Approaches to the Economics of Work Organization, Macmillan, London 1984

Stevens, F. C. J.: De bureaucratisering van het medisch specialistisch ambacht: een vergelijkend onderzoek naar bureaucatie en professie in twintig ziekenhuisafdelingen, Van Gorcum, Assen 1987

Stewman, S.: Demographic Models of Internal Labor Markets, in: Administrutive' Science Quarterly, 31, 1986. 212-247

Stewman, S.; Konda, S. L.: Careers and Organizational Labor Markets: Demographic Models of Organizational Behavior, in: American Journal of Sociology, 88, 1983, 637-685

Streeck, W.: The Uncertainties of Management in the Management of Uncertainty: Employers, Labor Relations and Industrial Adjustment in the 1980s, in: Österreichische Zeitschrift für Soziologie, 13, $1988,44-64$

Tabachnick, B. G.; Fidell, L. S.: Using Multivariate Statistics, 2nd. ed., Harper and Row, London 1989

Thom, N.: Personalentwicklung als Instrument der Unternehmungsführung, Stutgart 1987

Tigchelaar, L. S.: Cultuur en interne Mobiliteit, in: Management en Organisatie, 1, 1987, 22-33

Tijdens, K.: Automatisering en Vrouwenarbeid, Een Studie over Beroepensegregatie op de Arbeidsmarkt in de Administratieve Beroepen in hat Bankwezen, Uitgeverij Van Arkel, Amsterdam 1989

Tirole, J.: Hierarchies and Bureaucracies. On the Role of Collusion in Organizations, in: Journol of Low, Economics and Organization, 2,1986, 181-214

Tolbert, P. S.; Zucker, L. G.: Institutional Sources of Change in the Formal Structure of Organizations: The Diffusion of Civil Service Reform, 1880-1935, in: Administrative Science Quarterly, 28, 1983, 22-39

Tomer, J. F.: Organizational Capital, The Path to Higher Productivity and Well-being, Praeger Publishers, London 1987

Turk, J.: Conclusion: Power, Efficiency and Institutions: Some Implications of the Debate for the Scope of Economics, in: Francis, A.; Turk, J.; Willman, P. (eds.): Power, Efficiency and Institutions, A Critical Appraisal of the "Markets and Hierarchies Paradigm, Heinemann Educational Books, London 1983, 189204

Udy, S. H. Jr.: Administrative Rationality. Social Setting, and Organizational Development, in: American Journal of Sociology, 68; 1962; 299-308

Ulrich, P.: Transformation der ökonomischen Vernunft, Fortschrittsperspektiven der modernen Industriegesellschaft, Haupt, Stuttgart 1986

Vardi, Y.; Helland Hammer. T.: Intraorganizational Mobility and Career Perceptions among Rank and File Employees in Different Technologies, in: Academy of Management Journal, 20, 1977, $622-634$

Vietorisz, T.; Harrison, B.: Der arbeitsmarktendogene Ursprung von Mobilitätsbarriere, in: Sengenberger, W. (Hsg.), Der gespaltene Arbeitsmarkt, Frankfurt 1978, 99-116

Wachter, M. L.: Primary and Secondary Labor Markets: A Critique of the Dual Approach, in: Brooking Papers on Economic Activity, 3, 1974, 637-693 
Wächter, H.: Forschungsaufgaben der Personalwirtschaftslehre, in: Zeitschrift für Personalforschung. 4. $1990,55-60$

Warmerdam, J; Berg van den, J.: Nieuwe Technologieën en het Functioneren van Interne Arbeidsmarkten, Nijmegen 1986

Watts, A. G.: Career Pattems, in: Watts, A. G.; Super, D. E.; Kidd, J. M. (eds.), Career Development in Brifain, Hobsons Press, Cambridge 1981, 213-245

Weber, M.: Wirlschaft und Gesellschaft, Tübingen 1972 und 1976

White, H. C.: Chains of Opportunity: System Models of Mobility in Organizations, Harvard University Press, Cambridge, Mass. 1970

White, R. W.; Althauser, R. P.: Internal Labor Markets, Promotions, and Worker Skill: An Indirect Test of Skill LMs, in: Social Science Research, 13, 1984, 373-392

Wholey, D. R.: Deterninants of Firm Intemal Labor Markets in Large Law Firms, in: Administrative Science Quarterly, 30, 1985, 318-335

Wilber, C. K.; Harrison R. S.: The Methodological Basis of Institutional Economics: Pattern Model, Storytelling, and Holism, in: Journal of Economic Issues, XUI, 1978, 61-89

Williamson, 0. E.: Comparative Economic Organization: The Analysis of Discrete Structural Altematives, in: Administrative Science Quarterly. 36, 1991, 269-296

Williamson, O. E.: Reflections on the New Institutional Economics, in: Zeitschrift für die gesamte Staatswissenschaft, 141, 1985, 187-195

Williamson, O. E.: Efficient Labour Organization, in: Stephen F. H. (ed.), Firms, Organizations and Labour; Approaches to the Economics of Work Organization, Macmillan, London 1984

Williamson, O. E.: The Economics of Govemance: Framework and Implications, in: Zeitschrift für die gesamse Staatswissenschaft, 140, 1984, 195-223

Williamson, O. E.: The Economics of Organization: The Transaction Cost Approach, in: American Journal of Sociology, 87, 1981, 548-577

Williamson, O.E.; Transaction-Cost Economics: the Governance of Contractual Relations, in: The Journal of Law and Economics, 1979, 233-261

Williamson, O. E.: Markets and Hierarchies, Free Press, London/New York 1975

Williamson, O. E.; Ouchi, W. G.: The Markets and Hierarchies Programme of Research: Origins, Implications, Prospects, in: Francis, A.; Turk, J.; Willman, P. (eds.): Power, Efficiency and Institutions, a Critical Appraisal of the Markets and Hierarchies Paradigm, Heinemann Educational Books, London 1983, 13-34

Willianson, O. E.; Wachter, M. L.; Harris, J. E.: Understanding the Employment Relation: The Analysis of Idiosyncratic Exchange, in: Bell Journal of Economics, 6, 1975, 250-278

Willman, P.: The Organisational Framework and Industrial Sociology, in: Francis, A.; Turk, J.; Willman, P. (eds.): Power, Efficiency and Institutions, a Critical Appraisal of the Markets and Hierarchies Paradigm, Heinemann Educational Books, London 1983, 117-136

Windmuller, J. P.; Galan, C.; de; Zweeden, A. F. van: Arbeidsverhoudingen in Nederland, Aula. Utrecht 1987

Windolf, P.; Hohn, H. W.: Arheitsmarkschancen in der Krise, betriebliche Rekrutierung und soziale Schließung, Campus, Frankfurt 1984

Windolf, P; Wood, S.: Recruitment and Selection in the Labour Market: A Comparitive Srudy of Britain and West Germany, Gower, 1988

Wöhe, G.: Einführung in die Allgemeine Betriebswirtschaftslehre, 11. Auflage, München 1973

Wolfs, G. L. M.: Firm Internal Labour Markets in the Netherlands, A Contract-Theoretical Approach, Maastricht 1992

Wolfs, G. L. M.: Tenure in the Dutch Economy, Research Memorandum, Rijksuniversiteit Limburg. Maastricht 1990

Wolfs, G. L. M.: Pricing and Allocation on Intemal Labour Markets, Research Memorandum, Rijksuniversiteit Limburg, Maastricht 1988

Woodward, J.: Industrial Organization: Theory and Practice, London 1965

Wunderer, R.: Betriebswirtschaftslehre und Führung - Entwicklungslinien, Besonderheiten, Funktionen, in: ders. (Hsg.), Betriebswirtschafislehre als Management- und F ührungslehre, Poeschel, Stuttgart 1985. 237-267 
Wunderer, R.: Betriebswirtschaftslehre als Management- und Führungslehre, Poeschel Verlag, Stuttgart 1985

Yin, R. K.: Case Study Research, Sage, London 1985

Zucker, L. G. (ed.): Institutional Patterns and Organizations, Culture and Environment, Ballinger Publishing Company, Cambridge, Massachusetts 1988

Zucker, L. G.: Institutional Theories of Organization, in: American Sociological Review , 13, 1987, 433-446 


\section{Curriculum vitae}

Rolf Schwan ist geboren in Wuppertal. Nach der Nichtschülerreifeprüfung studierte er Wirtschaftswissenschaften an der Gesamthochschule Wuppertal mit den Schwerpunkten Betriebswirtschaftslehre, Industriesoziologie, Volkswirtschaftslehre sowie Personalwirtschaft und Organisationslehre. Das Studium wurde durch Arbeitsaufenthalte in Kanada, USA und Japan ergänzt.

Nach dem Abschluß als Diplom-Ökonom war er im elterlichen Betrieb als Geschäftsführer für die Personalbetreuung verantwortlich. Seinen Berufsweg setzte er fort als Personalberater bei Nixdorf-Computer.

Als Projektleiter im VDI-Technologiezentrum Informationstechnik Berlin koordinierte er im Auftrag des Ministeriums für Forschung und Technologie das internationale Projekt zur Akzeptanz von Informationstechnologie im Büro- und Produktionsbereich. Im Rahmen der Projektberatung wurden organisatorische und personalentwicklungsbezogene Vorschläge erarbeitet und umgesetzt.

An der Rijksuniversiteit Limburg in Maastricht/Niederlande wurde er mit der Leitung des Forschungsprojektes 'interner Arbeitsmarkt' beauftragt. Produkt dieser Forschungs- und Lehrtätigkeit (Betriebswirtschaftslehre, Organisations- und Personalmanagement $u$. a.) ist die vorliegende Dissertation. Ein Teil der Forschungstätigkeit erfolgte als Mitarbeiter des 'Institute For Research On Intercultural Cooperation' (IRIC) unter Leitung von Prof. Dr. G. Hofstede.

Rolf Schwan arbeitet als FachhochschulJehrer an der Fachhochschule in Aachen. 


\section{Organisationskonfigurationen und interne Arbeitsmărkte \\ Rolf Schwan}

Inteme Arbeitsmârkte bilden den Krouzungapunkt aus Arbeitsmarkt- und Organisationstheorie. Aus personalwirtschattlicher Sicht ruckt eine der wichtigstan Formen betrieblicher Bescheltigung in den Mittelpunkt. Zwei sich ergãrzende Forschurgs: strómungen charakterisieren die Optuk, aus der die Arbelt entstanden ist.

Eine Forschungsstromung dleset Untersuchung besteht aus der noch jungen Forschungstradition der 'neuen Strukturalisten', Damil sind jene Vertreter eines nevon Paradigmas angesprochen, die den EinfluB struktureller Faktoren auf die beruliche Mobilltat betonen. Dieser Ansatz ist ais der Krlik an Statuszuweisungs: und Humankapitalansatz entstanden. Whihrend beide Ansatzs mehr die Angebolsselie des: Arbeitsmarkles hervorheben, akzentuieren die neuen Strukturalisten mehr die Nachfrageseite (Artbeilgeber) bzw. struktumtle Faktoren; wie z. B. der Einfluß won Organisationsmeriknalen.

Als andere Forschungisströmung ist die betriabsbezogene Arbeilsmarkt- und Benulsforschung anz. sprechen. Hiler verbinden sich bel der Ekkenntris. suche Elemente der Organisations- und Berulssozologie, angereichert durch eine beinebsorientierte Mikrolundiemg der Allokation van Arbeit.

Adressatenkreis der Pesultale dleser Untersuchung sind Fuhrungakrăfte der Personal und Organisationsabtellungen. Untemehmensberater sowia interossiente Forschet. 